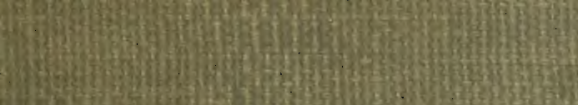
(5)

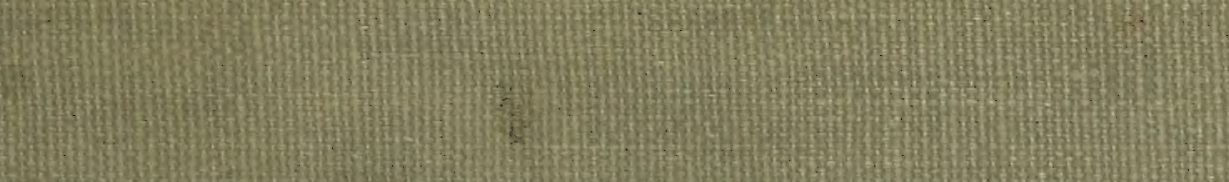

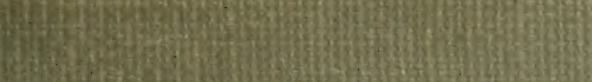

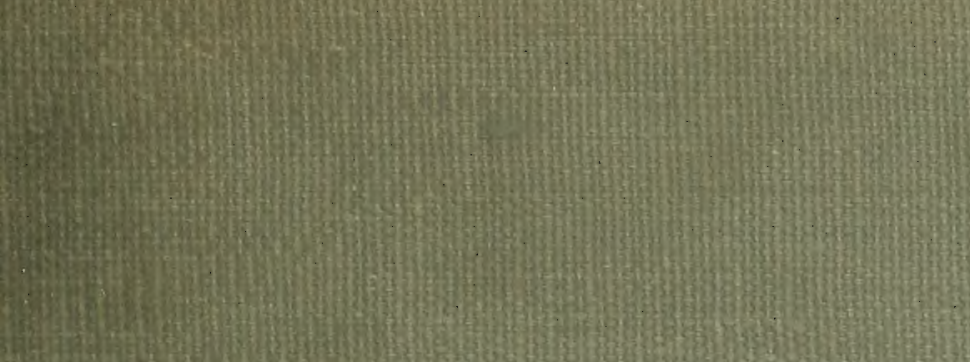
(1) ait

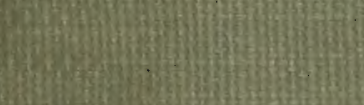

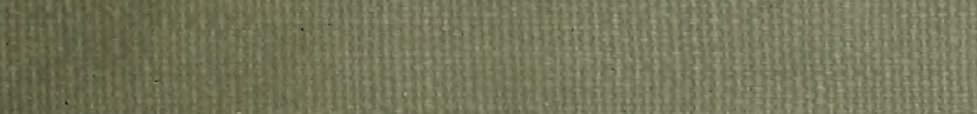
(s)

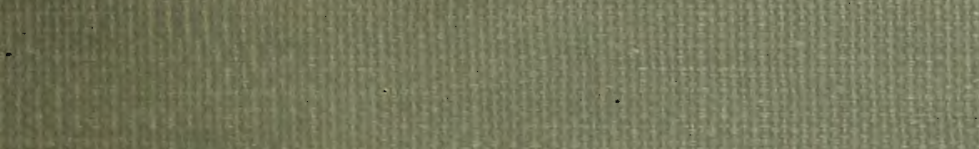
$-\frac{10}{20}$

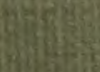

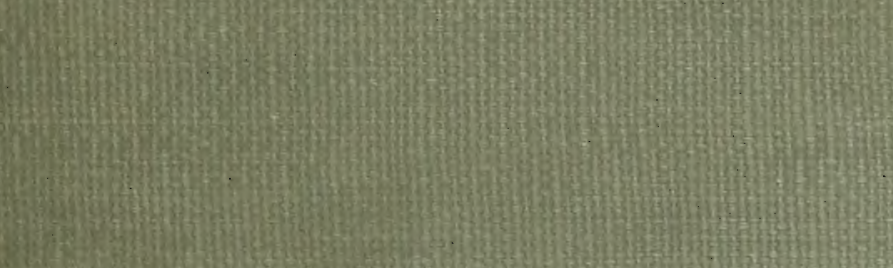

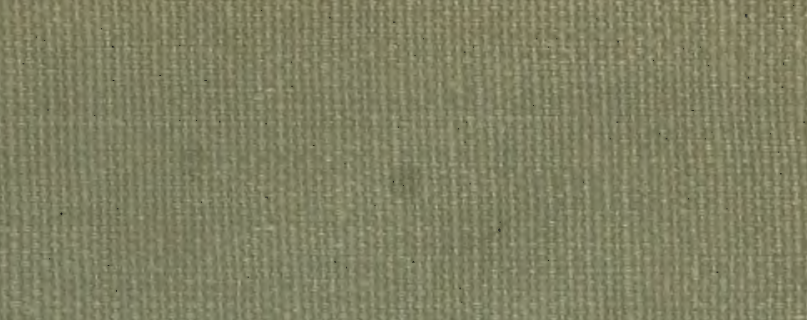
(1)

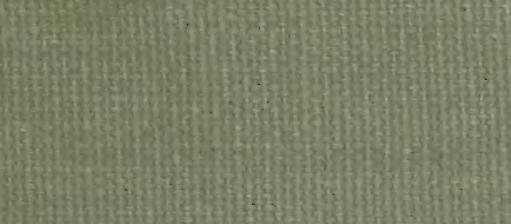



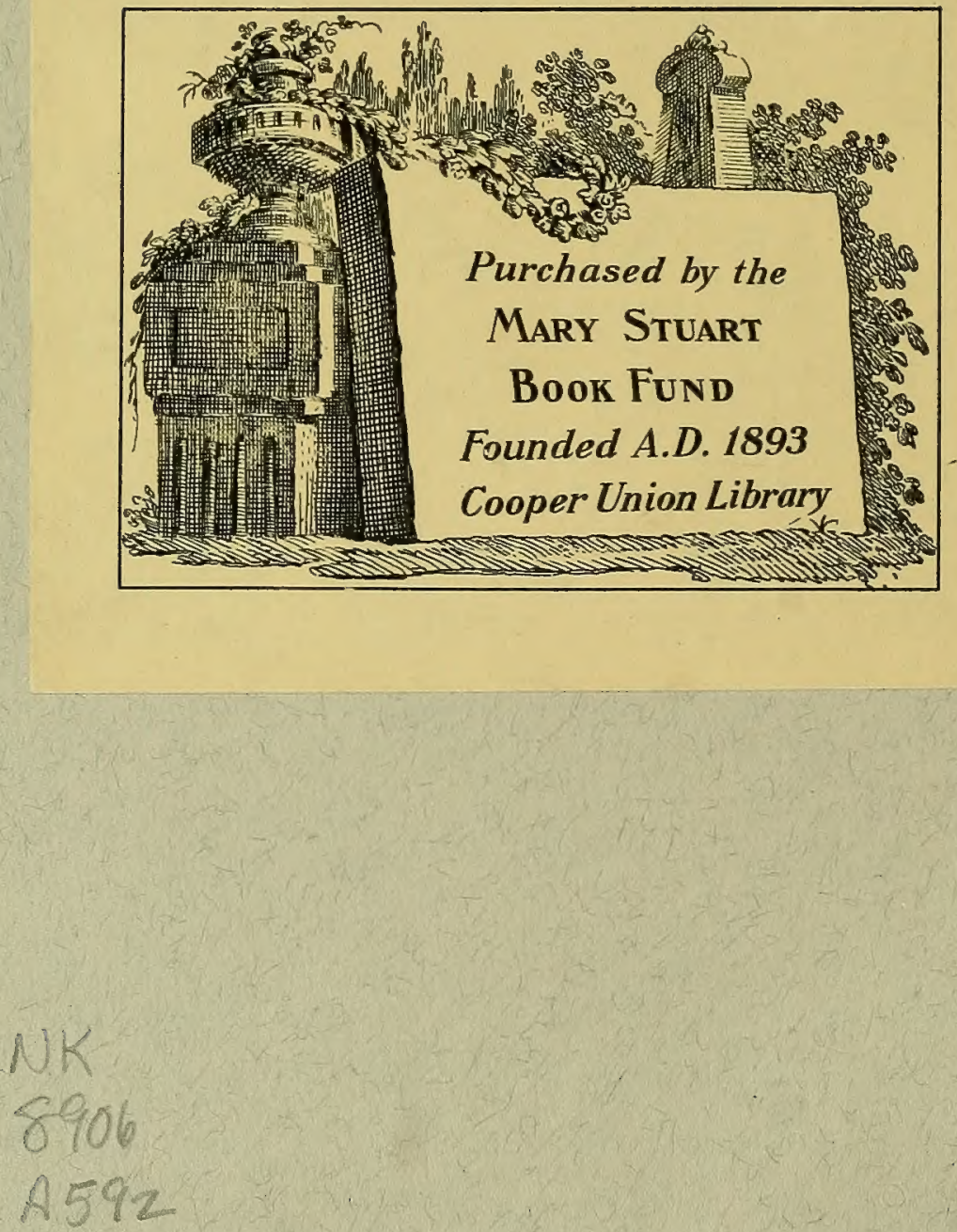






GR A M M A IR E

DES

ARTS DE LA SOIE 
DU MEME AUTEUR:

\title{
GASPARD GRÉGOIRE ET SES VELOURS D'ART
}

1 vol. in-8 sur vélin d'Arches, avec 7 phototypies hors texle

$$
\text { PRIX : } 5 \text { FRANCS }
$$

Société Française d'Imprimerie et de Librairie, Ancienne librairie Lecène OUdN \& $C^{\text {ic}}$, Paris, 1908.

\author{
EN VENTE A LA LIBRAIRIE JEAN SCHEMIT :
}

\section{LA FLEUR DE LA SCIENCE DE POURTRAICTURE}

PATRONS DE BRODERIE FAÇON ARABICQUE ET YTALIQUE

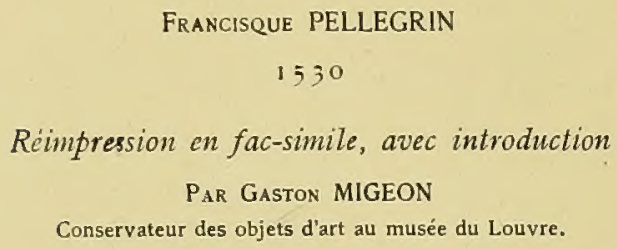




\title{
GRA II M A IRE
}

\author{
DE S
}

\section{ARTS DE LA SOIE}

PAR

\section{Henri A LGOUU}

Ouvrage illustré de qualre-vingt-six gravures

\author{
PAR IS \\ J E A N S C H E M I T \\ LIBRAIRE DE LA SOCIÉTÉ DE L'HISTOIRE DE L'ART FIANGGAIS \\ 52, RUE LAFFITTE, 52 \\ 1912
}

Tous droits de traduction et de reproduction réservés pour lous pays. 


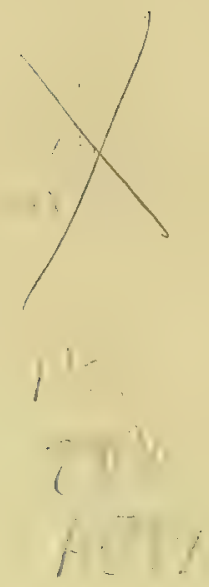

MAR 221950

309719 


\section{GR A M M A I R E}

\section{Des \\ A RTS DE LA SOIE}

\section{AVA NT-PROPOS}

Sole, Soleries, sont des mots qui reviennent à chaque instant, si l'on $y$ prend garde, dans le langage usuel et qui affirment l'importance du rôle joué tous les jours par les réalités auxquelles ils correspondent: le plus recberché, le plus précieux des textiles et tout ce que l'industrie bumaine en a su faire depuis qu'elle le connaît.

L'étude de la soie et des étoffes faites de soie est par suite, on se le figure, un vaste champ qui n'est certes point resté en friche; mais il est à remarquer que ce sujet très étendu semble avoir été exploité jusqu'ici, surtout à deux points de vue particuliers et à peu près exclusifs: l'bistoire de la soie et des tissus de soie dans le sens arcbéologique et documentaire et, d'autre part, la technique pure de la production de la soie et de la fabrication des soieries.

Cependant, à côté des nombreux travaux historiques d'une savante érudition et des traités théoriques et spéciaux sur le tissage, n'y avait-il pas de place pour un ouvrage, peut-être plus abordable, c'est-à-dire simplifié et généralisé dans un but de vulgarisation?

C'est la pensée qui a inspiré cette Grammaire, sorte d'essai résumé sur les choses nombreuses, variées et curieuses de la Soie et des Arts de la Soie.

Le tissu de soie n'est plus comme jadis l'apanage d'une classe assez restreinte, surtout privilégiée de la naissance ou de la fortune : on peut dire à juste titre qu'il s'est aujourd'bui largement démocratisé dans ses applications et ses emplois très divers, parfois même assez imprévus.

Il occupe une part prépondérante dans l'babillement fëminin, au principal ou pour partie; qu'il s'agisse de la mondaine réputée pour la recherche de coûteuses élégances, de la petite bourgeoise plus discrètement satisfaite, ou simplement de l'ouvrière qui aime à se parer du bout de ruban ou du 
chiffon de mousseline; il se maintient bonorablement pour l'accessoire dans le costume masculin et va du gilet, de la cravate et du chapeau du clubman, en passant par la robe du professeur et du juge, au foulard et à la casquette de l'bomme du peuple avec l'accompagnement de l'universel et prudbommesque parapluie!

Pour l'ornementation, l'ameublement des intérieurs cossus, aussi bien que des palais, on ne peut se passer de la richesse décorative unique du tissu de soie, qui flotte encore au vent arec nos drapeaux et monte à la conquête de l'air avec les ballons!

Et combien d'autres emplois dits de fantaisie, très justement: garnitures de toutes sortes, fanfreluches, accessoires, fleurs artificielles, objets de luxe divers, lui demandent cette note d'élégance, même mesurée au poids de la soie, qu'il faut à mille riens ingénieusement combinés, artistement chiffonnés.

Or, puisque le tissu de soie est ainsi partout maintenant, ne pouvait-il être intéressant d'essaver de faire généralement mieux concevoir les détails de sa fabrication? Fabrication aux origines lointaines, au passé glorieux, devenue la très grande industrie qui chaque jour prend, dans le monde entier, un plus large développement.

La tâche était pour cela de chercher à mettre à la portée d'un peu tout le monde, en évitant le plus possible les termes de métier, un ensemble de notions empruntées à l'art du tissage proprement dit, notions succinctes et condensées, mais suffisamment précises pour être opposées à des obscurités et des inexactitudes assę répandues en pareille matière.

Il importait encore, au cours de comparaisons tout indiquées entre les vieilles soies d'autrefois et les jeunes chiffons d'aujourd'bui, de rappeler quelques noms de ceux, invenieurs, artisans, artistes et fabricants babiles, dont l'bistoire de la soie peut à bon droit s'enorgueillir, parmi la masse de tant d'autres efforts restés anonymes, et confondus; enfin, de mettre en valeur, tout en la résumant, la combinaison remarquable du talent du dessinateur avec l'babile expérience du tisseur, alliance féconde qui est tout l'Art de la Décoration du tissu.

Et maintenant, c'est au lecteur de décider si nous avons réussi dans une entreprise qui n'a pas visé plus baut que de retenir son attention avec quelque utilité, peut-être. 


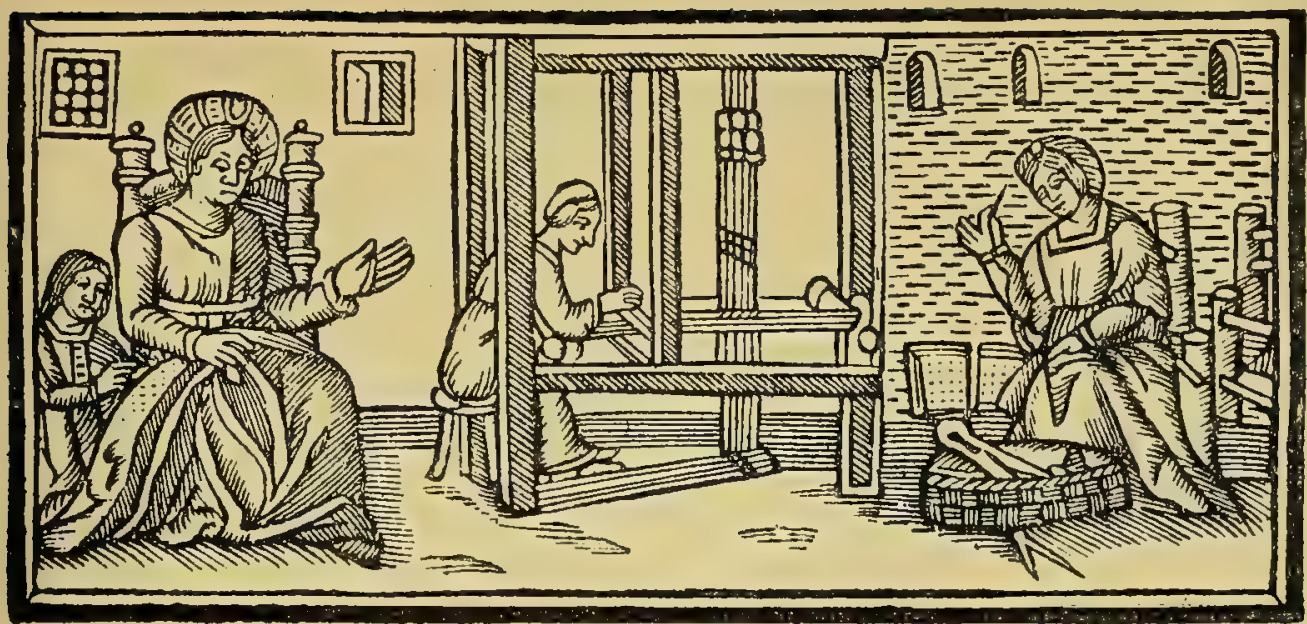

TISSAGE ET BRODERIE (d'après le Livre de lingerie, 1584).

\section{QUELQUES MOTS D'HISTOIRE}

\section{VIEILLES SOIES - JEUNES CHIFFONS}

Les qualités qui valent à la Soie d'occuper le premier rang parmi les textiles sont trop connues, trop évidentes pour que leur démonstration s'impose.

Depuis que ce fil ténu, souple, incomparablement résistant pour son extrême finesse, est connu de l'humanité, on voit qu'elle a apprécié hautement les caractères typiques des tissus ingénieusement fabriqués avec cette matière. Brillant sans pareil, aspect chatoyant et riche, solidité, légèreté, toucher inimitable, en un mot, tout ce que le langage a résumé, condensé dans la signification de l'épithète "Soyeux» qui dit précisément tout cet ensemble. Et le langage ne s'est pas borné non plus à emprunter au tissage, né de la nécessité du vètement, nombre de métaphores et d'aphorismes en altérant parfois le sens juste des mots, mais il a coloré plusieurs de ces expressions d'attributs qui sont comme la preuve de l'excellence, de la prééminence du précieux fil.

«Des jours filés d'Or et de Soie », cela ne se dit plus guère évidemment qui en poésie, et la tournure a vieilli, mais Satins, Velours, Gazes et autres soieries évocatrices de magnificences, ou plus simplement d'élégances raffinées, prètent encore à des effets goûtés en littérature.

D'autres Beaux-Arts ont rendu un hommage éclatant à la Soie, aux Soies $^{1}$. Il suffit de considérer tout l'important, l'heureux parti tiré par la

1 Nous prenons ici le mot dans une acception littéralement inexacte sans doute: celle d'étoffes elles-mêmes, la partie prise pour le tout; l'erreur est si généralement commise et 
peinture, utilisant une richesse décorative unique, des oppositions nuancées, des coloris flatteurs, par l'imitation habile des étoffes soyeuses, et leur copie, souvent très fidèle, est précieuse à titre de documents.

Mais, de même que pour la Dentelle dont bien des points se retrouvent fixés exactement et savamment par le pinceau ou le crayon, ce serait toute une étude à faire que de rechercher d'examiner de près le rôle joué par la soie, et qu'elle continue à jouer plus sobrement peut-être de nos jours, dans de si nombreuses œuvres célèbres, scènes religieuses allégoriques, historiques, portraits surtout : et les tableaux des Primitifs où sont reproduits à la manière plate et presque calquée les brocarts et les velours rehaussés d'or, les toiles où les peintres de la Renaissance firent briller somptueusement les Damas, les Lampas, les chefs-d'œurre des maîtres portraitistes de toutes les écoles et de tous les temps ', où furent imités, prodigués avec talent et avec charme les superbes reflets, les plis seyants et harmonieux des belles Soies, sont comme autant de places d'honneur et de titres de gloire pour un fil unissant à l'excellence la richesse et la rareté qui distingue.

Rare, la soie le fut si bien, qu'aux époques reculées, nous dit-on, les pontifes et les dames romaines, avides de se parer de vêtements faits avec des soieries, apportées à grands frais de contrées mystérieusement lointaines, les payaient leur propre poids d'or. Au début du Moyen Age, les étoffes de soie étaient encore mises sur le même rang pour les échanges que les métaux précieux; elles figuraient parmi les objets que devaient fournir les peuples soumis à payer un tribut, ou encore occupaient une place très importante dans les présents que se faisaient les uns aux autres de puissants souverains; témoin celles qui furent envoyées à Charlemagne par Haroun-Al-Raschid et plus tard à l'empereur Frédéric Barberousse par un chef musulman. Longtemps les Draps de Soie et les Draps de Soie mêlés dOr, magnifiques produits des ateliers orientaux, restèrent recherchés à l'égal des choses les plus estimées. Il est tout naturel dès lors de les voir affectés à la première heure aux usages sacrés, composant les ornements du culte dans les églises, enveloppant les reliquaires et les châsses. C'est ainsi qu'à l'ombre des cathédrales et des abbayes se conservèrent jusqu'à nos jours d'inestimables documents tissés, ayant heureusement résisté aux atteintes destructives du temps, plus funestes encore, on le conçoit, aux tissus qu'à toutes choses.

D'autres, ensevelis pendant des siècles sous le sable qui cachait les sépultures, comme à Antinoé, devaient revoir la lumière, tels qu'ils avaient orné tuniques et manteaux d'une société à demi romaine et à demi chrétienne, et nous faire connaître quels habiles tisseurs furent les Coptes. Mais ces premières considérations nous entraînent un peu forcément à envisager dans

devenue usuelle, peut-être à cause de l'euphonie meilleure et de la brièveté plus grande, que nous n'avons pas hésité à faire à l'usage cette concession.

1 Nombreux sont les peintres qui ont excellé dans la reproduction des étoffes de soie. La liste en serait longue, à l'établir consciencieusement. Indiquer des noms comme ceux de Titien, Véronèse, Van Dyck, Largillière, Nattier, La Tour, c'est tout au plus amorcer une recherche qu'il est aisé pour chacun de poursuivre dans les musées et d'étendre à maints ouvrages. On a cité, par exemple, les satins de Terburg et de ses imitateurs; il ne faudrait pas non plus négliger Ingres qui a fait des satins et des velours étonnants de vérité, etc. 
un très rapide aperçu, nécessairement incomplet, ce que furent les origines lointaines de la soie, et aussi le chemin qu'elle a parcouru dans le monde et à travers les années accumulées avant d'en arriver à ses usages si communément répandus aujourd'hui. On voudra bien, puisque notre cadre nous impose d'être brefs en ceci, se reporter pour plus de détails aux nombreux ouvrages consacrés à ce sujet.

Les historiens nous apprennent que de temps immémorial les peuples de 1a Perse, de la Chine et de l'Inde, pays des Sères, ou province Sérique - il faut voir, paraît-il, dans ces noms Sères, Sérique, l'étymologie du mot Soie - connurent le ver se nourrissant de la feuille du mûrier et produisant le cocon dont ils savaient utiliser la matière pour en tisser des étoffes. Il s'écoula un temps impossible à apprécier autrement qu'en siècles, avant que les peuples occidentaux eussent connaissance de ces étoffes et du textile qui les composait. Aucune affirmation très certaine n'étant possible ni permise à cet égard, il est fort probable que les étoffes de soie furent apportées d'abord d'A sie en Europe par des voyageurs a ventureux, fort avant que s'opérât la

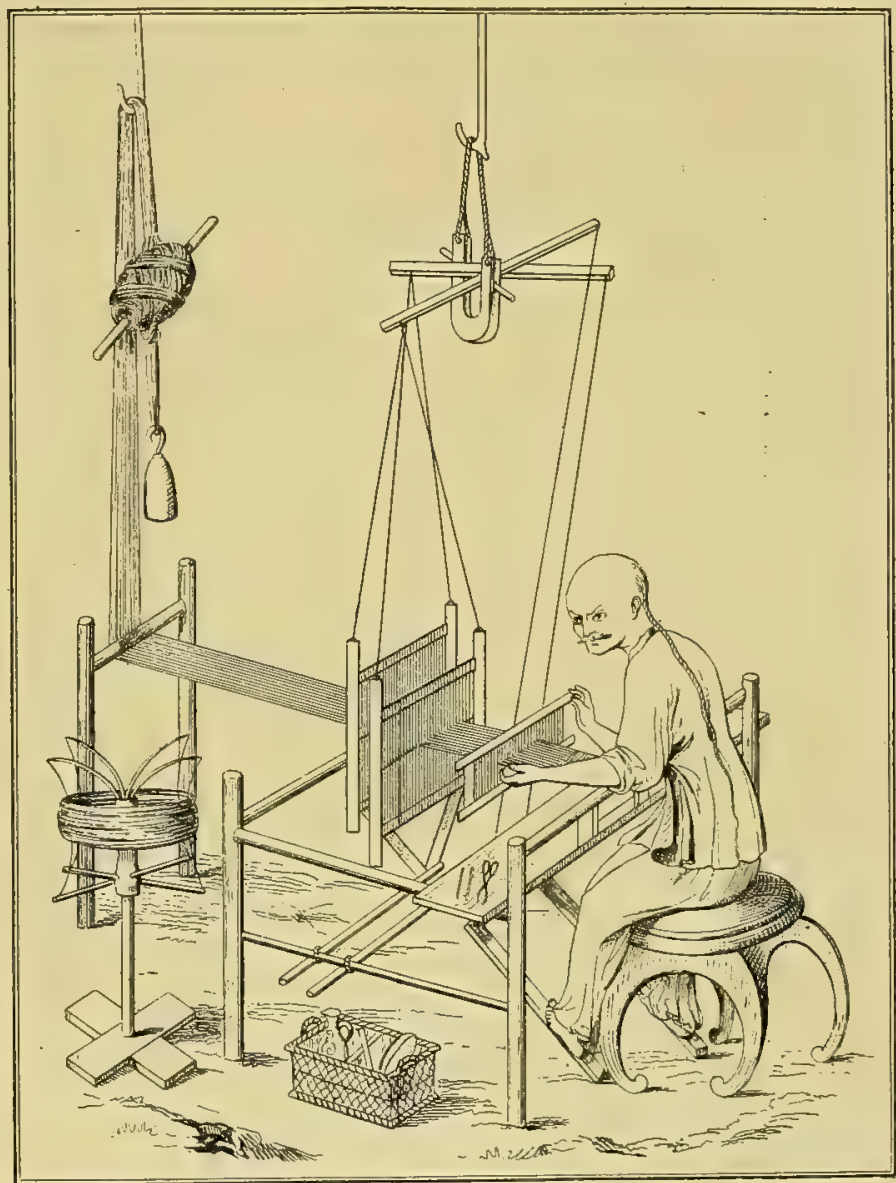

Métier chinols (d'après Falcot).

transmission de la manière d'obtenir la Soie elle-même avec les moyens indispensables : les œufs de l'animal dont se trouve ainsi utilisée remarquablement une période de la vie, et le mûrier portant la feuille dont il se nourrit exclusivement à l'état de chenille.

La légende attribue tantôt à une princesse asiatique, tantôt à des moines ou pèlerins explorateurs le mérite de ce transport à Byzance, sous Justinien, après que fut surpris le secret jalousement gardé par les peuples détenteurs de l'ingénieux procédé. 
Les écrivains s'accordent cependant à dire que le fait eut lieu vers le milieu du $\mathrm{VI}^{\mathrm{c}}$ siècle de notre ère, et qu'à cette époque seulement, la soie vint se mêler à la laine, au lin', au coton, employés jusqu'alors, ce que paraissent démontrer les fragments précieux dus à l'art tout spécial des Coptes, exhumés lors des fouilles faites à Antinoé.

Ils disent encore, ces auteurs, que les premières manufactures de soieries européennes furent établies dans les principales villes de la Grèce, région dont le climat convenait fort bien à la culture du mûrier. Presque en même temps, l'heureuse découverte était exploitée par la civilisation arabe et propagée par elle sur les côtes d'A frique et jusqu'en Espagne, où les soieries de Grenade, de Séville, de Valence et d'Alméria furent longtemps célèbres et recherchées.

Nous avons mentionné la valeur attachée aux œuvres d'habiles ouvriers, à une époque où la plus grande partie de l'Europe sortait à peine de la barbarie. Un grand nombre de ces artisans de la soie trouvaient un abri hospitalier dans le Royaume des Deux-Siciles, où, vers i I 30 , le roi Roger les attira en favorisant leur établissement à Palerme et dans la Calabre, conséquence sans doute des goûts de luxe pris durant les Croisades au contact d'infidèles souvent fastueux, malgré les défenses du Coran. Les ateliers palermitains devaient atteindre à une renommée que fait comprendre la beauté de quelques-unes de leurs productions, conservées jusqu'à nos jours:

De la Sicile, la culture du mûrier, la soie et les artisans de la soie se répandirent peu à peu dans toute l'Italie. Il appartint longtemps à Venise; que sa toute-puissance maritime rendait maîtresse du commerce de la Méditerranée, de détenir le monopole du trafic des riches étoffes de soie venues des lieux de production orientaux, à l'usage de ses nobles auxquels seuls ce luxe était permis par les lois de la République et des nations étrangères, ses clientes: Non contente de cette importation, la riche cité fabriqua bientôt elle-même des soieries; Venise eut ses manufactures, comme plusieurs autres villes de la Péninsule: Florence, Gênes, qui donna son nom à un type de velours qui porte encore cette appellation, Sienne, où l'on tissait et brodait à peu près uniquement des ornements d'églises, Lucques, qui eut aussi cette spécialité, parmi tant d'autres, et acquit la réputation consacrée par la locution française : "A Lucques sont les bonnes Soyes. »

Car la France, qui demanda souvent à 1a brillante Société de la Renaissance italienne les enseignements de ses incomparables artistes, fut longtemps aussi un débouché considérable et des plus profitables pour ses fabriques et ses marchands de soieries.

Rien d'étonnant par suite à ce qu'on eût bientôt en France l'idée d'y établir une fabrication qui prospérait de cette façon de l'autre côté des Alpes.

Elle avait commencé déjà quelque peu dans le Comtat-Venaissin avec l'introduction du Mûrier et du Ver à soie, à la fin du XiII ${ }^{\circ}$ siècle, par des compatriotes du Dante. La résidence des Papes à Avignon fut certainement un grand encouragement momentané à l'industrie naissante, qui ne devait pas néanmoins progresser dans la région jusqu'à un très grand développement sous le rapport fabrication, mais qui marqua toutefois le début de l'installation de la Sériciculture et du tissage de la Soie dans une grande partie de la 
Provence. Les filatures y restent nombreuses, surtout dans les Cévennes, si les métiers se sont raréfiés à Nîmes, où il y en eut bon nombre à certaine époque, et ne sont plus que simple souvenir, à Aix-en-Provence, par exemple.

Le premier des actes officiels, par lesquels la Royauté montra l'intérêt qu'elle prenait à l'organisation en France de la richesse commerciale due à la fabrication des soieries, fut des lettres patentes accordées par Louis XI, en I 466, pour l'établissement d'une manufacture à Lyon, sous certaines conditions fiscales, qui, détail piquant, soulevèrent d'abord les protestations des lyonnais contre la faveur qu'on leur accordait. La priorité pour cette ville ne peut ainsi faire de doute, contrairement à une tradition généralement admise que la première manufacture d'étoffes de soie établie en France le fut à Tours, en 1470 , encore avec la protection et la sollicitude de Louis XI, qui avait fait venir et avait installé dans sa ville de prédilection divers artisans d'Italie, «tous ouvriers et faiseurs de draps de Soye ». En réalité, la fabrique lyonnaise n'avait pas progressé beaucoup en ces quatre années; l'importation des étoffes italiennes continuait à se faire largement. Mais plus tard, les lettres patentes en forme de charte, données par François ${ }^{\text {er }}$ en 1536 , à Lyon, à son retour de Savoie, et les avantages et privilèges ainsi concédés aux ouvriers étrangers, les attirèrent en cette ville, déclarée en même temps l'unique entrepôt des soies et des soieries qui entraient en France et par lequel toutes devaient passer.

Deux Génois, Etienne Turqueti et Barthélemy Nariz, appelés, dit-on, par M. de Gadagne, descendant d'une famille Gadagni, émigrée à Lyon 'd'Italie depuis assez longtemps, furent les premiers à bénéficier de ces dispositions accueillantes et à fonder une manufacture destinée à prospérer; ils se joignirent au nombre déjà élevé de leurs compatriotes établis dans l'ancienne Lugdunum, tant pour y exercer leurs talents professionnels que pour y faire fructueusement la banque et le commerce.

Dans les années qui suivirent, le goût des Français ne se démentit nullement, pour les belles et coûteuses étoffes de soie, et celles où l'or et l'argent entraient souvent pour partie si importante. La fameuse entrevue du Camp du Drap d'Or, oú, selon l'expression d'un chroniqueur, « plusieurs y portèrent leurs moulins, leurs forêts et leurs prez sur leurs épaules », fut comme le signe de l'énorme consommation de ces riches tissus qui se fit au $\mathrm{XVI}^{\mathrm{e}}$ siècle et que les fabriques de France étaient alors incapables d'alimenter à elles seules, troublées qu'elles furent de plus par les guerres de Religion. Le tribut payé de ce fait à limportation étrangère, italienne surtout, restait considérable.

Deux hommes, sous Henri IV, eurent le mérite d'efforts tentés avec persistance pour diminuer cette redevance onéreuse et pour développer la production intérieure: Olivier de Serres, avec ses écrits sur la Cueillette de la Soye, et surtout Laffemas, que son génie actif, entreprenant, conduisit à exposer la situation au roi en véritable économiste averti, dans des mémoires pressants où il le poussait à prendre toutes mesures, à rendre tous édits pour favoriser l'essor de ce qu'il entrevoyait devoir être une des gloires et des richesses nationales.

Henri IV, bien digne de comprendre ces représentations, ne marchanda pas la protection requise; par ses ordres, on s'occupa de maintenir, de 
restaurer et d'étendre la plantation des mûriers partout où elle avait été entreprise et jusque dans les Résidences royales. Il y eut même, rapporte-t-on, un curieux essai fait à Paris : 20.000 pieds de cet arbre furent mis dans le Jardin des Tuileries et on y éleva des vers à soie dans l'orangerie. Les manufactures existantes furent encouragées, multipliées. On créa à Paris, au logis de la Macque, faisant partie de l'ancien Hôtel d'Anjou, rue de la Tixeranderie, des ateliers où l'on fabriquait des étoffes de luxe. En 1602, des ouvriers français, qu'on y avait appelés, faisaient à Paris les satins façon de Gênes et aussi toute espèce d'autres étoffes, d'Italie ou d'ailleurs, « si bien, dit Laffemas dans un rapport, que la Majesté du Roy en a été satisfaite, comme plusieurs princes et seigneurs qui y étaient présents ». Enfin, en I604, on construisit au Parc Royal des Tournelles un vaste bâtiment destiné à recevoir des manufactures, où les métiers fonctionnèrent en 1606 , et les « entrepreneurs » de ces métiers avaient été anoblis.

Ces tentatives ne furent pas, malheureusement, couronnées du succès qu'on en attendait; au commencement du Xvir siècle, seules les manufactures de Lyon et de Tours, sans compter celles du Midi de la France, restaient pour lutter contre l'invasion des soieries étrangères; leurs produits, sans doute, valaient bien celles-ci. Le Cardinal de Richelieu le dit positivement, et cependant, défenses, édits, prohibitions passagères, du reste, etc., n'arrivaient pas à détourner le penchant de nos ancêtres à rechercher ce qui venait de loin, soigneusement entretenu par les marchands, qui ne se faisaient pas faute, en outre, d'user de ruses habiles pour tromper sur la qualité comme sur la provenance de leurs étoffes.

Du moins la voie avait été tracée; le ministre éclairé de Louis XIV la suivit avec l'attention vigilante qu'il portait à tout ce qui était susceptible d'augmenter la prospérité du royaume. Colbert, en supprimant les barrières des douanes intérieures de province à province et ne laissant subsister que le seul bureau de douane de Lyon pour toutes les soies, avec l'institution de droits protecteurs sur celles provenant de l'étranger, montra l'intérêt qu'il portait à la manufacture de cette ville; en outre, il s'occupa d'y réglementer, malgré des résistances assez vives, le bon fonctionnement corporatif et les rapports entre Maitres-ouvriers, Ouvriers et Maîtres-marchands. Sous cette impulsion régulatrice et énergique, la fabrication et le commerce lyonnais des étoffes de soie connurent des années de réelle prospérité.

De cette époque date le commencement de la renommée qui devait rendre Lyon célèbre dans le monde entier et lui donner bientôt la suprématie sur les autres fabriques par la perfection de ses soieries et le talent de ses dessinateurs; ceux-ci, formés à l'école de Lebrun, le décorateur fameux, officiel, du Grand Siècle, surent, de ce moment, s'affranchir des formules décoratives trop répétées en Italie, et créer un véritable style, avec d'ingénieuses améliorations d'exécution de leurs dessins.

Dès lors, bien que l'industrieuse cité dût éprouver cependant encore des secousses profondes, venues de causes politiques, comme la révocation de l'Édit de Nantes, en i685, qui chassa quantité de ses meilleurs artisans vers des contrées étrangères, où ils apportèrent, pour certaines, une industrie nouvelle, et traverser des moments troublés par les dissensions intérieures, on peut dire que l'intérêt historique se concentre sur sa fabrique. 
On la voit ainsi primer celle d'Avignon, décimée par la peste de I722, oú le nombre des métiers décroît pendant tout le XvIII ${ }^{e}$ siècle, pour tomber à rien de nos jours; celle de Nîmes, qui se cantonna assez vite dans la bonneterie de soie; celle de Tours, qui n'arriva pas à maintenir le rang qu'elle avait occupé un moment et, en diminuant progressivement d'importance au point de vue nombre de métiers, devait seulement garder la spécialité de beaux tissus d'ameublement.

En nommant Paris, où le travail de la soie n'atteignit pas un développement permettant de classer la Capitale au nombre des réels centres manufacturiers de cet ordre, il serait injuste de ne pas parler de la création sous Louis XIV, en 1677 , à Saint-Maur-les-Fossés, d'une manufacture d'étoffes de Soie par le célèbre Marcelin Charlier, « homme d'une intelligence toute particulière pour faire fabriquer toutes sortes d'étoffes $\gg$, disait-on de lui alors, et qui, fournisseur du Grand Roi et de sa Cour, fit sortir de ses ateliers de riches tissus d'ameublement, parmi lesquels de magnifiques velours, qui ajoutèrent encore à sa réputation, en même temps qu'ils excitaient l'admiration générale.

Les règnes de Louis XV et de Louis XVI virent le plein épanouissement de la fabrication lyonnaise, accentuant une supériorité désormais incontestable sur toutes ses rivales. Ce n'est pas trop s'avancer en disant que, nulle part et jamais mieux, on ne sut y travailler la soie et dans tous les genres. En effet, ses fabricants, aidés de la collaboration inappréciable de dessinateurs qui furent, pour la plupart, grands par le mérite de la composition adaptée à l'exécution et par le goût parfait, produisirent souvent de véritables chefs-d'œuvre, qui ont constamment depuis servi de modèles; mais, à côté d'œuvres aussi relevées et artistiques, ils surent faire plus modestement des étoffes simples, de prix plus accessibles, ce qu'on appelle aujourd'hui le courant, avec beaucoup d'habileté, de soin et même de recherche.

Tous les noms seraient presque à citer parmi la pléiade d'hommes remarquables, associant leurs efforts pour l'œuvre qui est la gloire de Lyon, s'occupant à perfectionner incessamment les moyens du tissage, comme à augmenter encore la beauté et la richesse des étoffes; mais un seul les domine tous : celui du décorateur le plus merveilleux, en même temps que créateur connaissant toutes les ressources, toutes les délicatesses et toutes les forces d'un art plein de difficultés et de subtiles connaissances, Philippe de Lasalle, que, par un juste hommage rendu à son talent inégalé, on a nommé parfois le Raphaël de la soie.

Les événements de la Révolution devaient malheureusement arrêter ce magnifique essor, et le siège de Lyon marqua la ruine de son industrie, à tel point qu'on put la redouter complète; mais les bases en étaient trop profondes et les traditions trop bien implantées, pour disparaître en quelques années désastreuses; Napoléon, premier consul et empereur, voulant faire revivre son activité, trouva dans cette ville de Lyon, pour laquelle il montra un intérèt soutenu, des tisseurs toujours expérimentés, des artistes qui surent façonner leur talent au style nouveau et rivaliser entre eux pour la décoration somptueuse des palais impériaux, et aussi des inventeurs comme Jacquard.

Le relèvement de l'industrie lyonnaise fut rapide et durable. Attribuer, d'ailleurs, à l'invention de la célèbre mécanique de Jacquard, le point de 
départ de lorientation sans cesse grandissante que devait prendre au $\mathrm{XIX}^{c}$ siècle la fabrication des soieries à Lyon, serait exagéré; incontestablement, la mise en pratique de cet appareil, apportant des facilités inconnues jusqualors pour l'exécution des tissus à dessins, marque une date considérable au point de vue textile. Mais, sans vouloir diminuer à beaucoup près les mérites de 1'homme et de son œuvre, trop méconnus en leur temps, et sur

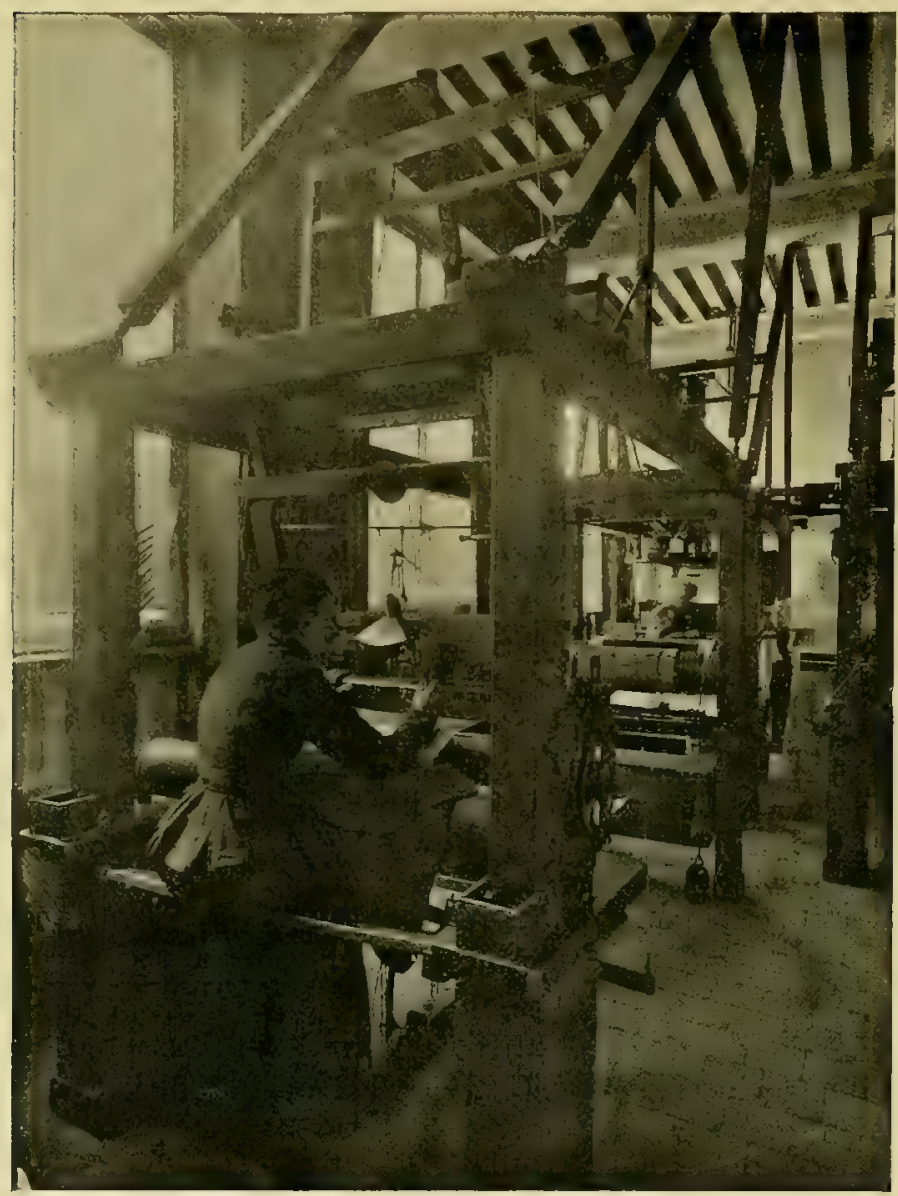

Atelier DE tissage A BRAS DE SOIERIES, A Lyon. lesquels nous reviendrons, il nous paraît juste de voir à cette évolution et même à cette transformation si complète des causes multiples, économiques et sociales.

L'abolition, à partir des années révolutionnaires, du système corporatif, qui restreignait forcément la production en la limitant, fut une modification profonde du travail à Lyon et, désormais, les étoffes de soie trouvèrent acheteurs dans une classe singulièrement élargie sous l'influence des mêmes idées, avec nécessité de s'adapter, sous le rapport du coût, à des emplois devenant de plus en plus communs, non seulement en France, mais chez les nations qu'à son tour elle eut à fournir, et de plus en plus largement, de ces produits de luxe recherchés pour leur supériorité; car les fabriques d'Espagne n'étaient plus guère que souvenirs, celles d'Allemagne et de Suisse ne pouvaient entrer en ligne de compte que pour des genres assez étroitement limités, celles d'Italie et de Russie s'organisaient ou se réorganisaient; l'Angleterre, suivant les idées de Cobden, allait renoncer à soutenir les siennes, et 1'A mérique s'ouvrait comme un pays neuf et inexploité : toutes conditions qui furent de puissants stimulants pour le développement considérable que l'on relève alors. L'abondance de la matière première venue des contrées de climat favorable au mûrier et 
à l'élève du ver - Provence, Piẻmont, Sicile, Asie Mineure - allait s'augmenter des soies d'abord grossières, mais bientôt améliorées, apportées de l'Extrême-Orient, Indes, Chine et Japon.

Aussi, vers la moitié du $\mathrm{xix}^{\mathrm{e}}$ siècle, il y avait près de 100.000 métiers tissant la soie à Lyon: métiers à bras, battant, selon l'expression consacrée, pour fabriquer Taffetas, Failles, Velours, Damas, Lampas, etc., et faisant entendre incessamment le bruit familièrement représenté par des onomatopées comme celles bien connues des Lyonnais : Bistien claque pan! et Patintaque! à la Croix-Rousse, à Saint-Georges, Saint-Irénée et autres quartiers habités par les canuts d'alors. C'était le temps des ateliers de

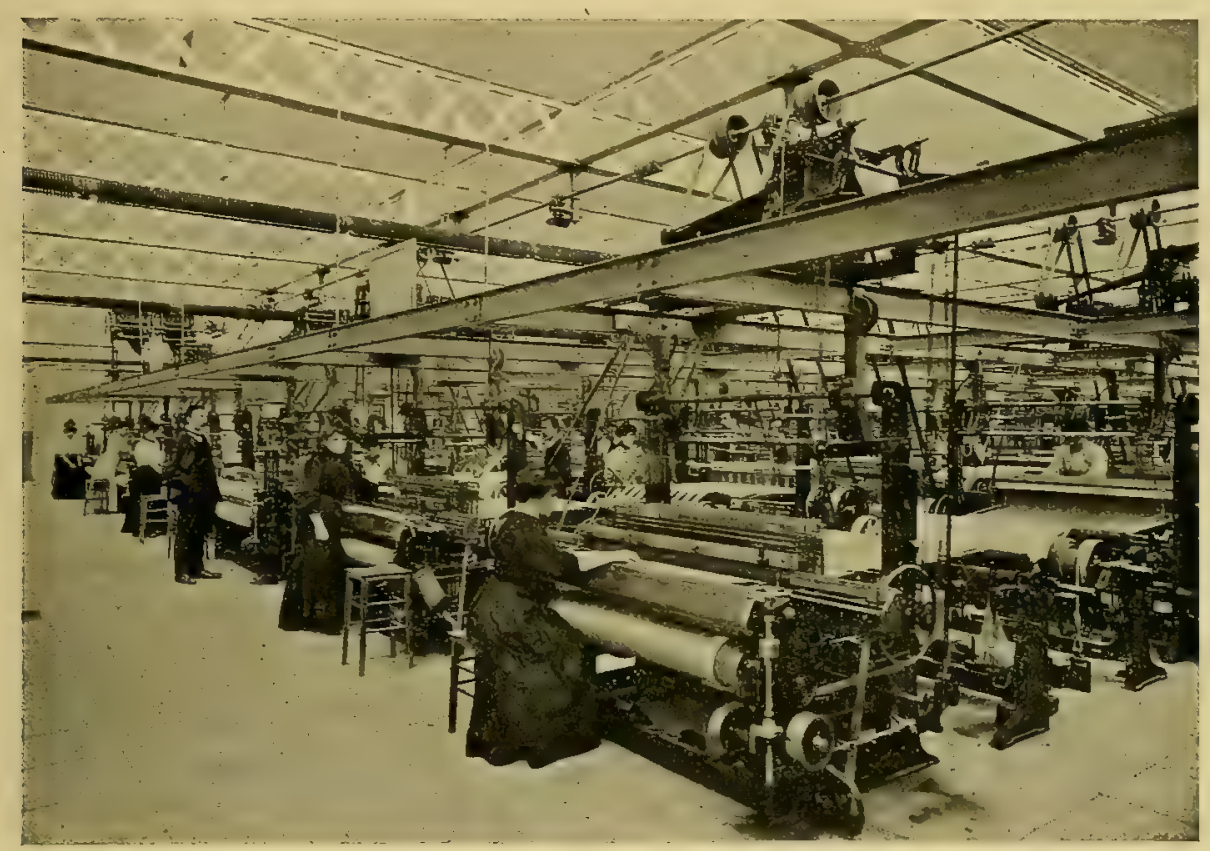

Atelier de tissage mécanioue de soleries ${ }^{1 .}$

famille si typiques, comprenant un petit nombre de métiers; là, depuis le chef de la famille, le Maître canut, aidé pour le tissage d'ourriers et d'ouvrières dits compagnons et compagnonnes, jusqu'à sa femme et ses enfants occupés aux besognes accessoires et de préparation, tous exerçaient pour la commune tâche le tissage à façon, pour le compte des patrons fabricants, une admirable habileté manuelle et professionnelle développée par l'atavisme et l'habitude et qui est restée proverbiale. C'était " l'Airt (pour l'Art) de la Soie », comme le qualifiaient les canuts eux-mêmes en leur parler aux expressions curieusement spéciales, entremêlées de termes empruntés au rocabulaire du tissage soyeux et pleines de saveur pour qui les a un peu pratiquées. La naïveté patoise de ce langage, que le Guignol lyonnais a popularisé quelque

1 M. Henry Bertrand, Lyon. 
peu, cachait une bonhomie malicieuse et ironique, car le canut fut volontiers frondeur et parfois émeutier; ses ancêtres avaient renvoyé avec des chansons satiriques l'illustre Vaucanson venu pour réglementer leur travail; lui, fit le coup de feu en $183_{4}$ et en I 848 .

Mais de grands changements ne tardèrent pas à survenir. L'application du moteur mécanique au métier et la transformation, par suite, radicale de cet instrument séculaire dans son principe de mise en action, vinrent naturellement, on peut le dire, en un siècle de vapeur et d'électricité, pour répondre aux besoins d'une production qu'il fallait augmenter et de prix à diminuer au contraire. Le problème fut bientôt résolu pour la soie comme il l'avait été pour le coton, par exemple, et dans tous les pays où l'on fabriquait des tissus de soie et oủ l'on ne chercha, dès lors, qu'à en faire plus et à meilleur marché. Ce travail en usine s'organisa au détriment de celui à la main, relégué hors des villes, dans les campagnes, oú son salaire devint souvent un appoint seulement à la vie matérielle assurée en partie déjà. Aujourd'hui, il n'y a plus ou presque plus de ces canuts de jadis, sauf pour les quelques rares étoffes dont la difficulté d'exécution réclame leurs doigts habiles à manier les fils légers, à les faire se croiser, s'entremêler patiemment sans défauts, selon les règles, et s'accommode de la lenteur forcée du travail à la main.

Doit-on regretter cette transformation et l'éloignement pour les belles étoffes, richement décorées, sous l'empire de la mode qui les a délaissées longtemps ? C'est un peu l'avis de ceux qu'enchante la vue des vieilles Soies, empruntant, il est vrai, une partie de leur charme à leur âge même, mais évoquant de façon souveraine, dans les musées, les collections, ou quand elles sortent des tiroirs et des resserres pour d'intéressantes Expositions, l'art étonnant des décorateurs et des fabricants qui les créèrent et les exécutèrent souvent sans les moyens perfectionnés d'à présent.

Faut-il dire avec d'autres, les partisans déterminés du « tout passe, tout se transforme », qu'il n'y a plus guère de place pour les belles choses qui nécessitent trop de temps et trop de soins minutieux; et encore, que la mécanique a permis de mettre à la portée du plus grand nombre une élégance à bon marché, répandue plus heureusement dans les mille choses du vêtement, par exemple, que mesurée à prix d'or dans quelques tentures somptueuses. Enfin, ces défenseurs du moderne progrès font remarquer, non sansjustesse, que, de perfectionnement en perfectionnement, on en est arrivé, avec le métier méeanique, à tisser avec une régularité surprenante, que ne pouvaient souvent pas donner les moyens manuels, des Gazes, des Mousselines, des Velours, et toute la catégorie des tissus extra-souples, «flous, » en un mot, tout ce qui a fait la vogue du «Chiffon » du $\mathrm{xx}^{e}$ siècle; et cela, dans des proportions si grandes qu'elles déterminent une activité commerciale se chiffrant par centaines de millions de francs, rien que pour Lyon, et maintiennent du travail à des milliers d'ouvriers, dans plusieurs départements français.

Ce serait bien impossible de trancher entre les deux opinions ou de les concilier en quelque sorte. En attendant ce que sera pour l'avenir de la soie en Europe un lendemain impossible à prévoir de façon certaine, et, peut-être, tributaire lui-même à son tour de l'industrie poussée à la façon ultra-moderne des pays nouveaux, Amérique ou Japon, on peut, au moins, émettre un vœu 
platonique. Il faut bien, obligatoirement, suivre la marche imprimée à la fabrication des soieries par la domination inéluctable de la Mode, et les entraînements du luxe tout-puissant et envahissant qu'on ne saurait, en réalité, condamner; sa cessation serait un mal et même une calamité pour quantité d'industries, et La Fontaine a dit trop justement :

\section{La République a bien affaire}

De gens qui ne dépensent rien :

Je ne sais d'homme nécessaire,

Que celui dont le luxe épand beaucoup de bien,

Nous en usons, Dieu sait! Notre plaisir occupe

L'artisan, le vendeur, celui qui fait la jupe

Et celle qui la porte...

Mais, tout en se conformant à l'influence des mours, des idées, des goûts qui évoluent, on devrait ne jamais oublier tout à fait le passé dans ses enseignements. Et non seulement ceci pour rendre un tribut d'admiration mérité à tant d'efforts patients, habiles, qui se sont accumulés les uns avec les autres dans cet exode de la soie qui l'a faite mondiale à travers les époques et se sont condensés souvent en modèles de purs styles, mais pour conserver de ceux-ci utilement, certains diraient pieusement, et tout au moins dans la mesure du possible, la direction impeccable au point de vue composition, exécution et valeur artistique.

La faute en est évidemment à l'arrangement hâtif de la vie d'à présent, si l'on ne peut plus considérer avec le respect et les égards d'autrefois les soieries diverses largement répandues maintenant, sur lesquelles les yeux s'arrêtent encore avec complaisance, mais que les mains manient parfois sans trop de ménagements. On ne réfléchit guère, faute de temps, que le tissu, surtout le tissu de soie, est obtenu par une sorte de longue synthèse, pour parler à la façon chimique, unissant des éléments simples en un tout assemblé par de multiples opérations et, en l'espéce, par les manipulations successives. Le moindre ordinaire Taffetas, «fond de Jupe » ou «petite Soie » a cependant passé par une vingtaine de " mains » principales et par bien d'autres, si l'on tient compte de toutes les manipulations accessoires. C'est une vaste collaboration qui s'établit dès l'élève du ver à soie jusqu'au placard ou rayon du marchand, et s'accompagne de transactions fréquentes, de transports réitérés; marche lente ou rapide, coupée d'arrêts indispensables et représentant au total une complexité, un détail, qui valent que l'on s'y arrête pas à pas, en essayant de donner de ces nombreuses étapes de la fabrication une idée plus exacte que celle que l'on en conçoit généralement. 


\section{COMMENT S'OBTIENT LA SOIE}

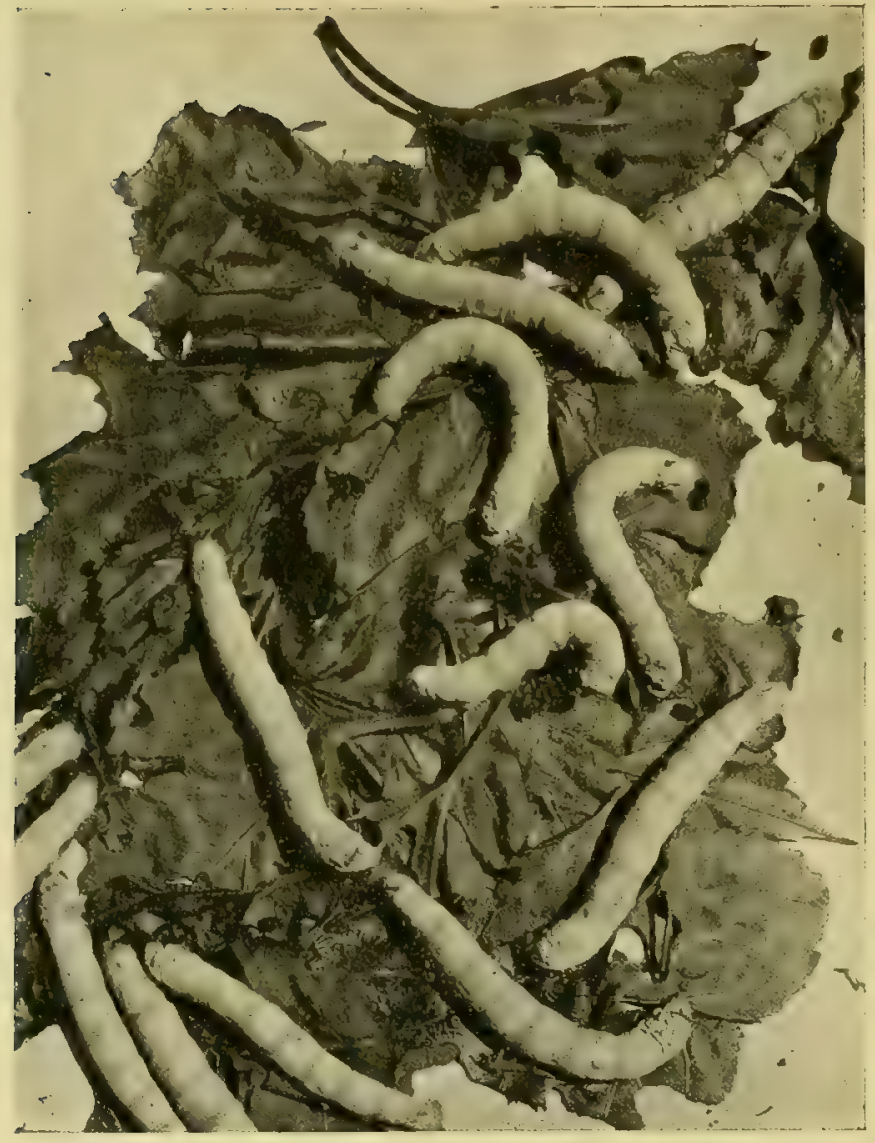

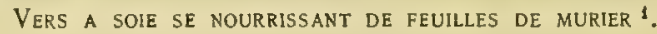

La soie est le fil dont s'enveloppe 1a chenille du mûrier a u nom savant de Bombyx Mori ou Phalcena Bombyx pour se métamorphoser en chrysalide. Cette enveloppe est le Cocon, sorte de coque cylindrique aux bouts arrondis et légèrement étranglée en son centre; elle est entièrement formée du fil replié quantité de fois sur lui-même en spires agglutinées, à la façon d'une petite pelote creuse.

La matière soyeuse existe chez le ver à soie, à l'exemple de nombreuses autres chenilles et araignées, sous la forme d'un liquide visqueux, sécrété par un organe spécial, se coagulant à l'air en fils qui servent à assurer la suspension de l'animal ou un abri pour ses transformations.

Dans les contrées suffisamment chaudes, comme certaines parties de la Chine, où le ver à soie peut vivre sur l'arbre, son éducation et la récolte du cocon se sont faites longtemps à l'air libre. Cet élevage, dans nos régions, est forcément l'objet de soins plus méthodiques en chambres closes, qui constituent la science de la sériciculture. L'élève du ver à soie, qui fut souvent dans nos campagnes un revenu accessoire des travaux des champs, pratiquée

1 Cliché du Laboratoire d'études de la Condition des Soies de Lyon. 
dans une des pièces de la maison familiale, a lieu maintenant surtout dans des bâtiments spéciaux dits magnaneries, du nom populaire magnan, donné dans le midi de la France à la précieuse chenille; il s'agit de profiter des quelques semaines où l'insecte vit à l'état de larve. C'est

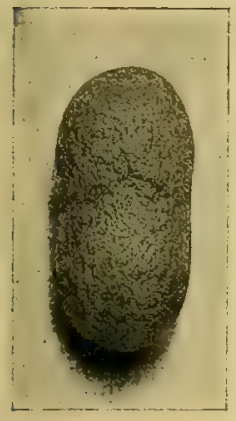

COCON FILÉ vers la mi-avril qu'on met à l'éclosion, après une sélection dont Pasteur a montré l'utilité, les œufs minuscules ou graines, comme on les appelle, dont il faut 1.300 environ pour faire le poids de I gramme. Maintenus dans les conditions reconnues nécessaires de chaleur constante et de ventilation, ils donnent bientôt naissance à de très petites chenilles, longues à peine de 2 millimètres; celles-ci, placées dans le même milieu favorable sur des sortes de rayonnages ou claies; grossissent rapidement; on leur fournit en abondance la feuille du mûrier qui est leur nourriture exclusive et quili faut ni trop sèche ni trop humide. Au bout de trente jours environ et après avoir subi plusieurs mues ou changements PAR LE VER A SOIE ${ }^{1}$. de peau, les vers à sole ont atteint tout leur développement, soit 8 à ro centimètres de longueur et un poids de 4 á 5 grammes. C'est à ce moment qu'ils montent sur des petits rameaux de bruyère ou de bouleaux qu'on a déposés en éventail sur les claies et s'y. installent pour filer leurs cocons, travail qui prend trois ou quatre jours environ. Enfermés ainsi dans leur coque soyeuse, ils se transforment aussitôt en chrysalides et, une vingtaine de jours plus tard, en papillons, accomplissant ainsi le cycle fixé par la nature à l'espèce.

Comme ce papillon, pour sortir du cocon, le perce et le rend par là mème très difficilement utilisable, ne sont conservés tels, une fois filés, que les cocons dont on a besoin pour assurer la reproduction par l'accouplement des papillons mâles et femelles; ceux-ci pondent la précieuse graine à conserver avec des précautions parmi lesquelles se place l'action indispensable d'une température assez basse, et même froide, jusqu'à la prochaine éclosion qui a lieu, en général, l'année suivante, mais il y a certaines races, dites polyvoltines, qui ont plusieurs générations annuellement, comme il $\mathrm{y}$ a du reste nombre d'autres sortes de chenilles vivant à l'état sauvage sur divers arbres, et produisant un cocon dont certains trouvent une utilisation importante, par exemple ceux donnant la soie dite

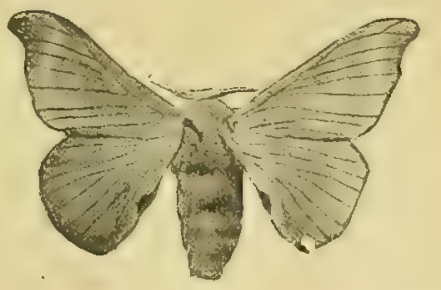

Pafillons de vers a sole 1 .

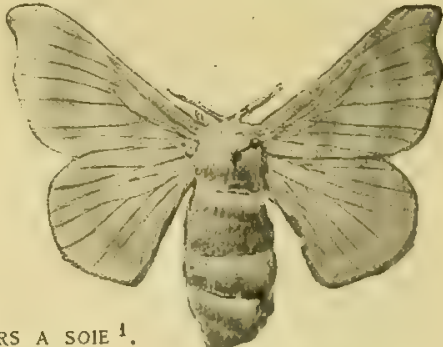

Tussah ou Tussor.

La chaleur est le meilleur moyen d'étouffer les chrysalides pour prévenir leur métamorphose à l'intérieur des cocons et afin de pouvoir conserver ceux-ci autant qu'il est nécessaire. Primitivement, on se contentait donc d'exposer la

1 Cliché du Laboratoire d'études de la Condition des Soies de Lyon. 
masse des cocons récoltés aux forts rayons du soleil; actuellement, un four convenablement disposé et chauffé est un moyen plus expéditif et plus sûr.

Comment tirer de cette petite chose sèche, légère, d'apparence diversement colorée, en jaune pâle, jaune d'or, blanc jaunâtre ou tirant sur le vert, selon la race et le pays du ver qui l'a construit, le beau fil que l'on connaît pour son brillant et sa souplesse. C'est d'abord par la filature, opérée maintenant dans les organisations en usines de ce nom. Filer la soie, c'est dérouler, dévider ce fil replié, comme nous l'avons dit, sur lui-même et collé à luimême. Il est nécessaire, pour cela, de maintenir les cocons plongés dans l'eau très chaude, presque bouillante d'une bassine de cuivre; bientôt leur enveloppe est suffisamment ramollie pour que l'ouvrière fileuse puisse, en les battant légèrement avec un petit balai de bruyère, faire s'accrocher aux aspérités de cet instrument simple et pratique le bout extérieur du fil. Toute une première partie, commencement du travail du ver, est grossière, rugueuse, irrégulière; elle est rejetée et forme les déchets, nommés frisons, pour lesquels on a trouvé d'ailleurs une utilisation.

La fileuse arrive, de la sorte, à disposer d'un certain nombre de cocons dont le fil est, comme on dit, purgé et se dévide aisément. On pourrait croire qu'il n'y a plus qu'à tirer sur ce fil et à l'enrouler, mais, d'une part, ce fil, ou plutôt ce brin pour parler précisément, n est pas de longueur indéfinie et mesure, certains disent, 300 , d'autres jusqu'à 800 mètres au plus, sauf exceptions, mais encore il est bien trop ténu, trop fin pour être employable tel qu'il est; de plus, il serait irrégulier, car son diamètre va constamment en décroissant, jusqu'à devenir presque imperceptible.

Pour assurer la régularité, la continuité et la grosseur convenable au fil à produire, l'habileté de la fileuse consiste, avec une dextérité acquise par la pratique, à assembler en un seul les brins de deux, quatre, six ou huit cocons, selon les données dune expérience qui fait prévoir le résultat; ces brins, ramollis par l'humidité et la chaleur, se collent les uns aux autres pour former un brin unique; après s'être croisé pendant quelques tours avec un brin voisin formé de la même manière, ceci pour que leur frottement réciproque les arrondisse, celui-ci va s'enrouler sur un tambour dont le mouvement l'entraîne et sur lequel il se sèche. La fileuse doit encore veiller avec soin et voir quand il faut, par un adroit tour de main, jeter le bout, c'està-dire ajouter un nouveau brin d'un cocon prêt à être filé et tenu en réserve sur le bord de la bassine, pour remplacer le brin d'un cocon finissant ou trop ténu. C'est au jugé, avec une approximation remarquable due à l'entraînement des yeux et des doigts exercés, qu'elle arrive à maintenir ainsi la régularité de grosseur du fil, autrement dit son titre, première des conditions réclamées par les emplois industriels d'aujourd'hui.

La soie ainsi filée se présente sous l'aspect d'un fil écru, résistant, coloré comme l'était le cocon, peu brillant, et de toucher assez sec et prend le nom de Soie Grège; on en forme de grands écheveaux ou flottes, tordues sur elles-mêmes, et de ces flottes on compose des ballots ou balles, dont le transport, l'achat, la vente, la revente, le courtage forment autant de branches commerciales actives et importantes sur les grands marchés de la soie. Trains et navires apportent par wagons et chargements entiers ce fret précieux, dont l'abondance ou la rareté fait le prix, crée le cours ou la 


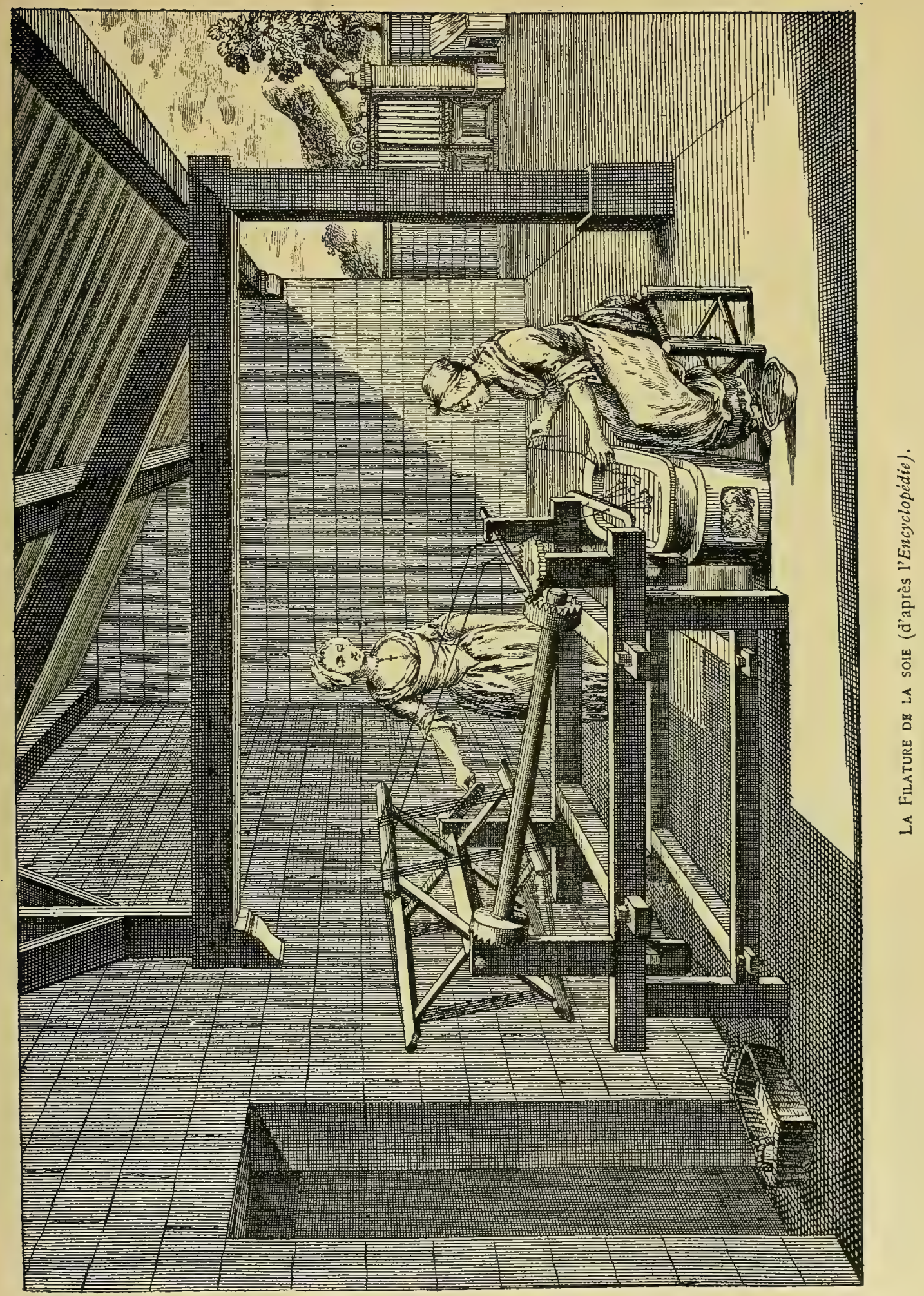


cote, divisée en infinités de classes par genres, provenances, catégories et marques ${ }^{1}$.

Là, on titre la soie et on l'essaye ; c'est-à-dire qu'on vérifie sa grosseur, sa résistance et toutes qualités annoncées par la filature, utiles pour son classement et l'établissement de sa valeur marchande. Et aussi, on la conditionne; en effet, la soie, qui a des propriétés électriques bien connues, est

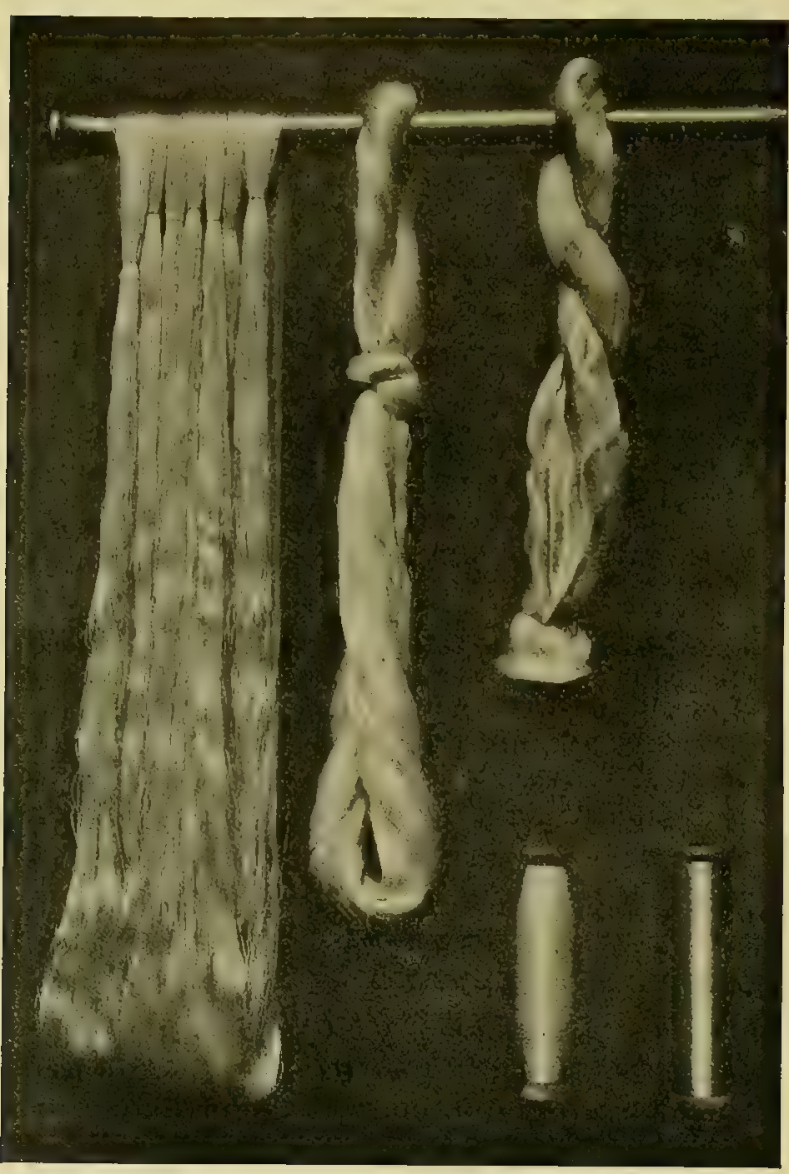

Flottes et Bobines de SOle. aussi très hygrométrique; or, comme elle se vend au poids, il importe d'établir pratiquement celui qui servira de base au calcul de la somme due par l'acheteur au vendeur. C'est ce qui a lieu dans des établissements fonctionnant officiellement sur les grandes places comme Lyon, Milan, Zurich, etc. Le procédé consiste à prendre un certain nombre de flottes de la balle soumise à l'opération et à leur faire subir dans des appareils à ce destinés une absolue dessiccation. I1 s'ensuit une perte de poids que l'on note très exactement, et un calcul proportionnel fournit ensuite, en tenant compte d'un degrẻ d'humidité fixé par les usages à I I pour Ioo d'eau, le poids total cherché, qu'on porte sur un bulletin destiné à faire foi dans la transaction.

Voilà donc le fil de soie entre les mains des fabricants, prêt à être mis sur le métier et à être transformé en étoffe, pourrait-on penser? C'est inexact cependant de façon générale. Il existe bien des tissus faits entièrement de soie grège et employés ainsi

1 Il serait intéressant de pouvoir retrouver quel a été le prix de la soie en remontant á travers les âges; nous avons dit qu'à l'époque romaine elle s'échangeait contre environ son poids d'or. Ainsi, par des calculs de comparaison, les auteurs ont pu montrer que, sous Justinien, la valeur du kilogramme de soie ou plus exactement de soierie était de 17 . Igo francs environ de notre monnaie. En I345, la soie de Provence coûtait 76 sous tournois la livre, ce qui équivaut de nos jours à 65 francs. La moyenne des prix actuels de la soie écrue s'établit de façon approchée à 40 francs le kilogramme. 
en écru; mais la très grande majorité des autres, qu'ils soient teints en fils ou tissés teints, c'est-à-dire composés de fils teints au préalable avant le tissage, ou teints en pièces, c'est-à-dire fabriqués avec des fils écrus et teints ensuite, exigent pour leur confection que ces fils aient subi une torsion préparatoire calculée. C'est l'opération du moulinage ou de l'ouvraison. Au moyen d'appareils nommés moulins, on tord les fils sur eux-mêmes, en les assemblant parfois par deux, trois, etc., pour n'en faire qu'un et en graduant ces torsions, ces assemblages d'après le genre d'étoffes à fabriquer et le rôle qu'auraient à jouer ces fils dans cette étoffe. Aux fils qui doivent, pendant le tissage, supporter sur le métier une tension forte (ceux dits de châine, comme nous le verrons), il faut donner plus de cohésion et de résistance qu'aux fils dits de trame. Alors c'est une question de dosage, pour ainsi dire, avec des points de repère établis par l'expérience, et ce travail en réalité fort complexe du moulinage qui nécessite usines, outillage et personnel, le tout spécialisé, s'exécute fort régulièrement, créant toute une gamme de fils de soie plus ou moins retords, ouvrés: organsin, trame, grenadine, poil, crêpe, marabout, cordonnet, milanaise, ondé, etc.

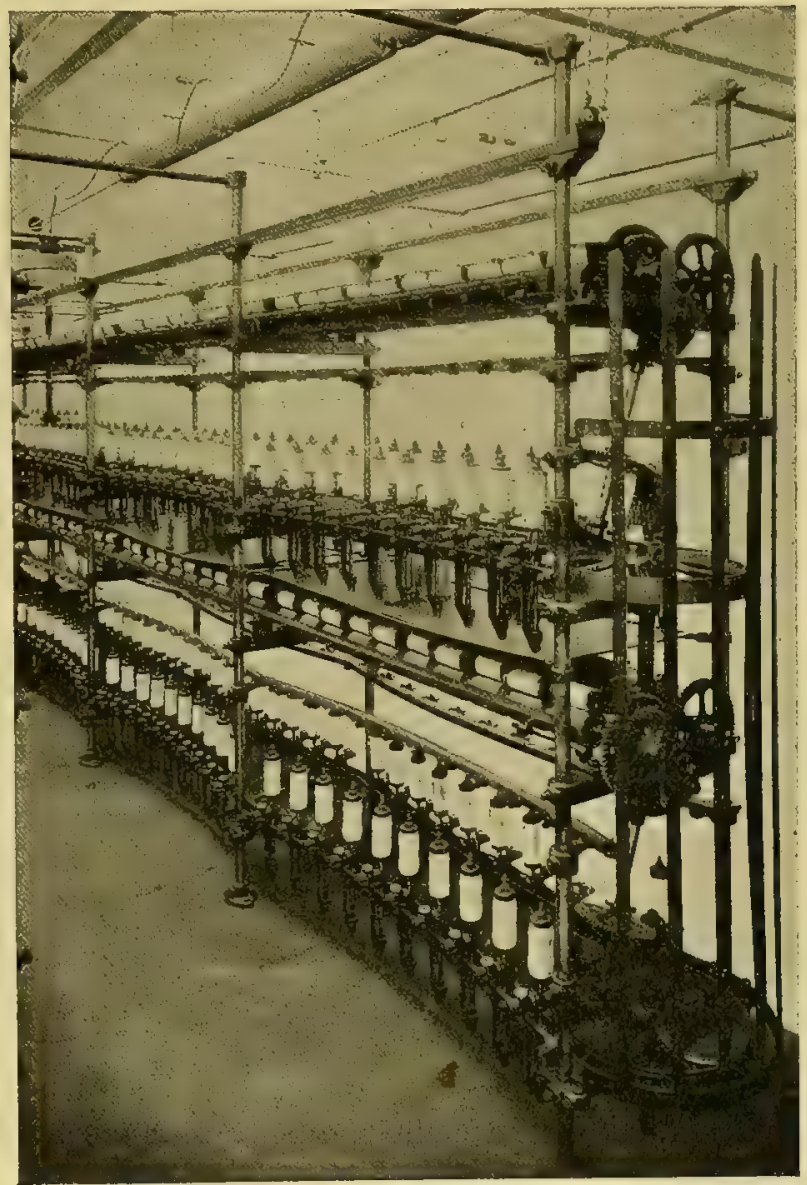

Moulinage DE LA SOIE 1

Tout cela garnit les placards du fabricant et forme des réserves très détaillées qui comprennent encore les textiles qui s'emploient assez souvent en mélange avec la soie, coton, laine, lin, schappe ou fantaisie, ces deux derniers fils tirés des déchets produits à la filature des cocons dont nous avons parlé et dont les emplois ont pris un développement très important. Les chimistes ont même voulu augmenter cet arsenal en faisant mieux encore, avec des procédés qu'il n'est pas possible de passer sous silence. Ils ont fabriqué artificiellement de la soie.

1 Communiqué par M. H. Bertrand, à Lyon. 
Partant de ce principe que la matière constituante de la soie, la fibroïne, n'est autre que de la cellulose, une cellulose, car on en distingue plusieurs en chimie, paraît-il, qui se dissout dans certains liquides, on s'est demandé si inversement, en dissolvant dans un liquide approprié de la cellulose sous forme de déchets de coton, de pâte de bois, etc., on ne pourrait ensuite extraire de la dissolution cette même cellulose sous la forme de filaments soyeux.

La question a été résolue depuis assez longtemps déjả. Il est possible d'obtenir par un travail de laboratoire des fils comparables à des fils de soie, très brillants, ayant toute la régularité et la continuité que donne la filière qui les produit, a ppareil plus rigoureux peut-être à ce point de vue que l'organe $\mathrm{du}$ ver à soie et les doigts de la fileuse. Mais ces avantages sont fortement balancés par les défauts ou les insuffisantes qualités de cette nouvelle matière, dont on aurait pu penser un instant qu'elle allait concurrencer très sérieusement la soie naturelle. Lorsqu'il fut trouvé, ce fil artificiel était très inflammable, brûlant mieux que l'amadou, presque aussi bien que le fulmicoton avec lequel ses liens de parenté étaient étroits; on remédia promptement à cet inconvénient capital, mais on ne parvint pas d'ailleurs à donner à cette soie la solidité et l'élasticité de celle produite par le Ver; lourde, et plus dense, la soie artificielle est incomparablement moins résistante à 1 humidité et à la traction. A grosseur égale et même supérieure, elle ne peut supporter tous les efforts du tissage et ne convient trop qu'à certains emplois limités. Son coût est relativement élevé du reste, car sa fabrication est chose délicate et comporte de larges installations établies sur les bases industrielles modernes, fort dispendieuses.

Cependant, la soie nouvelle a trouvé une consommation tout à fait importante pour la confection des galons, dentelles, soutaches, broderies, tous articles qui emploient un fil gros, solide, et pour lesquels son brillant spécial, très accentué, presque métallique, est une supériorité indiscutable.

C'est la cause de l'essor véritablement très considérable qu'a pris assez récemment la fabrication industrielle des soies artificielles. Il existe du reste maintenant quantité de procédés différents pour produire de la sorte des fils imitant la soie, en France et un peu partout.

On ne peut pas présager ce que sera l'avenir de cette industrie: la science sans doute réserve des surprises; il est très possible que l'on arrive à donner à cette soie artificiellement obtenue la solidité qui lui fait défaut. En attendant, la soie récoltée naturellement est d'une abondance qui rend son prix très moyen, et l'on ne voit pas qu'il y ait lieu de s'attendre à ce renchérissement qui créerait avec le coût des imitations chimiques en question, un écart tout en faveur de ces dernières.

Nous avons dit que les soieries se fabriquaient en beaucoup de cas, et presque les plus nombreux, maintenant, avec des fils de soie restés écrus, pour être teintes en pièces, celles-ci une fois fabriquées, contrairement au système séculairement suivi de tisser en teint, en employant la soie ayant reçu la couleur à la teinture en flottes.

Convenait-il donc, en se conformant à cette évolution, de parler fabrication avant d'aborder la teinture? Il nous a paru préférable cependant de rester dans l'ordre des anciens errements et ne pas repousser plus loin le chapitre de cette dernière opération très importante. 


\section{TEINTURE DE LA SOIE}

La teinture de la soie, ou en soies, n'est plus la chose relativement simple qu'elle a pu rester fort longtemps. Héritier de procédés empiriques très anciens, le teinturier d'autrefois avait aussi l'habileté du tour de main, dans une profession où des usages réglementés maintenaient les traditions. De plus, il n'avait à sa disposition qu'un nombre limité de matières colorantes, pour la plupart empruntées au règne végétal, comme l'indigo, la garance, la cochenille, le carthame, la gaude et le pastel, par exemple, dont l'emploi était encore courant il n'y a pas très longtemps.

La teinture a suivi pour sa part le progrès ou la transformation des industries textiles; ses procédés, comme ses ressources, se sont transformés de même entièrement. Pour la soie, plus ou presque plus de ces installations peu compliquées de ces petits ateliers où l'expérience et le savoir professionnel, transmis de père en fils, suppléaient à l'insuffisance des moyens matériels.

Dans les vastes usines actuelles, l'eau, la vapeur circulent en abondance, les moyens mécaniques réduisent au minimum les procédés manuels; on se sert de machines pour teindre, laver, sécher la soie; de même pour la tendre, l'étendre, l'étirer, la brillanter, l'assouplir, etc.

Quant aux substances colorantes dont on use, elles forment un lot sans cesse grandissant et infiniment varié.

La découverte de l'aniline et peu après de la fuchsine, celle-ci trouvée, en 1858 , par le chimiste Verguin, fut le point de départ d'une véritable révolution dans la chimie tinctoriale. Très vite, à ces deux produits extraits du goudron de houille, vinrent s'adjoindre quantité d'autres dérivés chimiques du même ordre, aux noms scientifiques assez barbares. Tous les jours cette série innombrable s'augmente de ceux trouvés et composés dans des laboratoires spécialement organisés pour cette recherche; l'arsenal du teinturier moderne est extrêmement vaste.

Peut-on dire que l'on teint mieux de la sorte que par le passé? Certes les nuances que l'on obtient sont chatoyantes et flatteuses. Elles ont la supériorité du nombre, la recherche des teintes, de l'imprévu même sur lancienne palette assez restreinte forcément, comme dit ci-dessus. Mais malheureusement le désavantage marqué des couleurs obtenues par les moyens nouveaux est de passer souvent avec grande rapidité en se fanant au soleil, à l'air, à la lumière du jour même, comme des fleurs fugitives. Et les vieilles soies patinées par le temps, ou demeurées parfois étonnantes de fraîcheur, attestent au contraire la solidité de leurs couleurs exigées bon teint ${ }^{\prime}$.

1 L'antiquité avait le secret des teintures extra-solides, si l'on s'en rapporte à des exemples comme celui trouvé dans Pline, livre VIII, chap. 48 , des robes prétextes dont Servius Tullius, sixième roi de Rome, fit revêtir la statue de la Fortune et qui durèrent jusqu'à la 
La chimie est encore responsable d'autres pratiques qu'on est en droit de regretter. En utilisant la faculté d'absorption très grande que possède la soie pour certaines substances, elle a trouvé la charge; ce système, qui consiste à incorporer au fil de soie, pour en augmenter le poids et aussi la grosseur, du tannin par exemple ou des oxydes métalliques, n'est évidemment pas pour améliorer la solidité, ni la résistance des tissus qu'on en fait. S'il a trouvé, peu à peu, après ses premières applications, qui remontent à une cinquantaine d'années, un notable développement, c'est qu'il a été et qu'il est le moyen le plus simple, le plus tentant de satisfaire à la recherche obstinée du bon marché pour l'étoffe de Soie. Sous l'aiguillon de la concurrence et pour offrir toujours l'occasion sensationnelle qui attire la clientèle, on est arrivé à donner à celle-ci le bas prix qu'elle réclame et en lui fournissant quelque chose dont elle a strictement pour son argent. C'est du reste une erreur considérable et une contradiction de la réalité que d'affirmer, comme nous l'avons vu imprimé quelque part, l'existence de soieries ne contenant pas plus de 5 pour roo de soie contre 95 pour Ioo de matières minérales.

Lidée de donner une augmentation de poids au fil de soie s'explique en quelque sorte par la perte de poids qu'il subit au cours de l'opération fondamentale et par laquelle, dans la majorité des cas, commencent tout d'abord les opérations de teinture. C'est le Décreusage ou Cuite qui consiste à maintenir, pendant un certain temps, les flottes écrues ou les pièces de soie tissées avec des fils écrus dans un bain de savon bouillant convenablement dosé. Cette préparation jouit de la propriété de dissoudre la partie du fil de soie écru, qui en constitue comme la gaine protectrice; c'est en réalité une sorte de vernis pigmentaire, coloré comme nous l'avons dit en parlant des cocons, et que l'on appelle aussi le Grès de la soie.

Débarrassée de cette enveloppe, la soie apparait avec tout son brillant et ses qualités spéciales, mais, forcément, à ce décreusage, elle perd une notable partie, quelquefois un quart environ, de son poids primitif. La soie se vendant au poids, on conçoit le renchérissement qui en découle sur le prix payé pour le fil grègè ou écru, ce dont il faut bien tenir compte.

A près la cuite, les flottes de soie ou les pièces sont prêtes à être plongées dans des bains de teinture convenablement composés et appliqués; elles y reçoivent les coloris que l'on a désignés d'avance au teinturier. A lui d'obtenir, par des comparaisons constamment faites avec l'échantillon qu'on lui a remis de la nuance désirée, la meilleure conformité possible. C'est souvent une tâche difficile pour les ouvriers coloristes, qui exige, avec un ceil exercé, des tâtonnements et des recommencements patients; il en faut si peu pour qu'un ton présente avec un autre une différence presque imperceptible et cependant appréciable. Les dégradations des teintes sont infinies, et les cartes de nuances, tables ou gammes de couleurs, classées et numérotées, comme il en existe par exemple à la manufacture des Gobelins, ne donnent par leur variété qu'une faible idée de celle qui existe dans la Nature.

La difficulté est souvent aussi dans la réussite de ces nuances indécises, dites rabattues ou composées, que la Mode, avide de nouveautés, a souvent

mort de Séjan, ministre et favori de Tibère, cinq cent soixante années exposées à l'air actif de cette partie de l'Italie, sans être endommagées et sans que les couleurs aient souffert! 
fait préférer aux nuances pures, franches, parfois accentuées, telles qu'elles furent longtemps en honneur. Dans ce domaine extrêmement éclectique du choix des couleurs et de leur appellation, les pourpres, les bleus azur, inde, turquin, les écarlates, les verts, les jaunes, empruntés à l'Orient, régnèrent longtemps dans l'antiquité, au Moyen Age, sous la Renaissance, et devinrent plus'tard, au XVII et au XVIII ${ }^{e}$ siècle, des gammes et des harmonies souvent fort vives; car les tons qu'on est convenu d'appeler anciens ne correspondent pas, patinés par le temps, à ce qu'ils étaient primitivement, et lorsqu'on

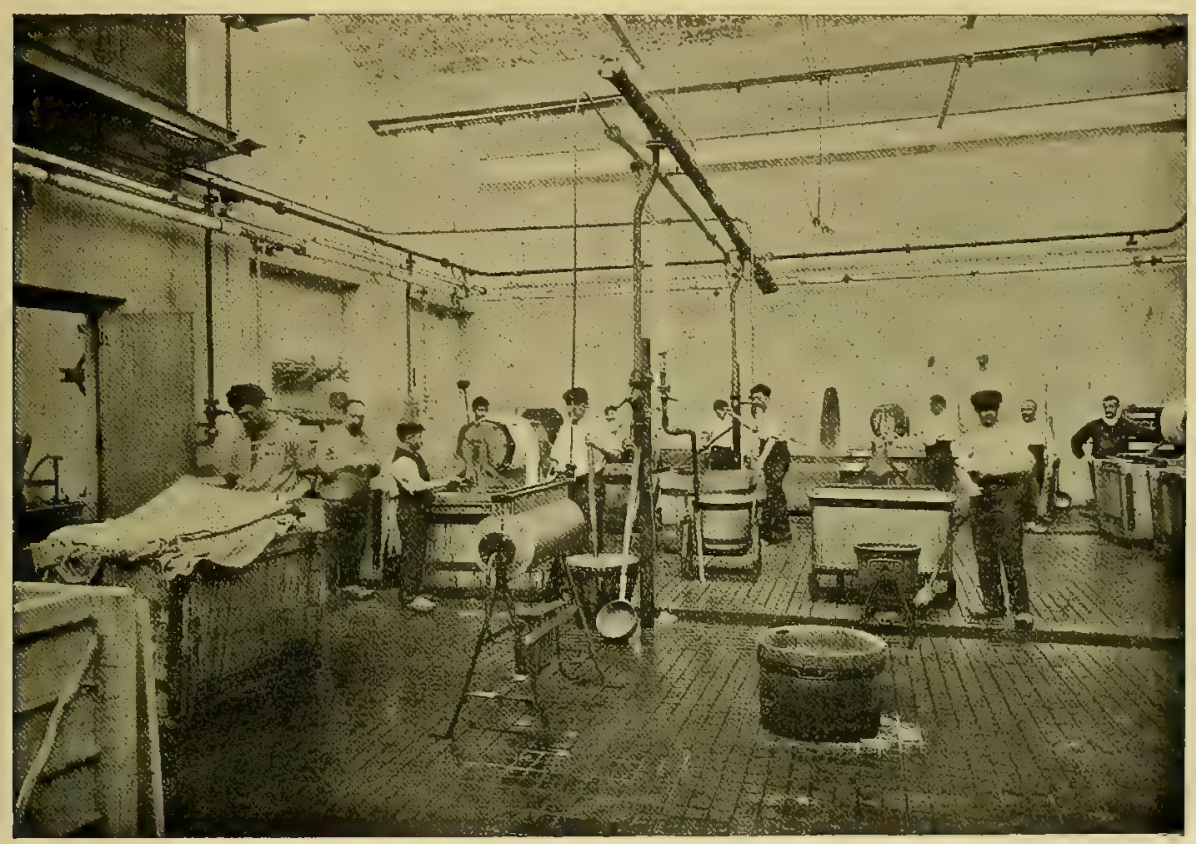

Ateler De teINTURe de soleries ${ }^{1}$ (teinture en pièces).

retrouve intacts, protégés par un heureux hasard, un velours vénitien, un lampas Louis XIV, on est frappé de la coloration très soutenue, quelquefois éclatante, qu'ils présentent. Nos pères, sous l'Empire, puis la Restauration, connurent les cramoisis, les aurores, les jonquilles, les tabacs d'Espagne et les nuances romantiques. On en est aujourd'hui à des contradictions et à de fréquents changements, surtout, bien entendu, pour les étoffes de soie destinées à faire des robes. Avec les coloris atténués, dits pastels, contrastent parfois les nuances dont le ton souvent criard et cru fait l'originalité et détermine le succès éphémère; toutes ces teintes empruntent leur appellation à un vocabulaire de saison et d'actualité qui dure ce que veut la Mode. Rapidement, tel bleu Nattier cède la place aux gris Tête de Nègre, Taupe ou Fumée, puis à un Kaki, un Prunelle, etc., etc., et à la teinte qui fera fureur demain et qu'il faut renoncer à prévoir!

1 M. Henry Bertrand, Lyon. 
Croirait-on que le blanc et aussi le noir, nuances fondamentales, sont pour la soie celles qui nécessitent les soins les plus minutieux et les pratiques les plus exercées, sans qu'on puisse arriver toujours à réaliser scrupuleusement la conformité d'une teinture à une autre. Malgré tous les blanchiments, la composition intime du fil de soie et l'imprévu jouent leur rôle et créent les différences que présentent ces blancs entre eux, surtout perceptibles à ceux dont c'est le métier, et qui ont leur cause dans le jeu inégal de la lumière différemment réfléchie ou absorbée.

Les noirs, en effet, détiennent peut-être le record de la variété. Rien ne ressemble moins à un noir qu'un autre noir; la soie ne fait pas exception à ce fait facile à vérifier, et la difficulté du réassortiment des étoffes noires est bien connue des couturières. Alors on réclame, on recommence et on procède à des refaisages, et bien souvent on ne fait pas mieux: plat réchauffé ne valut jamais rien!

Voici cependant, sorties des usines de la teinture, les flottes de soies diversement colorées, selon ce que le fabricant a demandé, ayant subi en plus les traitements appropriés aux emplois en vue, car le tissage de divers genres d'étoffes exige de la soie plus ou moins cuite, plus ou moins assouplie, plus ou moins avivéc, c'est-à-dire rendue craquante, ou préparée pour des manipulations ultérieures, telles que l'Impression, la Moire, etc., etc.

Ou bien, au lieu de flottes de soie, ce sont, nous le rappelons, les pièces mêmes d'étoffes qui sont rendues toutes prètes et teintes, pièces que l'on a tissées avec des soies écrues et dont la manipulation est devenue dans la branche teinture une spécialité d'importance croissante, quoique reposant évidemment sur des procédés analogues à ceux du traitement de la soie en flottes '. A ce dernier point de vue, il y aurait lieu d'en venir dès maintenant à l'opération importante qui suit nécessairement la teinture de ces pièces de Soie, à l'apprêt qui les finit et les fait telles qu'on les offre à la clientèle, mais il nous semble néanmoins normal de suivre auparavant, dans cet exposé, l'ancien ordre de choses et d'aborder tout de suite la question du tissage proprement dit, si bien quion puisse avoir une idée de la façon de fabriquer des pièces sans distinction de catégories, avant de voir comment on les apprête, quand il convient quelles soient ainsi traitées.

1 Il faut noter encore les manipulations toutes spéciales qui conviennent pour la tcinture des soies pour Moire, des soies dites «solides à la cuite » dichroïques ou à reflets, et des soieries mélangées, telles que soie et laine, soie et coton; dans ce dernier cas, par l'emploi de colorants distincts et appropriés, on teint fort bien le coton, matière végétale, d'une nuance, et la soie, matière animale, d'une autre différente. Mieux encore: un procédé récent donne le moyen, en préparant de la soie à l'avance, et en employant dans le tissu soie non préparée et soie préparée, de donner à chacune d'elles une couleur différente : ce système de la double teinte, par teint en pièces, donne des résultats surprenants et fort commodes en beaucoup de circonstances. 


\section{FABRICATION}

\section{DÉVIDAGE - OURDISSAGE}

Élémentairement, le problème du tissage consiste, on le sait, à entremêler, à entrecroiser des fils, de telle sorte que résulte de cette agrégation une étoffe, un tissu. Ce travail s'exécute au moyen du métier, comme chacun le sait; et la notion fondamentale qui s'impose à l'examen tout de suite, quand on parle métier à tisser, est celle de la distinction des fils employés sur ce métier, suivant leur rôle et leur ordre, au tissage en fils de chaine et fils de trame; les premiers sont ceux disposés, tendus en long, les uns à côté des autres, en nappe sur le métier, un peu à la façon, si l'on veut, du canevas tendu sur le métier de broderie, tandis que les seconds, au contraire, sont passés successivement $u n$ à un par l'ouvrier au travers des autres, de telle sorte que la trame se trouve ainsi emmêlée,

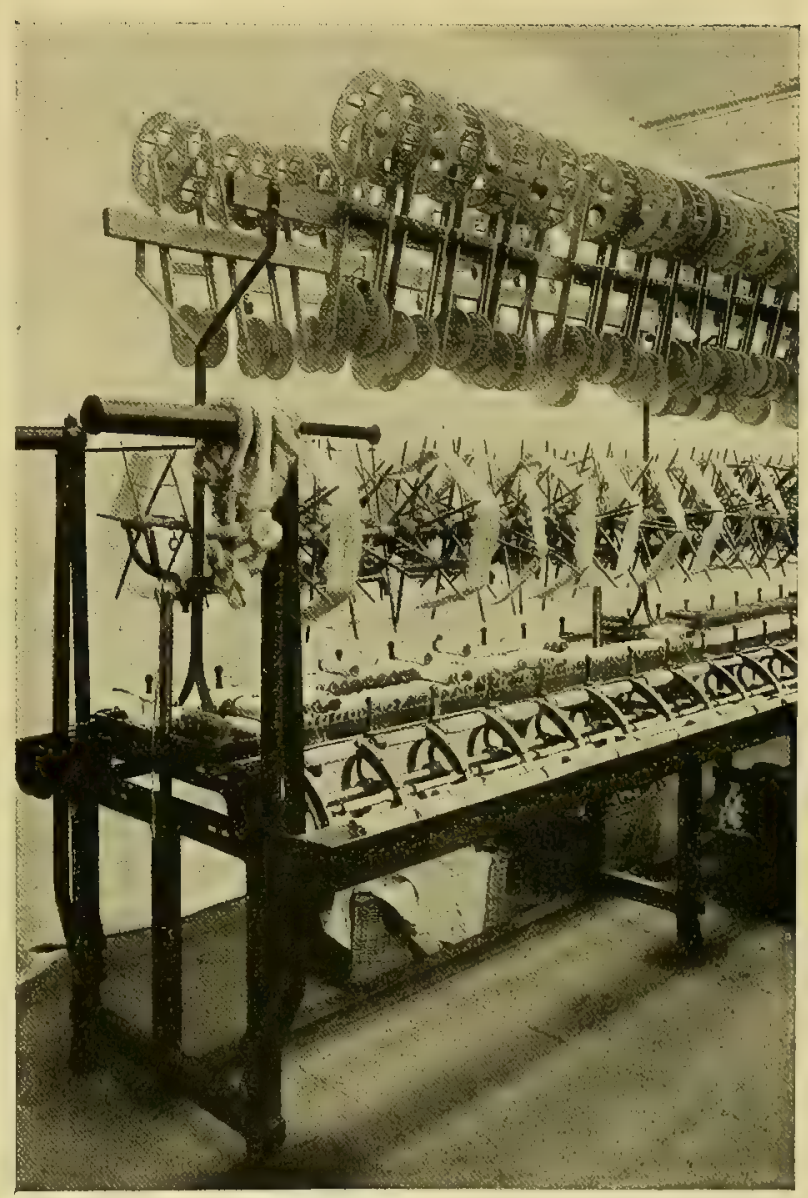

DÉvidage MÉCANIQUe de La SOIE ${ }^{1}$. coup par coup, à la chaîne, dans une direction perpendiculaire à celle de cette chaîne.

Les fils de soie teints ou écrus, jusque-là en flottes, doivent bien entendu, à cette fin de tissage, être enroulés sur des bobines, grosses ou petites, en bois ou en métal, par un dévidage qui s'opère aujourd'hui en grand et ne

1 M. Henry Bertrand, Lyon. 


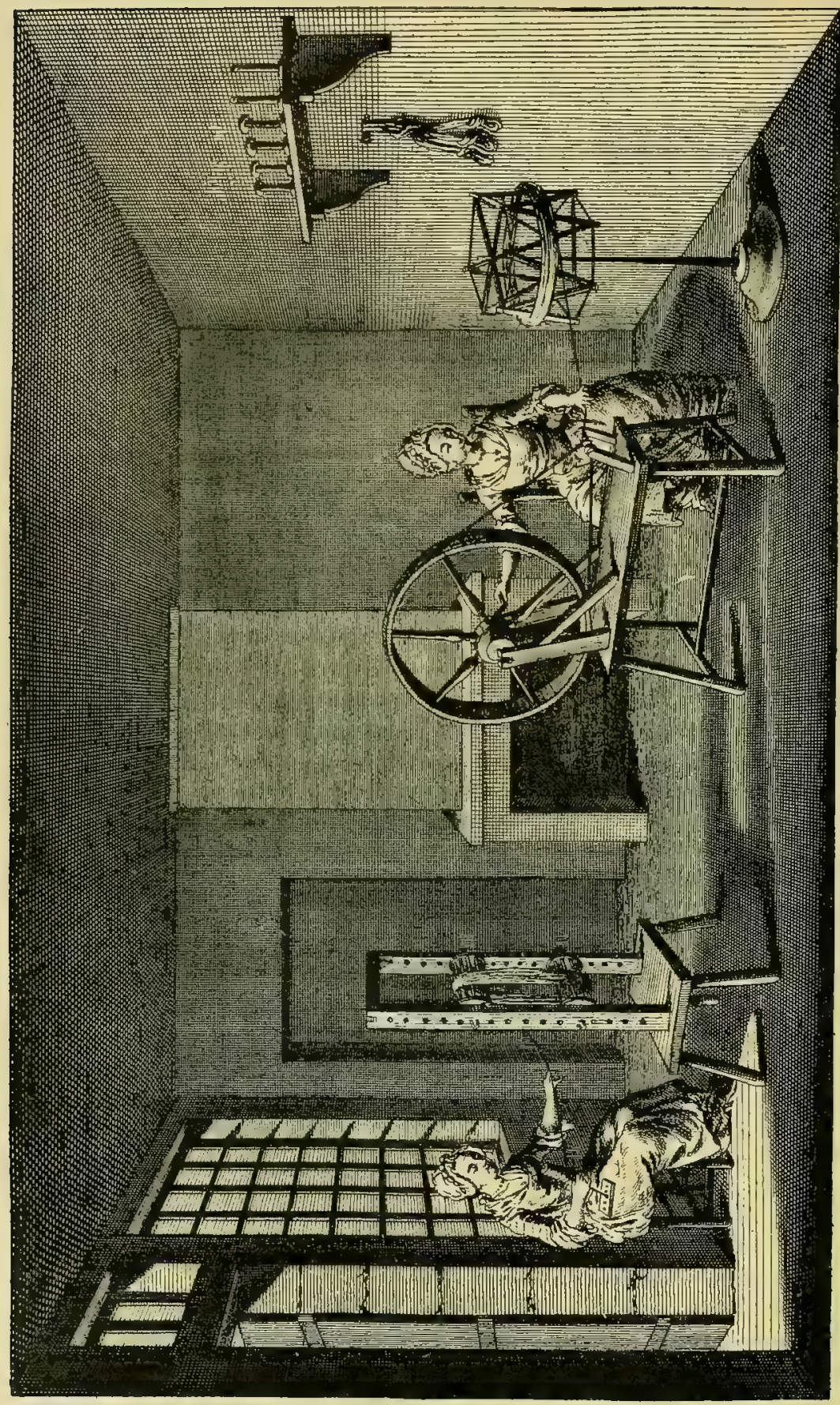

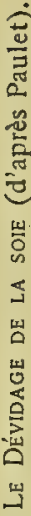


rappelle que de loin la manière toute primitive dont lienfant aide, sur ses mains tendues, au dévidage de l'écheveau de sa grand'mère.

Bobines de trames ou de fils de chaîne sont étiquetées, pesées, comptées, répertoriées soigneusement.

On se servira des premières pour garnir, le moment venu, de plus petites bobines, cannettes ou tuyaux, assez étroites pour être placées à l'intérieur de la navette, instrument mobile qui portera la trame au travers de la chaîne.

Quant à celle-ci, il faut encore l'ourdir. Ourdir est un mot qu'on rencontre à chaque instant dans le langage littéraire employé, d'une manière dont lusage a consacré l'inexactitude.

Quand Figaro s'écrie « Ah! ma trame est bien ourdie! », il commet, en vérité, un contresens, puisque, de fait, une trame n'est jamais ourdie; ce n'est que dans les romans à sensation ec péripéties que l'on ourdit des trames odieuses, tout comme de noirs complots. C'est un exemple de curieuse déviation de sens que donne parfois le langage à des abstractions ou similitudes empruntées à des réalités objectives.

Au contraire, tramer, dans le sens de machiner, est resté une image exacte et parlante, tout comme : avoir une teinture de telle ou telle connaissance.

C'est, en réalité, la chaîne seule que l'on ourdit, car ourdir c'est justement composer l'ensemble des fils de chaîne en nombre voulu comme de longueur pareille, avec une tension égale et régulière. La longueur est celle choisie pour la pièce ou les pièces successives à tisser, en tenant compte de la perte ou déchet inévitable au commencement et à la fin du travail, et encore du retrait, ou, comme l'on dit, de l'embuvage que subissent ces fils de chaîne au tissage.

On comprend mieux ce dernier point par un exemple. Une ficelle, type du fil de chaîne, passant dessus et dessous les doigts de la main figurant les fils de la trame, représente, très grossi, le chemin sinueux des fils de chaîne dans le tissu même; par suite on imagine le raccourcissement de ces fils, qui, en fait, et selon le mode de croisement adopté, n'est jamais négligeable et atteint, pour certaines étoffes, une valeur très considérable.

On n'ourdit plus guère à la main, mais si l'accessoire séculaire et obligé du tissage est devenu un organe mécanique, partant plus précis peut-être, surtout plus expéditif, le principe est resté le même : il consiste toujours à prendre un certain nombre de bobines chargées de la soie convenable, le plus grand nombre possible évidemment, à les disposer sur des sortes d'étagères ad hoc ou cantres, qui les supportent en permettant le déroulement des fils; puis, à réunir toutes les extrémités de ces fils à un grand tambour de circonférence connue, à le faire tourner jusqu'à ce que l'on ait 100, 200, 300 mètres ou plus de fils de soie enroulés; enfin, à recommencer l'opération en enroulant, de même et à côté de la première longueur mise sur le tambour, une nouvelle longueur identique du même nombre de fils sur lequel on opère; ceci autant de fois que nécessaire pour obtenir au total le nombre de fils de chaîne de la pièce et que l'on a calculé à l'avance. C'est de l'arithmétique et du soin. Un Taffetas, par exemple, dit fond de jupe, bonne qualité, en 52 centimètres, comporte, sans les lisières, environ 2.000 à 3.000 fils de chaîne, tandis qu'un beau satin Météor, pour la largeur de i Io centimètres, en contient de 9.000 à 12.000 ! 


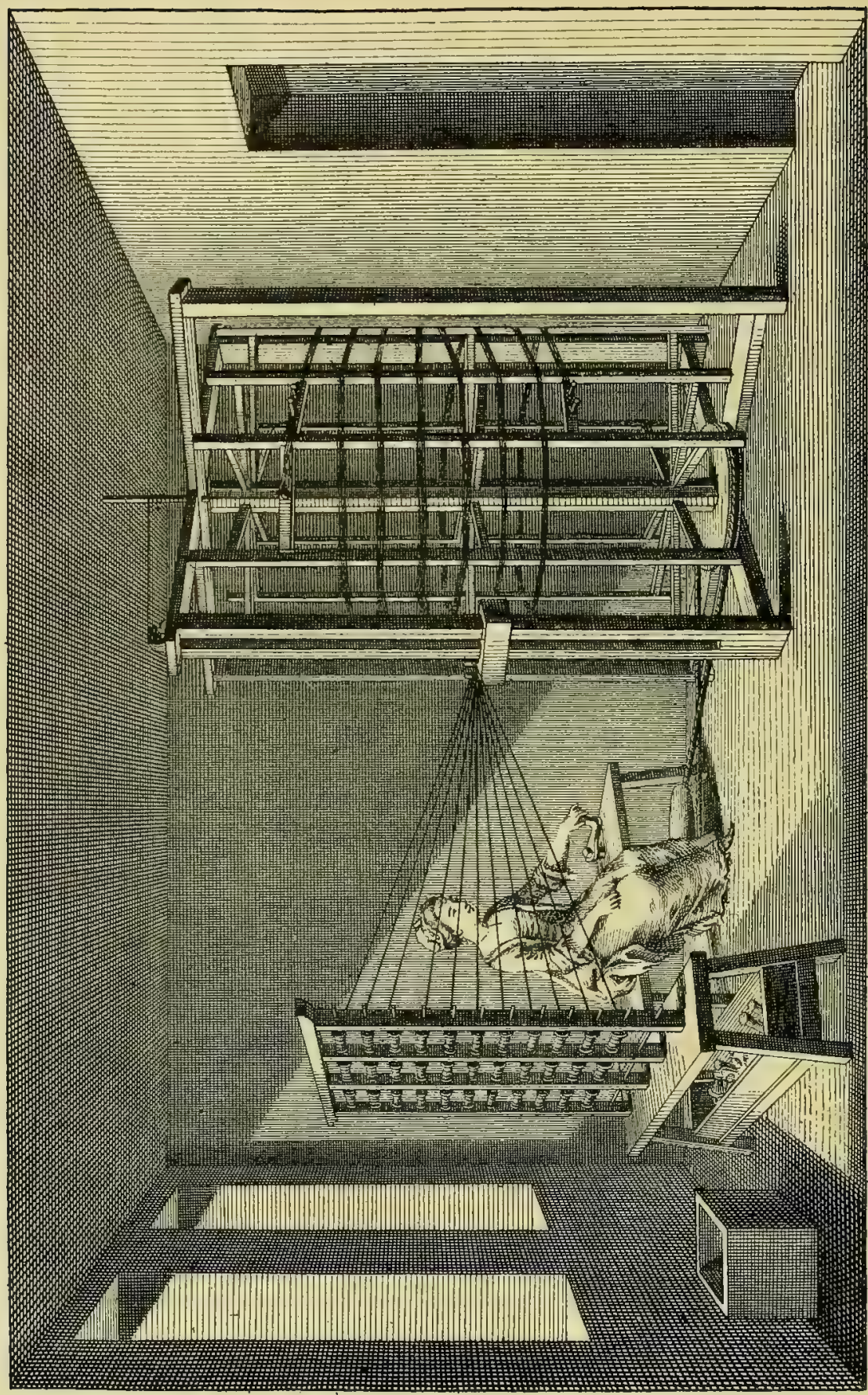


Mais l'ourdissage est encore une chose plus délicate et compliquée, quand il faut le faire, par exemple, à fils doubles, triples, etc., c'est-à-dire procéder comme si ces fils, provenant de deux, trois bobines ou plus, disposés par groupes, n'en faisaient qu'un, deux fois, trois fois.plus fort, ceci pour les besoins de l'étoffe que l'on veut tisser; ou bien encore quand il s'agit d'ourdir des chaînes pour des soieries à bandes de couleurs ou de contextures différentes, ou pour celles comprenant des dégradations de nuances, des ombrés, des mélanges de matières, etc.

Passons sur ces minuties et mentionnons que, la chaîne une fois ourdie, une opération intervient encore, celle du pliage, qui, professionnellement exécutée, dispose enfin cette chaîne bien enroulée sur le rouleau du métier destiné à la recevoir, telle qu'elle doit èłre pour la bonne exécution du tissage ultérieur. 


\section{LE MÉTIER}

Si l'on se borne à l'envisager de façon élémentaire, le métier à tisser est un instrument de travail dont l'antique simplicité va de pair avec l'invention qui remonte aux temps les plus reculés. En principe, ses organes essentiels se réduisent à quelques-uns, quand on les débarrasse des mille et un détails dont l'ingéniosité humaine les a successivement perfectionnés et qu'on cherche à en établir la description schématique.

C'est tout d'abord un bâti rectangulaire ${ }^{1}$, formé de pièces de bois verticalement et horizontalement assemblées pour rendre le tout suffisamment rigide. Deux cylindres ou gros rouleaux de bois y sont placés et disposés symétriquement, en face l'un de l'autre et à peu près à la même hauteur au-dessus du sol, soit à peu près celle d'appui de la main; ils reçoivent les fils de la chaîne qui, enroulés d’abord complètement sur l'un des deux rouleaux, passent à l'autre en formant une nappe horizontale qu'on a le soin de maintenir convenablement tendue, tirante, comme on dit en langage de canut, par des dispositifs appropriés.

Toute la question est maintenant de faire mouvoir ces fils, de les écarter les uns des autres de façon à laisser entre eux, à un point donné de leur longueur, un espace qui permette d'y passer le fil de trame. Celui-ci, lorsque les fils de chaîne auront repris leur place primitive, se trouvera ainsi inséré dans cette chaîne, lié par elle, et formera le tissu.

Le croisement doit évidemment avoir lieu dans un certain ordre et dans une certaine proportion. Prenons le cas le plus simple, celui du Taffetas. Dans le Taffetas, les fils se croisent, comme dans la toile ou le calicot le plus quelconque, par moitié, c'est-à-dire fil à fil et inversement; en précisant, pour un premier fil ou coup de trame à passer, les fils impairs, numérotés I, $3,5,7$, etc., de la chaine lèvent, tandis que les fils pairs $2,4,6$, 8, etc. restent au-dessous ou en fond. Au contraire, pour passer un deuxième coup de trame consécutif et lier le premier, ce sont ces fils pairs qui lèvent et les fils impairs qui restent en fond.

De ce Taffetas, ou mode de croisement élémentaire et universellement connu, dérivent à peu près tous les autres, ceux qui donnent les étoffes unies sans dessins et que l'on désigne sous le nom gẻnéral d'Armures, terme assez comparable à celui de points, usité pour la broderie et la dentelle.

Or, pour obtenir commodément la manœuvre nécessaire au travail de la levée des fils en croisement donné, on se sert d'organes du métier que l'on nomme lisses dans la région lyonnaise.

Qu'on imagine des lamettes de bois dont, pour l'exemple du Taffetas, deux peuvent suffire à la rigueur; disposées horizontalement au-dessus et audessous de la nappe des fils de chaîne et à une distance chacune d'environ

1 Voir la figure, page 8. 
30 centimètres de celle-ci, elles sont reliées deux à deux par une série de fils verticaux, en coton le plus généralement, qui forment des mailles ou boucles au milieu environ de la distance qui sépare les deux lamettes de bois. Si l'on s'arrange pour faire passer successivement à l'avance tous les fils impairs de la chaîne de notre Taffetas dans les boucles de l'une des deux lisses et, au contraire, tous les fils pairs dans les boucles de la seconde, on a le moyen, en élevant l'une puis l'autre par des cordelettes et le dispositif ad hoc qui les tient suspendues à leur place sur le métier, tout en permettant ce déplacement en hauteur, d'obtenir le croisement cherché.

Ces mouvements sont commandés à l'aide de poulies de renvoi, de leviers ou marches mus au pied dans le simple métier à bras, ou enfin de systèmes plus compliqués, dits mécaniques d'armures, dont 1e fonctionnement est provoqué et réglé comme il convient par le mouvement même d'impulsion donné au métier.

Deux lisses suffisent pour le Taffetas, mais c'est le cas ultra-simple; à cause du nombre de fils de chaîne en jeu, il en faut au moins quatre et, d'après la nature et la complication des autres armures et les qua-

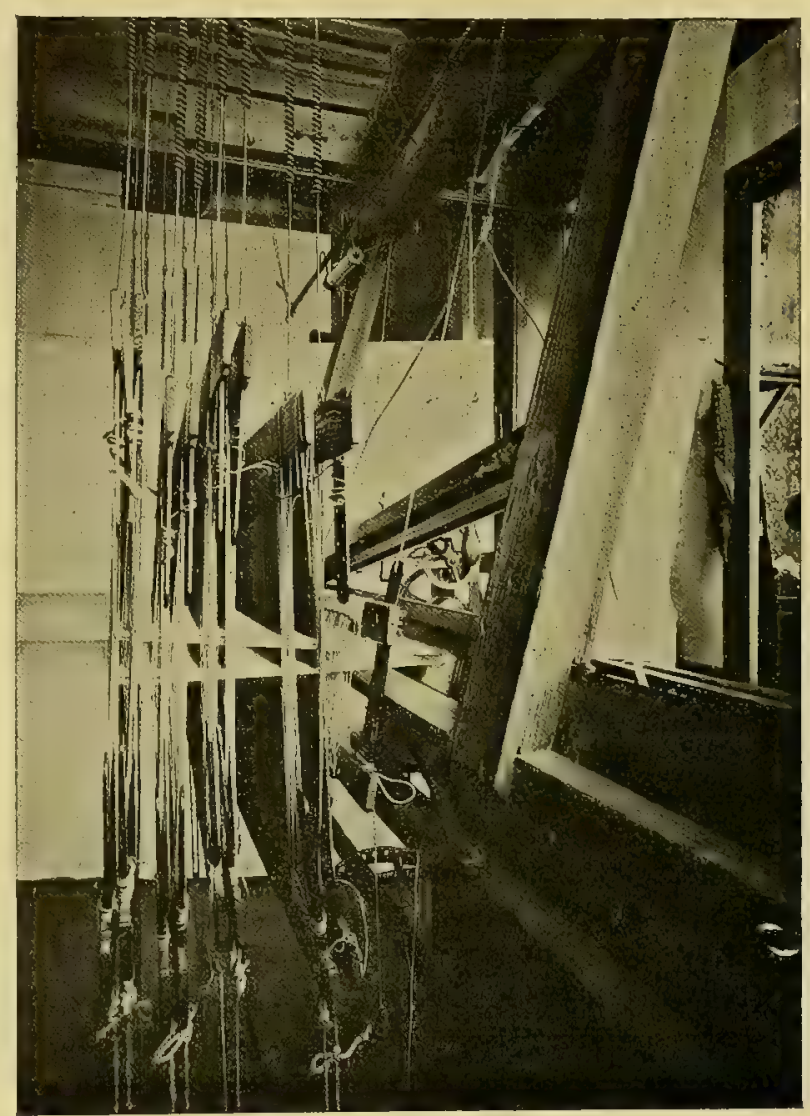

DÉTAILS DU MÉTIER : BATTANT, PEIGNE, LISSES. lités d'étoffes que l'on veut obtenir, des nombres tels que 6,8 , Io, I 2, I 6 sont nécessaires, jusqu'à même 24 et 32 en certains cas. Leur ensemble se nomme remisse, et l'opération qu'on comprend facilement ne pouvoir être confiée qu'à des spécialistes, pour ajuster les fils de la chaîne dans ces lisses, se dénomme le remettage.

En possession du moyen de préparer et de ménager à travers les fils de chaîne le passage de la trame, reste à voir comment s'opère celui-ci. Ici intervient l'instrument peut-être le plus connu entre tous ceux du tisserand et comme son emblème, la navette, morceau de bois allongé terminé en pointe à ses deux extrémités et portant en son centre un évidement où vient se loger la cannette, sorte de bobine spéciale chargée du fil de trame qui se déroule ou se défile ainsi au fur et à mesure. Pour donner à la navette 
l'impulsion nécessaire à son lancement et à ses multiples voyages, aller et retour, au cours desquels la trame s'incorpore à la chaîne, un autre organe du métier est encore indispensable et même avec une double utilité.

C'est le battant, sorte de cadre de bois assez lourd, suspendu par le haut à la partie supérieure du métier, entre les lisses et le rouleau dit de devant, en face duquel se tient l'ouvrier, de telle sorte qu'il puisse osciller sous la pression de la main gauche du tisseur, en s'éloignant ou se rapprochant dudit rouleau, selon les besoins du travail. A droite et à gauche, dans ce battant, dont la largeur dépasse forcément pas mal celle de l'étoffe à produire, des logements sont ménagés pour recevoir la navette, qui est alternativement lancée de l'un à l'autre et assez brusquement même comme il convient, par le moyen de sortes de taquets à coulisse ou rats mus par des cordelettes que des poulies de renvoi ramènent à une seule placée devant l'ouvrier, à hauteur de sa main droite, et qu'il tire au moment convenable.

Ainsi, les lisses ayant été levées dans 1'ordre nécessaire, pour passer un coup de trame, louvrier écarte le battant en le repoussant, afin que la navette, portée par ce battant, se trouve à droite ou à gauche à l'entrée du passage ménagé entre les fils de la chaîne. La navette, sollicitée par le taquet, passe en roulant ou en glissant sur les fils restés en dessous ou en fond, en s'appuyant sur une petite lame de bois feutrée portée horizontalement par le battant en dessous de ces fils, et le fil de trame s'étend à sa place.

Mais alors intervient l'ustensile auquel le battant sert également de support et dont le rôle est des plus importants.

Nous voulons parler du peigne, qui tire son nom de sa ressemblance de plus en plus éloignée avec le peigne à coiffer; on le fit, aux époques primitives du tissage, de bois, d'os, de corne, etc.; il se présente maintenant sous la forme d'une sorte de lame métallique rectangulaire de longueur variable et proportionnée à celle du métier, de l'étoffe, de largeur à peu près constante et composée de dents d'acier parfaitement polies, très serrées les unes contre les autres, avec une exacte régularité mathématique, entre deux règles de métal auxquelles leurs deux extrémités sont soudées. Le nombre de ces dents, compté dans I centimètre ou dans I pouce, vieille mesure encore très usitée, peut varier, selon les besoins de la cause, depuis 3 ou 4 jusqu'à 75 ou 80 dans I centimètre, ce qui donne une idée de la précision avec laquelle sont établis ces instruments: précision qui est indispensable, car, placé dans le battant, juste un peu en arrière, par rapport à l'ouvrier, du trajet que doit suivre la navette dans sa course, le peigne reçoit les fils de la chaine qui passent dans ses dents un à un ou presque constamment par un nombre plus élevé - 2, 3, 4, 5, 6, 8, I0, I 2, etc. - de fils dans chaque dent. Cette répartition est réglée, calculée en raison de la qualité, de l'entente et du genre du tissu cherché.

Mais, dira-t-on, pourquoi cette complication, qui paraît bien grande, de passer tous les fils de la chaîne en nombre déterminé, rigoureux, dans toutes les dents du peigne? Certes, ce piquage en peigne n'est pas une besogne aisée pour le premier venu, non plus qu'un exercice à recommander aux impatients et aux distraits. Mais les avantages de cette pratique sont que, de la sorte, les fils se trouvent régulièrement distribués sur la largeur de l'étoffe, largeur 
donnée et maintenue, autant que faire se peut, justement par le péigne. Ensuite, ce peigne mobile avec le battant, placé perpendiculairement à la direction de la chaîne qui le traverse, va, en se rabattant vers l'ouvrier lorsque celui-ci ramène à lui le battant avec une force calculée, faire se joindre contre les précédents coups de trame déjà passés et formant l'étoffe, le dernier fil que la navette a laissé derrière elle à son passage. La régularité du tissu se trouve donc assurée, puisqu'elle dépend, en première ligne, de la juxtaposition correcte des coups de trame les uns à côté des autres.

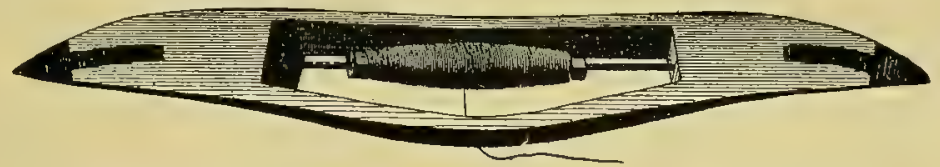

Navette ancienne lancée a la main.

C'est ce que l'on nomme la réduction du tissu; elle est prévue d'avance suivant la grosseur de la trame et le genre que l'on veut fabriquer.

Lorsque l'ouvrier commence à tisser la pièce, il fait d'abord une petite partie d'essai de quelques centimètres : c'est la tirelle qui permet de voir si tout va bien et se trouve bien réglé dans le métier, ainsi que de juger des corrections nécessaires. La mise en route une fois faite, le tissu s'enroule au fur et à mesure de sa fabrication sur le rouleau placé devant l'ouvrier; nous verrons à nouveau, à propos des étoffes à dessins, qu'un des organes les plus nécessaires du métier est celui qui régit cet enroulement continu, de telle sorte que les fils ou coups de la trame se placent toujours bien régulièrement les uns à la suite des autres, sans variations trop sensibles, qui apparaîtraient forcément sur l'étoffe plus ou moins, selon sa contexture, mais en la dépréciant toujours.

Il faut, comme l'on dit, que l'étoffe soit battue régulièrement ou que le

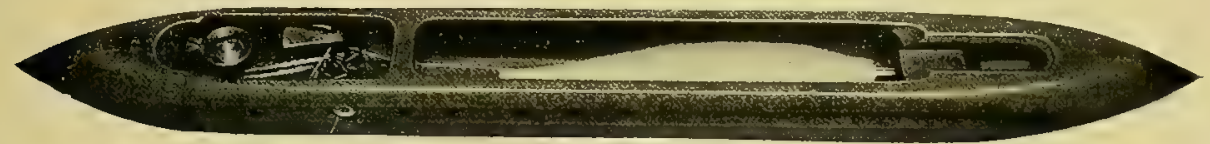

NAVETTE MODERNE EMPLOYÉE POUR LE MÉTIER MÉCANIQUE.

battage soit bon. Inversement, le tissu est varié, entrebattu, mêlé de claires ou serrées, expressions qui parlent d'elles-mêmes.

Une quantité d'autres défauts, du reste, peuvent se présenter sur le tissu, que l'ouvrier doit prévoir et éviter de son mieux. Un ou plusieurs des fils si légers, somme toute, de la chaîne, peuvent casser, et, si on ne les renoue ou rhabille pas aussitôt, on voit sur l'étoffe la trace de ces fils manquants, laquelle devient un crapaud, ou une grille, si plusieurs fils ont cassé à la même place et se sont embrouillés. D’autres fils peuvent être entraînés, levés intempestivement, occasionner des tenues, des groupures, des piqûres. De la trame mal surveillée ou irrégulière viennent les bouchons, costes, arbalètes, trames doublées, trames tirantes, etc., etc. 
Enfin, du travail même de l'ouvrier qui peut avoir de l'inexpérience, des inattentions, et par accident, résultent encore d'autres défauts, tels que, par exemple, un pas failli, ou erreur dans l'armure, venant d'un coup de trame oublié ou qui n'a pas été passé à son rang, et bien d'autres encore.

On voit qu'il faut, pour le travail délicat du tissage de la soie, beaucoup d'attention, de surveillance, en même temps qu'un entraînement très habile, d'autant que, la plupart du temps, ces soieries, qui comportent un endroit et un envers, se font, pour des raisons d'ordre pratique, endroit dessous, et l'ouvrier voit se former devant lui l'envers du tissu. D'où nécessité, parfois, les défauts n'apparaissant pas, dans certains genres, de ce côté de l'étoffe, de suspendre le travail, de dérouler ce qu'il y a de tissé, en faisant lâche, expression typique, qui signifie que l'on a fait cesser momentanément la tension de la chaîne, de façon à pouvoir procéder à l'examen en question.

Et, en cas de défauts trop marquants, il faut, quand c'est possible, défaire ou détisser la partie du tissu défectueuse et la recommencer, ce qui n'est pas toujours très facile et commode et implique toute la dextérité que donne une patiente pratique.

Autre question importante dans le tissage : le soin à donner aux lisières ou cordons; les fils qui les composent sont presque toujours plus fournis, plus serrés que dans le fond même du tissu, et souvent se trouvent encore mis de couleurs tranchant sur ce fond, de façon à en être comme l'encadrement. On attachait autrefois beaucoup d'importance à la parfaite exécution de ces lisières, ce qui dénotait, en général, le mème soin pour l'étoffe elle-même. Les usages corporatifs réglementaient scrupuleusement ce détail, aussi bien que la largeur des différentes soieries, qui n'était pas chose facultative, mais bien soumise à la surveillance des Maîtres Gardes veillant à l'observation stricte des règlements, prescrivant les Io/ $12^{\text {es }}$ ou les I I $/ 24^{\text {cs }}$ d'aune, suivant le cas.

Aujourd'hui, la largeur varie avec les besoins et les emplois. Telle forme de costume déterminera, par exemple, la prédominance de la petite ou de la grande ou double largeur avec leurs variantes : 50, 52, 54, 60 centimètres et 80 , I 00 , i 10 et I 20 centimètres sont des mesures fréquentes. On conçoit que l'ameublement, le parapluie, la doublure, plus encore le ruban en réclament de particulières ou même très spéciales. Quant à la longueur des pièces, c'est également une convention dictée par l'usage qui la fixe actuellement.

Les pièces une fois terminées, tombées du métier, reste à les soumettre à une visite ou examen minutieux sur toute leur longueur, à un pincettage ou enlèvement de défauts, aspérités, bouchons, à l'aide de petites pinces ou pincettes et des ciseaux de forme spéciale, appelés forces, dont on a coutume de se servir pour le tissage.

Avant de mettre les pièces de soie à la vente, pliées, enveloppées, encartonnées, roulées, etc., ainsi que les usages le réclament, il faut encore leur faire subir des opérations appropriées à chaque genre d'étoffes et dites de finissage; le but en est de donner au tissu son aspect, son toucher et sa présentation définitive, tout cela d'après les enseignements de l'expérience et réalisé par de multiples moyens.

Aujourd'hui ces pratiques ont même atteint un développement si considérable que ce n'est pas trop d'en parler dans un chapitre à part.

Au cours des lignes ci-dessus, il a été nécessaire d'insister sur des détails 
assez techniques, qu'il était impossible de laisser de côté, néanmoins, pour l'intelligence du sujet, et pour donner une idée d'ensemble et générale du tissage en lui-même.

Nous avons cependant négligé, à dessein, quantité d'autres points plus précis; et si nous nous décidons à passer encore sur la description, qui devrait prendre place ici, des nombreuses sortes de métiers existant maintenant pour tisser la soie, résultats de perfectionnements venant s'ajouter les uns aux autres à travers les siècles, c'est que cette question nous entraînerait forcément en dehors du cadre que nous nous sommes proposé. Tout au plus sera-t-il possible de donner çà et là quelques renseignements de cet ordre à propos des différents genres de soieries et des moyens usités pour leur fabrication. 


\section{LES INDUSTRIES DE FINISSAGE}

Ce n'est pas d'hier que l'on a reconnu l'utilité et même la nécessité de finir, en quelque sorte, l'étoffe, une fois fabriquée ou tombée du métier, selon une locution très employée; ceci pour effacer les plis formés accidentellement, donner une tension régulière à cette étofte et aussi un toucher meilleur. Chacun sait le rôle très important de l'apprêt, terme générique dont le sens est bien connu, en particulier pour l'achèvement des tissus de laine et de ceux de coton.

Les soieries, pour la plupart, demandent aussi à être achevées, finies, et il existe à cet effet une notable variété de manipulations adaptées à leurs différents genres.

Certaines étoffes de soie, comme celles à grains, à côtes, prođuits par la trame, se trouvent parfaitement d'abord de l'opération du polissage, que l'on a pratiquée longtemps au cours de la fabrication et sur le métier même avec le polissoir; c'est un instrument formé d'une lame semi-elliptique de corne ou d'acier, fixée à une poignée de bois que l'ouvrier tient à la main. En frottant en long et en large la partie de l'étoffe que l'on vient de tisser, laissée assez lâche, avec le bord de cette lame maniée adroitement et soigneusement, on fait se disposer plus également les fils entre eux et le tissu acquiert plus de régularité, plus de couverture, avec une souplesse plus grande.

De même fut remarqué, il y a longtemps, le résultat très favorable obtenu en faisant passer les mêmes étoffes très tendues sur des cylindres ou rouleaux métalliques chauffés exerçant, par leur frottement, une action comparable à celle d'un fer à repasser. C'est ce que l'on nomme le cylindrage qui donne du brillant, du lustre à ces étoffes, et en améliore le toucher souvent assez $\mathrm{sec}$, cassant, avec trop de carte, lorsqu'elles viennent du métier.

Il fut un temps où les taffetas étaient lustrés par un procédé imaginé par un Lyonnais notoire du XviI siècle, Octavio Mey. Le hasard, paraît-il, lui en avait donné l'idée. On raconte que ruiné, accablé de déceptions et de soucis, il se promenait un jour en mâchonnant machinalement quelques brins de soie, qu'il avait portés distraitement à sa bouche. Il lui arriva de porter ses yeux sur ces fils et remarqua le brillant et l'aspect particulier qu'ils avaient pris sous l'action combinée de l'humidité et de la chaleur de la salive, en même temps que de sa composition et de la pression exercée par les dents. Ces observations l'amenèrent à tenter des essais pour réaliser des opérations analogues et appropriées sur des pièces entières de Taffetas, ce qui réussit au delà de ses prévisions. Octavio Mey trouva dans l'exploitation de ce lustrage les éléments d'une nouvelle fortune; ce procédé, du reste, a été tout à fait abandonné depuis lors.

Certaines soieries, sans doute, celles surtout tissées en teint en fils et de belles qualités, peuvent rester, à peu de chose près, telles que le métier les a produites; des moyens très simples, comme un enroulage soigneux, 
suffisent à les tendre convenablement. Mais, pour la généralité des autres, au contraire, il faut faire intervenir l'apprêt. Les soieries teintes en pièces, dont la production grandit de jour en jour, le réclament nécessairement. C'est l'apprêt qui donne au tissu l'aspect, le toucher, l'épaisseur recherchés, et qui le transforme mème, dans certains cas, du tout au tout, et de sortes de chiffons légers, sans consistance, inutilisables et imprésentables, fait les Mousselines, les Gazes, les Crêpes, les Crêpons ou les Tulles, tels qu'on a coutume de les voir.

Il y a des soieries à dessins, comme certains Damas, qui s'accom-

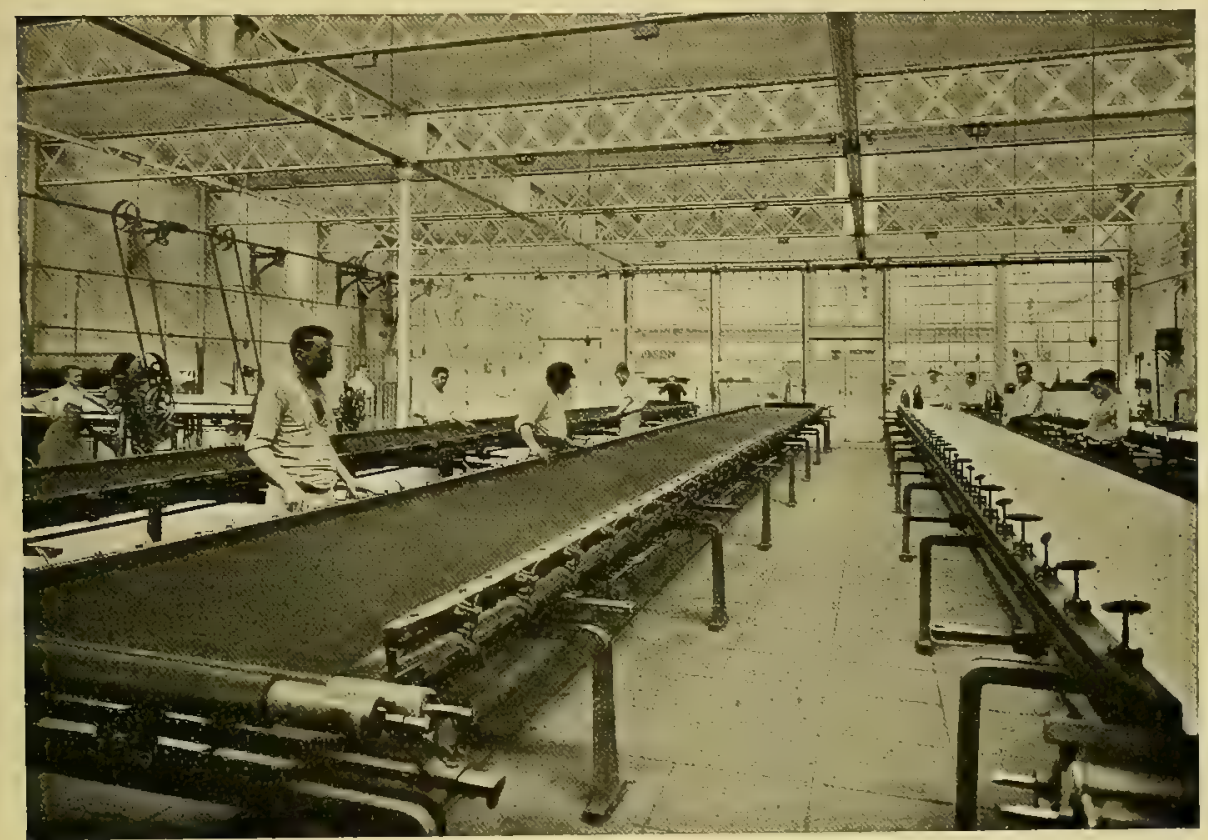

ATELIER D'APPRÊT DE SOIERIES '.

modent également bien d'un apprêt donné de certaine façon, assez légèrement pour qu'il soit peu reconnaissable et dissimulé, et n'enlève rien au soyeux de l'étoffe, tout en augmentant son lustre, son épaisseur et presque sa solidité.

Le principe de l'apprêt est assez simple; il consiste à imprégner d'abord le tissu, parfois à l'envers seulement, quand il en présente un, ou entièrement même, par immersion complète dans un bain d'une dissolution dans l'eau de substances gommeuses, comme les gommes arabiques, la gomme de Sénégal, la colle de poisson, l'amidon, la dextrine, etc., etc., en proportions et en mélanges que l'expérience indique. Il faut s'arranger pour que le tissu, une fois mouillé, reste humecté du liquide autant qu'il convient, et sans excès.

On le dispose alors, on le maintient tendu horizontalement sur une certaine longueur - l'opération se faisant en général à plusieurs reprises -

I M. Henry Bertrand, Lyon. 
sur des sortes de métiers dit rames, où il prend la largeur voulue, et on le sèche par la chaleur directe d'un foyer promené au-dessous, ou par l'air chaud ou simplement abondamment renouvelé par une ventilation énergique. Ou bien encore ce séchage est opéré en faisant passer l'étoffe humide sur un cylindre ou tambour chauffé intérieurement, etc., etc.

Car ce problème simple de l'apprêt manié, remanié, retourné, envisagé de toutes les manières et à tous les points de vue, a donné naissance à la quantité des solutions industrielles dont on voit l'application dans les usines actuellement occupées à ces manipulations diverses avec un développement croissant.

Pour avoir, en effet, une idée de cette importance, il faut s'imaginer ce que représente en kilomètres le chiffre total annuel ou même mensuel des Mousselines de Soie, par exemple, qui sortent apprêtées des organisations spéciales existant pour cet article. Il est, certes, plus qu'imposant, en même temps que très grande est la complexité des apprêts variés et gradués, car il $y$ en a et il en faut pour tous les goûts, tous les emplois et toutes les consommations : apprêt ferme, demi-ferme, souple ou dérompu, chiffon, tendu, créponné, etc., etc.

Il ne faut donc pas songer a entrer ici dans les détails de ces manipulations qui se ramifient et se multiplient avec l'invention et l'ingéniosité de ceux chargés de leur exécution. C'est ainsi qu'ont pris naissance quantité de moyens de perfectionnement du tissu de soie, parmi lesquels citons : le $\operatorname{cr} \hat{\mathcal{C}}-$ page, manière de donner à l'étoffe cet aspect spécial brouillé et ondulé du crêpe qu'on peut obtenir, comme nous verrons, également, par d'autres moyens; le grillagc ou flambage, opération destinée à enlever le duvet d'un tissu en général mélangé, en le faisant circuler rapidement à travers une flamme de gaz réglée en conséquence, les opérations spéciales au Velours sur lesquelles nous reviendrons à propos de ce tissu; enfin, des traitements variés qui modifient si franchement l'aspect et le caractère des étoffes auxquelles on les applique, qu'ils se classent plus logiquement parmi les moyens de décoration du tissu de soie.

Tel est le gaufrage qui fait apparaître en relief sur le tissu des dessins ou des effets particuliers par le moyen de rouleaux de cuivre gravés ad hoc en relief, chauffés et contre lesquels ce tissu passe en subissant la pression convenable, procédé qui s'applique également, comme on sait, aux cotonnades, au papier, au cuir, etc.

Le plissage, par lequel des tissus de soie comme les Satins, les Crêpes de Chine et surtout les Mousselines reçoivent et gardent de nombreux plis petits ou grands, réguliers ou irréguliers, dépend directement du gaufrage et s'exécute par des moyens variés.

Le moirage est beaucoup plus spécial à la soierie; nous parlerons de cette manipulation à l'étude des Taffetas, Failles, etc., étoffes à grain qui se prêtent si particulièrement à son application.

Le perforage est un procédé, d'application beaucoup plus restreinte, qui a servi parfois à orner des Taffetas par des séries de trous ou jours disposés d'après un dessin et pratiqués au moyen d'aiguilles de cuivre poli fixées sur une plaque, qui traversent le tissu et qui laissent quand on les retire une ajouration que 1'on fixe par la chaleur et un certain apprêt. 
Enfin, l'impression est le type de ces manipulations qui ajoutent essentiellement au décor de l'étoffe de Soie pour l'enrichir parfois jusqu'à un degré très élevé ; et la description de ses procédés trouvera au cours de ce travail le développement spécial qu'ils méritent en raison de leur importance actuelle.

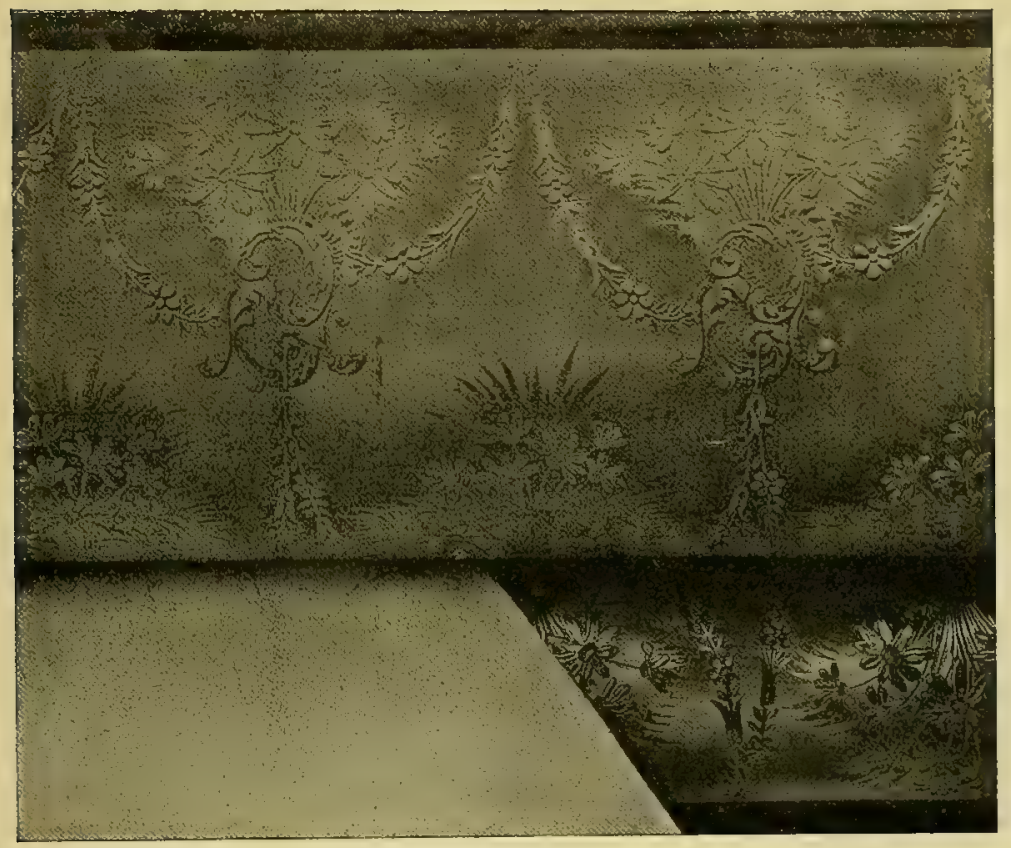

Rouleaux servant au Gaufrage des étoffes de sole. 


\section{COMMENT DENOMMER ET CLASSER LES SOIERIES ?}

Il faut l'avouer, rien n'est plus sujet à erreurs, contradictions, incertitudes et perplexités que l'appellation des tissus de soie, chose extrêmement variable en réalité selon les temps, les lieux, les goûts et les habitudes. On voudrait pouvoir toujours disposer à cet usage de termes précis, impliquant en eux-mêmes définition pour nommer ces étoffes; certes, il en existe au moins un certain nombre employés régulièrement par les gens de métier, mais dans la pratique on se heurte trop fréquemment à des déformations de sens que l'usage a causées, à des conventions ou à des approximations à peu près obligées. Et cela explique pourquoi, si souvent, on appelle par exemple Broché un Damas qui ne l'est pas, Brocatelle ce qui est Lampas, ou toute autre chose, et le reste à l'avenant.

A quelle autorité pourrait-on s'en référer pour plus d'ordre et de netteté? Ce n'est pas en tout cas à la tradition. On sait l'exemple de la pourpre des anciens et toutes les opinions différentes qui ont été émises, sans finalement qu'on soit arrivé à se mettre d'accord sur ce que pouvait être cette nuance par rapport à celles que nous connaissons, ou encore l'étoffe qui en était teinte, car le mot pourpre a désigné, paraît-il, en beaucoup de cas, le tissu lui-même: certains textes parlent même de pourpre blanche ou noire! Complète incertitude ou à peu près également pour les soieries de l'époque médiévale et leur correspondance avec les nôtres. Chansons de gestes, chroniques, poèmes ou proses nous ont transmis des noms auxquels, par simple probabilité la plupart du temps, nous essayons de faire correspondre nos tissus actuels sans affirmation certaine permise.

Qu'étaient-ce exactement que le Paile de soie, le Cenda1, le Samit, le Baudequin, le Camelot, le Siglaton, le Diapre, l'Escarimant, etc., etc.? On peut le conjecturer ingénieusement, en s'aidant de recherches patientes, de rapprochements de textes, et c'est tout.

On est beaucoup mieux fixé évidemment sur les soies moins vieilles que plus tard, sous les règnes de Louis XIV, Louis XV et Louis XVI, on décora de noms tels que: Gros de Tours, Velours de Gênes, Damas de Florence, Dauphines, Persiennes, Musulmanes, Prussiennes, Cachemiriennes, Peaux de Poule, Velours à parterre, Velours mignatures, etc., patronages inspirés par les provenances, les circonstances, certaines imitations et toutes sortes de choses, somme toute, fort étrangères à la fabrication proprement dite et à la composition du tissu.

Et cette manière de procéder ayant persisté depuis lors, étant arrivée mème à tout ce que la fantaisie la plus prononcée peut aujourd'hui suggérer à ceux ou celles dont c'est l'affaire, nous connaissons les noms ronflants et fréquemment renouvelés que, sous l'empire de l'actualité, on applique à telles ou telles soieries pour en pimenter la nouveauté, qui n'est souvent, à vrai dire, qu'une sorte de recommencement. 
Impossible donc de fonder une base autrement qu'illusoire dans cet ordre d'idées de la nomenclature, pour établir une classification et une précision que rien n'impose du reste; mieux vaut s'en tenir à des catégories largement et généralement envisagées et qui peuvent se comprendre à plusieurs points de vue.

En premier lieu, une distinction toute évidente est celle des deux grandes catégories : soieries unies, « en plain» ou « en plein » comme on les appela longtemps, et soieries faconnées ou à dessins; il conviendrait d'y ajouter une division intermédiaire, soieries fantaisies, comme celles à rayures, à quadrillages, etc., qui n'ont pas l'aspect tout uni et ne sont pas cependant fabriquées avec les métiers de façonnés.

Nos modernes Taffetas, Satins, Velours, Mousselines, Gazes, Crêpes, connus de tous, forment une majorité dans les unis, mais y sont compris également nombre d'autres tissus sans dessins, dont la contexture est variée et que l'on désigne habituellement par le terme Armure, pris ici comme la résultante tissée du mode de croisement donné aux fils sur le métier, qui est aussi désigné, et en premier lieu, comme nous l'avons vu, par le mème mot.

Les spécialistes distinguent les armures simples ou fondamentales: Taffetas, Sergé, Satin, Reps, Cannelé, avec leurs dérivés, ce qui fait rentrer, par exemple, une foule de tissus, en apparence très divers, dans la catégorie Taffetas; et les armures composées, celles-ci étant un emploi combiné des premières entre elles pour obtenir des effets différents. Quoique ce champ ait été considérablement exploité - il suffit, pour le voir, de se reporter à un quelconque traité technique - il reste ouvert à l'initiative des chercheurs. C'est par la disposition variée des organes du métier, commandant le jeu différent des fils de chaîne, par la grosseur, le nombre, la proportion, la torsion de ceux-ci, par l'emploi de trames étudiées que l'on parvient à ces créations. C'est bien, en effet, un travail de création, selon le terme usité, que cette recherche, où, théoriquement d'abord, la plume et le pinceau à la main, on trace sur le papier des ébauches et des projets calculés pour passer ensuite à l'exécution pratique; le problème est souvent difficile à solutionner; il exige un savoir patient et entraîné, d'autant qu'il est limité par les données du métier lui-même.

Chose curieuse : il arrive qu'au cours de ces essais et de ces tâtonnements de l'échantillonnage, il se glisse une erreur fortuite, un oubli, une négligence; le travail du métier ne s'exécute pas comme on l'avait imaginé et prescrit à l'avance; or, le résultat incorrect peut se traduire quelquefois par une armure imprévue qui fait nouveauté, a du succès et parfois beaucoup de succès. On pourrait citer des exemples remarquables de ce fait.

Nous verrons, en parlant à leur place des soieries à dessins, celles que dans le public on classe volontiers d'ensemble sous le nom de soies brochées, quelle ampleur et quelle complication peuvent atteindre la recherche et l'établissement de tissus nouveaux, quand il faut combiner à la fois le dessin dans ses détails imposés et le tissu dans son entente, sa qualité et ses couleurs, en laissant le moins possible au hasard et s'aidant pour cela de points de repère connus et toujours du calcul.

Une chose à prévoir spécialement d'une façon générale, c'est le prix et des bases de comparaison permettant de frapper assez juste. C'est une 
question d'habitude et d'entraînement professionnel, aussi bien que pour un travail analytique non moins méticuleux qui est celui de la décomposition. Il consiste, en partant d'un échantillon d'un tissu de soie quelconque, mème presque minuscule, à en discerner, loupe en mains, les éléments aussi bien que la façon dont ils sont assemblés et leur proportion, puis à parvenir par le calcul et l'expérience à la reconstitution aussi exacte que possible de ce tissu pour le fabriquer à nouveau, tout pareil.

La destination des soieries pour tels ou tels emplois permet aussi de les répartir en différents genres, comme: les soieries pour robes et, daṇs cette classe, notons, comme l'on dit, le courant, les tissus de fond, la fantaisie et la nouveauté, celles-ci activement soumises à l'empire de la mode renouvelée aux saisons d'hiver et d'été; les soieries pour la Mode ou garniture des chapeaux - type les rubans - pour les doublures, la cravate ou le $\mathrm{col}$; - les soieries pour l'ameublement, compartiment incontestablement le plus riche en traditions décoratives, etc., etc.

Toute une branche commerciale repose sur l'étoffe de soie destinée au parapluie et la place prise par cet accessoire dans les usages du monde entier donne à penser quelle peut en être l'importance.

Enfin, on pourrait encore différencier les soieries actuelles par leur composition, car souvent elles ne sont plus tout soie, mais mélangées avec d'autres textiles: laines, cotons, soie artificielle, schappe, lin, ramie, sans parler du métal : or et argent.

Rappelons également encore une fois, et pour mémoire, cette distinction primordiale qui s'impose aujourd'hui et vient de la façon d'appliquer la teinture donnée, soit avant le tissage aux fils mêmes pour le teint en fils, soit après le tissage aux pièces ou teint en pièces, et concluons que, dans l'impossibilité de prétendre à une rigueur méthodique dont on ne voit pas la nécessité et dans un ordre tout arbitraire si l'on veut, mais se rapprochant de la marche habituelle du simple au composé, nous nous proposons maintenant de passer en revue les principaux types d'étoffes de soie en signalant de notre mieux les caractères qui les distinguent et les particularités qui se rattachent à chacun d'eux. 


\section{LE TAFFETAS}

A tout seigneur, tout honneur. Or, 1e Taffetas ${ }^{1}$, la plus simple, comme nous l'avons vu, et la plus répandue des armures dans le domaine textile, a régné et règne encore à ce titre en maître dans le département de la soie, car si ce mode élémentaire du croisement des fils de la chaîne est, sans aucun doute, historiquement le plus ancien, il est aussi, en bien des cas, le meilleur, comme l'usage l'a démontré, pratiquement, pour les tissus de soie. Le taffetas, ou, en langage canut, le système de l'armure par « une prise, une sautée » (sous-entendu lisse), a donné lieu à mille variantes et doit être considéré comme le pivot sur lequel repose tout l'art du tissage soyeux.

Car c'est aujourd'hui une famille innombrable de tissus qui se réclame de cette paternité; il y a toujours des variétés de taffetas, étoffes serrées, fournies, résistantes, qui sont la suite des taftas ou taphetas, de toutes couleurs, vermeil, armoisin, zinzolin, vert, violet, etc., comme de toutes provenances, de Florence, de Bologne, d'Avignon, de Tours et de Lyon, dont

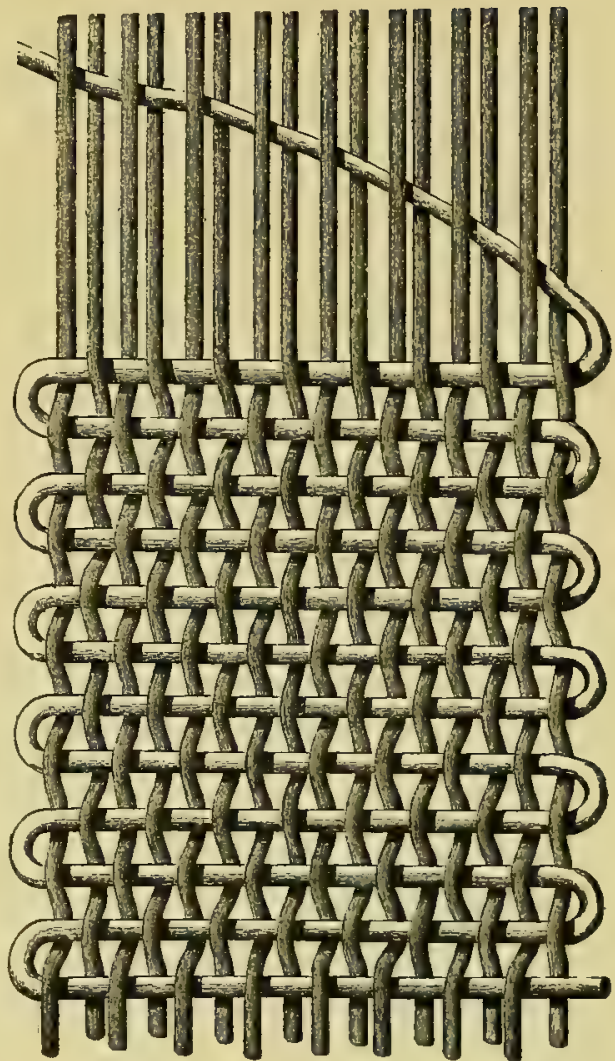

SchÉma du TAFFetas. les dames et gentilshommes d'antan aimaient à se vêtir ou à parer leurs demeures; déjà en I 389 , le taffetas était si commun que l'on en put tendre tout un pont à l'entrée d'Isabeau de Bavière à Paris ! Mais, au premier abord, comment songer à rattacher au genre taffetas des tissus si différents de son aspect classique, tels que les légères et aériennes Mousselines de Soie, les Crêpes vaporeux, les Foulards, les Pongées, les Tussors exotiques et bien d'autres encore, oủ le jeu des fils est régi, cependant, par la même loi simple et identique?

Ainsi, la dénomination peut varier selon la quantité de chaine ou de trame employée et leur proportion respective et encore selon les prove-

1 Peut-être faut-il voir l'étymologie de ce mot dans l'onomatopée: taf-taf, qui exprimerait le bruit caractéristique que produit cette étoffe quand on l'agite! 
nances, mais tout tissu fabriqué d'après le croisement du taffetas sera toujours du taffetas.

Le caractère le plus marquant, le plus général des taffetas habituellement connus sous ce nom, est de présenter sur ses deux faces pareilles, sans envers ni endroit, un aspect assez lisse et tendu avec, cependant, un grain résultant des côtes fines ou fortes que forment les fils de la trame en se juxtaposant les uns à côté des autres perpendiculairement aux fils de chaîne qui les enserrent. On voit très bien ces côtes à l'œil ou à la loupe, et on les sent parfaitement au toucher, procédé d’appréciation et presque de mesure qui joue un grand rôle dans la fabrication comme dans les transactions, et qui, développé par la pratique professionnelle, donne des renseignements très utiles.

Le grain, perceptible dejà dans les taffetas dits fonds de jupe ou « petite soie $\#$, s'accuse, se marque avec la qualité, devient très apparent dans la faillc, qui eut une si grande vogue sous le Second Empire : c'est un taffetas dont la trame est bien plus grossie et renforcée que la chaîne, tout en étant rendue moins brillante que celle-ci par la manière de la teindre. Il en est de même avec des variations de grosseur, parfois de régularité, dans ces genres que l'on ne connaît plus guère actuellement que par le souvenir : pou ou poult de soie, et la catégorie des gros grains, gros de Naples, gros des Indes, gros de Londres, etc., à laquelle il conviendrait d'ajouter les genres dits taffetas cannclés, velours ottoman, faille francaise, etc.

L'appellation gros de Tours, qui a correspondu longtemps à de beaux taffetas fabriqués dans cette ville à limitation de ceux de Naples, très connus aussi par les tapissiers sous le nom qui s'est conservé de Quinze-Seize (de sá largeur $15 / 16^{\circ}$ d'aune), s'applique également à un taffetas dont la trame est doublée, ayant été passée par deux coups successifs sous la même levée des fils de la chaine. Cette combinaison n'est employée aujourd'hui, à vrai dire, que pour les soieries à bandes ou à dessins, en mélange et en opposition particulièrement heureuse avec le satin par exemple.

La Louisine, au contraire, est un taffetas dans lequel ce sont les fils de la chaîne qui, doublés, marchent deux à deux. De la sorte, cette armure sépare, ouvre davantage les fils et donne un aspect plus granité à l'étoffe, un toucher plus souple; tandis que l'habituelle tenue du taffetas lui donne cette carte ferme que les emplois de la mode tendent à proscrire pour le moment et qui le fait se casser en plis assez raides et anguleux, bien que les reflets de cette étoffe restent soyeux et agréables.

Une façon fort ancienne de varier l'aspect du taffetas uni est de rendre celui-cichangeant ou glacé, ce qui s'obtient en tramant une chaîne d'une couleur avec une trame d'une nuance opposée; ne ferait-on pas remonter cet usage plus haut qu'à l'année $\mathbf{3 2 8}$, où, d'après un inventaire du temps, le taffetas changeant coûtait alors I 7 sols l'aune? On imagine facilement la variété considérable de coloris que, depuis lors, la recherche constante a permis d'atteindre en combinant ces nuances de changeants d'après la fantaisie et le goût de chacun pour obtenir souvent des effets fort agréables à l'œeil. Après les changeants ou glacés à deux nuances, on est même arrivé au mélange de trois couleurs et au taffetas dit caméléon, en employant, avec une chaîne d'une première teinte, une trame composée elle-même de fils de deux couleurs différentes, assemblés dans.la navette. 


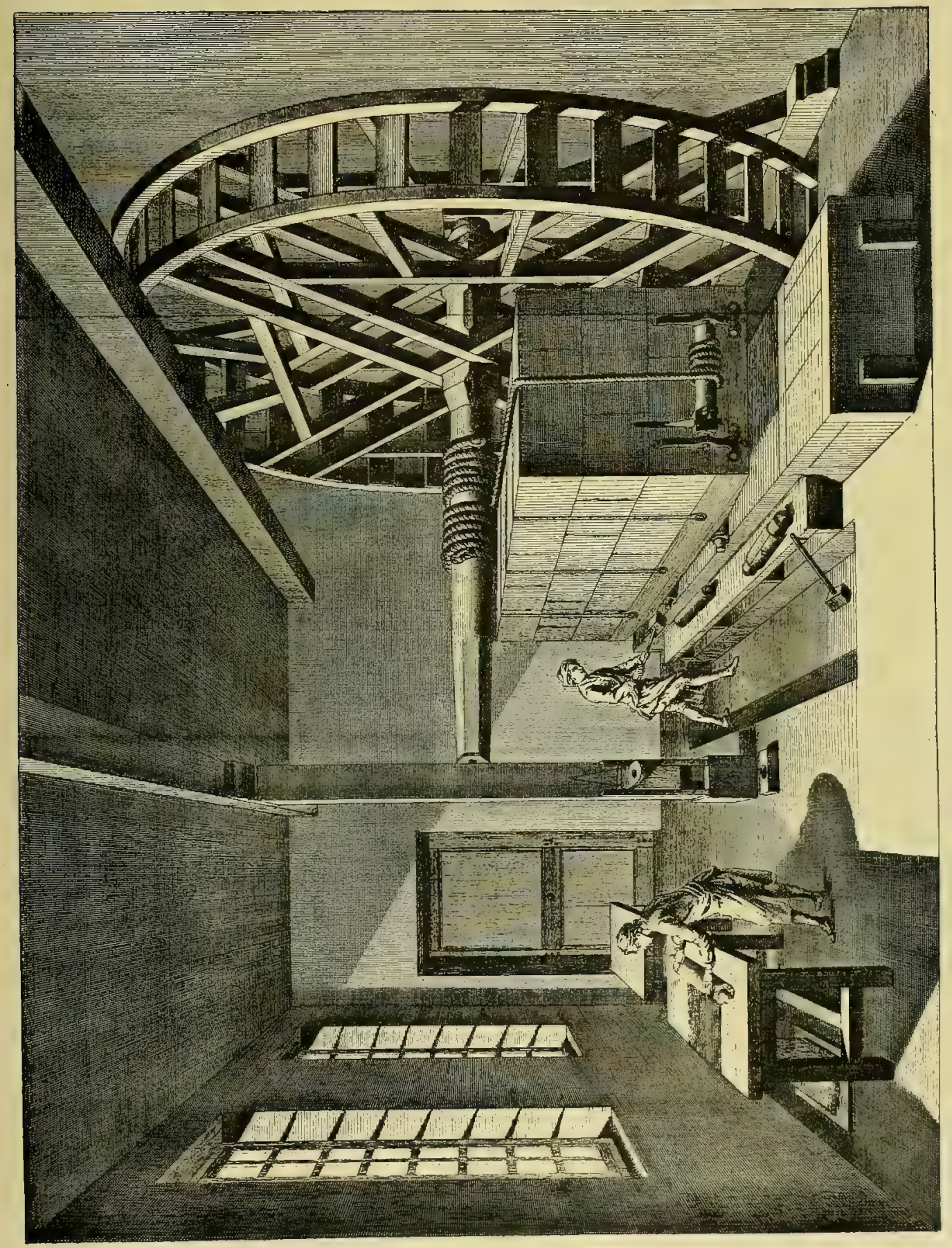

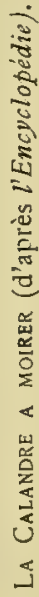


C'est encore du taffetas que se réclament les foulards et les toiles de soie, tissus oủ la légèreté, la souplesse, la minceur sont des qualités d'abord requises aussi bien qu'une solidité assez grande, toutes proportions gardées.

On disait autrefois de pareils tissus, de façon plus pittoresque que très exacte, que c'était « une manière de petite étoffe très claire, fort légère, et " point croisée, faite sur le métier avec de la soie filée, dont les femmes se « servent à faire des fichus, ou mouchoirs de cou, ou autres hardes semblables». Mais on les fabriquait alors avec des fils teints, tandis que cette variété de tissus dont l'emploi est considérable est entièrement traitée maintenant par la teinture en pièces. On en fait aussi bien des robes que toutes sortes de choses élégantes et pratiques, et les pays exotiques, Chine ou Japon, qui avaient largement devancé le Vieux Monde pour cette fabrication particulière, sont restés de grands fournisseurs de ces étoffes englobées sous le nom très général de Pongées; ils arrivent en Europe en écru ou simplement avec une première préparation et sans teinture; on les teint, les apprête et les finit à la manière occidentale.

Taffetas, failles, gros de Tours et poult de soie se prêtent tout spécialement bien à une opération que nous avons mentionnée plus haut, celle du moirage, dont la technique resta pratiquement inconnue en France jusqu'au milieu du $\mathrm{XVII}^{\mathrm{c}}$ siècle, moment où elle fut introduite à Lyon par 1'Anglais Badjer ou Badger qui vint s'y fixer vers I754. Auparavant, la moire ou moëre, comme on l'écrivait alors (de mohair, paraît-il, par analogie avec le poil de chèvre très brillant ainsi dénommé), livrée à la consommation, était jusqualors de production exclusivement anglaise, s'il paraît démontré toutefois que le principe de cette opération fût originaire de la Chine.

Badjer, dit Joubert de l'Hiberderie dans son curieux livre le Dessinateur pour les Fabriques de Soie, «nous a apporté d'Angleterre le cylindre merveilleux, et l'apprêt avec lequel il fait d'un gros de Tours une véritable glace ».

En effet, le moirage donne à l'étoffe à laquelle on l'applique comme le reflet d'une glace ou plutôt il lui communique un aspect comparable à la surface diune eau transparente et profonde, sur laquelle courent des rides ou des ondulations dites filets - de la moire, imitant les remous et les agitations légères. Ces effets si typiques et imprévus, qui ont séduit et retenu bien souvent l'imagination des poètes et des artistes, ne sont autres que des jeux prosaïques de la lumière réfléchie, réfractée inégalement, curieusement, sur la surface du tissu; il faut, pour cela, que cette surface ait été modifiée, que son grain ait été écrasé pa places (on voit ici la nécessité de traiter des étoffes à grain ou à côtes), par la pression considérable qu'on fait subir à l'étoffe ; c'était autrefois avec la lourde calandre de pierre et maintenant c'est avec la moderne presse hydraulique; en même temps, on a ménagé certains dérangements, certains glissements de l'étoffe repliée, comme il convient, sur ellemême. La moire de Badjer ou moire antique s'obtenait et s'obtient encore en repliant forcément la pièce à moirer par son milieu et lisières sur lisières avec de grands soins, et la conséquence forcée est un pli ineffaçable, on le conçoit, au milieu de l'étoffe, pli que l'on s'ingénia à dissimuler par des artifices et des arrangements et aussi par exemple, en certains cas, en fabriquant deux pièces identiques tissées l'une à côté de l'autre, en jumelles, par conséquent 
de tissage identique, pour les moirer ensuite en les appliquant l'une sur l'autre.

Vaucanson porta son attention sur le problème mécanique de la moire entre mille autres et imagina la calandre cylindrique, sorte de laminoir à rouleaux entre lesquels l'étoffe passe en subissant la pression nécessaire. Mais cette machine, qui simplifiait le système ancien, ne réussit pas à le remplacer, car son emploi permettait de produire seulement la moire ronde, dite plus tard moire française, qui présente des effets beaucoup moins variés et imprévus que la moire antique. Dans la moire ronde, en effet, que l'on obtient encore aujourd'hui par un système tout analogue, on

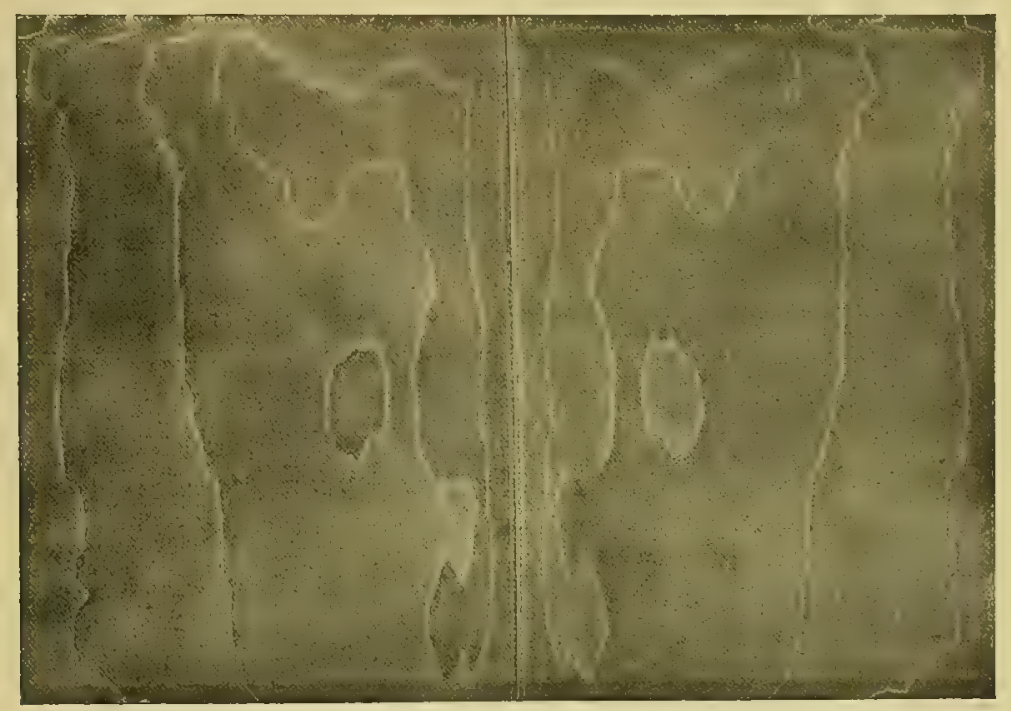

MoIre antLue.

retrouve constamment des formes arrondies, presque régulières, qui imitent assez bien les contours veinés que l'on remarque sur une coupe de bois de chêne ou de pin.

Actuellement, avec l'incessant besoin de nouveauté, on est arrivé à produire de nombreux genres de moire par divers procédés qui se perfectionnent et se multiplient chaque jour; par exemple, on peut très bien moirer le tissu par places ou par bandes et l'on parvient à modeler les effets de la moire ou à les remplacer par de grandes ondes d'un bel aspect lumineux et décoratif, ou bien encore à former des sortes de dessins imprécis et curieux, etc., etc.

D'ailleurs, tout comme au XVIII ${ }^{e}$ siècle et d'après les mêmes errements appliqués par Badjer et ses contemporains, on peut user de la moire en mélange avec des dessins produits sur l'étoffe par le métier pour décorer certaines soieries à fond gros de Tours de la façon qui eut un si gros succès à cette époque.

Chose digne de remarque : on trouve fréquemment mentionné au moyen âge, et même plus tard, un tissu de soie appelé Tabis, nom dérivé des formes 
orientales et arabes, atabis ou zatabis, et auquel est constamment attribuée la caractéristique d'être d'un aspect ondé ou ondoyant. Les tabis, sortes de gros taffetas dont l'usage s'est perdu, étaient peut-être bien déjà moirés, tabisés par le moyen de la calandre, et d'autres conjectures font supposer qu'ils pouvaient aussi comprendre une chaîne de soie et une grosse trame de coton ondée par sa torsion particulière.

Avant de passer outre, en nous proposant de revenir, à une place spécialement réservée, sur les mousselines, crêpes et autres tissus légers du même genre, disons qu'il y a des taffetas où la trame soie est remplacée par une trame de laine ou de coton; c'est la catégorie des Bengalines, Eoliennes, Popclines, Taffetalines, etc., à destination de divers usages, tandis que d'autres taffetas encore, dénommés par exemple Marcelines, Florences, ceux-ci fort minces et légers, quoique tout de soie, s'appliquent à peu près uniquement aux emplois de la doublure.

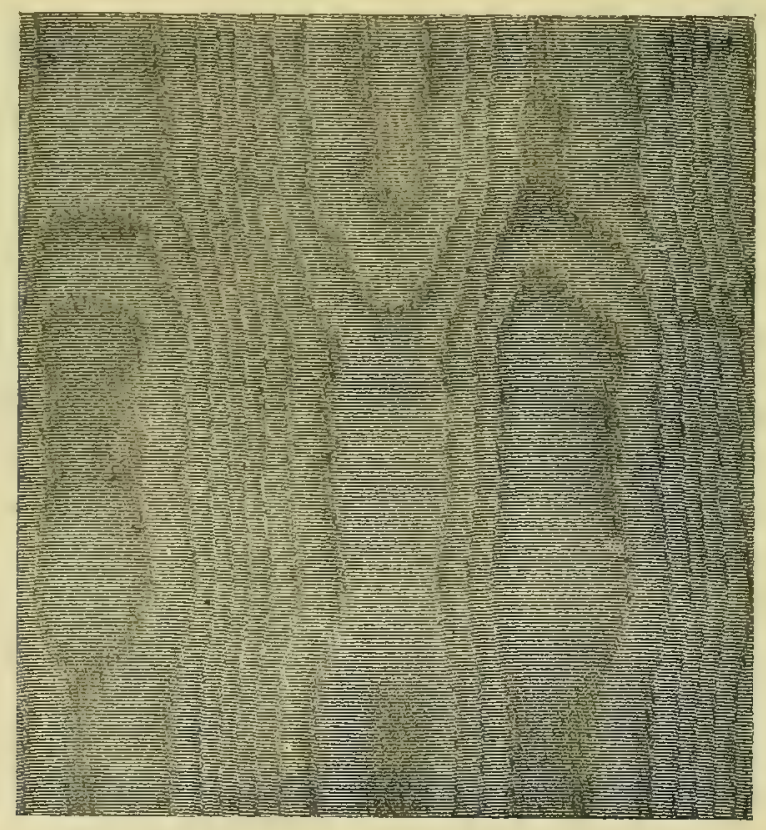

MOIRE FRANÇAISE. 


\section{LE SERGÉ}

Tout le monde connaît la Serge ou Diagonale, étoffe de laine, caractérisée par les côtes obliques formées à sa surface, de façon plus ou moins apparente, et qui se prolongent d'une lisière à l'autre. Or, ce nom de Serge, dont l'origine n'a pas été bien précisée (quelques-uns le font dériver de l'espagnol : Xerga), ne s'applique certainement pas à la matière même dont est faite l'étoffe et à sa nature; il en désigne plutôt la contexture, que l'on retrouve identique dans maintes soieries, dites Sergés, comme les Surahs, les Twills, les Sergés pour parapluies, etc.

Cette manière de faire croiser les fils dans le sergé, cette armure, présente avec le taffetas des différences notables; ainsi, sauf en quelques cas, - les fils de la chaîne ne se partagent plus en deux moitiés pareilles, entre lesquelles la trame vient prendre place, mais, au contraire, par tiers, parquart, ou dans une proportion variable et fractionnaire, d'après les mêmes principes d'armure.

Il est ce principe, que la figure

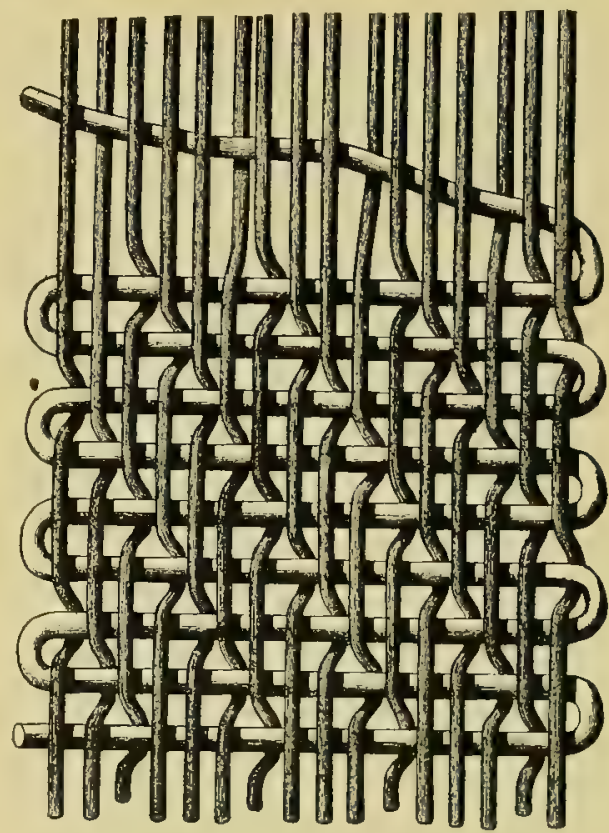

Schéma du Sergé. aidera mieux à comprendre, de faire mouvoir les fils de la chaîne par la manœuvre des lisses, leur levée, dans un ordre et une suite tels que ces fils forment successivement des sortes de points très fins, des flottés, qui se disposent les uns à côté des autres, avec une dégradation, un décochement presque insensible, mais très régulièrement pour produire par leur juxtaposition ces sillons en diagonale, ces côtes typiques du sergé. Dans l'exemple choisi, qui est celui obtenu sur quatre lisses - où, comme on dit, sergé de 3 lie I — chaque fil flotte ou reste du même côté de la trame, pendant trois coups passés de celle-ci et lie ou passe de l'autre côté, au quatrième coup; le fil voisin fait de même, avec un point de départ qui diffère d'un coup de trame, et ainsi de suite pour les autres. En raison du grossissement très fort de la figure, on distingue ce jeu des fils qui produit, on le conçoit, un envers et un endroit sur le tissu, car le sillon formé par la chaîne sur une des faces détermine sur l'autre un sillon analogue, mais d'obliquité contraire, résultat des points ou flottés de la trame. C'est le cóté des effets de chaîne que l'on considère le plus généralement comme l'endroit. 
Dans un sergé fin ou court de rapport, l'effet de diagonale paraît souvent fort peu, mais on le rend beaucoup plus marqué en augmentant la longueur des points ou flottés par l'armure, ce qui nécessite l'emploi d'un plus grand nombre de lisses au métier, par exemple jusqu'à Io ou I 2 pour des sergés de 9 lie I ou I lie I, avec un maximum que l'expérience a déterminé.

Les côtes obtenues de la sorte sont très apparentes, mais c'est forcément un peu aux dépens de la solidité du tissu, auquel les fils, moins liés entre eux, donnent moins de tenue et de résistance aux frottements, et qui glisse quelquefois trop facilement sous le doigt.

Il est possible d'ailleurs de parer à cet inconvénient dans une certaine mesure, en s'arrangeant pour avoir entre chaque flotté un peu grand plusieurs points serrés oủ le fil de chaîne est lié par la trame en taffetas; on réalise ainsi des sergés, dits composés, oủ les côtes larges sont encadrées de parties qui les maintiennent dans le bon ordre. On peut aussi faire varier la grosseur des côtes entre elles, en intercaler de plus fines entre de plus grosses, faire différer l'espace qui les sépare, leur donner diverses directions, en former des lignes brisées quion nomme chevrons, des sortes de tout petits carreaux, des losanges, des granités, etc. C'est, en un mot, user de toutes les ressources des dérivés du sergé, dont on s'est ingénié depuis longtemps à multiplier le nombre et qui, sous les dénominations les plus diverses, forment une classe importante de soieries dont certaines, à leur heure, connurent le plus vif succès.

Un sergé remarquable est celui qui résulte du croisement par moitié des fils de la chaine, comme dans le taffetas, et qu'on nomme aussi croisé ou Batavia ou encore 2 lie 2; cet énoncé théorique le définit parfaitement, si l'on s'en rapporte aux explications ci-dessus : chaque fil forme des points ou flottés, régulièrement de deux coups de trames de chaque côté du tissu; 1es côtes se produisent de façon toute semblable sur chaque face et il n'y a ni envers ni endroit.

Ce batavia est solide et souple, et des armures analogues ont été très

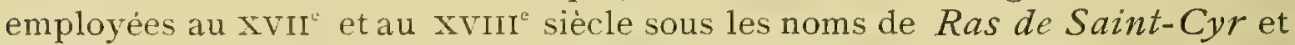
Ras de Saint-Maur. Les ras de Saint-Maur étaient les plus estimés et se faisaient en tout noir pour habits de petit deuil ou de cérémonie.

La Lévantine, sergé de quatre, est un tissu bien oublié aujourd'hui. Le Surah, nom d'usage plus récent et qui ne correspond pas à telle ou telle armure définie, englobe des sergés dont l'emploi est subordonné à la mode; le surah glacé, tout comme les taffetas de ce genre, est fait d'une chaîne d'une couleur et d'une trame d'une autre couleur opposée. On a nommé et on appelle encore Polonaises des sergés divers qui, tramés coton et teints en pièces, sont destinés à la doublure, tandis que les Silésiennes, Austrias, etc., sont des sergés tramés laine, employés pour les parapluies.

Twill est le nom anglais qui désigne les sergés en général, et plus particulièrement peut-être ceux d'importation exotique, japonaise surtout, qui sont classés d'habitude avec les foulards et les pongées dont nous avons parlé.

Enfin, le plus simple des sergés dans son principe, sergé de 2 lie I, a connu et connaît encore sous divers noms, dont un très répandu, Satin de Lyon, et avec diverses adaptations modernes, une telle notoriété que son étude paraît devoir figurer en meilleure place avec celle de la vaste catégorie des satins que nous allons aborder sans plus tarder. 


\section{LE SATIN}

Les mots satin, satiné, sont si répandus qu'il est presque superflu de rappeler la signification qu'on leur accorde et qui procède, sans aucun doute, des caractères si connus de l'étoffe soyeuse de ce nom; le satin, en effet, possède le brillant, le poli et cette douceur au toucher qui, par comparaison, ont doté la langue d'une épithète et d'expressions consacrées par l'usage.

L'opinion étymologique qui fait dériver satin de l'italien setino, de seta, soie, confirme en effet que ce tissu était dès l'origine, comme il l'est encore souvent, celui dans lequel la soie peut déployer le plus complètement peut-être les qualités qui lui appartiennent en propre et joindre un éclat miroitant sans rival à une moelleuse et riche épaisseur.

Synonyme de beauté, d'opulence, le satin le fut encore parfois de " gaîté 》, car nos ancêtres affectionnaient tellement cette étoffe que, voulant caractériser quelqu'un de très gai, ils le comparaient au satin ${ }^{1}$. Dès qu'il fut connu en Occident, le satin

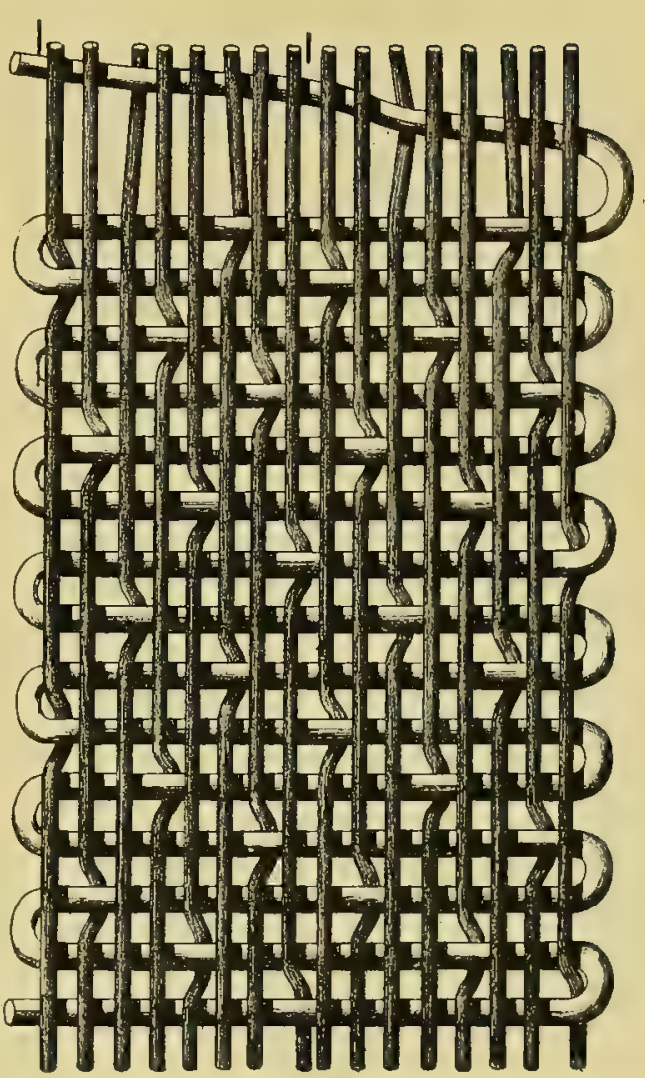

SCHÉma dU SATIN.

y occupa une des toutes premières places dans la hiérarchie des soieries. Au $\mathrm{XVI}^{\circ}$ siècle, il était réputé supérieur au damas, déchu de son rang primitif, et si, dans les cérémonies oú figuraient les Parlements, un Président était habillé de velours, les Conseillers l'étaient de satin,et le damas était laissé aux greffiers comme le taffetas aux huissiers.

Et les satins cramoisis, ou incarnadins de Venise, qui coutaient alors en France 2 écus $2 / 3$ l'aune, ceux de Gènes, de' Lucques, les satins rouges et violets de Florence et de Bologne, du prix de 2 écus l'aune, figuraient pour une part des plus importantes parmi toutes ces soieries dont il se faisait un si grand trafic en tous pays; ils venaient jusqu'en Flandre où,

I Fr. Michel, Recherihes sur le commerie, la fabrication, l'usage des totfis di Suie alu Moyen lge, t. II, p. 222. 
cependant, à Bruges, par exemple, on fabriquait certains satins qui eurent leur notoriété.

Le satin dont les plis lourds, chatoyants et nacrés ont toujours charmé l'œil et séduit les portraitistes, est bien resté le type de la belle étoffe de soie, mais il a subi les modernes interprétations; elles en ont beaucoup multiplié les variétés et n’ont laissé parfois à certaines que l'apparence très mesurée au poids de la soie de lancienne splendeur de ce tissu.

La liste de ces genres serait fort longue s'il fallait chercher à l'établir très complète depuis les satins de belle qualité, dits Duchesse, employés surtout en blanc et en noir, et longtemps imposés, pour ainsi dire, par le protocole de la toilette, les Satins mervcilleux. Satins de Lyon, d̀ la Reine, Turc, Romuin, etc., etc., de toutes qualités et à toutes fins, jusquaux satins mélangés, tramés de laine, de coton, de schappe, en tombant jusquau très modeste satin à I 9 sous destiné aux plus ordinaires usages; celui-ci est la traduction très revue, très corrigée en style actuel, de ce qui avait été fait dans cette voie dès le moyen àge, sous le nom de demi-satin, et, plus tard, de ce que le canut railleur appela : satin de paurre ou satin d'Albigny ${ }^{1}$.

T'oublions pas un triomphateur récent: le satin Libcrty, qui, né en Angleterre, sous linfluence et les tendances de ce quion a appelé le style. du mème nom, est parrenu à la fortune que l'on sait en faisant le tour du monde; ce fut la victoire dune jeune soierie, apportant toute son inédite souplesse nouvelle, sur l'étoffe à la façon d'autrefois, gardant l'antique soutien assez raide, le ferme maintien un peu engoncé.

On a marché, depuis, à grands pas dans le chemin ouvert aux innovations, où vient de saffirmer le succès d'un autre satin récent : le satin Mćtéor, accompagné de nombreux satellites.

Toutes ces étoffes se distinguent les unes des autres par des différences dans l'aspect, le toucher, l'épaisseur, différences causées, non seulement par le jeu plus ou moins différent des fils, ou par l"armure, mais en grande partie également par le dosage, la proportion de la soie employée, la nature de la trame, dont le rôle très dissimulé dans le satin autorise des mélanges, enfin beaucoup aussi parla façon, soit demployer des fils teints avant tissage, soit de teindre le tissu tout fabriqué, comme dans le cas du Liberty, mais au fond le principe du tissage du satin reste soumis aux mêmes règles, dont il est temps de dire un mot.

Constatons d'abord que, d'après les opinions les plus autorisées, le satin a été apporté en Europe à l'exemple de tous les autres tissus - il nous faudra le répéter à maintes reprises - de l'Inde et de la Chine, pays conservateurs où les procédés de tissage se sont conservés immuables jusqu'à nos jours depuis plus de deux mille ans. Longtemps mème, bien que l'introduction du satin dans nos régions date d'une époque très reculée, les satins chinois et indiens conservèrent une supériorité marquée, que les indiscutables progrès occidentaux firent cesser, sans modifier toutefois ce principe séculaire de larmure satin.

On peut considérer celle-ci comme dérivée de l'armure du sergé; mais, pour obtenir du satin, on règle toutes choses au métier pour que les points,

1 Nom d'une localité voisine de Lyon où se trouve un asile de mendicité. 
les flottés des fils de chaîne, au lieu de se placer en côtes diagonales, se disposent en ordre irrégulier, venant se disposer très serrés les uns contre les autres et tous d'un méme côté du tissu; de la sorte, on obtient une surface plane et brillante, sans grain ni côtes apparentes, qui est l'endroit du satin. Les liages ou points, où les fils de la chaîne croisent nécessairement avec la trame pour former un support évidemment indispensable, se trouvent dissimulés, placés chacun le plus loin possible de son voisin; la chaine, formée de fils choisis en nombre voulu et de grosseur convenable, apparaît avec tout son éclat à l'endroit, qui vaut par elle et cache complètement la trame.

La figure ci-jointe donnera une idée meilleure de ce jeu de fils réalisé par la manceuvre utile des lisses au métier, d’après des règles enseignées par la pratique et avec plus de facilité qu'on ne pourrait le supposer. Il est bon de noter que le travail s'eftectue de telle sorte que l'étoffe est fabriquée endroit en dessous; c'est, du reste, soit dit en passant comme un détail à retenir, la façon dont on procède en tissage toutes les fois qu'on le peut, pour des étoffes à endroit et envers, la pratique ayant démontré pour plusieurs raisons les avantages et la commodité de cette manière d'opérer.

On appelle, dans les milieux de fabrique, satin de cinq, satin de huit, par exemple, les satins obtenus avec ce nombre de lisses respectivement nécessaires; on peut, de même, faire des satins de quatre, de six, de sept, etc., toutes armures beaucoup moins usitées que les deux premières citées, surtout celle faite sur huit lisses, qui est le type par excellence du satin.

Le sergé de trois ( 2 lie I) est, nous l'avons dit plus haut, bien plus connu sous le nom de satin de Lyon. Dans ce cas particulier, en effet, la côte que donne cette armure est si fine et son obliquité est telle qu'elle n'est pas apparente. L'endroit paraît tout à fait satiné, quoique sensiblement plus mat que dans un satin véritable. Un type actuel de ce satin de Lyon est le Météor, dèjà nommé, soierie teinte en pièces et à laquelle l'emploi d'une trame spéciale, très tordue à l'avance, donne une particulière souplesse et le « flou » si goûté.

En combinant un satin de Lyon - teint en fils - avec une trame supplémentairement ajoutée qui se place à l'envers du tissu pour en augmenter l'épaisseur, on a fait la Peau de Soie, qui eut un vif succès il y a quelque vingt ans et $\mathrm{n}$ 'a peut-être pas disparu complètement de la consommation. Le nom qualifie bien cette étoffe d'une matité voulue à l'endroit, acccentuée par la torsion grenadinée, c'est-à-dire augmentée dans une proportion donnée que l'on donne aux fils de la chaîne, d'un toucher épais et tombant et d'une bonne souplesse.

Aux exemples de satins que nous venons de donner, il faut enfin ajouter pour mémoire tous ceux dits " de fantaisie » et "nouveauté » faits en vue des robes, que crée à chaque instant l'imagination des chercheurs en la matière; en beaucoup de cas, on peut dire que ces étoffes sont des rénovations et la mise au jour sous de nouveaux dehors et décorées de noms d'actualité, d'ententes et de combinaisons textiles connues remaniées d'après les moyens dont on dispose aujourd'hui.

$\mathrm{Si}$ le satin uni ne peut se moirer comme le taffetas, du moins il se prête parfaitement au gaufrage, dont nous arons dit quelques mots déjà. 
En certaines qualités, le satin se prète aussi à la combinaison : chaîne d'une couleur, trame de l'autre, pour obtenir des coloris à reflets changeants ou glacés.

L'impression s'exécute on ne peut mieux sur le satin et prête à d'ingénieuses ententes décoratives, entre autres celles du Velours au Sabre, sur lesquelles nous aurons à revenir.

Nais ce qui est à retenir et à remarquer, c'est que le satin s'emploie à merveille en mélange et en opposition avec d'autres armures: taffetas et gros de Tours en premier lieu, pour des étoftes à bandes ou à dessins; le contrastr de sa note brillante, lumineuse, avec des effets plus mats, est particulièrement heureux et ce n'est pas d'hier que l'on a tiré un excellent parti de ces mélanges, comme nous le verrons. 


\section{REPS. CANNELÉ. ARMURES DIVERSES}

Reps : ètoffe de soie très forte, avons-nous lu dans certain dictionnaire, définition qui serait au moins incomplète si elle était exacte par ailleurs. C'est par analogie, sans doute, avec des reps faits de coton ou d'autres textiles que l'on a conclu à la même force et solidité pour le reps de soie, ce qui ne se justifie guère.

Dans le reps, tout à l'inverse du satin, c'est la trame qui joue le rôle important, en formant à l'endroit du tissu un ensemble de points ou flottés

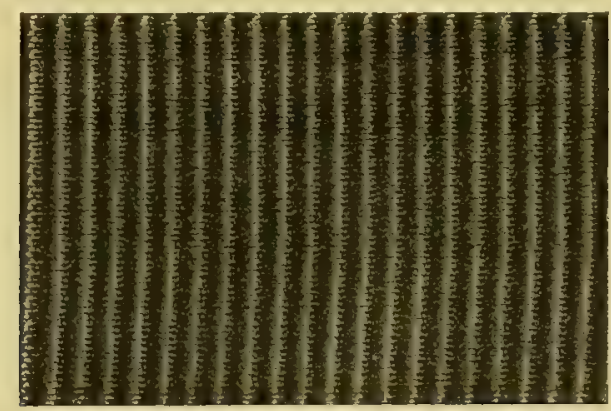

Rers (effet grossi).

qui se superposent en sortes de lignes ou de boyaux dont la direction est celle de la longueur de l’étoffe. Naturellement, pour former un support résistant, cette trame est liée de place en place avec la chaîne, mais dune façon qui reste dissimulée.

L'effet obtenu est curieux; la surface brillante du reps est très régulièrement striée en longueur de fines raies brillantes et juxtaposées; la souplesse du tissu est asse $z$ satisfaisante, mais l'inconvénient est justement de mal résister à l'usage; ces nombreux flottés, ces brides de la trame, dont la longueur peut atteindre plusieurs millimètres et qui sont retenues à leurs seules extrémités, se trouvent très susceptibles d'ètre happées par une aspérité quelconque pour leur plus grand dommage. Le défaut du reps de soie est d'accrocher et, si le fond du tissu résiste suffisamment, l'endroit est rapidement détérioré par les frottements. La fabrication en est assez délicate; elle exige l'emploi d'une trame de soie particulièrement choisie. sans irrégularités ni défauts qui seraient très apparents. Aussi, cettc armure est-elle relativement peu employée pour l'étoffe unie, surtout pour robes, excepté cependant quand la mode en impose la faveur. Mais, par exemple. son mélange avec le satin, le taffetas, etc., dans les genres rayés ou façonnés, donne lieu à des interprétations plus pratiques et plus répandues. 
On en peut dire autant du Cannelé, dont, à vrai dire, le principe est dans le taffetas. En indiquant les variantes et les dérivés de celui-ci, nous n'avons fait que nommer seulement les Gros Grains et les Velours ottomans, sans pouvoir les décrire en détail; mais cependant on aura retenu, sans doute, que l'aspect de ces étoffes de soie est caractérisé par leur grain, autrement dit par les côtes transversales qu'elles présentent dans le sens de leur largeur.

Il en est de même pour les cannelés proprement dits, obtenus par des moyens dans le détail desquels nous n'entrerons pas. Le terme cannelé exprime justement que l'on s'est proposé de réaliser sur le tissu des sortes de cannelures plus ou moins grosses et apparentes. Ces cannelures sont formées par les flottés des fils de la chaîne ou plutôt d'une chaîne ou plutôt d"une chaîne spéciale supplémentaire, dite de poil, de façon à figurer sur un fond de taffetas les lignes brillantes, parallèles, variées de grosseur et

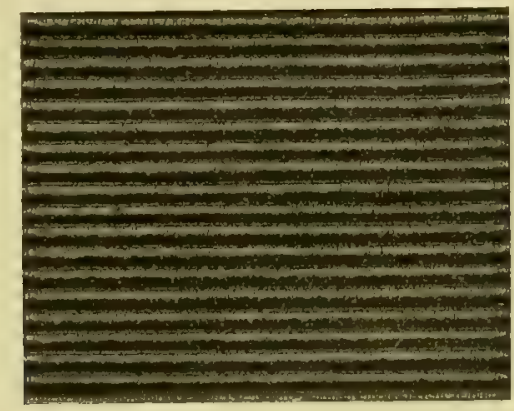

Ċannelé.

d'étendue, dont se composent les différents genres de Cannelés, Cannetillés, Nattés, etc.

A vrai dire, on use surtout de ces armures, et presque uniquement sous la forme de bandes, de filets, de parties isolées, en accompagnement ou illustration de fonds unis, comme le taftetas, sur lequel leur note brillante et régulière se détache très heureusement.

A la fin $\mathrm{du} \mathrm{XVIII}^{\mathrm{e}}$ siècle, on a répété fort souvent cette ornementation, qui, maniée avec le sens de la mesure dans les proportions, comme du goût dans le coloris, a produit les meilleurs et plus délicats modèles de soieries, à bandes ou filets en cannelé; souvent aussi on a combiné ces bandes avec des guirlandes droites ou des montants de fleurettes légères, pour des genres qui furent très recherchés.

Dans le ruban, on a usé pas mal, également, des applications du genre cannelé, disposé en fines lisières et en filetés.

L'habileté technique, l'art des spécialistes a été, possédant les ressources de ces divers jeux des fils, ou armures simples, ou fondamentales, dont nous avons essayé de donner quelque idée, de les combiner entre elles, de les mélanger pour en tirer d'autres plus compliquées et d'un aspect intéressant, faisant nouveau; ce travail, entrepris depuis bien longtemps, s'est toujours 
continué avec la patience nécessaire, et encore la connaissance approfondie des choses du métier, des dispositifs à employer, en un mot de tout ce qu'il faut prévoir à l'avance; c'est ce qui a produit d'innombrables armures dérivées, dont quantité ont été cataloguées dans les traités techniques de fabrication, telles que: Grains de poudre, Draps de Soie, Matelassés, et une infinité d'autres encore parmi lesquelles certaines qui n'ont fait souvent qu'apparaître et disparaître, affaire de mode, encore et toujours !

A ces armures fantaisie, on peut rattacher les artificès ingénieux de tissage, au moyen desquels on fait des tissus double face, ou sans envers; par exemple, une soierie satin des deux côtés, ou bien satin sur une face, et taffetas sur l'autre. Du même ordre encore est la fabrication de deux pièces directement superposées l'une à l'autre sur le métier et destinées à être séparées postérieurement; et aussi l'exécution de tissus tubulaires qui, terminés, s'ouvrent en tubes parfaits, de tissus circulaires; mais on n'en finirait pas d'énumérer ces cas particuliers où l'ingéniosité du fabricant et du tisseur se sont unies pour triompher des difficultés à résoudre; parmi toutes ces contextures diverses d'étoffes de soie, deux sortes, comme le velours et la gaze, réclament pour leur étude la place particulière que mérite leur importance. 


\section{LE VELOURS}

Le Velours n'a pas moins de titres de gloire que le satin, et c'est avec honneur et célébrité qu il a parcouru, depuis son invention, les contrées, les époques, sous des formes variées, rapidement belles et somptueuses, dont les vestiges sont les matériaux admirables que le temps a quelquefois épargné pour notre satisfaction.

Il est possible que $1 \mathrm{e}$ nom de cette étoffe vienne du latin : villosus, vcllus ou v'cllutum, par les formes archaïques : veluyau, vcluiau, velueau, velucl, veloux, velous, et dautres encore; mais, en tout cas, 1 idée première en fut certainement inspirée par la fourrure ou le pelage des animaux, épaisse et luisante couverture naturelle, qui dut éveiller de bonne heure la recherche imitative des premiers artisans tisseurs. Ceux de l'Inde et de la Perse furent encore, pour cette fabrication, des devanciers. L'Europe devait l'apprendre d'eux en s'initiant au luxe oriental, et c'est en Italie qu'elle allait prendre tout son essor et triompher, avec les velours de Venise, de Florence, de Lucques, de Gênes surtout, qui conservèrent longtemps la renommée d'être sans rivaux, particulierement aux temps fastueux de la Renaissance.

Grande avait été déjà la magnificence des velours au Moyen Age; certains, en France, étaient réservés à la puissance royale, comme le velours bleu semé de fleurs de lis d'or; d'autres étaient l'apanage de la chevalerie et des magistrats du rang le plus élevé. Au Grand Siècle, cette suprématie ne se démentit pas, chez nous, un instant: Marcelin Charlier, fondateur, en 1677 , de la Manufacture Royale de Saint-Maur-les-Fossés, fournisseur attitré du Roi Soleil, dont il pourroyait la magnificence en beaux velours et riches soieries de toutes sortes. mit le comble à sa réputation, en fabriquant pour Versailles un velours à parterre, sur un dessin de Bérain, enrichi de Soie frisée et d'or, qui revenait à I.ooo livres l'aune, et dont on ne pouvait fabriquer que dix-huit lignes par jour!

Au xviII siècle, les goùts de luxe qui affirmèrent l'usage du velours pour les vêtements des hommes dune certaine classe en augmentèrent la consommation, qui devint alors considérable et fut un encouragement à des recherches d'exécution et d'invention dans le décor et l'entente de cette étoffe. La beauté de ses reflets, sa lourdeur riche et coûteuse, devaient tout naturellement classer le velours en première place pour participer à des pompes officielles auxquelles il contribue encore aujourd'hui sous des dehors peut-être plus démocratiques, en même temps que la langue devait retenir cette réputation d'excellence par des locutions bien établies comme : faire patte de velours, jouer'sur le velours, marcher sur le velours, et cette autre plus familière: habit de velours, ventre de son.

Depuis un demi-siècle, le velours de soie, par une marche lente et sùre, une transformation graduelle de ses moyens de production, a été mis, on peut dire, à la portée de toutes les bourses. Il n'y a plus eu pour lutter 
contre une tendance trop forte, les sévères règlements corporatifs d'antan, prescrivant strictement le nombre, la grosseur, la préparation des fils de soie dans la fabrication des velours et jusqu'à leur largeur, leurs lisières et tous détails du même genre. Les traditions ont, pour ainsi dire, disparu, qui maintinrent, assez longtemps après que cette réglementation si minutieuse eut cessé, le tissage régulier à la main, confié à des ouvriers spécialistes du velours uni, en qualités dites : au fer - très souvent aussi désignées par les appellations de fabrique : 22,25 ou 30 portées; - ou bien elles se perdent de jour en jour en face des systèmes mécaniques de fabrication qui ont surgi et des besoins nouveaux qui se sont fait jour dans le commerce de cette étoffe. Cependant, il paraît à peu près indispensable de s'en rapporter à ce genre

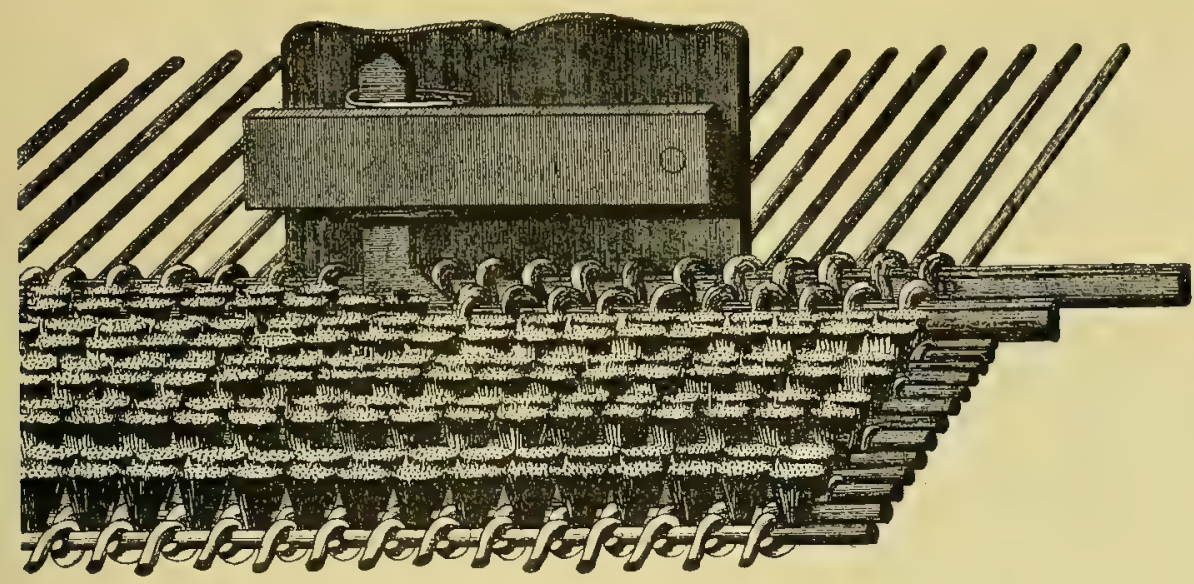

SCHÉMA DU VELOURS COUPÉ (d'après l'Encyclopédie).

ancien de tissage du velours pour donner de sa contexture une description de principe que la figure ci-jointe rendra sans doute plus claire.

Le velours comporte deux séries de fils de chaîne, ou plus simplement deux chaînes : celle dite de poil, destinée à former la partie veloutée proprement dite du tissu, et la chaîne de toile, qui, selon des exemples précédemment cités pour d'autres soieries, sert à composer le support de ce poil velouté, qui lui est rattaché intimement, lié par le travail du tissage. Une seule trame, qui peut n'être pas de soie (on fait quantité de velours tramé coton, etc.), suffit à lier les fils de chaîne de toile.

Cette fois, le tissu se fait endroit dessus, nécessairement; on en verra bientôt la raison. Le tissage du velours est un travail en quelque sorte alternatif : tantôt l'ouvrier ne s'occupe que du fond, en faisant passer la navette chargée de trame entre les fils de la toile; tantôt il fait lever tout entière la chaine de poil, jusque-là retenue entre les fils du fond, pour la faire passer par-dessus un fer, quil glisse adroitement sous la nappe de ces fils, dans le même sens que celui de la trame; ces fers, sortes de légères tiges métalliques, généralement de cuivre et d'une forme spéciale, comme on le voit sur la figure, se trouvent pris momentanément entre le fond lui-même et les fils de 
poil ; ceux-ci forment donc sur les fers autant de bouclettes très petites et serrées les unes contre les autres; alors, pour achever le velours, l'ouvrier procède à la coupe, lorsqu un certain nombre de fers, 2, 4 ou 6, ont été ainsi passés; elle s'exécute avec un rabot ou pince, instrument tout particulier, tenu à la main et dont la lame d'acier très aiguisée et coupante, introduite dans la rainure servant de guide que porte le fer à sa partie supérieure, et promenée habilement tout le long de cette rainure, tranche les bouclettes du poil en deux moitiés; retenues chacune à leur base dans le tissu de fond, elles forment à leur autre extrémité libre autant de fines houppettes soyeuses, courtes et pressées, dont la juxtaposition donne la partie veloutée.

Il est clair que plus les fils destinés à ce poil sont nombreux et d'une soie qui s'ouvre bien à la coupe, et plus naturellement le velours est beau, a vec une surface bien veloutée et fournie, que l'on égalise encore plus parfaitement en la soumettant à l'action d'une machine spéciale, dite à raser.

Il y a en somme une assez grande analogie entre le travail de confection des tapis de laine et celui du velours de soie, mais on peut se rendre compte, d'après les quelques détails ci-dessus, combien ce dernier est chose infiniment plus délicate et difficile, en nécessitant des précautions multiples et minutieuses. Cette fabrication n était confiée jadis qu'à des ouvriers expérimentés, très entraînés à ce genre de tissage et si habiles cependant à éviter la quantité de défauts qui peuvent trop facilement se produire, qu'ils arrivaient à une régularité de production étonnante, en mettant une sorte de point d'honneur à maintenir leur réputation. On a vu, à Lyon, autrefois, certains de ces ouvriers fabriquer une quantité totale de 1.500 mètres de velours de soie, ce qui comporte un travail d'environ dix années, pour une production moyenne, journalière, d'environ 60 centimètres, ce chiffre représentant un maximum, sans qu'il existât le plus petit défaut dans toute cette étoffe.

Les difficultés s'augmentaient encore avec les soins à prendre pour fabriquer des velours de coloris clairs au lieu de nuances foncées, moyennes, ou de noir; le comble de la délicatesse en cet ordre d'idées était représenté par la fabrication du velours blanc; un blanchiment parfait du fil de soie à la teinture est déjà une première difficulté dont nous avons parlé, et qui s'accentue ici,car il le faut, ce blanchiment, complet et intime pour ainsi dire, le fil coupé étant considéré par sa tranche et la teinte dans ce cas paraissant plus soutenue. Il fallait encore de plus éviter toute tache, tout contact trop prolongé des doigts au travail et tout ce qui pouvait ternir peu ou prou la soie, avec une dextérité toute spéciale dans la coupe, indispensable pour laisser au velours toute l'apparence de la meilleure fraîcheur de teinte, et comme je l'ai dit: toute sa fleur.

A ce propos, les annales lyonnaises ont recueilli une anecdote typique et asse $z$ curieuse.

Vers I 852, un sieur Thomasset, tisseur de velours, exerçait depuis trente ans sa profession avec une réputation d'habileté telle, qu'elle lui avait valu la qualification de roi des veloutiers.

Après avoir été pendant une longue suite d'années occupé à la confection de velours de la plus grande délicatesse, blanc, jonquille, rose clair, etc., il était arrivé cependant à un âge où l'on n'a plus la même dextérité et où il 
n'était plus possible de lui confier des tissus de pareilles teintes; on lui donna donc à fabriquer une pièce de velours noir. Thomasset ressentit une si vive impression de ce qui lui semblait être une dégradation, il éprouva un tel chagrin de déroger et de perdre le rang oủ l'avait élevé sa réputation de veloutier incomparable, qu'à peine eut-il mis sur le métier sa pièce de velours noir et tissé un demi-mètre, en proie à un accès de démence et de vertige, il se coupa la gorge avec un rasoir. Ainsi, selon les termes du narrateur de cette historiette, tout comme l'art culinaire, l'art du tissage a eu son Vatel!

Mais, nous le disions plus haut, le velours, à l'exemple de tant de genres textiles, a marché avec le progrès; il ne se fait plus, ou guère plus, avec la lenteur patiente du bon vieux temps. On a inventé des métiers fort ingénieux qui le tissent mécaniquement avec une régularité surprenante. Et ce procédé a eu l'avantage de diminuer beaucoup le prix de revient en augmentant considérablement la production; il n'y a pas eu simplement, en effet, l'accélération d'un organisme marchant par la vapeur, mais celle d'un mode de tissage par pièces superposées ou à doubles-pièces qui se fabriquent à la fois et nécessairement même.

Sans entrer dans les détails, qu'il nous suffise de dire que, pour deux toiles ou étoffes de fond, superposées au métier et se tissant à faible distance l'une de l'autre, il n'y a qu'un poil qui passe de l'une à l'autre des deux toiles, les relie par ce trajet lui-même jusqu'à ce qu'un rasoir mû automatiquement vienne trancher ce poil en laissant de la sorte une partie veloutée et finie à chacun des deux tissus du dessous et du dessus. L'économie de temps et de façon est évidente.

Et puis, au lieu d'employer toujours la soie pour composer ce poil du velours, on a obtenu, depuis longtemps déjà, des résultats très satisfaisants, en lui substituant la schappe, matière textile dont nous avons parlé à plusieurs reprises et sorte de soie secondaire; il se fait aujourd hui des quantités considérables de ces velours, poil schappe, à prix bas, et de grande vente pour tous usages: robes, manteaux, chapeaux, ameublements, etc. Il n'y a rien de comparable du reste dans la fabrication de ces velours inférieurs de prix et de qualité avec celle des velours coton, qui est basée sur de tout autres principes.

Il est assez curieux de noter qu'un essai dans ce genre de velours à bon marché avait été tenté dès le Xvirie siècle. Sous le nom de velours de Paris, une dame Pallouis, originaire de Lyon, fabriquait à Paris et vendait des velours faits en soie galette, genre de bourre de soie. Sa manufacture comptait au nombre des fournisseurs du Garde-Meuble Royal, en l'année 179.

Autre transformation moderne très remarquable du velours de Soie : à l'exemple de tant d'autres soieries on est arrivé à le fabriquer écru et à le teindre en pièces; et, après avoir redressé convenablement le poil coupé, assez éprouvé, on l'imagine aisément, dans ces opérations de teinture, et l'avoir remis bien en place, on obtient ainsi ces velours souples ou chiffons si goûtés actuellement pour les robes; c'est bien là un fait significatif par excellence, entre beaucoup d'autres, dans l'évolution textile.

Jusqu'ici nous n'avons envisagé que le velours poil coupé, mais il existe un autre genre de velours, dont l'usage en uni est à la vérité assez peu fré- 
quent, et qui est connu surtout par l'emploi que l'on en fait en accessoire et en combinaison dans les genres de velours façonnés à dessins : c'est le velours poil frisé, qu'on appelle aussi parfois épinglé, surtout quand il s'agit de rubans.

Dans cette sorte de velours, les fers employés au tissage sont différents de ceux décrits précédemment; ce sont de simples tiges métalliques rondes et polies, et, après les avoir passées, pendant le travail, sous les fils de poil, on les retire sans rien couper; les petites bouclettes de soie arrondies, qui ont été formées de la sorte, restent soulevées les unes à côté des autres et leur ensemble donne à la surface de ce velours l'aspect d'un gros grain

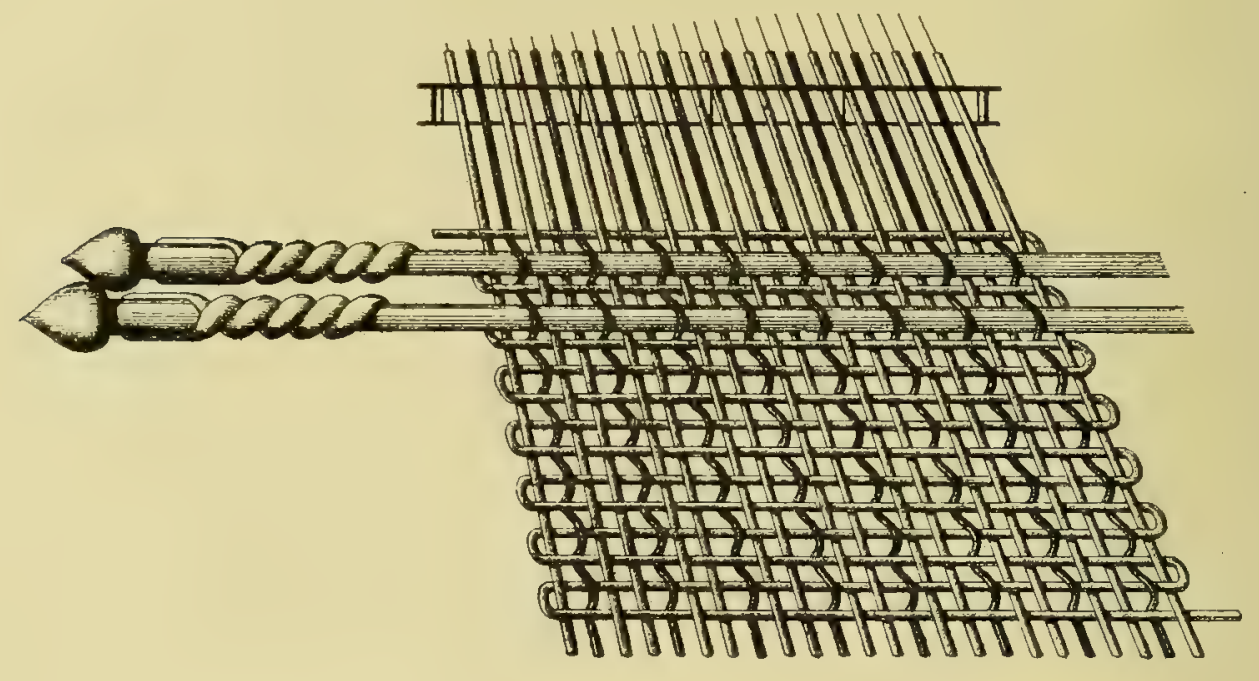

Schéma du Velours frisé (d'après Peyot).

particulier et d'une belle matité. Le contraste très heureux du velours frisé avec le velours coupé est utilisé depuis fort longtemps; cette opposition, comparable à celle du gros de Tours avec le satin, s'emploie à des fins décoratives similaires; du reste, le satin, le gros de Tours et d'autres armures peuvent être mélangés avec le velours pour concourir à la fabrication d'étoffes à bandes, à carreaux, ou à dessins. Il s'ensuit de tout cela que la catégorie velours est largement représentée parmi les soieries à décor et forme une classe importante sur laquelle nous aurons à revenir.

Nous avons dit plus haut un mot du parti de magnificence que l'on sut tirer du velours à des époques lointaines, en y mèlant des couleurs variées, en le rehaussant d'or, d'argent, en l'enrichissant de broderies, et souvent des plus magnifiques. Il est encore possible de gaufrer ou de frapper le velours, de l'illustrer par de l'impression, la peinture, etc.

Pour rester dans l'étude de ce tissu uni, disons que l'ingéniosité d'habiles spécialistes s'est attachée à faire des velours qui paraissent, au premier abord, irréalisables, par exemple : sans envers, présentant un velouté des deux côtés del'étoffe; ou bien, coupé sur une des faces et frisé sur l'autre; 
ce sont là, somme toute, des tours de force de tissage, demeurés dans le domaine de la difficulté vaincue, sans passer dans la pratique courante.

Quant au velours comportant un envers de satin, il a, bien au contraire, rencontré des applications nombreuses, si développées pour le ruban, par exemple, qu'il est devenu dans cette branche textile une spécialité, traitée de la façon la plus importante.

Cette rapide étude résumée serait par trop incomplète si nous passions sous silence des tissus veloutés qui sont les cousins germains du velours, soit le velours dit $d u$ Nord, et la Peluche. Est-il besoin de rappeler l'importance qu'a pu prendre, à notre époque, la fabrication des peluches, à voir de tous côtés l'emploi aussi considérable qui en est fait, aussi bien de celles de coton et de laine, que de celles de soie, pour de nombreux et variés usages.

On ne cherche pas, pour le velours du Nord, et encore moins pour la peluche, à obtenir un velouté très ras et fourni; au contraire, on s'arrange pour que le poil se trouve sensiblement plus long, et, pour cela, on forme la bouclette génératrice du velouté sur des fers plus hauts, et même de beaucoup, ou bien en augmentant l'écartement des deux pièces superposées au métier mécanique; ainsi, ce poil plus allongé s'incline une fois coupé sans pouvoir rester vertical, se couche même par places et produit les reflets chatoyants causés par la variété des jeux de la lumière, inégalement réfléchie sur cette surface mouvante et diversement teintée de ce fait.

En exagérant le couchage du poil d'une peluche dans un même sens, par le frottement et la chaleur simultanée, par exemple avec un fer à repasser ou une machine construite pour remplir le même office, on obtient la Panne, tissu aussi brillant que du satin, et plus brillant même; et c'est une qualité de panne, spécialement entendue au tissage pour être suffisamment mince et se lustrer particulièrement, qui forme la matière du cylindrique chapeau de soie; son prix et le degré de son élégance se mesurent aux reflets que le tissu soyeux dispense à ce couvre-chef, qui a ses détracteurs déterminés comme ses fidèles partisans. 


\section{LA GAZE}

Au velours, type de la soierie épaisse, fournie et lourde, s'oppose tout naturellement et inversement la Gaze; le nom est évocateur de légèretés et de transparences depuis qu'il fut attaché, si l'on s'en rapporte à la tradition, aux fins et légers tissus dont la fabrication fut florissante à Gaza, en Asie Mineure; leurs fils, d'après ce principe de la gaze, inspiré, au moins on peut le croire, par l'imitation des toiles tissées par les araignées et certaines chenilles, laissaient entre eux des.intervalles ménagés au tissage et suffisants pour rendre l'étoffe diaphane. Vraisemblablement, ces tissus furent les plus fins que l'on sut obtenir avec des fils de laine, de lin ou de coton, que l'on fabriqua dans l'île de Cos et dont on attribue l'invention à Pamphilia; l'époque romaine les connut sous les noms de ventum textile, nebulea linea ou togas vitrcas; du mème genre étaient ceux qui furent en usage en Egypte. Enfin Byzance n'ignora pas les gazes, non plus, paraît-il, que la Chine et encore l'Inde.

Un exemple intéressant de ces linons de l'antiquité nous est donné par les voiles exhumés des nécropoles, comme Antinoé; ils furent les ornements des Thaïs de ces époques reculées et, maintenant, sont objets de curiosité dans les musées; tant il est vrai que les légers tissus, les souples écharpes furent, presque de tout temps, les accessoires obligés de l'art de la danse.

Il faut supposer que, de fort bonne heure, on songea à utiliser la soie pour composer ces légers tissus et mieux quavec tout autre textile, en raison de sa finesse incomparable.

Quoi qu'il en puisse être exactement de ce point de départ des gazes, leur introduction en France paraît remonter au XIV $^{c}$ siècle et au moment où les papes résidaient à Avignon. Sans points de repère bien précis entre temps, on constate que ce genre de fabrication occupait, en 1667, à Paris une corporation d'artisans qui se dénommaient : Ferrandiniers Gaziers ou Gazetiers (la ferrandine étant une sorte de très léger taffetas). De I 770 à 1775 , on comptait, à Paris toujours, des milliers de métiers à fabriquer la gaze (on a dit qu'il y en eut trente mille). Ce nombre, vers 1783 , avait beaucoup diminué, la fabrication ayant reflué dans les provinces, notamment en Picardie et en Artois.

Peu à peu, des perfectionnements s'ètaient ajoutés en nombre les uns aux autres, dans la manière d'obtenir les gazes que l'on sut faire plus travaillées, plus ornées, enrichies d'or et d'argent, de perles, de cabochons, à l'imitation des pierres précieuses, dont ce fut un temps la coutume de les décorer.

Aujourd'hui, à ne considèrer que les seules gazes faites de soie, on trouve qu'elles présentent, en vue d'emplois très divers et très étendus, une très large variété qui est comme la gamme-type très développée du « chiffon » moderne.

On est arrivé, par le traitement surtout de la teinture et des apprêts, 
pratiqué après le tissage en écru, à donner à ces tissus leur souplesse inimitable et si recherchée; elle se prête admirablement aux élégances artistement chiffonnées, faites parfois d'un rien pour ainsi dire et qui achèvent agréablement une toilette, tandis que leur légèreté est fort seyante.

On a, du reste, l'habitude assez générale d'englober dans la même catégorie de gazes toutes les sortes de tissus, dès que leur finesse et leur légèreté les rendent plus ou moins transparents; cependant, à un point de vue plus étudié, il faut distinguer et séparer les gazes en deux grands groupes: gazes dites droites et gazes à fils de tour ou encore à tour anglais.

Dans les premières, l'effet de légèreté et de transparence est obtenu uniquement par l'espacement des fils entre eux dans le tissu; qu'on se propose de tisser un taffetas et que l'on constitue chaine et trame par des fils de soie assez fins, proportionnés d'ailleurs suivant le cas; que 1'on distribue les fils de chaîne, un à un le plus souvent, dans les dents assez écartées d'un peigne choisi ad hoc; que l'on réduise, c'est-à-dire que l'on serre en proportion les fils de trame et l'on réalise ainsi un tissu clair qui est de la gaze droite.

En réglant de façons différentes et dans des rapports variés ces écartements réciproques de fils, on dispose du moyen d'établir des qualités, des genres nombreux qu'on multiplie encore par le choix de la soie, sa préparation et par la manipulation adoptée, ou teint en fils, ou teint en pièces, ou même en gardant le tissu écru.

C'est ainsi que l'on a des gazes droites tissées avec de la soie grége pour rester telles; exemple: les anciennes gazes de Chambéry, qui eurent leur célébrité; les gazes pour cartouches, non moins démodées maintenant; les gazes à bluter les farines, toujours en úsage dans les moulins et minoteries, où l'on emploie également certaines gazes écrues aussi, mais à fil de tour.

Avec des soies moulinées, grenadinées par la torsion préalable, pour la plupart du temps teintes à l'avance, toujours tissées en taffetas, on obtient les souples grenadines, recherchées en noir pour des usages de deuils et bien souvent confondues avec d'autres gazes du genre canevas.

Mais, actuellement, le type le plus répandu de la gaze droite, c’est la Mousseline de soie. Elle est venue, presque récemment, sur les traces de sa devancière, la mousseline de coton qui se réclame, elle, d'une origine lointaine, d'un nom que l'on faic remonter à la ville asiatique de Mossoul, et qui fit la fortune de maints centres industriels, entre autres de Tarare, sous l'impulsion du trop ignoré Simonnet. Devant le succès rapidement triomphal de la mousseline de soie, devenue l'élue de la mode, atteignant en ces quinze dernières années à des chiffres fantastiques de production qu'il faut évaluer en kilometres, la concurrence n'était plus possible; le coton a dû céder le pas entièrement ou presque à la soie, d'une supériorité trop incontestable.

Formée de fils soyeux, croisés en taftetas et placés à légère distance les uns des autres, tant pour la chaîne que pour la trame, la mousseline se fabrique avec la régularité presque mathématique que procure le tissage du métier mécanique; on peut le vérifier, en regardant à la loupe, surtout une qualité tendue ou étirée par l'apprêt, et en voyant la rectitude des petits espaces carrés ou rectangulaires qui se trouvent ménagés entre les. fils. Remarque très importante, primordiale même : ces fils de soie écrue ou grège 
ont subi un moulinage tout spécial, une torsion généralement très forte qui varie dans une proportion indiquée par la pratique; il est commun de voir cette torsion atteindre jusqu'à 3.500 tours du fil sur lui-même pour I mètre de sa longueur.

Le résultat de toutes ces dispositions prises, c'est qu'à la teinture et sitôt la cuite opérée, la soie écrue perd son vernis naturel extérieur, son grès, comme nous le savons; elle devient souple, pas plus brillante que sa torsion forte ne le lui permet, mais en même temps, sous l'action de l'humidité et de la chaleur, les fils tendent à se détordre, par conséquent à se raccourcir, à la façon d'une ficelle que l'on a tordue entre les doigts et dont on ne maintient plus la tension lorsqu'on rapproche ceux-ci; et l'on obtient ainsi l'eftet particulier cherché de crêponné léger, de crêpage, trop connu pour qu'il soit utile de le décrire plus amplement.

Or, à ce moment, on est maître par l'apprêt de laisser à la mousseline à peu près tout ce crêpage et en même temps la perte de largeur et de longueur du tissu qu'il entraîne inévitablement, en un mot tout le retrait, ou bien de remettre l'étoffe tendue comme elle était en écru, avec tous les degrés intermédiaires de crêpage moyen, de souplesse, de toucher, de tenue, de tombant et cent autres caractéristiques d'apprêts différents; ces pratiques, chaque jour plus nombreuses, plus perfectionnées, combinées avec celles du tissage, grosseurs variables de la soie, nombre et sortes de fils employés, donnent une variété de mousselines si considérable qu'il serait impossible de s'engager dans le détail de leurs différences et de leur histoire.

Le hasard encore a joué son rôle dans le développement intense et récent de la mousseline de soie: en effet, par suite d'une erreur ou d'une négligence, un jour, une pièce, au lieu de recevoir l'apprêt très chargé de colle qui devait la rendre tendue et ferme, selon ce que la clientèle goûtait alors uniquement, fut apprêtée rien qu'avec de l'eau pure ou à peu près. Envoyée, toujours par erreur, à la vente avec une souplesse toute nouvelle, un aspect inconnu, considéré jusque-là comme un défaut à rejeter, cette pièce plut cependant par sa nouveauté, rencontra un emploi imprévu et finalement fut le point de départ d'une mode et du succès de l'apprêt chiffon et flou dont il est superflu de rappeler toute la fortune. Petite cause, grand effet, si l'on rapproche du fait très simple, initial, toute l'importance de la voie nouvelle ouverte dès lors à une branche industrielle et commerciale.

Telle que nous venons de l'esquisser, la mousseline est plus ou moins mate, en raison, encore une fois, de la très forte torsion communiquée au fil, ce point ne demandant pas de démonstration. Si l'on veut avoir de la mousseline qui soit brillante, il faut prévoir la chose dès le tissage et employer comme chaîne des fils en plus grand nombre, mais surtout sans aucune torsion ou grèges et toujours écrus, la trame pouvant rester tordue comme précédemment. Après la teinture, le tissu apparaît très brillant et un apprêt convenable lui donne la tension et la régularité qu'il faut pour l'emploi.

Par Voiles de soie, désignation très communément répétée maintenant, notons qu'on entend des tissus qui se réclament, soit de la mousseline comprise en qualités fortes, soit encore des grenadines teintes en pièces; d'autres voiles sont faits avec des fils de schappe également tordus; à tous on demande et par conséquent on donine le plus possible une suffisante 
épaisseur pour les rendre plus tombants et adaptés aux utilisations des robes, sans faire disparaitre toutefois la transparence qui est leur cachet.

Les usages de la mousseline de soie sont extrêmement nombreux; ce serait bien inutile de vouloir les énumérer. On s'est ingénié à mettre de cette mousseline unie un peu partout, de même qu'à la broder, la brocher, la gaufrer, la plisser, la peindre, l'imprimer, etc. C'est à ce point que l'on imagine malaisément aujourd'hui l'éclipse totale et surtout définitive de cette fort légère soierie, mais soierie quand même; toute impondérable qu'elle puisse être, elle a conquis en quelques années une si large place que cela fait oublier le rôle, bien effacé en comparaison, joué autrefois par les Organdis Jaconas, mousselines de 1'Inde, gazes Marabout, Marlis, Plumetis et d'autres encore.

Le défaut de certaines gazes droites est de ne pas offrir une contexture

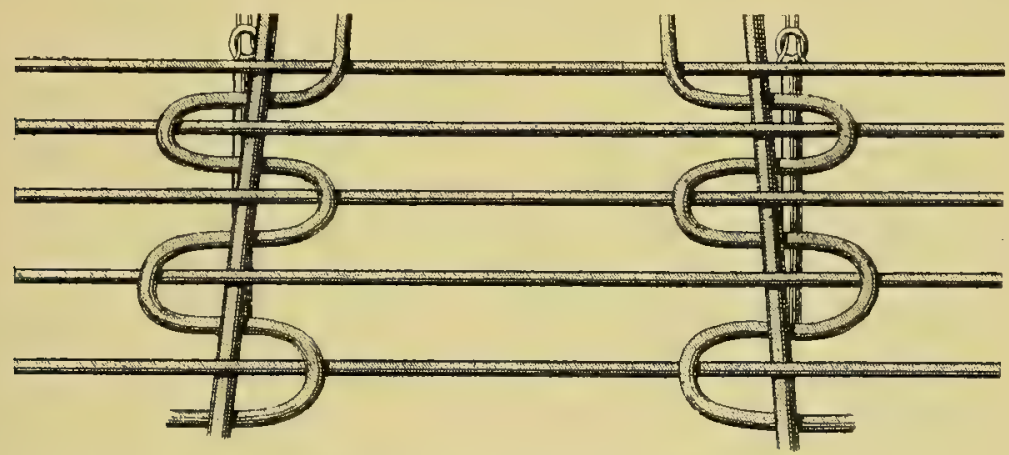

Schéma d'une gaze a tour anglais (d'après Peyot).

assez solide ni assez résistante aux déplacements des fils; écartés simplement les uns des autres, ceux-ci peuvent, sous un frottement trop brusque ou trop fort, glisser sur la trame en produisant la fàcheuse éraillure.

On a donc cherché à combattre ce défaut, et le système de la gaze à fils de tour ou à tour anglais permet d'obtenir une fixité meilleure des fils à leur place, tout en ménageant un ajourage bien marqué du tissu, en mème temps très régulier, ou quelquefois avec une irrégularité voulue. On fait alors s'enchevêtrer les fils d'une manière assez paradoxale à première vue, et le secours d'une figure est indispensable pour faire comprendre ce jeu mème dans le cas pris comme exemple et qui est le plus simple.

On distingue. sur ce tracé schématique des fils de chaîne dits fixes ou droits, qui ne sont appelés à subir aucun mouvement de levée; d'autres, au contraire, sont les fils dits de tour; intercalés entre les premiers, il arrive que, par un arrangement de lisses spéciales, ils lèvent tantôt à droite des fils fixes et tantôt à gauche des mêmes, en les contournant comme on le voit. A chaque fois, la trame insérée par la navette entre les deux genres de fils est prise dans la sorte de boucle formée; du fait qu'elle est retenue ainsi plus solidement, les jours formés par cette gaze ont plus de chance de rester intacts et de la grandeur qu'on leur a donnée. 
Cet ingénieux système du tour anglais, appliqué, retourné, combiné de cent manières, donne toutes les gazes qui, sous les noms de gazes $Z \dot{e} p h i r$, Diaphanes, Dona-Maria, à regard, Canevas, Etamines, etc., se fabriquent dans la région lyonnaise et dans le Nord de la France, en Picardie; la variété s'en complique par les mélanges comme trame de fils de laine, de coton, de schappe et aussi d'or et d'argent.

Dans la grande famille des gazes de cette espèce, qu'il est encore possible de décorer, comme nous le verrons, de dessins par le tissage ou par l'impression, relevons une sorte tout unie faite par le croisement simple ci-dessus indiqué par la figure; chaîne et trame sont d'importance à peu près équivalente, de soie écrue toutes deux; la teinture en pièces, puis l'apprêt, donnent le brillant discret et la souplesse à ce léger tissu qui a connu le succès avec une vogue soutenue depuis quelques années. 


\section{LES CRÊPES}

On accole si fréquemment les deux termes Gaze et Crêpe et on les prend si souvent l'un pour l'autre, sans doute parce qu'il existe un grand air de famille entre ces deux genres de tissus, qu'il est naturel de faire suivre leurs études.

Au reste, y a-t-il une démarcation vraiment tranchée entre les deux catégories? Certaines mousselines et certains crêpes arrivent à se confondre, d'après le système de leur confection qui repose, en premier lieu, sur l'emploi de soies spécialement et fortement tordues, en raison même des qualités que l'on envisage.

Il faut le dire et le répéter, le principe fécond de la torsion dite forcée donnée aux fils de soie est la base même de tout ou à peu près tout ce qui est crêpes ou crêpons, soit qu'on l'applique in tégralement à tous les fils employés, soit qu'on en use pour des mélanges et des combinaisons de fils tordus avec des fils qui ne le sont pas. D'oú résulte, au total, une variété presque inépuisable de tissus qui, pour la plupart encore, du fait en outre des manipulations dont on sait maintenant user à cette fin, présentent avec l'aspect crêpé, c'est-à-dire offrant les ondulations, les inégalités, les aspérités petites ou grandes que l'on a voulu déterminer et sur l'ensemble desquelles joue la lumière avec des clartés et des ombres de façon curieuse et très typique.

Cet ingénieux système de la torsion forte donnée aux fils de soie fut trouvé, sans doute, par les Orientaux et, en tous cas, par eux largement et habilement mis en pratique, comme nous allons le voir.

On s'accorde généralement à dire qu'il fut employé avec grand succès et une renommée bien établie à Bologne, ville où l'on faisait fort anciennement "beaucoup de soie retorse et autre 》 et aussi que, dès le XIV siècle, le crêpe était connu en France. Froissart, racontant l'entrée d'Isabeau de Bavière à Paris, parle d'un " deslié crespe de soie " couvrant la litière royale et " par quoy tout parmi on pouvait bien voir les joyaux », ce qui prouve encore la transparence de ce tissu. En I604, il s'établit dans la ville de Mantes une manufacture de crêpes fins, "façon de Bologne, crêpés et lisses de toutes sortes », qui eut quelque réputation; et bientôt cette fabrication se répandait en divers centres de tissage.

Les crêpes crêpés et les crêpes lisses se tissent toujours, peut-être par des moyens un peu différents de ceux d'autrefois; pour les premiers, la soie est bien plus tordue que pour les seconds, qui présentent moins l'effet de crèpés et sont surtout employés en ruches et garnitures de modes.

Le crêpe anglais, si connu par son application toute spéciale au deuil, est non seulement formé de fils de soie assez gros, espacés et très tordus sur eux-mêmes, mais, de plus, il reçoit un gaufrage particulier résultant de son passage contre des cylindres de cuivre gravés spécialement à cet effet; c'est là ce qui donne son grain caractéristique à ce crêpe, justement dénommé 
puisqu'il nous vint, en effet, d'Angleterre, oủ sa fabrication est toujours très active, avec une réputation bien établie.

Le genre de crêpe d'invention chinoise et si répandu maintenant sous le nom persistant de $c^{r} \hat{c} p e$ de Chine a donné lieu, en raison de l'ingéniosité de son établissement, et par le truc, si lion peut dire, de sa fabrication, à des recherches assez longues et patientes de la part de nos manufacturiers pour le réaliser tout pareil et aussi parfaitement.

Le crêpe de Chine est, en somme, la plupart du temps, un taffetas dont la chaîne est de soie écrue sans torsion ou grège, tramé alternativement de deux trames écrues également, à très forte torsion, et dont chacune a reçu cette torsion en sens différent, de droite à gauche pour l'une et de gauche à droite pour l'autre; on passe, au tissage, deux coups de l'une et deux coups de l'autre et toujours dans le méme ordre et ainsi de suite. Ce détail de fabrication, qui n’a l’air de rien, signifie, en réalité, beaucoup.

Au décreusage ou cuite, en effet, première opération de la teinture du tissu, la soie perd son grès; les fils, ceux de la chaine surtout, devien. nent souples et brillants; ceux de la trame, eux, se détordent, se retirent sur eux-mèmes, comme ciest le cas pour la mousseline et de façon plus accentuée encore. Et, sil n’y avait eu qu'une torsion d'un seul sens employé, l'effet de crèpage se produirait en déterminant, d'après un fait d'expérience quion utilise d'ailleurs, la formation de côtes longitudinales ou sortes de plis saillants, ce qui n'est pas ce que l'on veut obtenir dans le cas présent: au contraire, les torsions inverses, employées comme dit cidessus, s’équilibrent dans leur détordage et leur retrait si intimement, que le résultat produit est, au total, un crêpage fin, régulier et, en quelque sorte, discret. L'apprèt donné avec soin vient, de plus, finir le tissu, lui donner la largeur voulue, comme le toucher et l'épaisseur convenables.

Il est presque inutile de dire quaujourd hui les crêpes de Chine européens ont une très grande supériorilé de finesse, de régularité, de brillant sur leurs rivaux vraiment chinois restés lourds, grossiers, irréguliers et moins propres à recevoir la teinture en toutes nuances.

Aussi, les crèpes de Chine comptent-ils au rang des soieries favorisées de la Mode, quand celle-ci recherche de préférence, pour le costume, les étoffes souples et vaporeuses et comme finement soyeuses, toutes qualités qu'ils détiennent au plus haut degré, et que l'on a variées, multipliées par différents modes de tissage et de croisement des fils, autant que par la diversité des manipulations consécutives de teinture et d'apprêt.

Crèpes et Crêpons sont cousins germains, ces derniers ayant toujours pour principe le retrait produit par les matières textiles très tordues sur elles-mêmes, en combinaison avec d'autres moins tordues ou sans torsion; il faut dire matières textiles, car à la soie se mélangent fort bien, dans le cas, la schappe, la laine, le coton. L'imagination créatrice des chercheurs s'est largement donné carrière dans cette voie, ce qui explique la quantité de tissus de ce type crêponné qui offre, à des emplois nombreux, des ressources très appréciées.

La trouvaille remarquable du chimiste anglais Mercer, observant le premier que des fils de coton convenablement choisis, plongés dans une solution alcaline de soude caustique, y éprouvaient une perte de longueur ou 
un retrait pouvant atteindre I 2 à 20 pour IoO, fournit de nouvelles armes aux producteurs de crêpons. En unissant, par exemple, dans un même tissu et par parties ou rayures juxtaposées de la chaîne, la soie d'une part et de l'autre le coton, on obtient après mercerisagel un crêpon à bandes: celles formées par les fils de coton, qui ont subi seuls le rétrécissement causé par l'opération, sont naturellement bien tendues et, à côté, celles faites de soie qui, ne pouvant garder toute leur étendue, entrainées qu'elles sont par le raccourcissement du coton, paraissent ondulées et comme gaufrées.

On peut donner à la mousseline de soie l'aspect crêponné par un crêpage spécial à ce tissu; on le lui fait subir lorsqu'il est écru en le faisant passer un peu de biais entre des cylindres de bois garnis de peau; sous cette action, la mousseline serrée et presque régulièrement froissée prend une apparence brouillée et crêponnée qui se confirme à la teinture et à l'apprêt.

Bien entendu, selon la formule plusieurs fois répétée précédemment, crêpes, crêpes de Chine, crêpons divers, peuvent parfaitement se broder, s’imprimer, se façonner de nombreuses manières, ce qui achève de rendre très intéressante et importante cette classe de soieries légères.

1 Lorsque l'on applique le mercerisage aux fils de coton en flottes, avec le soin d'empécher par des dispositifs appropriés le retrait habituel de se produire, il arrive que ces fils gagnent á cette opération un brillant et une solidité très augmentés ; c'est tout le principe du similisage, système qui se développe chaque jour et produit le coton similisé ou similisoie qui a trouvé de trés larges applications textiles. 


\section{TULLES DE SOIE}

Il n'est pas possible de passer sous silence, au chapitre des étoffes de soie ajourées et très légères, les Tulles, réseaux, qui se présentent actuellement sous les aspects les plus divers, depuis limpalpable tulle illusion ou tulle maline jusquaux fortes et lourdes mailles, aux grosses guipures et aux dentelles de soie mécaniques.

A lorigine, les tulles furent exclusivement faits avec du fil de coton. Cette fabrication, tentée à l'imitation des travaux de la dentelle à l'aiguille ou au fuseau, prit naissance en Angleterre, à Nottingham. On rapporte que vers lannée 1600 la première machine aurait été inventée dans cette ville et construite par le révérend Lee, curé de Calverton, qui s'était fait mécanicien. Lee vint en France présenter son invention à Henri IV qui le protégea; mais à la mort du roi, Lee fut oublié et mourut obscurément; les ouvriers anglais qu'il avait amenés avec lui reprirent le chemin de l'Angleterre et dotèrent leur pays de lindustrie que nous dédaignions.

Une autre version, plus généralement acceptée, fait dater de i 768 l'invention du premier tulle fabriqué à Nottingham sur le métier à tricoter les bas par Hammond; ce tulle n'était à la vérité qu'une sorte de réseau imparfait, ressemblant assez à ce que l'on appela depuis fond de Bruxelles; ce tricot se défaisait à la rupture d'une seule maille.

C'est à Heathcoat qu'il faut attribuer la création de la véritable machine a tulle, et longtemps cette fabrication sembla devoir rester le monopole de l'Angleterre. Pour défendre ce monopole contre les entreprises de l'étranger, il fut absolument interdit, cela sous des peines extrêmement sévères, a-t-on dit, d'apporter d'Angleterre sur le continent le métier à faire le tulle. Cependant, l’intérêt, l'appât du gain, devaient être plus forts que toutes les défenses. Colbert avait bien réussi, malgré les terribles menaces et les tragiques représailles de la République de Venise, à lui arracher le secret de la fabrication des glaces.

Pièces à pièces, au prix de mille dangers de poursuites et de surprises, un premier métier fut, vers 1816, apporté et monté en France; le vrai premier tulle mécanique ainsi fabriqué reçut une broderie à la main et on en fit une robe, offerte à la Duchesse d'Angoulême. L'établissement d'autres métiers suivit bientôt à Calais et, dans cette ville, l'industrie du tulle progressa d'abord lentement. L'Angleterre gardait encore la supériorité des fils de coton quion y filait mieux et plus finement que partout ailleurs. Mais bientôt, vers 1823 , le premier métier fabriqué en France put fonctionner. La mode du tulle fit fureur de I $82+$ à 1826 et, sous cette influence, la fabrique calaisienne prit un développement considérable.

D'ailleurs, la fabrication du tulle, en se perfectionnant toujours, s'était implantée dans d'autres centres, à Caudry par exemple; elle se développait à Nîmes, à Lyon, où l'on avait appliqué le système à fabriquer le tulle fait 


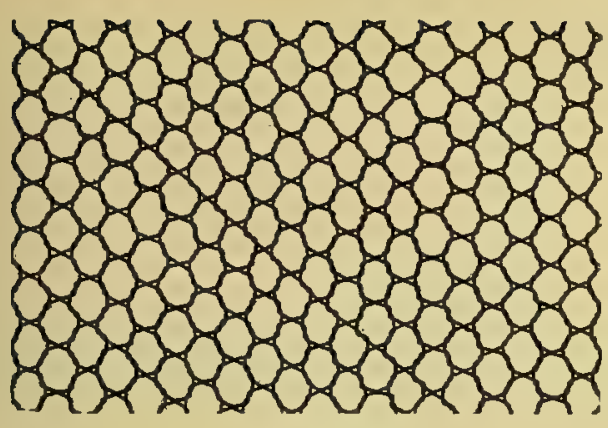

TULLE ALENÇON.

(Grossi.)

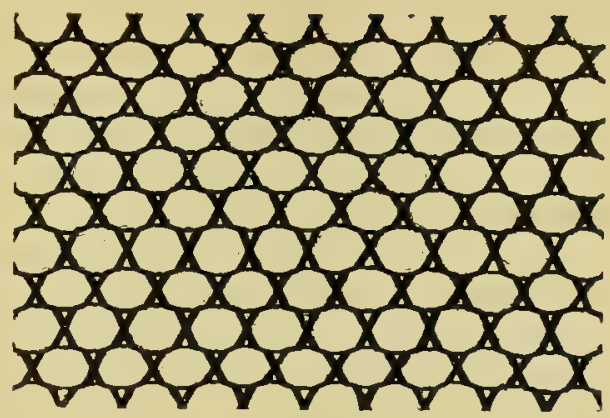

TULLE GREC.

(Grossi.)

avec de la soie surle métier à bas à partir de I791. Bientôt on devait parvenir au fonctionnement mécanique des métiers d'Heathcoat primitivement mus à bras d'hommes.

Aujourd'hui, les réseaux de tulles les plus légers, les plus aériens, ceux que l'on fait pour voilettes, avec le moins possible de fils de soie très ténus, sont tissés sur des métiers mécaniques de très grandes proportions et fournissant du tulle en largeur de plusieurs mètres. Le fer et l'acier les composent exclusivement et en font des "organismes pesants, compliqués et en même temps délicats, dont la figure ci-contre pourra donner quelque idée. Leur prix atteint facilement 30.000 et 40.000 francs. On comprend que, pour amortir ce coût élevé et couvrir tous les frais d'exploitation, il serait nécessaire de faire sortir de ces métiers des quantités incroyables de tulle à dix sous le mètre par un travail incessant de jour et de nuit.

Nous n'entreprendrons pas de décrire par le menu les divers tulles unis, Maline, Alençons, Chantillys, Filets, etc., ni comment les fils s'y croisent entre eux, d'après divers procédés, pour former les mailles rondes ou polygonales que 1'on con proportions différentes mailles, comme de gros forment. Le mieux, pour de la fabrication du tionner la machine sous principe ces mailles s'ob ments d'un grand nom ou bobines circulaires et qui sont garnies de fil et circulent entre d'au verticalement; le fil des en en faisant le tour, ganes du métier vien la mettre en place et Tous ces mouvements, avec une précision d'a dixième de millimètre,

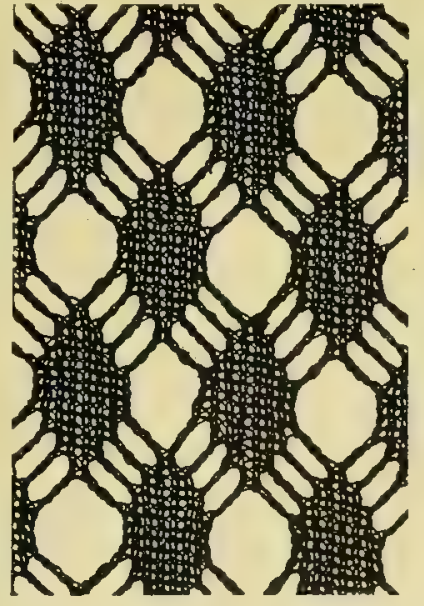

TUlle dit a la chaine. (Grossi.) naît, avec toutes les de grandeur pour ces seur pour les fils qui les se faire une idée juste tulle, est de voir foncses yeux; disons qu'en tiennent par les mouvebre de sortes de navettes aplaties, dites chariots, de soie à leur intérieur tres fils de chaîne tendus navettes se lie à ceux-ci tandis que d'autres ornent terminer la maille, calibrer sa grandeur. automatiquement réglés justage atteignant le se succèdent cependant 
avec une rapidité étonnante quand on songe à la disproportion de tant de fer et de si peu de soie.

Tout naturellement, la teinture et lapprêt jouent le rôle le plus important dans la terminaison des tulles dont la majorité est tissée avec de la soie écrue. C'est l'apprêt régulateur qui replace correctement les fils, les tend pour donner à la maille sa forme correcte, pour la cadrer, selon l'expression

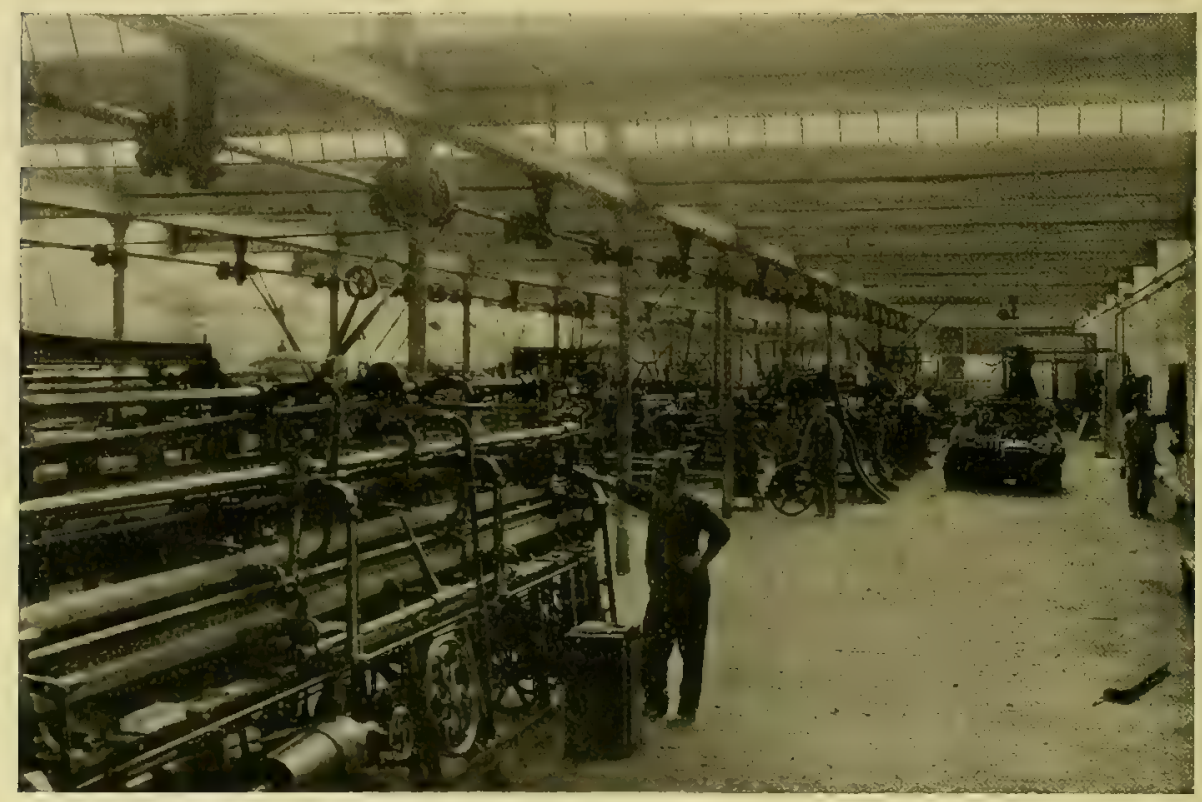

Métiers mécaniques de Tulle !.

consacrée, et donner aux tulles divers l'aspect et la tenue que réclame leur vente.

Ensuite, c'est encore nombre de transformations et d'enjolivements qu'on ajoute fort souvent aux tulles unis. Pour rendre la voilette plus attrayante, on l'orne de mouches ou de pois de chenille ${ }^{2}$, placés à la main. D'autres genres sont ornés d'applications, gouttes de gomme pure ou colorée, que l'on dépose ingénieusement sur le tulle et qui forment, une fois séchées, des perles brillantes imitant le cristal, le métal, le jais, etc.

Bien connus sont les tulles pailletés, rehaussés de cet ornement : la paillette, empruntée à la broderie qui sut en user fort longtemps.

1 MM. Kiemlé et Marcet, à Lyon.

2 La chenille qui sert à moucheter ou cheniller les tulles est une sorte de cordon velouté plus ou moins gros, que l'on tisse avec une machine ad hoc et dont l'âme intérieure est ordinairement faite d'un fil de laiton fin ; cela permet de la couper à la grandeur voulue et de l'adapter aux endroits choisis, en tordant avec une dextérité acquise par l'habitude le fil métallique autour des fils de soie. 
Il était logique, quaprès avoir résolu le problème de fabriquer le tulle uni, on s'attaquât à celui évidemment plus compliqué de faire ce réseau avec des dessins tissés au métier. Après maints tâtonnements, l'invention de Jacquard apporta le moyen pratique de solutionner la question. Ferguson, en I836, parvint à adapter la fameuse mécanique au métier circulaire et produisit le tulle façonné, dit Dentelle de Cambrai ou imitation de Chantilly; toute l'industrie de la dentelle mécanique est venue de cette ingénieuse application.

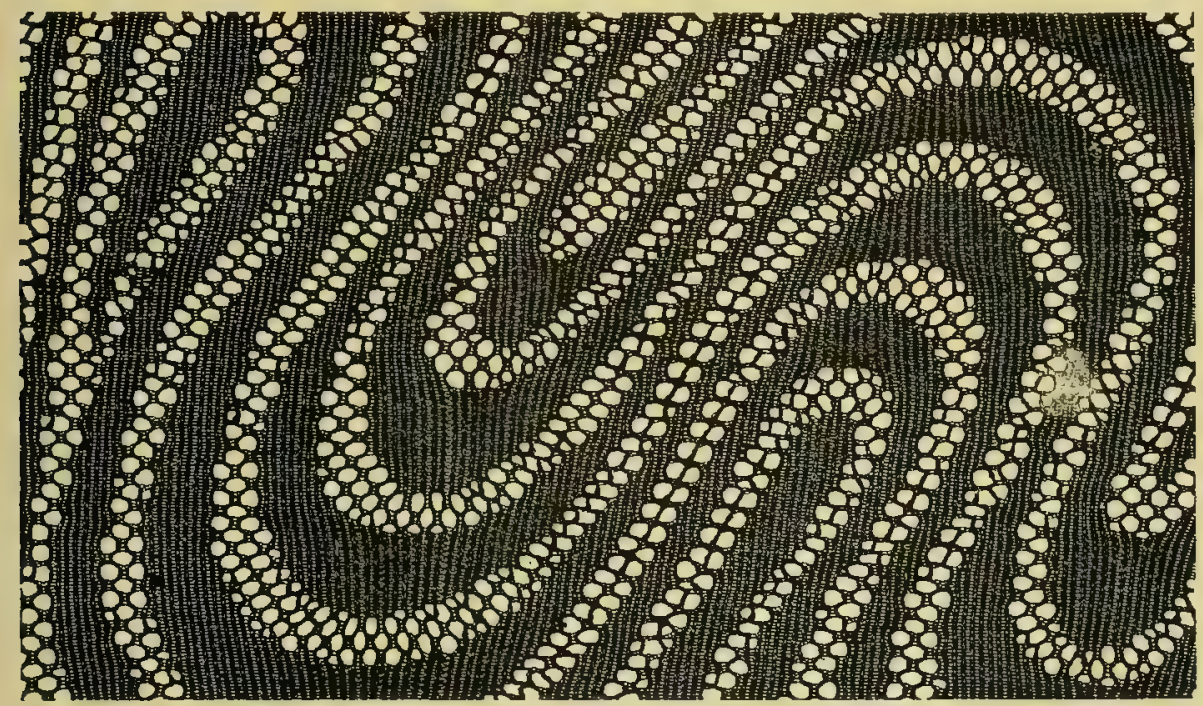

Tulle façonní. DIT A la Chaine (fabrication de Lyon ${ }^{1}$ ).

Il existe maintenant plusieurs ingénieux systèmes de métiers permettant d'obtenir des tulles façonnés et de produire à volonté à des places déterminées des mailles ajourées de différentes sortes et grosseurs mélangées, en contraste avec d'autres parties où les fils plus serrés, plus redoublés, parfois en relief à la façon du broché, donnent un tissu plus fourni, plus épais, en imitation de la dentelle ou de la broderie; l'un des plus répandus est celui qui fonctionne d'après le système Leavers. Parmi tout ce qui se fait à Calais et à Lyon comme tulles façonnés, est-il bien nécessaire de donner des exemples? L'écharpe ou la mantille, accompagnement jadis obligé de la toilette d'une Espagnole, en est un très connu, tout aussi bien que ces imitations de Chantilly et ces voilettes à dessins dont la variété est aujourd'hui si grande.

On a tiré aussi un parti excellent du métier fort ancien de principe, métier à faire des bas ou des gants, dit à la chaîne ou 4 rangs; dans ce métier, les fils de soie se nouant entre eux d'après l'antique procédé du tricot

1 Communiqué par MM. Vial et Ci^, à Lyon. 
arrivent à manœuvrer si bien quion peut exécuter de la sorte des dessins interprétés par la diversité des mailles produites et des pleins ou mats s'enlevant sur les rides des réseaux à jour. Et ces genres complètent une classe de tissu soyeux d'une très grande variété que la mode favorise par intervalles de ses préférences et pour la garniture des robes et pour celle des chapeaux.

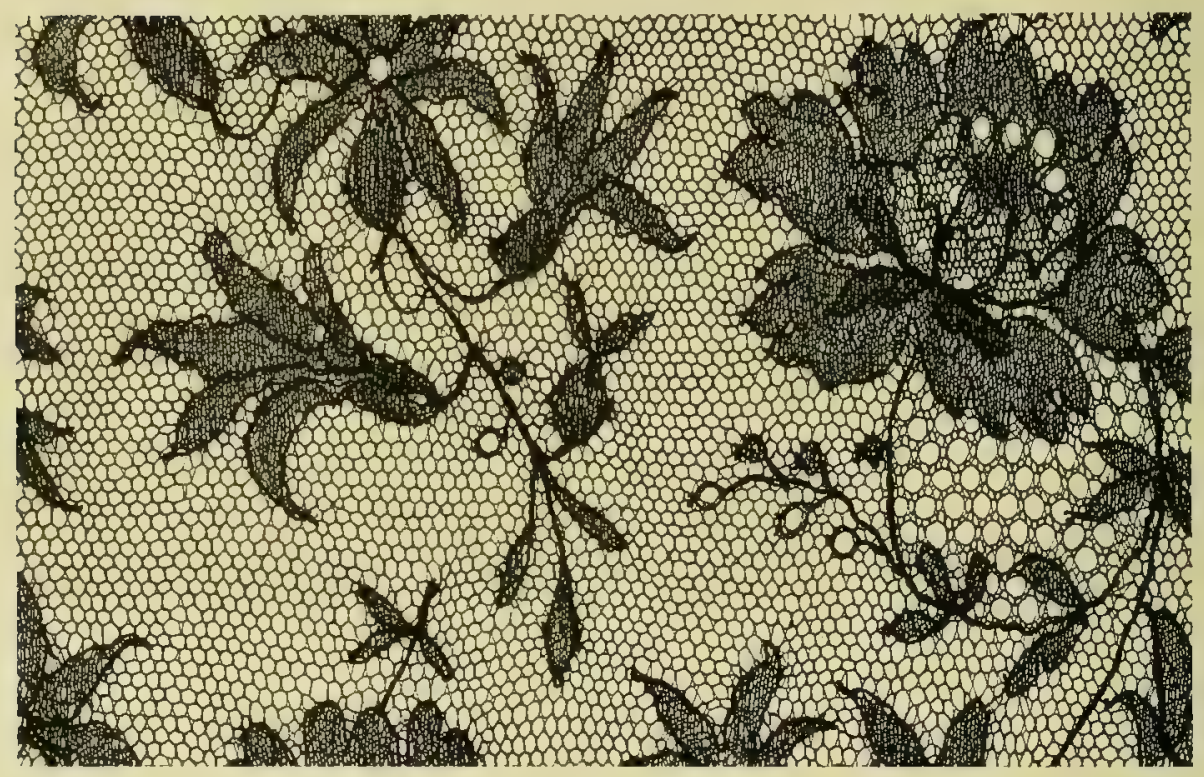

Tulle façonné (imitation de Chantilly), fabriqué avec le métier Leavers. 


\section{LES SOIERIES A DÉCOR}

Après avoir passé en revue les principaux types de soieries unies, celles qui tirent leurs caractères et caractères, limités par conséquent, des croisements de fils représentés par les armures diverses produites sur le métier d'uni, il est temps d'en venir à l'étude des soieries à décor et des moyens qui permettent de les obtenir. On a vu qu'au cours des lignes précédentes nous avons anticipé quelque peu, à plusieurs reprises, et forcément, sur cet important chapitre; parler, en effet, par exemple du moirage, du gaufrage, c'est envisager des procédés qui donnent bien, aux étoffes auxquelles on les applique, un réel décor, en modifiant, transformant leur aspect primitif ; l'impression, la peinture sont du mème ordre et mériteront, du reste, un développement particulier, aussi bien que la broderie qui a un certain rapport direct avec l'art même du tissage et possède de glorieuses références.

Cela revient à dire qu'il convient d'envisager la décoration de l'étoffe de soie dans un sens large, pour les moyens employés comme pour les résultats obtenus. Former, par exemple, dans un taffetas des rayures de couleurs différentes du fond, faire alterner dans un mème tissu et par bandes le gros de Tours avec le satin, le satin avec le velours, etc., c'est déjá faire de la décoration; et ce fut bien là, sans doute, le procédé le plus anciennement imaginé et employé pour illustrer une étoffe et en augmenter l'intérêt, comme le point de départ d'un ensemble devenu, avec le temps, d'une importance aussi considérable et d'une extrême complexité.

Et les soieries à rayures, bandes, filets, carreaux étant ainsi le prologue obligé de celles qui sont façonnées mieux encore et avec plus de diversité et plus de complication par le tissage, au point que ce travail arrive à former des dessins, il est tout naturel d'apporter quelques précisions sur ces premiers genres, que l'on peut réunir, si l'on veut, sous l'appellation générale, et d'une assez commode imprécision, de soieries " fantaisie ». 


\section{RAYURES ET CARREAUX}

Il est relativement simple de former sur une étoffe de soie des bandes ou rayures, régulièrement ou irrégulièrement disposées, quand elles ne se distinguent du fond de cette étoffe que par leur nuance différente et que le croisement des fils reste le même dans la largeur totale; cela s'obtient par le choix des fils de la chaine pris en nombre voulu et calculé pour chaque couleur à l'ourdissage.

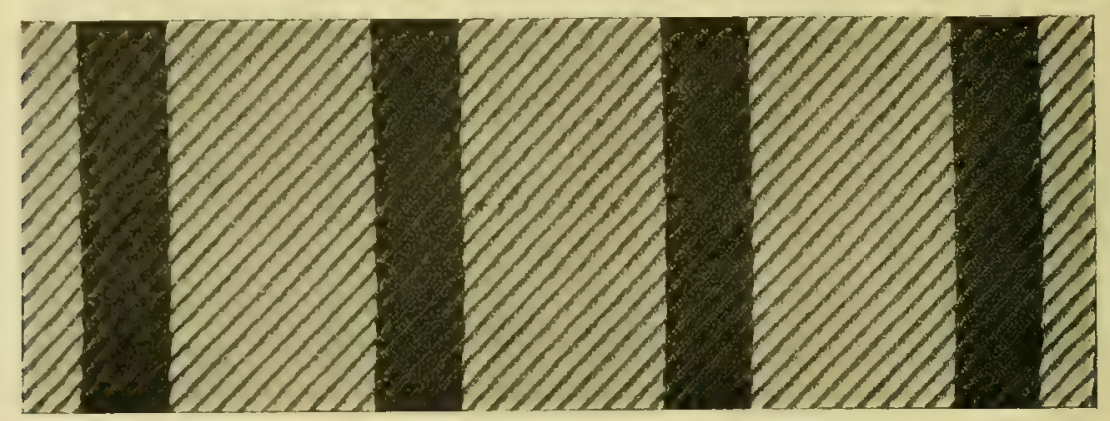

SOIERIE RAYÉE PAR BANDES DE TEINTES DIFFÉRENTES.

11 est bon cependant et presque indispensable d'avoir tracé à l'avance sur le papier la disposition des rayures que l'on se propose d'obtenir, en l'étudiant au point de vue de la largeur de ces rayures et de leur arrangement régulier ou irrégulier d’après l’étoffe que l'on veut faire et son emploi : robe, ameublement, doublure, etc.

L'habitude vient en aide pour réaliser un placement heureux de ces rayures et le meilleur effet à produire, en leur donnant aussi un coloris agréable à l'oeil : nuances de tons opposés et cependant allant ensemble, tons camaïeux ou dégradés de la même teinte ou encore ombrés. Mais, en même temps, il est indispensable de ne pas négliger la règle technique du rapport, notion qui s'impose déjà en l'espèce et que nous allons voir figurer comme un point de début de toute importance, en parlant plus loin des dessins et soieries façonnées.

En matière de rayures, est-il besoin de faire remarquer ce qui est l'évidence même? A part de rares exceptions, par exemple lorsqu'il s'agit de rayures employées en bordures, en encadrements, vers l'une des lisières du tissu ou vers chacune d'elles, celui-ci, considéré dans une largeur moyenne et courante de 50 à 60 centimètres, comporte forcément ou presque toujours la répétition des rayures, dont chacune présente des dimensions moyennes et mème plutôt petites, que les rayures soient régulièrement ou irrégulièrement 
placées sur le fond, point à noter en plus. Or, pour que cette répétition ait lieu correctement, qu'elle se produise à des places prévues, si bien que le résultat d'ensemble soit juste pour la largeur entière de l'ètoffe et que le raccordement des lés à la couture soit convenablement assuré à chaque lisière, il est indispensable, cela tombe sous le sens, qu'on ait calculé tout au préalable. Et l'on peut définir le rapport de disposition de la rayure envisagée, en le considérant comme le rapport de la largeur minima évaluée en centimètres, si l'on veut, qui est nécessaire et suffisante pour tracer et exécuter une première fois la rayure avec les nombres de fils voulus, $\grave{a}$ la largeur totale de l'étoffe qui est complétée en répétant ou en faisant courir la disposition.

Il n'est pas possible de laisser de côtó ces considérations, qui paraissent

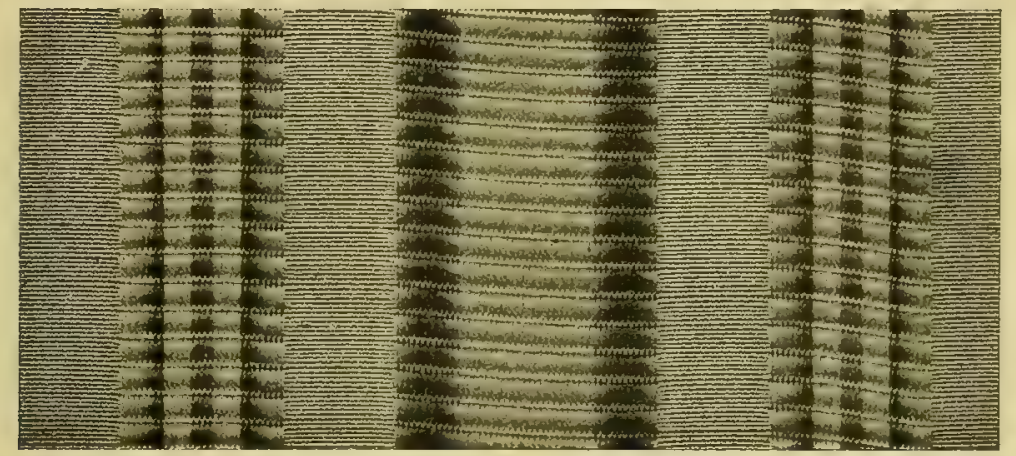

SOIERIE RAYÉE PAR baNdes D'ARMURES ET DE TEINTES DIFFÉRENTES.

des minuties arides et strictes, dont on ne se préoccupe guère quand on regarde tout simplement une étoffe à bandes, car si l'on négligeait ces soins nécessaires, on risquerait inévitablement de produire des étoffes présentant un défaut de construction très apparent; elles seraient, en quelque sorte, boiteuses, par suite inemployables.

Les soieries à rayures sont nécessairement plus compliquées lorsque ces rayures sont faites par des armures différentes et que l'on combine par bandes le taffetas ou le reps avec le satin, le satin ou le gros de Tours avec le velours, la mousseline, la gaze, et ainsi de suite. Il importe alors de tenir compte des grosseurs de fils de chaîne convenables pour chaque armure, de leur répartition sur le métier et des précautions à prendre pour l'exécution simultanée de croisements différents par parties voisines et rattachées les unes aux autres. Ainsi, les fils qui forment du satin et ceux à côté du taffetas n'ayant pas le même croisement les uns et les autres éprouvent, par suite, un retrait de tissage ou embuvage qui n'est pas le mème pour les deux groupes de fils; d’où nécessité de séparer ces deux groupes destinés soit au satin, soit au taffetas et de leur laisser une indépendance relative en les disposant dans la pratique sur deux rouleaux au lieu d'un seul à l'arrière du métier, faute de quoi, et très vite pendant le travail, les tensions deviendraient inégales et désaccordées; de ces fils, les uns tireraient et casseraient, 
les autres deviendraient trop lâches; la fabrication du tissu serait fort défectueuse et deviendrait même impossible.

Et, plus sont différentes les armures que l'on oppose en contraste heureux, justement à cause de leur diversité, plus il est nécessaire d'apporter à leur combinaison parallèle des soins spéciaux et un sens averti de l'organisation du tissage et de la façon de comprendre le tissu, suivant qu'il est tissé teint ou teint en pièces.

On désigne très généralement maintenant les soieries à rayures sous le nom de Pékins ou Pékinés, cette désignation venant probablement des étoffes de ce genre fabriquées en Chine, mais on ne saurait l'affirmer avec certitude, car des auteurs font ressortir qu'on appelait pékins, au XvIII siècle, des sortes particu peints ou à fleurs. englobent tous les de combinaisons duire des rayés leurs et d'armures, et encore par une tières textiles, soie coton, ou fil, soie et Le plus souvent. produites sur le ment fines, on nom de pékiné, Millc

A u lieu de le sens de sa lon moyen de la chaí

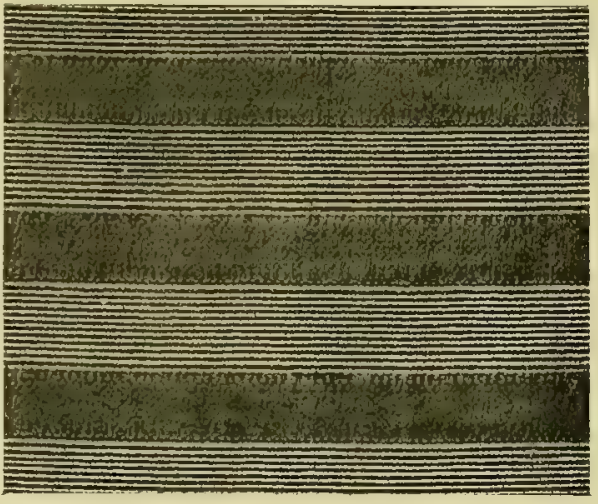

SOIERIE BARRÉE, OU BAYADÉRE, PAR DES ARMURES DIFFÉRENTES. lières de tissus Les pékins actuels nombreux genres en usage pour propar effets de coucomme ci-dessus, opposition de maet laine, soie et or ou argent, etc. quand les raies tissu sont notableme le genre, au lieu raies ou Fileté. rayer le tissu dans gueur et par le ne, il est encore possible de faire que les rayures se produisent dans le sens transversal de la largeur. On obtient cet effet aussi bien par des trames de nuances variées qui se succèdent dans un ordre donné que par des changements d'armures à intervalles réglés; pour prendre un exemple de ce dernier cas, il est très possible de faire alterner l'armure du satin s'exécutant dans toute la largeur de l'étoffe avec l'armure gros de Tours. L'effet de ces diverses combinaisons est dit alors barré et on appelle souvent aussi Bayadères tous les genres de soieries ainsi faites; le décor placé de la sorte en travers exige, bien entendu, pour une adaptation agréable au costume que sa forme se prète à cette disposition qui fut goûtée particulièrement avec les crinolines et les robes à volants du Second Empire.

Le corollaire de la rayure est naturellement le carreau. Que l'on prenne, en effet, par exemple, un taffetas pékiné ou rayé régulièrement noir et blanc, il suffira de le tramer alternativement avec du noir et du blanc et, chaque fois, avec le nombre de coups de trame nécessaire pour que cette rayure en travers forme avec celle en long un taffetas carreauté régulier ou damier. Il est à remarquer que, des carreaux formés, les uns seront nettement noirs, fils de chaîne noire avec trame noire; d'autres, nettement blancs, chaîne blanche, trame blanche; et d'autres, intermédiaires, mélangés forcément de fils de chaîne noire et de la trame blanche ou inversement, donneront à ces places un ton de grisaille ou de glacé qui ne nuit pas à l'harmonie de l'ensemble. 
Le carreauté ou quadrillage régulier est la base de tous ceux que l'on obtient en assortissant des soies de couleurs différentes, comme aussi en combinant des armures qui les fassent jouer pour leur meilleur aspect. C'est d'après ces données que l'on a fabriqué les soieries écossaises, dans lesquelles des teintes, le plus souvent vives, même très vives, sont mêlées à l'imitation des célèbres et très nombreux tartans des clans d'Ecosse. Ce genre a bénéficié maintes fois d'une vogue très marquée; on sait combien l'imagination s'est donné carrière pour créer une variété innombrable de carreaux, depuis les plus petits et les plus imperceptibles jusqu'aux plus grands et les plus osés, simples ou compliqués, réguliers ou irréguliers, agrémentés parfois de filets ou bandes de satin, de cannelé, dans le but de relever encore le caractère voyant de ces soieries.

On remarquera, au total, que les tissus de soie à rayures ou à carreaux peuvent atteindre à une complexité comme à une recherche non négligeables, alors que l'on serait tenté, au premier abord, de les tenir pour des sortes très courantes et de ressources limitées. D'ailleurs, on reste étonné, émerveillé même, en voyant la quantité considérable de choses charmantes et du meilleur goût que les fabricants lyonnais du XVIII ${ }^{\circ}$ siècle surent exécuter en tirant parti de l'idée de rayure, tournée, retournée de mille façons, avec autant de justesse dans les proportions que d'habileté technique, aussi bien dans les plus simples "petites soies » à l'usage de doublures fabriqués en ce temps-là que pour les étoffes moyennes et riches destinées soit au costume soit à l'ameublement.

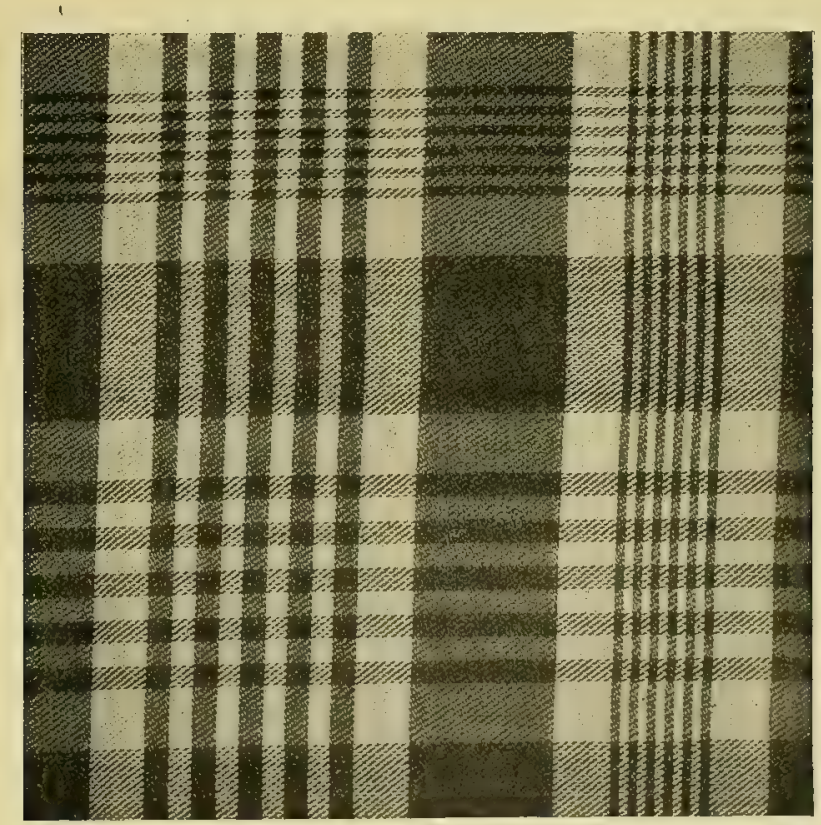

SOIERIE QUADRILLÉE.

(Communiqué par MM. Claude frères, Paris.) 


\section{LES SOIERIES FAÇONNÉES OU A DESSINS TISSÉS}

Façonner un tissu veut dire, de longue date, le décorer, l'orner de dessins par le tissage; à telles enseignes que l'on désignait autrefois sous le nom de faconnier en soie l'ouvrier qui fabriquait ce genre de soieries, comme on appelait marchand faconnier en soie celui qui préparait les soies pour être employées à ces étoffes faisant l'objet de son négoce. Et, sous le terme général de façonnés, par opposition aux unis, on englobe tous les tissus de soie - la variété en est grande - dans lesquels le jeu des fils, la combinaison et l'opposition de la chaine avec la trame, comme aussi la réunion d'armures différentes, figurent des dessins.

On dira que broder une étoffe, c'est également la façonner : rigoureusement, la chose n'est pas inexacte; il est fort supposable, au reste, que le travail lent et détaillé de l'aiguille, appliqué à un fond uni pour le rehausser, a chronologiquement précédé celui du métier, aussi bien qu'il a toujours, depuis, concouru avec ce dernier pour l'ornementation des tissus; mais, en fait, il y a aujourd'hui une démarcation qui s'est bien établie entre les deux genres et l'on fait une classe à part de la broderie sur soie, en dehors des soieries façonnées.

Ces dernières se présentent, à qui veut les étudier, à deux points de vue différents et toutefois inséparables : à celui, tout d’abord, des moyens matériels qui permettent leur fabrication, aujourd'hui très perfectionnée sous le rapport de la facilité et de la commodité et qui la rendent courante en beaucoup de cas; à un autre, ensuite, qui est celui du dessin, considéré en lui-même pour la valeur artistique de sa composition, l'habileté et le soin de sa préparation et de son établissement en vue de sa meilleure adaptation au tissu et de sa bonne exécution; toutes choses, en l'espèce, de première et si grande importance, qu'au risque d'être taxé de mettre la charrue devant les bœufs, il nous paraît mieux d'envisager la question du façonné sous cet angle en premier lieu, en insistant sur les difficultés d'ordre pratique que rencontre l'application ou mieux l'union d'un dessin au tissu par le tissage.

Ces difficultés viennent, pour une bonne part, des limites restreintes dans lesquelles l'artiste compositeur est enserré et qui lui sont imposées par les données, souvent étroites, de la fabrication. Elles peuvent être, pour le crayon le plus sûr, le pinceau le plus exercé, la palette la mieux nuancée et l'inspiration la plus fertile, une gêne trop réelle à laquelle il n'est pas commode de remédier sans une expérience approfondie.

" A la pureté du dessin », dit un maître réputé en l'art du tissage de la soie, Peyot, qui professait vers 1860 , « à une imagination féconde, nos artistes doivent joindre l'étude approfondie de la partie technique et pour ainsi dire matérielle de leur art ».

De ceci résulte une opposition entre le métier et l'art, un dualisme qui n'a jamais mieux été compensé évidemment qu'aux époques où la rapidité 
d'exécution, le coût limité des étoffes à produire n'étaient pas des conditions imposées, non plus que les goûts ne subissaient les prompts changements, les revirements de la Mode. C'est en usant de cette force du temps employé sans compter que les artisans chinois, persans, indiens, arabes, palermitains, etc., sont parvenus à exécuter les tissus à dessins dont l'art mérite constamment l'attention, aussi bien que peut étonner leur fabrication parfaite quand on songe aux moyens matériels dont ils disposaient pour cela, très primitifs en comparaison de ceux d'aujourd'hui.

On est en droit de supposer, qu'à ces époques lointaines, la division du travail n'existait pas, c'est-à-dire que l'office du dessinateur et celui du tisseur n'étaient pas choses distinctes; le savoir et l'habileté professionnelle de l'artisan devaient réunir ces deux spécialités d'aujourd'hui.

A quel moment en est-on venu à procéder autrement? Il serait bien difficile de le préciser, pas plus que de relever des noms d'auteurs pour les dessins des admirables soieries façonnées de la Renaissance italienne entre autres. Il faut en venir aux productions françaises du Xvil ${ }^{\mathrm{e}}$ siècle pour trouver des notions plus précises. On sait, en effet, que, dès lors et plus encore au siècle suivant, sous l'influence de peintres et de décorateurs célèbres, tels, par exemple, que Lebrun, Bérain, Baptiste Monnoyer, Oudry, Pillement, Boucher, Bachelier, et à l'imitation des compositions qu'ils inspiraient particulièrement pour les tapisseries des Gobelins et de Beauvais, se formèrent des dessinateurs lyonnais dont le talent, combiné avec une étude approfondie du tissage, décora un si grand nombre de belles et magnifiques soieries.

Citons Ringuet, Courtois, Reve1, Dacier, Bourne, Dutillieu, Bony, Berjon parmi les noms les plus remarquables de cette brillante pléiade, au premier rang de laquelle il faut placer, sans conteste, Philippe de Lassalle, qu'on a fort justement appelé ${ }^{1}$ le Raphaël du dessin de Soieries. Cet artiste incomparable est l'auteur de riches étoffes de tenture et d'ameublement exécutées, pour la plupart, en collaboration ou pour le compte du réputé fabricant Pernon, dont certaines ornèrent des résidences royales et impériales et que leur beauté a rendues célèbres à bon droit.

En étendant les proportions du dessin à des limites que l'on n'a pas dépassées, il sut en varier la composition, toujours réalisée avec le souci d'un ensemble sans défaut, en même temps obtenir la richesse et l'heureuse association des nuances et, en un mot, atteindre à la perfection, dans un style en parfait accord avec la recherche et le goût raffiné des productions artistiques de son époque et qu'il a marqué d'une maîtrise très personnelle; il fut, en même temps, l'inventeur de divers perfectionnements apportés aux métiers d'étoffes façonnées. Cependant, après trente années de sa vie, passées à ces tâches si dignes de lui assurer une vieillesse tranquille et à l'abri du besoin, Philippe de Lassalle termina son existence presque misérablement. La Révolution et le siège de Lyon l'avaient ruiné et il dut réclamer une aide de la Municipalité lyonnaise pour se voir débarrassé des soucis

${ }^{1}$ Les Dessinateurs de la Fabrique lyonnaise au XVIII siecle, par E. Leroudier, A. Rey, Lyon, I908. 
matériels les plus urgents; injustice du sort trop fréquente envers les artistes et les inventeurs, qui n'épargna pas non plus Bony, que son talent délicat et non sans force parfois place immédiatement après Philippe de Lassalle et Jacquard destiné, lui aussi, à finir dans la médiocrité.

Le rôle et les connaissances exigées du dessinateur pour les étoffes de soie ont été, on ne peut mieux, étudiés et précisés dans un ouvrage intéressant et

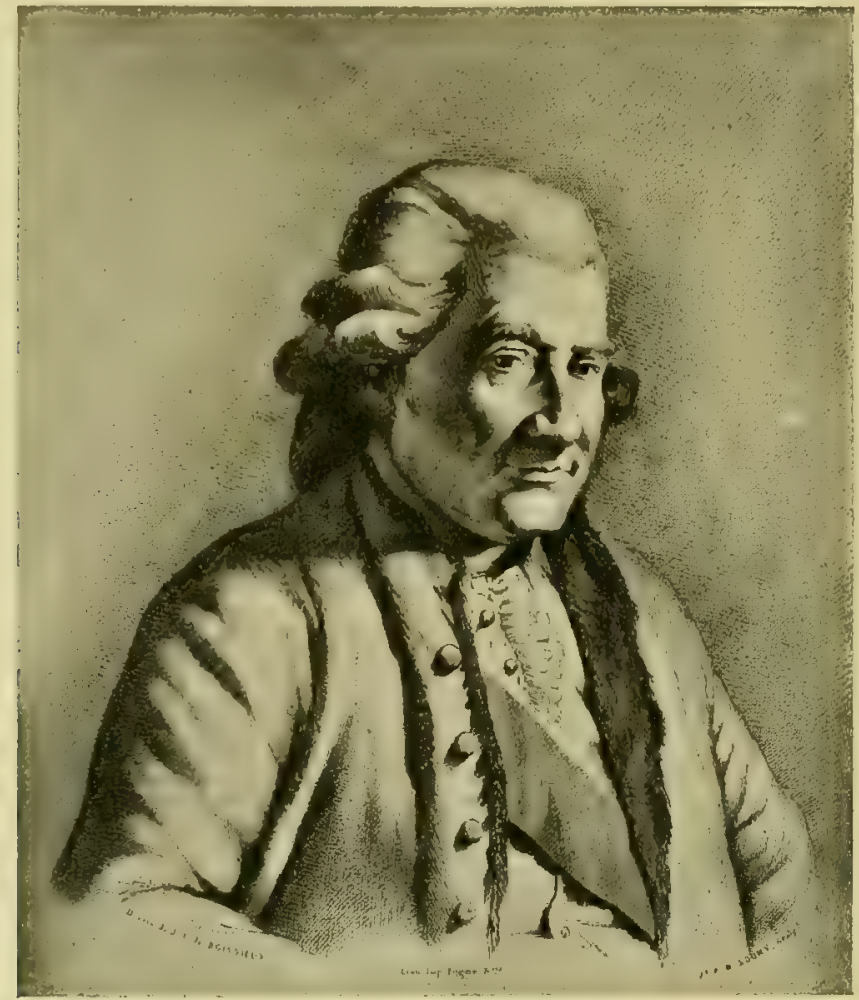

Philippe de Lassalle $(172 ;-1805)$.

(D'après l'estampe de J.-J. de Boissieu.)

assez rare publié en 1765 par l'un d'eux, Joubert de l'Hiberderie, qui exerçait à Lyon à ce moment et acquit lui-même une réputation justifiée; le titre en est: le Dessinateur pour les Fabriques d'Or, d'Argent et de Soie. C'est un pittoresque recueil de conseils éclairés donnés à des confrères par un confrère expérimenté, rompu aux finesses du métier et avisé des notions les plus générales comme de l'influence excellente sur l'imagination créatrice du dessinateur par mille choses diverses observées dans la nature, la vie mondaine, scientifique ou artistique.

Un chapitre détaillé et non moins attrayant est celui consacré au voyage à Paris, que l'auteur déclare étre, une fois l'an au moins, indispensable au dessinateur qui veut former son goût et. trouver des idées neuves; il devra 
visiter les galeries officielles de peinture, comme les collections particulières de tableaux et d'estampes, les manufactures royales des Gobelins, de la Savonnerie, de Sèvres, les châteaux et jardins Royaux, les monuments, la ménagerie et le jardin des plantes du Roi ; ne pas oublier les magasins à la mode d'étoffes et d'objets d'art les plus divers; enfin; chercher l'élégance partout où elle se montre, au théâtre et dans tous les endroits fréquentés par le beau monde d'une capitale, où le mouvement même, incessant et varié, des rues et des avenues, a de l'intérêt pour qui sait observer.

On ne saurait mieux dire aujourd'hui, ni peut-être mieux faire. Il est certain et assez curieux, que le goût des étoffes à dessins n'a pas augmenté, depuis Jacquard, en proportion des facilités apportées à leur tissage par son invention; de fait, les efforts des dessinateurs se sont trouvés peu à peu découragés à notre époque par la désaffection marquée de la Mode pour les étoffes façonnées de robes, sans que cela soit une raison, du reste, de la supposer définitive; on s'est vite déshabitué de voir les toilettes composées avec des soieries à fleurs ou à motifs, que l'on a remplacées par d'autres, unies, plus souples et tombantes, mieux adaptées au costume moderne, qui n'est point assujetti à un style défini et dont les formes, complexes et variables, sont régies par une fantaisie toute de saison.

D'autre part, l'absence de style s'était montrée dans les dessins depuis le milieu du $\mathrm{XIX}^{\mathrm{e}}$ siècle, après des temps comme ceux de la Restauration et de Louis-Philippe, où le goût n'avait pas brillé ; elle n'a fait que s'affirmer depuis lors. Avec du talent et de l'habileté professionnelle, on n'a le plus souvent, pour l'ameublement, fait que reproduire avec peu, ou quelquefois point de modifications, les dessins des siècles passés; quant aux dessins pour robes, ils ont témoigné d'un éclectisme très large. Tour à tour on a reproduit fréquemment, avec un succès qu'il faut reconnaitre, la fleur naturelle, débarrassée de toute donnée ou stylisation conventionnelle; puis l'ornement, tourné, retourné, combiné en mille fantaisies, on est allé jusqu'à l'emploi de formes imprévues, mélangées bizarrement, dessins valant par l'absence de dessin, dits "incohérents ». Enfin, souvent on a copié simplement encore de vieilles soies en s'inspirant de toutes les écoles et de tous les styles. La tendance de l" "Art nouveau " a donné lieu à des essais pour la décoration des soieries qui n'ont pas toujours prouvé, à quelques exceptions près, peut-être ', la facilité suffisante ni le bonheur d'adaptation du genre inspiré par cette formule aux tissus de soie.

Ainsi, jusqu'à présent, notre époque n’a pas mis son empreinte certaine, visible, dans les productions de l'art appliqué à la soie, non plus que dans d'autres branches de la décoration. Il serait déplacé ici de prétendre à démêler les causes du fait indéniable, aussi bien que d'entrer dans des considérations détaillées sur les caractères et la valeur comparée des styles relevés sur les tissus en général et les soieries en particulier à travers les âges et les civilisations; on trouvera ces études complètes dans les ouvrages spéciaux. Au moins est-il permis de se féliciter qu un nombre aussi rela-

1 On a exécuté, notamment, sur des modeles composés par Karbowski, Giraldon, Follot, certaines soieries d'ameublement, intéressantes ct décoratives. 
tivement grand de matériaux de tissus des époques anciennes nous aient été heureusement conservés.

Nous pouvons voir de la sorte ce que Byzance, Palerme, Venise, pour ne citer que ces manufactures, ont emprunté à l'Orient en continuant, en développant, en transformant peu à peu ses formules ornementales. Nous pouvons admirer tout ce que l'art italien du Moyen Age et de la Renaissance

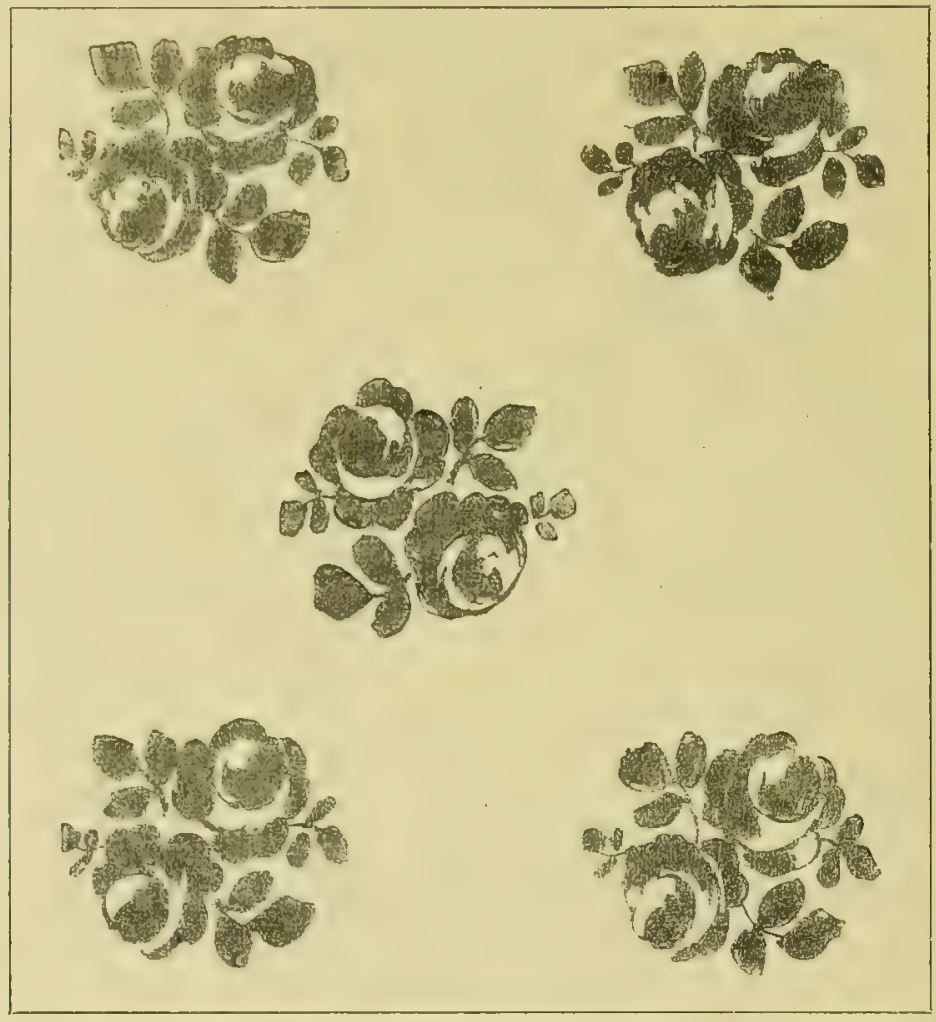

DESSIN SEMÉ CONTRESEMPLÉ !

a mis de beauté, de richesse, d'unité dans la composition, comme de variété dans le dètail, à des soieries justement réputées. Enfin, les productions françaises qui retracent à nos yeux aussi bien le faste somptueux et lourd du Grand Siècle que les grâces légères du XVIII", les charmantes mièvreries du Directoire et la décoration éclatante, guerrière, rénovée de l'antique du Premier Empire, contribuent à compléter cet ensemble de modèles si précieux.

Et sans doute, il n'en faut pas souhaiter, encore et toujours, l'imitation

1 Tous ces exemples sont dus à l'obligeante communication de M. George, à Paris. 
servile et constante, mais, à l'encontre de certains avis opposés, on peut croire très fermement que l'étude soutenue, la connaissance approfondie de ces monuments tissés, à côté de cette intelligente observation de tout, préconisée par Joubert de l'Hiberderie, est d'importance parmi les plus sérieux et utiles moyens de préparation pour l'artiste dessinateur qui veut

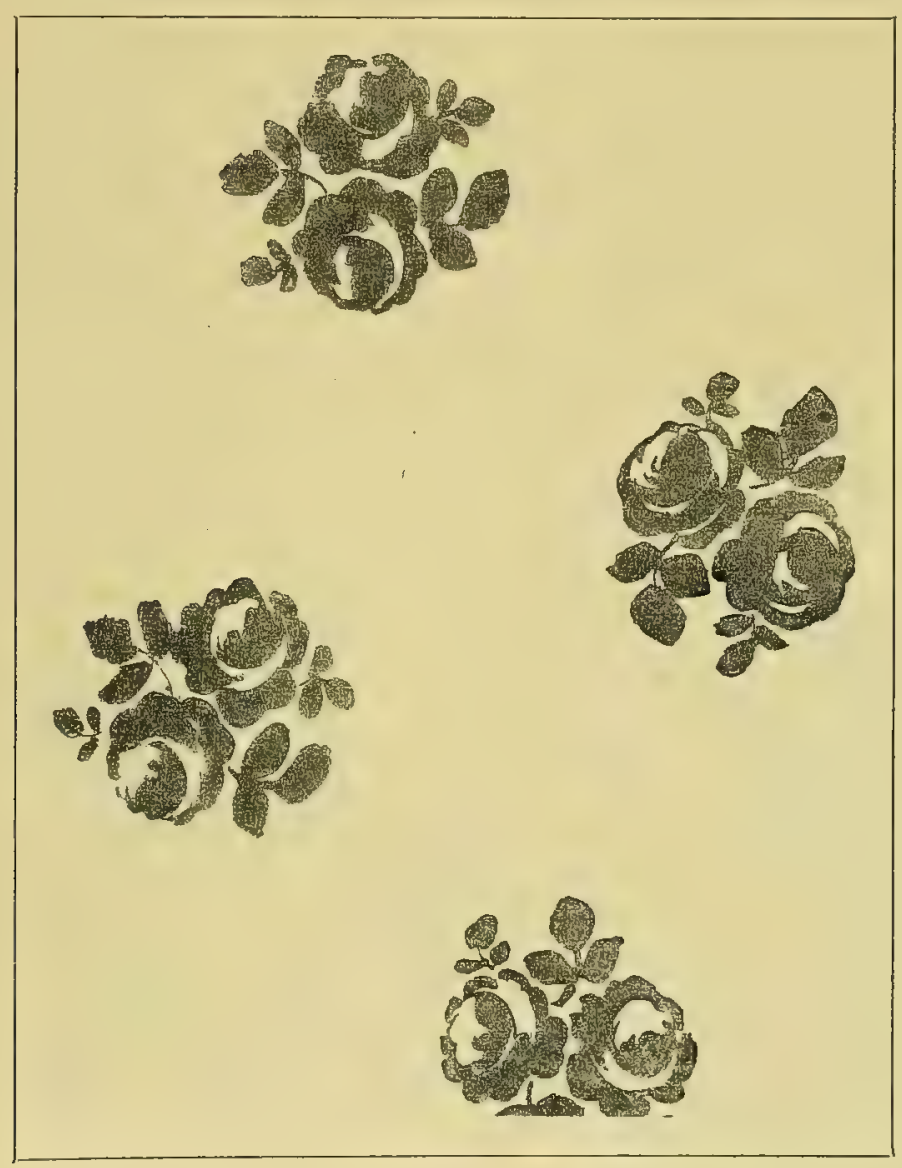

DESSIN SEMÉ SAUté.

créer à son tour; c'est comme une sorte de gymnastique salutaire et, mieux même, indispensable à la formation de son goût et de son expérience.

Voyons maintenant comment est préparé le dessin qu'on veut appliquer à l'étoffe. Le dessinateur en établit sur le papier l'esquisse en faisant quelquefois au préalable une pochade ou tracé hâtif, destiné à fixer rapidement le jeté d'une idée que son imagination ou que les indications du fabricant lui ont suggérée; l'esquisse est établie aux dimensions et proportions exactes que le dessin doit avoir au tissu.

A ce moment intervient l'importante question du rapport, dont les étoffes à rayures nous ont amené à parler déjà quelque peu. Comme on l'a 
remarqué, en effet, la plupart du temps le dessin ne se développe pas sur toute la largeur de l'étoffe, et cela pour deux raisons : la première, que les organes du métier commandant le jeu des fils et faisant que la chaîne se mêle à la trame en produisant à des places déterminées des points successifs et juxtaposés, assez comparables au travail de l'aiguille ou bien des armures différentes, ne peuvent pas pratiquement être multipliés assez pour correspondre au nombre très élevé des fils qui composent par milliers une étoffe de soie mème de qualité moyenne; la mécanique de Jacquard et celles plus perfectionnées, inventées à sa suite, n’ont pu fournir le moyen de réaliser - sauf cas spéciaux et arrangements particuliers - le fonctionnement isolé, individuel de chaque fil. Et ce que l'on a trouvé de plus commode pour parer à la difficulté a été de grouper un certain nombre de fils pour leur faire exécuter le même jeu, accrochés qu ils sont à la même commande; surtout, on a distribué ces fils dans des parties symétriques de la chaîne appelées chomins, parties qui sont de largeur prévue, calculée et dans chacune desquelles le dessin sc répète identiquement. Bien entendu, ces divisions, dont on use pour les besoins de la cause, ne se vérifient sur l'étoffe aux yeux attentifs que par la répétition du dessin. Or, cette répétition est imposée d'ailleurs en tous les cas où l'on se propose d'obtenir des dessins de peu d'importance, pour lesquels la largeur habituelle de l'étoffe est un champ trop vaste; deuxième raison qui, liée aux ingénieux moyens pratiques expliqués ci-dessus, limite en la plupart des cas l'esquisse à ne donner l'aspect que d'une partie de l'étoffe elle-même.

Le crayon du dessinateur doit donc se mouvoir dans les limites dune largeur indiquée pour chaque étoffe par le fabricant, largeur qui est celle du chemin lui-même, imposé par la disposition du métier et se trouve sousmultiple de la largeur totale. C'est ainsi que l'on dit constamment : dessin en $2,3,4,5,6,7,8$, etc., chemins, le dessin en I chemin étant le cas de celui qui occupe toute la largcur de l’étoffe. A moins encore que, par un artifice commode dont on voit à chaque instant l'application dans les soieries d'ameublement, le dessin ne soit composé de deux parties rigoureusement semblables, mais de sens opposé, ou « retournées » qui, juxtaposées au milieu du tissu, donnent lapparence d'une composition unique et régulière; c'est le dessin dit à retour s'appliquant au métier monté en 2 chemins, dont l'un suivi, le second à retour.

L'esquisse doit prévoir tous ces cas, ménager les raccords, c'est-à-dire éviter que la jonction des chemins ne se fasse d'une façon incorrecte, par suite de parties du dessin mal placées, et assurer ces raccords aussi bien dans la largeur que dans la hauteur ou longueur. On ne peut, en effet, avec le métier de façonné comme avec l'aiguille de la broderie, varier tout du long de la pièce les effets du dessin ; une fois qu'on l'a adopté et réglé toutes choses au métier pour l'obtenir, on est tributaire de ce même dessin qui se reproduit indéfiniment au fur et à mesure du travail. Encore faut-il que cette succesșion se produise d'une manière agréable à l'œil.

Insister sur ces détails, c'est montrer les soins attentifs qu'exige la préparation de l'esquisse et dont aucun ne peut être négligé, même pour le semé de pois le plus simple, à distances égales ou proportionnelles, les plus quelconques motifs ou bouquets alternés régulièrement en quinconce ou 
contre-semplé ou sauté et les dessins plus importants à ramagcs, guirlandes ou ornements courant tout au travers, dits aussi fonds pleins; cela sous peine d'une exécution mauvaise produisant des sortes de lignes ou de barres du plus fâcheux effet sur l'étoffe façonnée.

En un mot, toutes prescriptions qui sont à combiner avec le souci de l'élégance du dessin, aussi bien qu'avec les ressources limitées que chaque

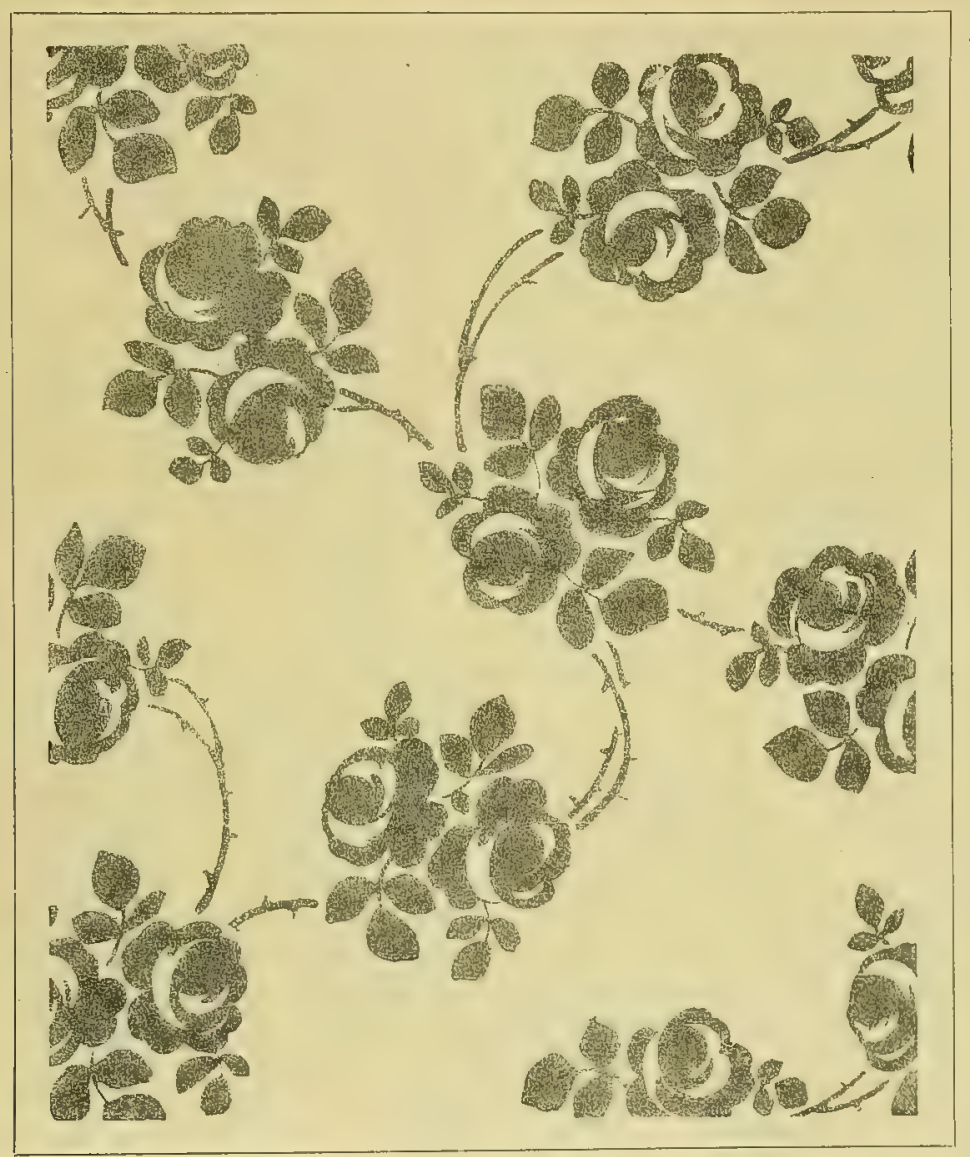

DESSIN A RAMAGES OU A FOND PLEIN.

genre de façonné comporte suivant l'entente particulière, avec lesquelles il faut arriver à ménager le meilleur coloris par des lumières et des ombres bien

1 Cette expression dérive de l'ancienne organisation du métier à la tire, comprenant un ensemble de cordelettes destinées à commander les mouvements des fils, lequel formait le semple. Lorsque, après avoir exécuté un dessin par la mancuvre successive des cordes choisies ou lacs, on répétait cette manœuvre dans l'ordre inverse, on reproduisait le dessin retourné à une autre place et on avait contre-semplé. Par analogie, lorsqu'un dessinateur dispose sur son esquisse des bouquets de fleurs alternés et retournés, on dit qu'ils sont contre-semplés. 

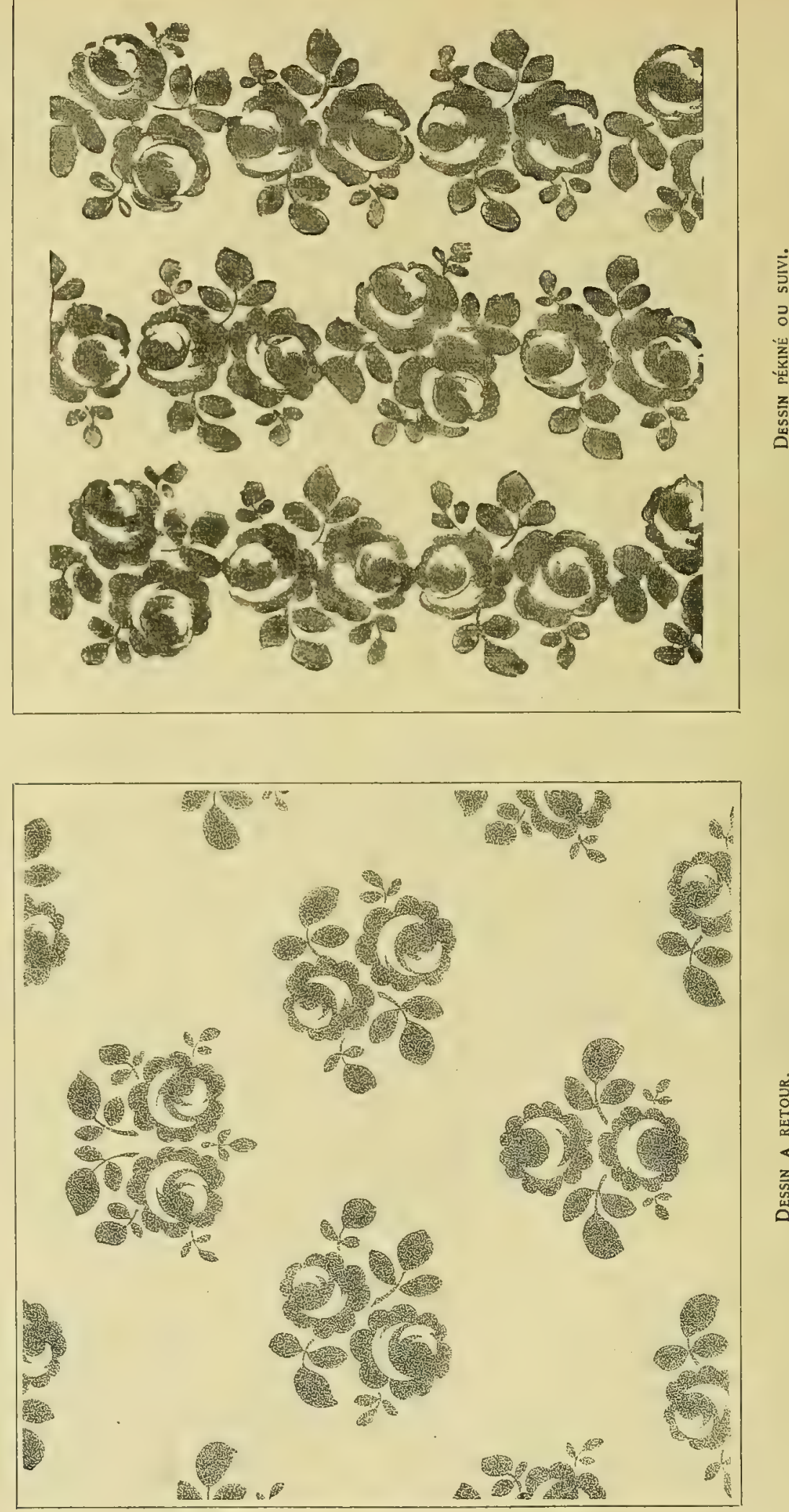

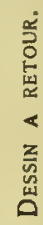


placées; c'est un ensemble méticuleux qu'on n'en finirait pas d'énumérer et l'on conçoit la restriction qu'elles apportent à l'imagination du compositeur.

Lorsque celui-ci a mené à bien son esquisse et qu'elle a été adoptée par le fabricant, alors intervient un autre professionnel auquel elle est remise pour être mise en carte. La mise en carte est la traduction du dessin en une sorte de langage de convention, traduction restée indispensable pour

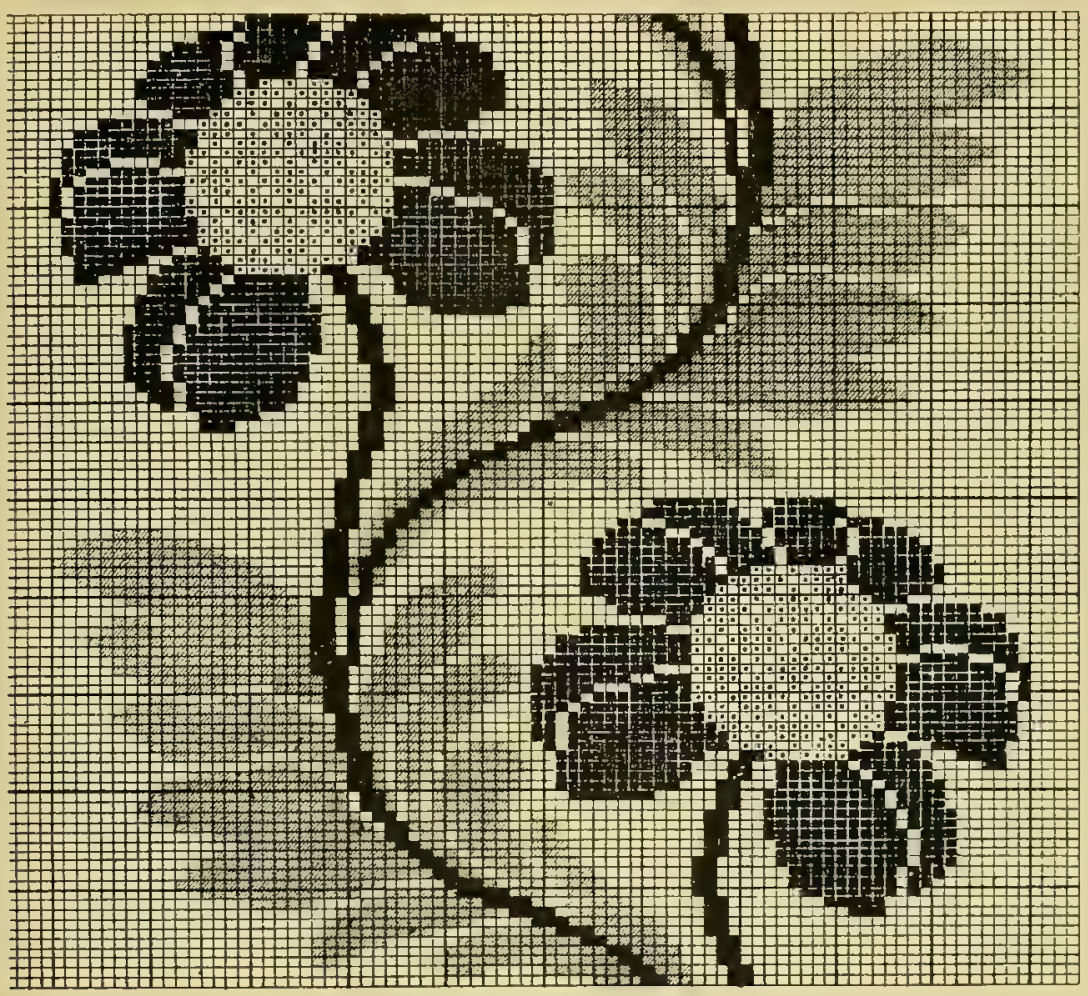

EXEMPLE DE MISE EN CARTE ${ }^{\prime}$

l'exécution au métier. Elle se fait sur un papier spécial, tout quadrillé à l'avance de fines raies perpendiculaires les unes aux autres; le dessin est reporté sur cette feuille à l'aide d'une ou de plusieurs couleurs de gouache franches, telles que vermillon, carmin, jaune, etc., qui ne signifient rien, du reste, par la hauteur de cette teinte même et son choix, mais bien par leur place; elles représentent, en effet, chacune un jeu déterminé de fils, un point ou une armure particulière, et, réparties, carreaux par carreaux, dans l'intervalle des lignes existant sur la feuille de mise en carte, qui correspondent conventionnellement aux fils eux-mêmes du tissu, elles règlent ainsi par avance le croisement de chacun avec la trame.

Etant entendu, par exemple, que le vermillon mis sur la carte représente

1 D'après Falcot. 
du taffetas et le jaune du satin, toutes les parties peintes à la carte en vermillon seront taffetas au tissu, celles peintes en jaune du satin et ainsi de suite.

Tout l'art du metteur en carte - résultat, on le conçoit, d'une longue pratique et de la connaissance parfaite de la fabrication - consiste donc non seulement à opérer ce report de l'esquisse sur la carte en respectant, autant que possible, la donnée générale, les lignes, les proportions du dessin, mais aussi à préciser, en connaissance de cause, fil par fil ou groupe de fils par groupe de fils, le jeu qu'ils devront exécuter pour produire les flottés, les

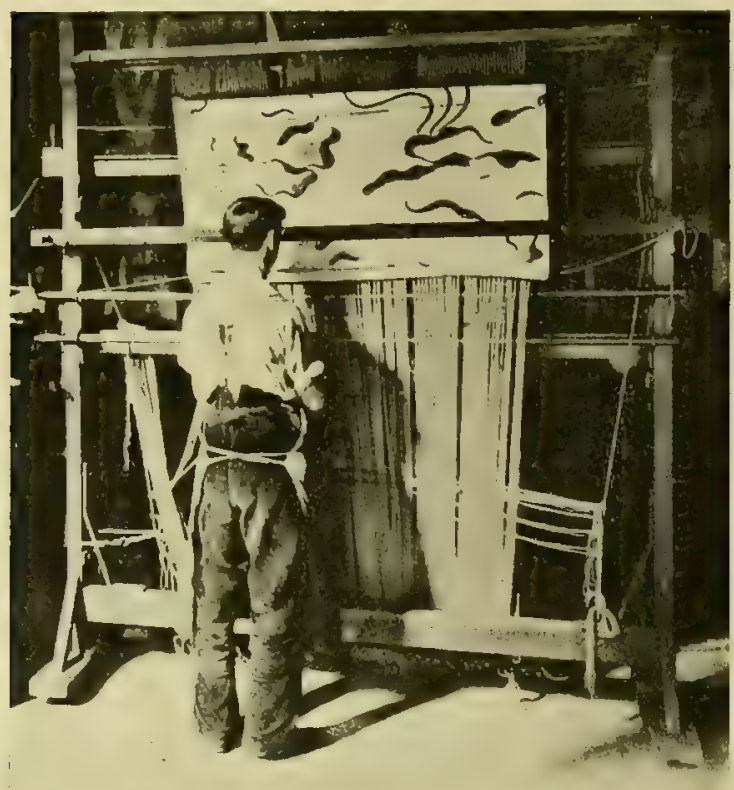

LISAGE DES DESSINS D'APRÈS LA MISE EN CARTE ${ }^{1}$. armures dont l'ensemble combiné formera finalement, ren$d r a$ le dessin sur l'étoffe.

La figure ci-contre, reproduisant une mise en carte, quoique ne pouvant donner que l'effet du noir avec le blanc, facilitera la compréhension du procédé; on y voit que les lignes courbes s'expriment par une suite de petits carreaux garnis de couleur en décochant - c'est le terme employé - les uns sur les autres, tout comme les fils le feront au tissu par découpures plus ou moins fines; les effets ombrés se rendent par les mélanges de différents points dits fondus, berclés, taquetés, etc., etc.

La carte une fois terminée et revue avec soin, pour vérifier que tout est bien prévu pour une bonne exécution du tissage - placement correct des trames pour l'endroit et l'envers, travail compensé des fils, etc. - il reste à la lire. C'est une opération certainement compliquée qui n'est pas non plus très simple à rapporter. Une description détaillée des organes du lisagc, aussi bien que de ceux de la mécanique Jacquard et du métier de façonné, serait assez inutile dans ce but, même avec le secours de nombreuses figures et d'explications minutieuses; il faut voir fonctionner ces appareils sous ses yeux pour en saisir l’ingénieux système.

Bornons-nous à dire que lire une carte - travail qui est confié à des ouvriers expérimentés et souvent à des femmes - c'est choisir sur le semple du lisage, ensemble de cordelettes verticalement disposées en nombre et proportion conformes aux données de la carte, celles d'entre elles qui, prises ainsi et entrelacées avec d'autres cordelettes transversales et successivement placées comme la trame doit l'être dans le tissu, détermineront, quand on

1 Clichis P. Martel, à Lyon. 
les tirera une à une, le mouvement d'un emporte-pièce venant se placer dans le logement présenté par une plaque métallique ad hoc; la série des emporte-pièce ainsi choisis produira, lors de l'opération subséquente du piquage, le percement de trous aux endroits convenables de chacun des cartons de la mécanique.

Ces cartons, liés les uns à la suite des autres et en ordre bien entendu, se

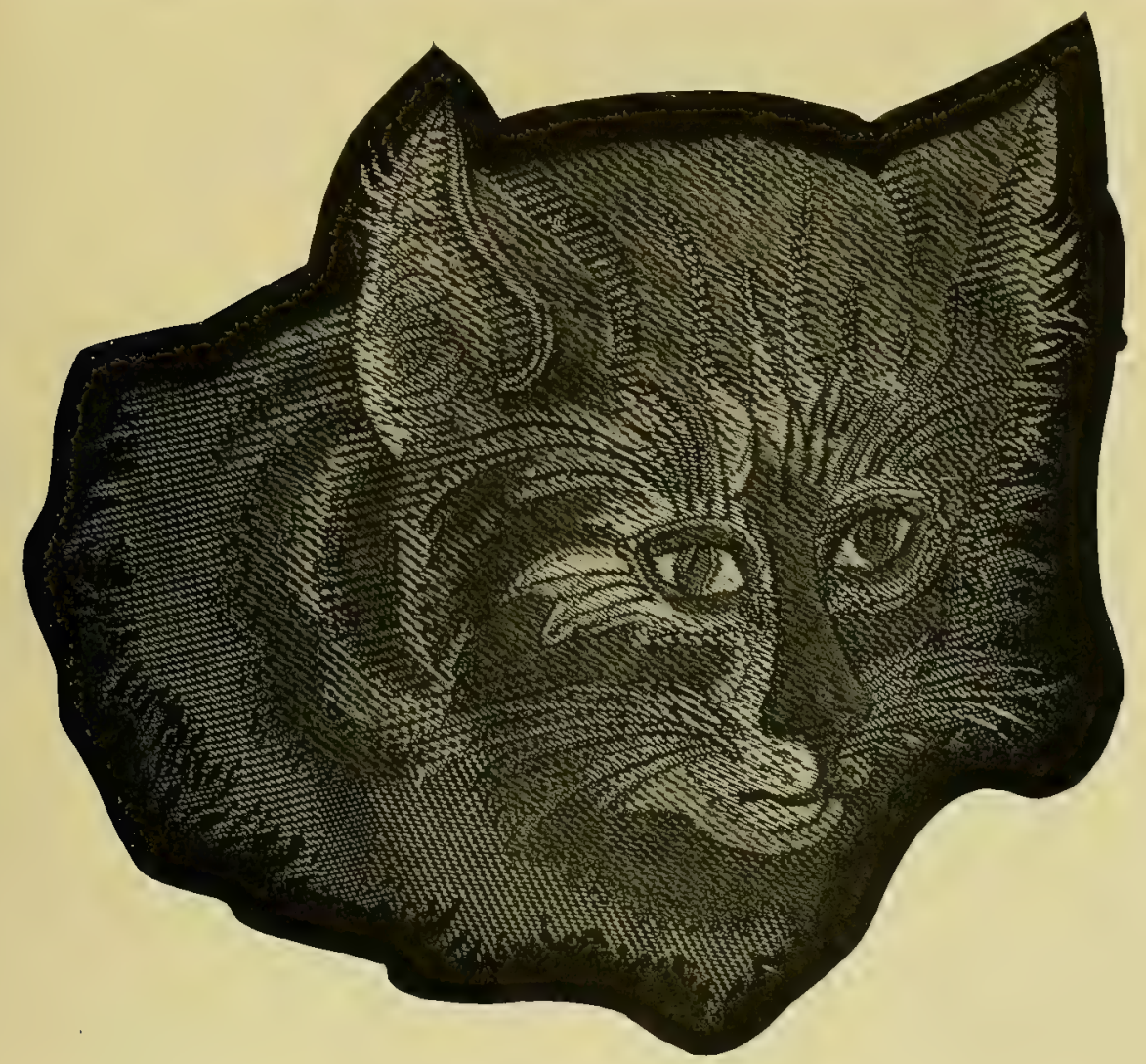

EXEMPLE DE SOIERIE FACONNÉE

MONTRANT LE MODELÉ OBTENU JAR LES DIFFÉRENTS POINTS DE TRAMES.

présenteront un à un dans la mécanique au fur et à mesure de la marche du métier, en appuyant contre un ensemble de tiges métalliques dites aiguilles. Lorsque ces aiguilles rencontreront des trous dans le carton, elles passeront et détermineront, par le mouvement d'un crochet dont chacune est solidaire, la levée diun ou de plusieurs fils de la chaîne. Un groupe de fils levant ainsi tous ensemble ou masse, pendant que les voisins à droite et à gauche restent en fond, c'est-à-dire non levés, il se produira, la trame une fois passée et retenue dans le tissu, un point ou flotté. Ainsi de suite et de proche en proche et de complications en complications dans ces manouvres et commandes successives de fils, ayant pour résultat final le façonnage de l'étoffe de cent manières différentes. 
Tel est, très succinctement résumé, le principe fécond dont s'est inspiré Jacquard pour réaliser son invention. Avant lui, on usait, pour les étoffes à dessins, du métier à la tirc, dont l'emploi fut immémorial pour ainsi dire et dans lequel l'ensemble des cordes ou semple du lisage était placé sur l'un des côtés du métier lui-même et correspondait, par des poulies de

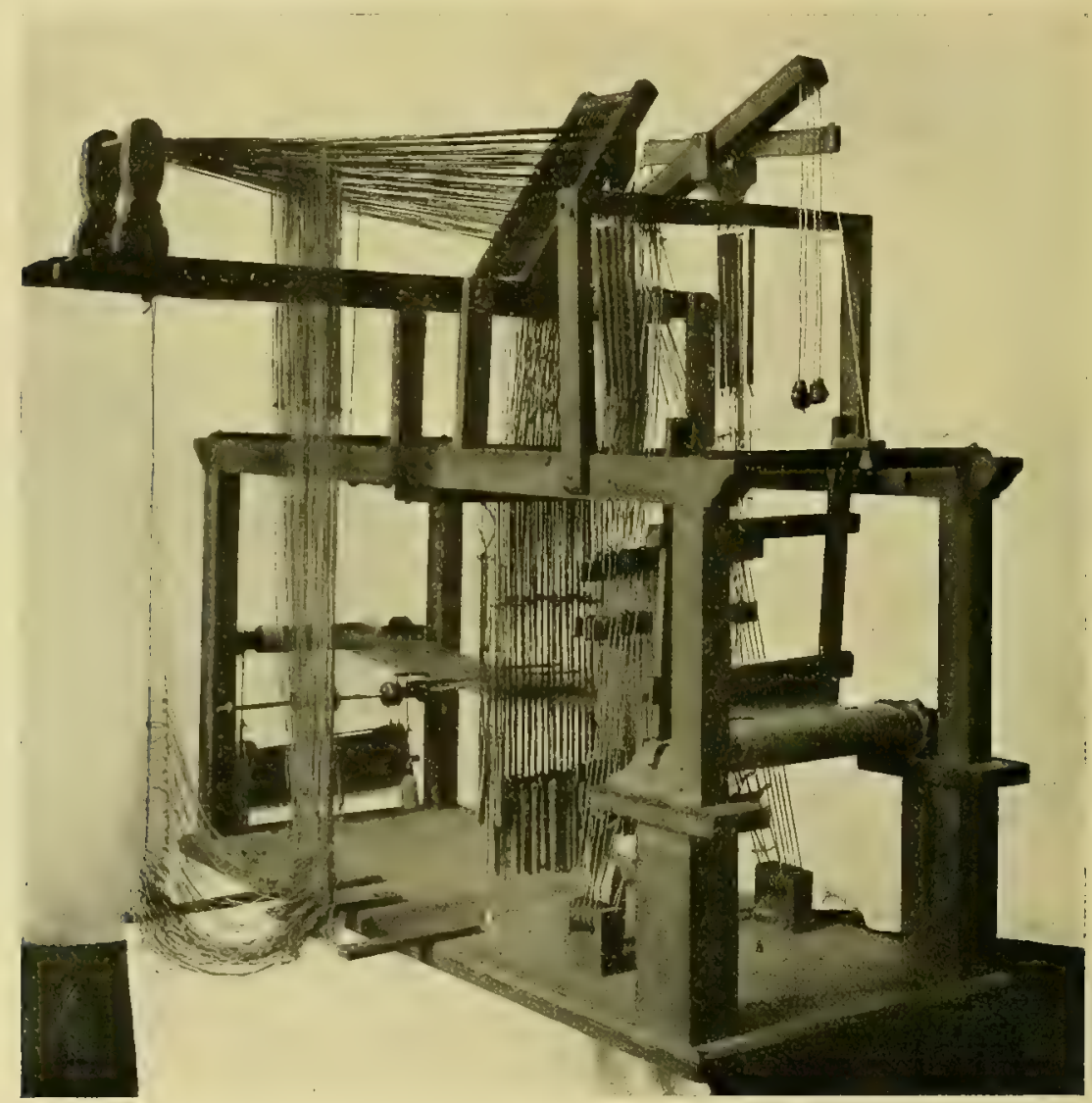

MODELE D'ANCIEN MÉTIER A la TIRE.

renvoi et des arrangements particuliers, à la commande des fils; 1e dessin se lisait à chaque coup de trame, au fur et à mesure du tissage, mais deux ouvriers étaient nécessaires pour le travail : l'un, le tireur de lacs (cordes entrelacées) s'occupait de cette préparation du dessin et de la mancuvre des fils de chaîne, et lautre, le tisseur, passait la trame et la mettait en place avec le battant et le peigne. Le travail était long, difficile et coûteux. Il en fut ainsi cependant pendant bien longtemps, malgré les perfectionnements apportés entre autres par Dangon ou Dagon, inventeur à Lyon, en I606, du métier à la grande tire; par Galantier, Blache, Bouchon ; par Falcon, créateur, en 1728 , du métier dit à la Falconne, et aussi par Philippe 
de Lassalle, désireux de réaliser avec la plus grande perfection et le plus de commodité ses grandes compositions.

Cependant, un sieur Régnier, de Nìmes, fit fonctionner vers la fin du XVIII ${ }^{\circ}$ siècle, paraît-il, un métier à cylindres commandant le dessin, qui supprimait l'un des ouvriers, le tireur de lacs, avec un succès sans doute très relatif, car on ne voit guère la chose citée que pour mémoire.

Enfin, l'illustre Vaucanson, préoccupé à différents titres du travail des manufactures de soie, dont il fut un des inspecteurs et pour lesquelles il avait perfectionné les moulins à tordre ou organsiner la soie, construisit, entre autres inventions, un métier à cylindres également, qu'un seul ouvrier devait suffire à faire mouvoir, si l'on s'en rapporte à une notice parue dans $l c$ Mercure de France de novembre 1745 et plus louangeuse que bien précise. Le modèle de ce métier est au Conservatoire des Arts et Métiers, à Paris, et ses organes, longtemps dispersés, furent, paraît-i1, rassemblés et remontés par les soins de Jacquard lorsqu'il vint à Paris, en $\mathrm{I} 804$, bien après la construction de sa propre mécanique qu'il avait fait breveter en I80I. Mais ce n'avait été, du reste, entre les nombreux travaux du célèbre mécanicien des automates fameux, qu'une sorte d'expérience de laboratoire; il avait répudié, dit-on, cette œuvre de ses mains, négligé d'en établir le plan et la description comme pour ses autres œuvres.

A Jacquard revient donc le mérite entier de l'idée de sa mécanique. Né

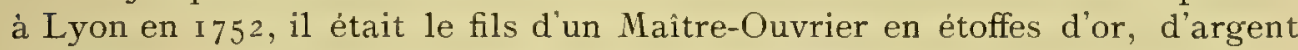
et de soie et, de bonne heure, bien qu'il eût débuté par apprendre la profession de relieur, il avait songé à des perfectionnements qui simplifieraient la marche des métiers qu'il voyait fonctionner de façon difficile et fatigante. Contrarié dans ces recherches de son esprit inventif par les embarras procurés par ses moyens d'existence précaires, il connut encore les jours sombres du siège de Lyon sous la Révolution et, après avoir servi comme volontaire sur le Rhin dans les armées de la République, c'est à grand'peine, à son retour dans sa ville natale qui se relevait lentement de ses ruines, qu'il obtint de quelques amis les concours pécuniaires indispensables pour la construction de la machine dont il avait, depuis longtemps, le projet et pour sa présentation à l'exposition des produits de l'industrie nationale de I $80 \mathrm{I}$.

La récompense qui lui fut accordée n'était cependant que la dernière médaille de bronze de celles décernées. Néanmoins, cette invention avait eu du retentissement à l'étranger. Et la Municipalité de Lyon commença, comme témoignage de l'intérêt porté à ce compatriote, par le loger au Palais des Beaux-Arts et, peu après, à propos de l'invention d'une autre machine à fabriquer les filets de pêche maritime, Jacquard se vit mandé à Paris par le Premier Consul et installé au Conservatoire des Arts et Métiers.

Il semblait que la destinée de l'homme qui était resté toujours infiniment modeste dans ses goûts et sans aucune ambition démesurée fùt enfin orientée vers ses travaux favoris, dans le calme assuré et avec des honneurs mérités. Il étudiait les procédés employés à la Manufacture des Gobelins et $\mathrm{y}$ apportait d'heureuses modifications, lorsque, sur des sollicitations pressantes, il revint à Lyon; un atelier lui était fourni en mème temps qu'on lui confiait un enseignement industriel. A pareille époque, un décret impérial fixait son existence en autorisant l'Administration Municipale de Lyon à 
lui accorder une pension viagère de trois mille francs. On assure même que Napoléon, en signant le décret, dit : «En voilà un qui se contente de peu! 》

Et cependant les désillusions et les déboires allaient encore assaillir fortement l'inventeur. On sait qu'il rencontra la plus vive résistance auprès des fabricants lyonnais pour l'adoption de sa mécanique, que ceux-ci prétendirent être sans valeur réelle ni pratique; les ouvriers ne se montrèrent pas moins réfractaires, excités et prévenus qu'ils avaient été à l'avance contre un

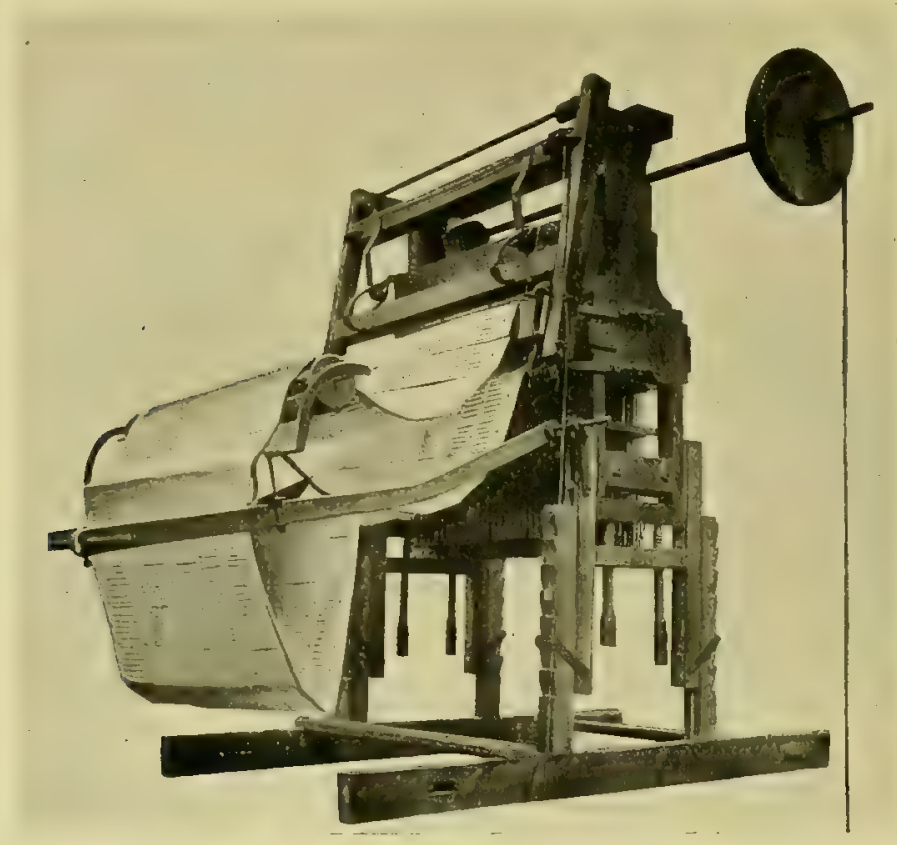

Mécanivue Jacquard.

système qui, supprimant une partie de la main-d'œuvre, devait à leur compte les conduire à la misère!

On sait aussi quaprès avoir supporté les jalousies, les tracasseries les plus mesquines, les injustices les plus criantes, Jacquard eut la douloureuse tristesse de voir, un jour, le métier modèle qu'il avait construit et qui était déposé au Palais des Beaux-Arts, enlevé par une populace ameutée et brûlé sur une place publique de Lyon.

Et pourtant il ne perdit pas courage; sans se lasser, il entreprit à nouveau de convaincre ses concitoyens, aveugles volontaires, et de se faire rendre justice; il y parvint enfin et, une fois sa machine adoptée, se retira du monde et des affaires pour vivre paisiblement de sa modique pension dans une retraite dont il ne sortit plus et où il finit ses jours en 1834 dans le dénuement presque le plus complet. L'amende honorable que lui avait faite l'industrie française et lyonnaise, la croix de la Légion d'honneur, une célébrité universelle, furent pour lui des compensations plus précieuses aux tribu- 


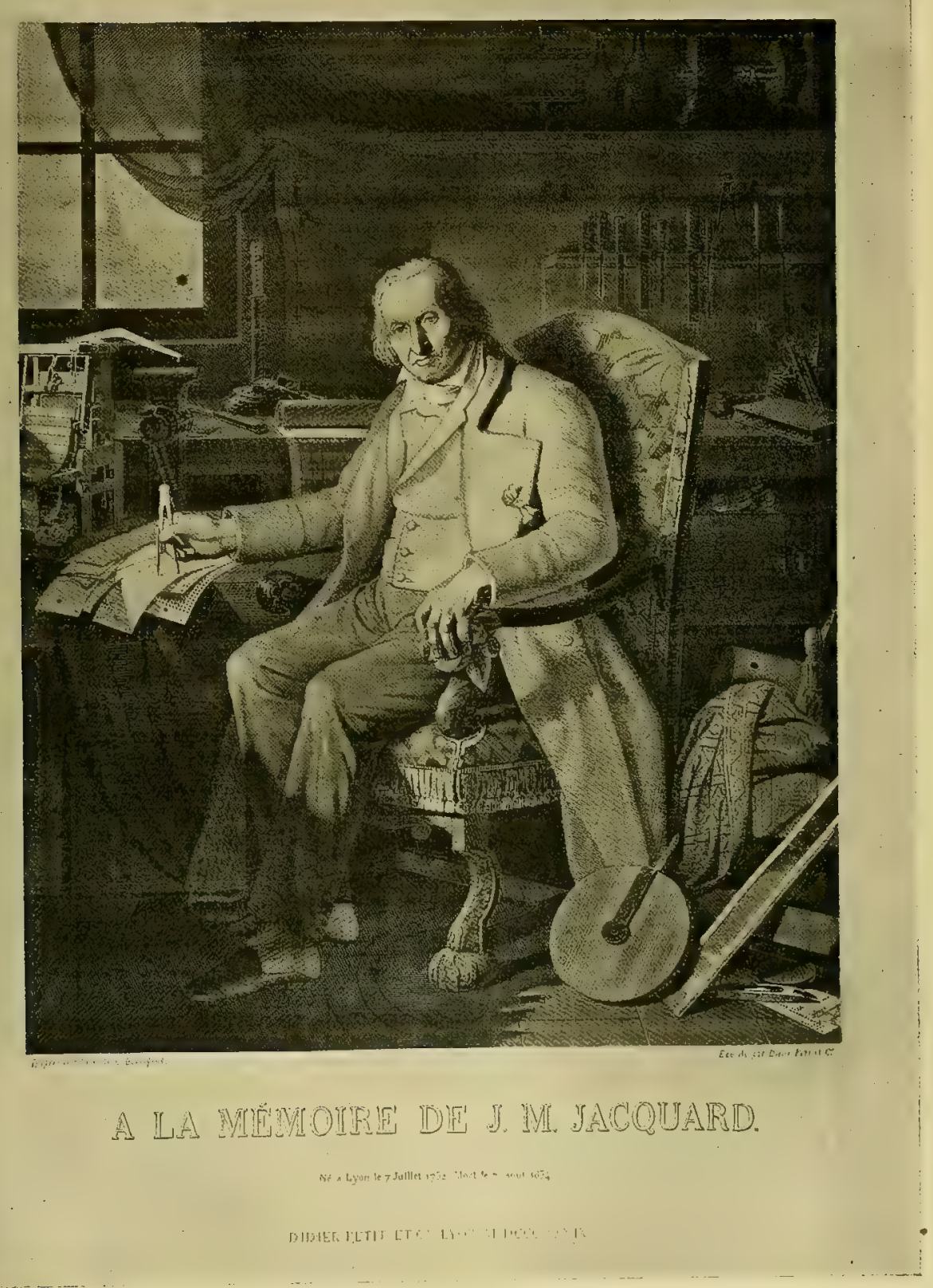

Portrait de Jaceuard, tissé en sole Par Didier-Petit et Cie, Lyon, 1839. (D'après le tableau de Bonnefond.) 
lations supportées que la richesse qu'il n'avait pas recherchée et qu'il tenait en médiocre estime. Tel fut 1'homme auquel des statues élevées à Lyon et à Calais sont des hommages de reconnaissance trop justement mérités.

Peu à peu des perfectionnements importants furent apportés à la mécanique telle que l'avait imaginée Jacquard; d'autres, plus complexes, ont été construites après la sienne et notamment en utilisant le papier perforé sans fin pour remplacer l'ensemble des cartons forcément lourds et encombrants, mais le principe est resté le même dans ces appareils nouveaux, aussi bien que la suite des opérations dont nous venons de donner une idée très abrégée ; on peut, en effet, se figurer que l'organisation, le montage des métiers destinés à fabriquer des soieries à dessins tissés, offre une très grande complication et une non moins grande diversité de procédés suivant le but poursuivi.

Un organe important du métier est le régulateur, inventé par Dutillieu, dessinateur et fabricant lyonnais, pour faire que le dessin se reproduise dans les étoffes de meubles toujours dans les mêmes proportions, ce qui assure un raccordement exact des divers lés d'une tenture.

Et combien de patients et habiles canuts, dont les noms sont restés discrètement dans l'ombre, ne se sont-ils pas ingéniés, à l'exemple de leurs ainés, maîtres et artisans d"autrefois, à trouver telles ou telles heureuses modifications dans larrangement des organes du métier, tels ou tels trucs subtils de tissage, et se sont montrés par là encore de précieux collaborateurs des fabricants et des dessinateurs pour la solution de délicats problèmes.

Il aurait paru impossible, il y a quarante ans, de faire fonctionner mécaniquement des métiers fabriquant certaines soieries façonnées et 1'on y est très bien parvenu cependant; beaucoup, néanmoins, de ces tissus ne peuvent encore saccommoder de ce genre de fabrication et réclament le travail à bras, infiniment plus souple et maniable. Mais il ne faut jurer de rien toutefois, et ne pas trop faire de réserves pour l'avenir. Déjà, pour quelques essais isolés et limités à la valeur de simples expériences, on s'est préoccupé d'utiliser la photographie pour simplifier et traiter la mise en carte par une exécution presque automatique, et l’électricité pour faire mouvoir les fils et le métier lui-même.

Peut-être sera-t-il possible de voir, plus tôt qu'on ne le pense, fabriquer des soieries façonnées et les plus riches avec des organismes ayant toute la précision rigoureuse de mécanismes purs et simples, supprimant ou remplaçant complètement la partie du travail du tissage, consciemment, intelligemment faite encore à la main.

Faudra-t-il alors se féliciter de ce succès définitif, total du machinisme dans l'art textile et dans sa branche la plus relevée, ou bien au contraire le déplorer pour des raisons diverses, comme celles, entre autres, toutes de sentiment, si l'on veut, de la disparition forcée et regrettable des derniers vestiges de traditions séculaires? Les avis seront certainement partagés et mieux vaut ne pas chercher de réponse à la question; en attendant, force est bien de laisser à la Mode, cette « Reine et Empérière du Monde », comme le disait Montaigne, le soin de décider par ses préférences souveraines du rôle que peuvent jouer les soieries à décor dans la confection d'élégantes et riches toilettes et pour l'ornementation d'intérieurs luxueux.

Il convient maintenant d'étudier de plus près quelques types envisagés 
comme les principaux parmi la très considérable variété qui existe de soieries façonnées; les difficultés d'un classement très net, d'une délimitation précise,

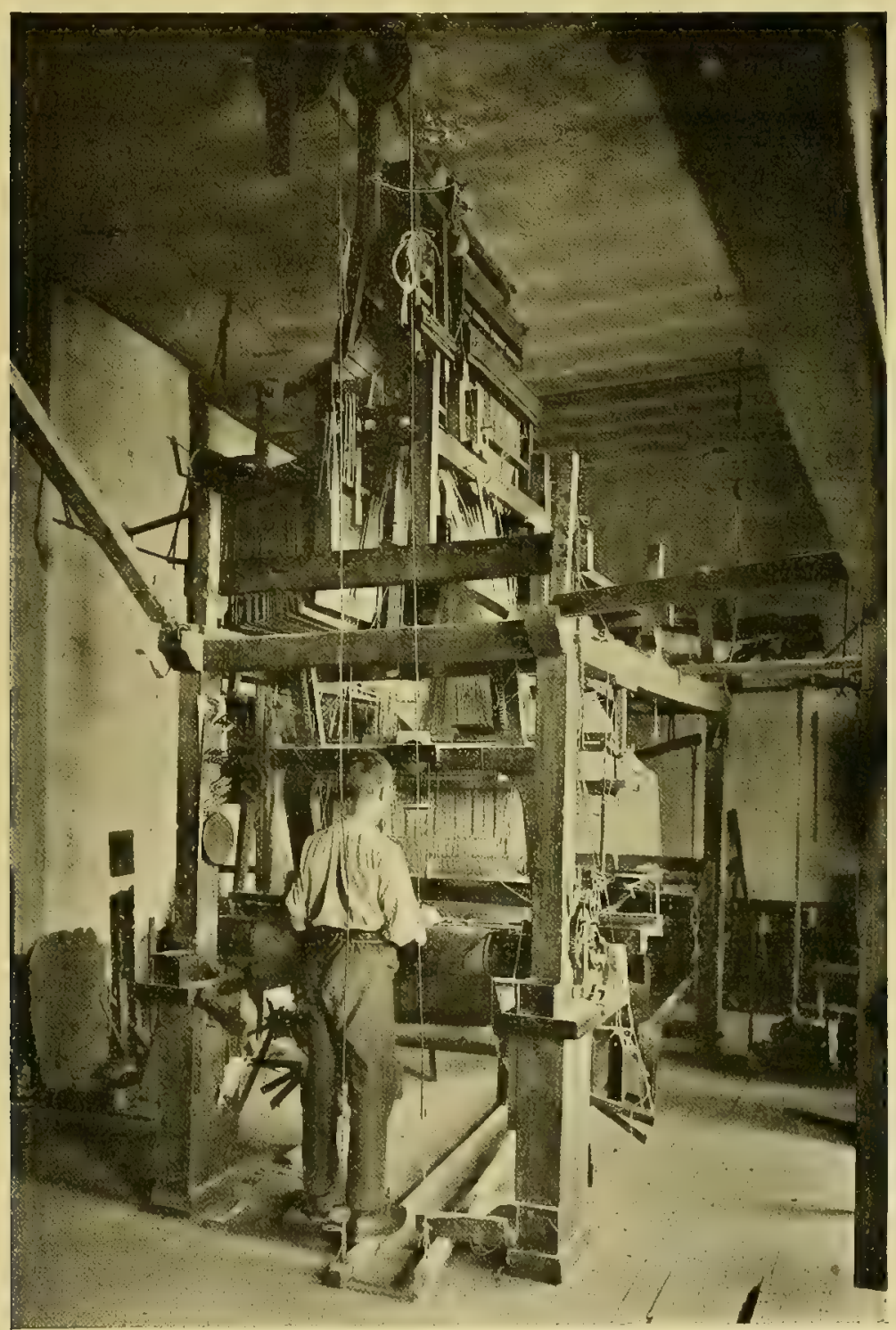

MÉTIEK A BRAS POUR SOIERIES FAÇONNÉES!

se retrouvent ici comme ailleurs; ce sont à peu près toutes les soieries unies qui peuvent être prises comme fond ou support de dessins formés par des

1 MM. Tassinari et Chatel, à Lyon. 
jeux de fils, trame ou chaîne, ou les deux ensemble; on façonne de la sorte taffetas, satins, serges, armures diverses, gazes, crêpes, velours, etc. ; de même également qu'on peut employer ces divers genres en mélanges l'un avec lautre; tout ceci, soit d'après des exemples et des types connus, soit

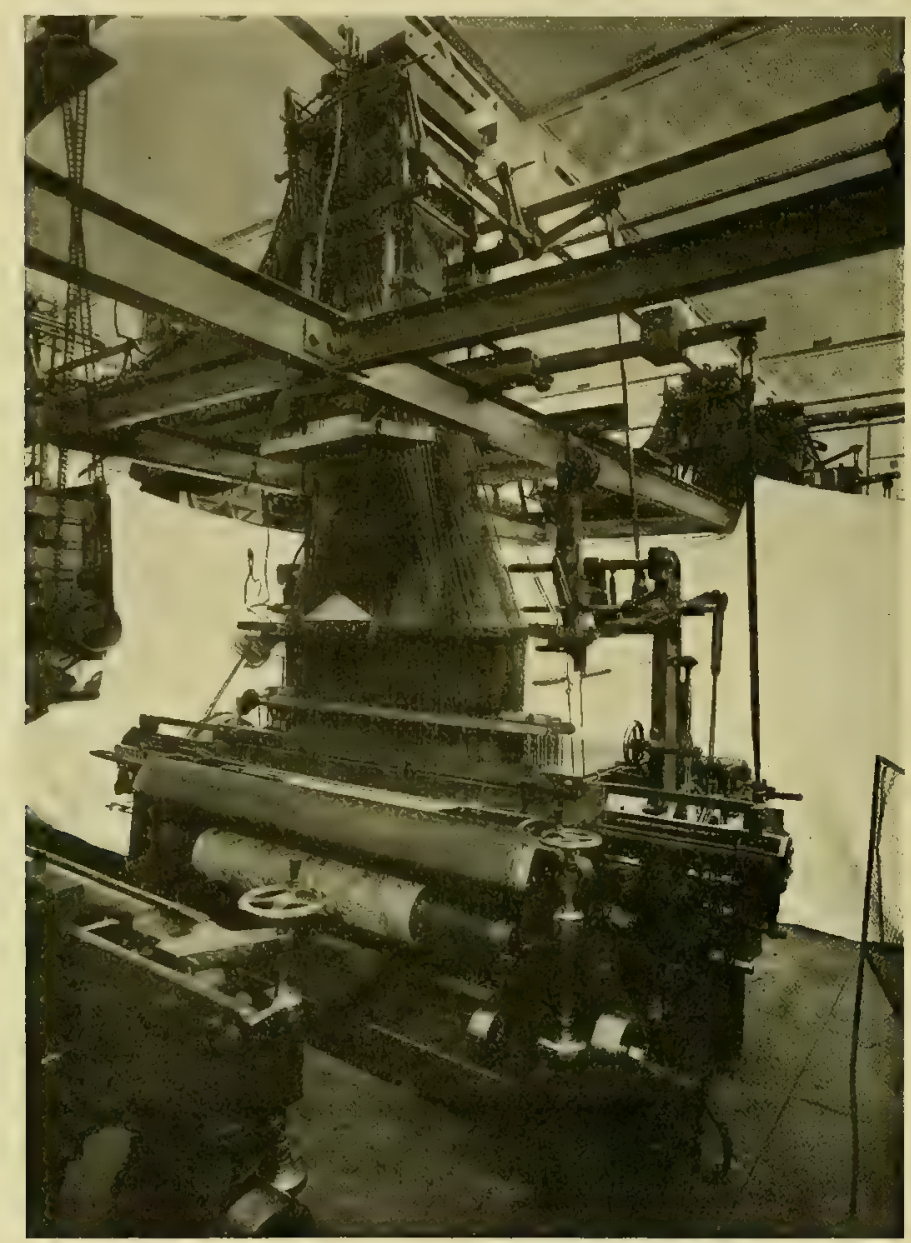

MétIer mécanique pour soleries façonnées 1.

par la recherche de combinaisons pour lesquelles l'imagination créatrice n'est arrêtée - et elle l'est souvent - que par les difficultés d'ordre pratique.

Dans toute cette vaste famille d'étoffes, famille chaque jour grandissante, nous allons choisir, sans nous réclamer d'un ordre méthodique, les plus connues ou les plus remarquables, celles qui nous paraissent constituer de véritables genres par leurs caractères assez bien définis.

1 M. Henry Bertrand, Lyon. 


\section{DAMAS. BROCATELLE ET LAMPAS}

Le Damas est sans doute la plus connue des différentes sortes de soieries à dessins. Cependant si ce nom damas éveille ordinairement et de prime abord l'idée de l'étoffe solide, résistante, de nuance uniforme et fort souvent d'un beau rouge cramoisi, au décor contrasté d'effets mats et brillants, sans endroit ni envers, il ne faut pas oublier que le terme est plus étendu et ne s'applique pas seulement à cette étoffe dont on fit tant de rideaux et couvrit tant de sièges; il y a damas et damas, présentant entre eux des différences appréciables :

damas pour ameublement, qui offre les caractéristiques ci-dessus rappelées, soit l'entente du damassé, si largement utilisée dans le linge de table et qui est un mélange de satin et de gros de Tours, formant tissu réversible, utilisable sur chaque face;

damas d'une contexture assez analogue mais mieux adaptée aux
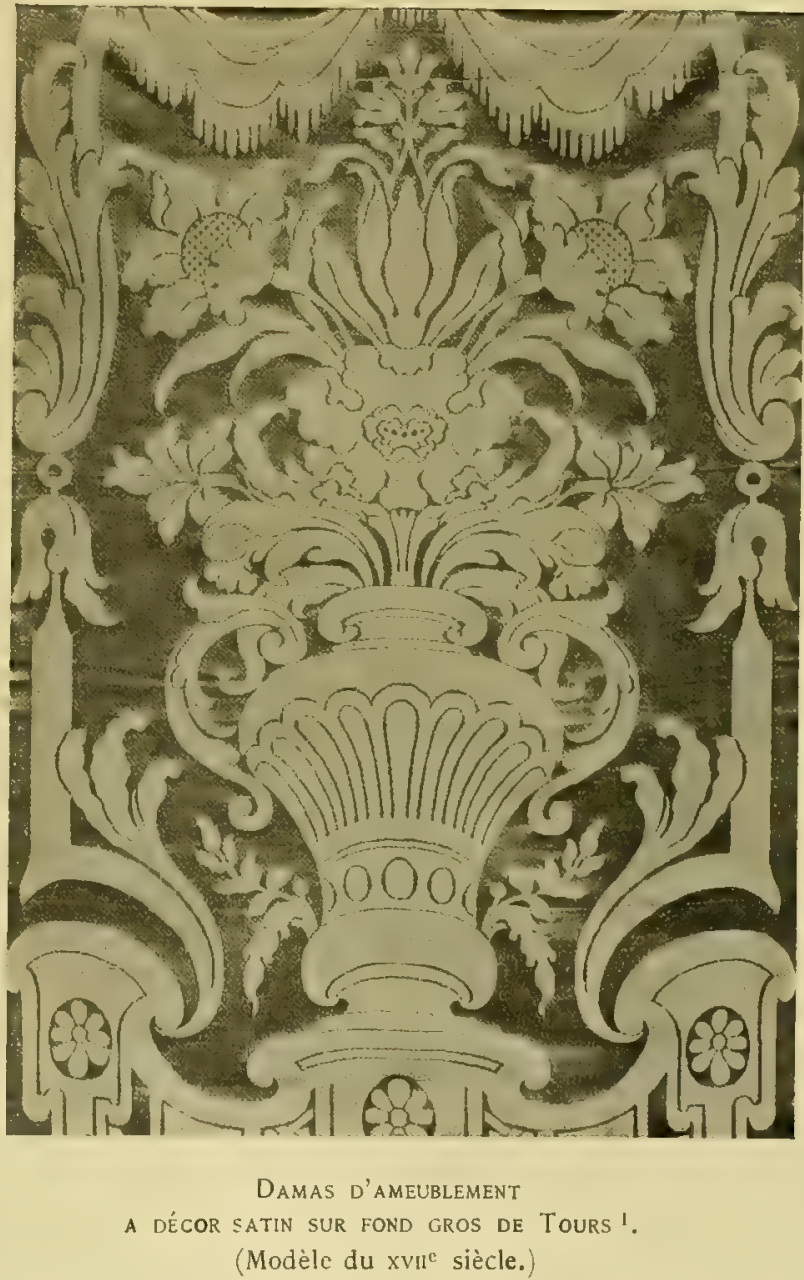

Damas d'AMEUbLEMENT

(Modèle du XvII ${ }^{\complement}$ siècle.) usages de la robe, dans lequel les armures satin et gros grain, prises l'une ou l'autre pour fond du dessin, se rehaussent presque toujours d'effets de trames, dits flottés ou lisérés. C'est le damas décoré de fleurs ou d'ornements divers qui eut, en noir surtout, un succès si grand et si longtemps soutenu pour confectionner des toilettes très délaissées maintenant; compris 
au goût du jour, on l'emploie presque plus pour le dessous des toilettes et leur doublure, que pour le dessus. Avec plus de recherche et de complication dans le jeu des fils, on a fait aussi des damas dont les effets imitent ceux de la broderie et qui en portent le nom ;

damas non plus faits seulement de soies d'une teinte unique ou nuance pure, mais variés par la combinaison d'une chaîne diune couleur avec une trame d'une autre ou bien même de plusieurs trames pour augmenter les ressources de la coloration; damas lamés ou brochés d'or et d'argent, et bien d'autres damas encore.

Et cette multiplicité ne date pas d'hier; elle existait déjà au Moyen Age lorsque les draps de Damas, ou plus simplement les damas, étaient apportés de la ville de Syrie, ou leur fabrication fut particulièrement florissante et dont ils devaient conserver le nom, qui était appliqué du reste, en même temps, à des objets très divers, céramiques, armes, etc., de la même provenance.

A cette époque, où ces tissus furent extrêmement recherchés et d'après un auteur érudit ": "Grande était la variété de ces précieux tissus que l'on trouve à chaque pas dans les anciens comptes. Les moins communs paraissent avoir été les damas blancs, qu'un poète du xivi siècle considère comme destinés à la Royauté, et les plus usités, les damas noirs. Quelquefois le dessin était à petit ourrage, comme les trois pièces qu'en i í6 Philippe le Bon commanda de livrer à Philippe Jossequin, garde de ses joyaux, et qui valaient vingt cinq écus d'or pièce. Il y avait encore des damas vermeils, verts, bleus, violets, cramoisis, des jaunes, des rouges, des gris, des damas fleur de pêcher et sans doute de bien d'autres nuances. On en vendait aussi de deux couleurs comme bleu et blanc, vert et violet, mais les plus chers. étaient ceux brochés d'or et d'argent. Certains étaient noirs, brochés avec des feuilles d'argent et figurés de soie verte ; dautres, décorés de losanges et d'oiseaux avec le champ rouge; d'autres enfin, de losanges et à lettres. »

Comme on le roit, ce n'était pas l'assortiment, ainsi qu'on le dit aujourd'hui, qui faisait défaut à cette collection de damas de toutes sortes, employés principalement alors à la confection de vètements, tant pour les hommes que pour les femmes; on englobait donc déjà sous une désignation unique des tissus assez différents les uns des autres et point toujours strictement damassés; de plus, ils ne provenaient pas uniquement de Damas mais de tout l'Orient.

309719

La Chine avait fabriqué ces genres d'étoffes de soie depuis les temps les plus reculés, les Indes également; Constantinople et l'Espagne en produisirent en bon nombre et, dès le $\mathrm{XV}^{c}$ siècle, les ateliers italiens de Florence, de Gènes, de Tenise, surtout de Lucques, en fabriquèrent et en exportèrent en quantité ; bientôt les damas de Lyon allaient obtenir à leur tour une fort grande renommée et la partager un temps avec ceux fabriqués à Tours.

Mais, comme on l'a dit, le " siècle d'or » du Damas - du Damas, étoffe d'ameublement - fut en France le XVII siècle; à cette époque fastueuse, cette belle et bonne étoffe façonnée de dessins de fleurs, de rinceaux, etc., fut l'ornement des demeures royales comme de celles des ministres et des

1 F. Michel, Recherches sur les Etoffes de Soie, II, $2 \mathrm{I}_{5}$. 
grands personnages, et parut même jusque chez les simples conseillers au Parlement. Louis XIV — on le voit dans les comptes du Garde Meuble Royal - fit constamment donner d'importantes commandes à ses fournisseurs attitrés de Lyon, de Tours, et même de Paris, de damas, qui étaient pour la plupart de ce rouge cramoisi qui est demeuré comme la teinte traditionnelle et officielle pour ce tissu.

Par contre, le damas jaune fut le plus en honneur sous Louis XV et employé également par quantité vers la fin du XviII ${ }^{c}$ siècle, moment où l'on rechercha encore les damas à deux et trois couleurs présentant le plus souvent des bandes et des dessins d'ordonnance droite.

Pendant le Premier Empire enfin les damas devaient jouer de même un rôle important dans l'ameublement. Les documents récemment retrouvés au Mobilier National et publiés par M. Dumonthier ${ }^{\perp}$ ont montré, de façon tout à fait intéressante, quelles quantités relativement considérables de ces étoffes furent exécutées à Lyon parmi les différentes commandes de soieries ordonnées par l'empereur Napoléon $I^{\text {er }}$, à destination de ses Palais. Dans le nombre de ces soieries plus ou moins belles et ornées, et pour justifier le programme de la «magnnificence alliée à la plus stricte économie », il se trouve même des damas qu'on appela économiques; pour un coût assez raisonnable alors, ils servirent à décorer les appartements secondaires des impériales résidences, tout en leur donnant cette apparence de richesse qu'il fallait pour assurer l'accord de ces dépendances avec tout le reste.

Le succès du damas de soie devait amener des imitations faites avec des matières textiles moins coûteuses et tout au moins des mélanges. Fort anciennement déjà, et à l'exemple des satins, il y eut des damas caffarts ou capharts, dans lesquels la chaîne était de soie et la trame du fil, de fleuret, de laine, de coton.

Plus tard, Abbeville et Caux fabriquèrent des damas d'ameublement fil et coton. En Hollande, on imita les beaux damas de soie avec d'autres faits de la même matière mais beaucoup plus légers. Enfin, on s'avisa de faire complètement avec de la laine ce genre de tissu aussi bien qu'en mêlant la laine à la soie et la laine au coton. Cette sorte de soie secondaire, faite de déchets, la schappe, que nous avons citée à maintes reprises, a été également fort employée dans le même but. Il est à remarquer enfin que le genre damas, de soie pure ou de soie mélangée, se réussit très bien aujourd'hui en teint en pièces, avec toutes les nombreuses commodités pratiques apportées par cette manière de procéder : tissage en écru d'abord, teinture et apprêt du tissu ensuite.

La Brocatelle est encore un damas, une sorte de damas dans laquelle le dessin est formé par du satin qui s'enlève assez en relief sur un fond fait d'une trame liée d'après une armure régulière et, le plus souvent, par du sergé. Cette trame, qui peut être de la même nuance que le satin ou dune teinte opposée, est supplémentaire à la trame qui sert à former le corps, le fond du tissu, qui est généralement, celle-ci, de fil ou de coton; on appelle lancé ce coup de trame supplémentaire; pour l'ajouter, en effet, l'incorporer au tissu,

1 E. Dumonthier, Etoffes dimeublement d'époque napoléonienne, Paris, Igog. 
l'ouvrier se servait autrefois d'une navette exclusivement maniée, lancée à la main, et, dans certains cas, ceci nécessitait le concours d'un aide ou apprenti appelé lanceur.

Il est bon de dire, à cette occasion, que le lancé est, somme toute, une sorte de cas particulier du tissage lorsquil comporte plusieurs trames, qu'elles soient, ces trames, ajoutées supplémentairement à celle du fond ou

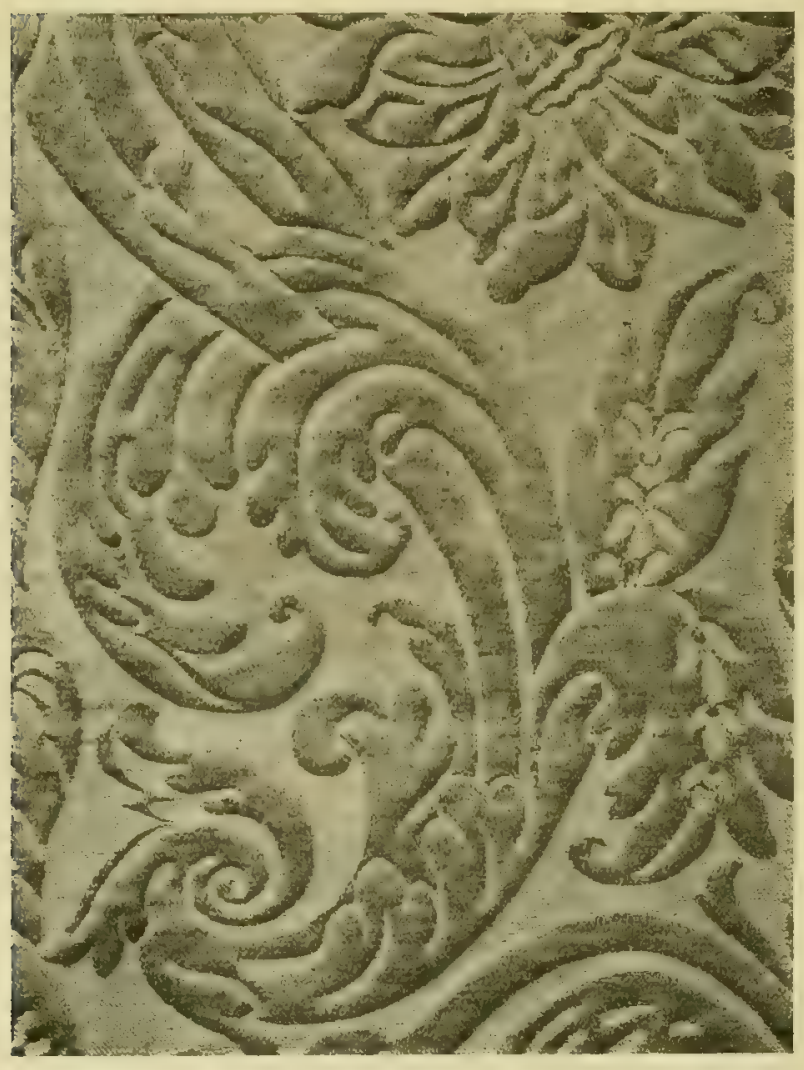

Brocatelle !. bien combinées avec celleci selon des artifices et des règles trop compliquées pour que nous tentions de les décrire. Tout ceci afin de constituer les étoffes dites à plusieurs lats, l'expression lat, qui revient à chaque instant dans l'étude technique des soieries à décor, voulant dire trame, comme on le voit; c'est ainsi qu'il y a des soieries façonnées à deux lats, à trois lats, à lats suivis, à lats interrompus, etc.

Pour en revenir à la brocatelle, qui est employée de nos jours dans l'ameublement sous la forme décrite plus haut, il paraît certain que ce nom ou plutôt les formes archaíques: brocardel, brocadel ou brocatille, correspondaient autrefois à des étoffes assez différentes de la brocatelle actuelle, assez diverses et, pour certaines, faites à l'imitation du brocart. Le fil de lin, la laine, le coton entraient dans leur composition avec la soie.

Parmi les brocatelles de soie à ramages qui simportaient en France d'Italie, celles de Venise étaient les plus estimées; après l'inventaire des Meubles de la Couronne et celui du Cardinal de Mazarin, la brocatelle de Venise figure dans celui de Molière ( 1673 ), celui du savant Costar, du maréchal d'Humières ( $168+$ ) et chez l'abbé d'Effiat.

On voit aussi que le nom de brocatelle, en mème temps que ceux de ligature et de mézeline, désigna des étoffes assez communes et de peu de valeur, ornées de carreaux ou de dessins à fleurs, que l'on fabriqua en Nor-

1 Communiqué par MM. Tassinari et Chatel. 
mandie et en Flandre et qui servaient à des tours de lits de campagne, à des tapisseries, tentures, etc.

D'après les mêmes principes de fabrication que ceux usités pour les

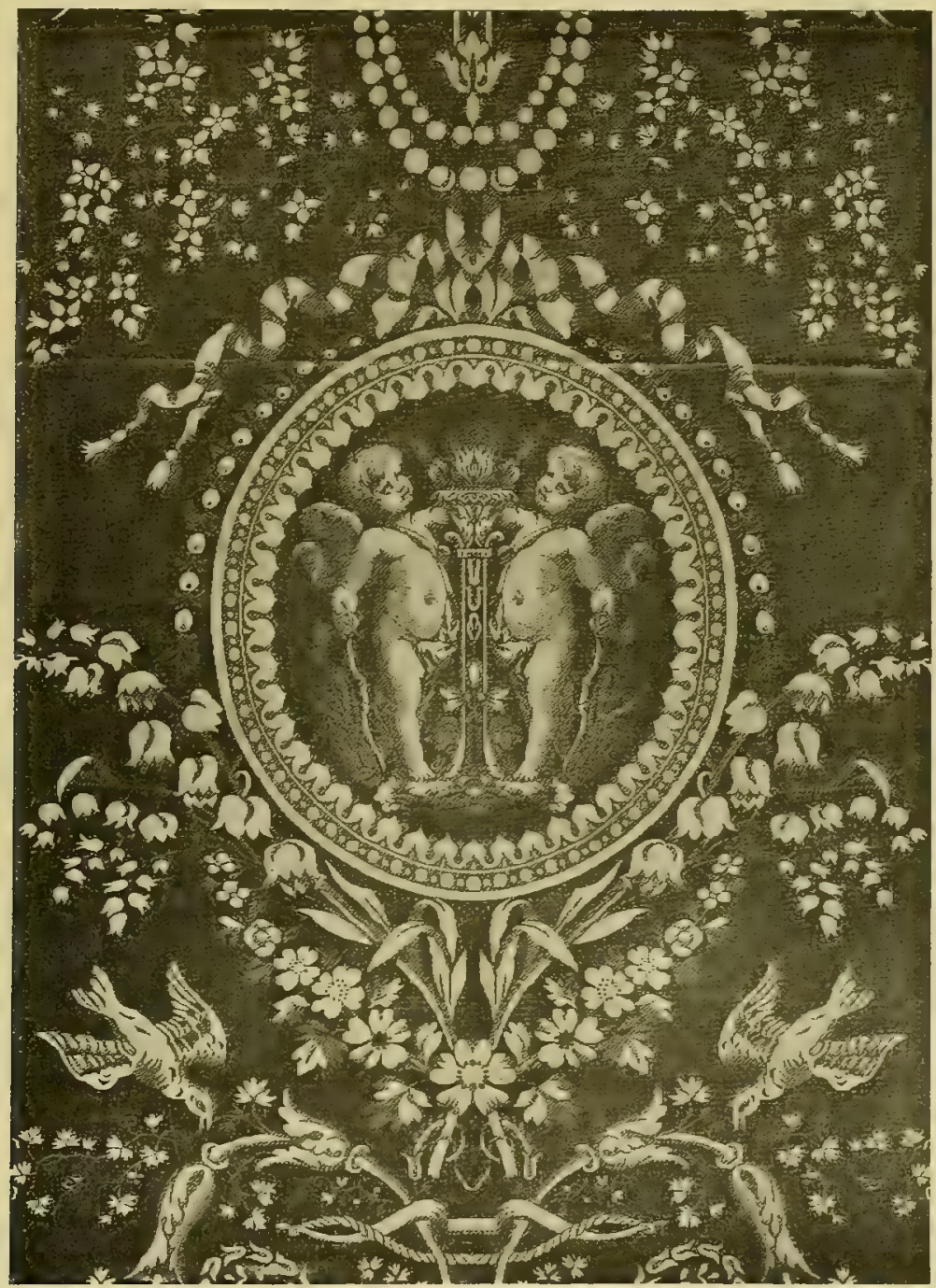

LAMPAS ${ }^{1}$. Modèle du XVII" siècle.

brocatelles d'ameublement, on a fait, à leur imitation, des brocatelles plus légères destinées à la robe, tout soie, et d’autres mélangées de laine, pour des gilets par exemple.

Comment définir de façon bien précise le Lampas? Ce nom, sans origine

1 Communiqué par MM. Tassinari et Chatel. 
bien précisée, a été appliqué, sans doute, à des tissus présentant plus ou moins de ressemblance entre eux; cependant, il est frère ou tout au moins cousin germain du damas, ce lampas d'ameublement qui se répandit en France vers la fin du XVIII siècle pour couvrir des sièges, faire des rideaux, des tentures; on lillustra de dessins aux rinceaux légers, élégamment fleuris et combinés avec des figures, des attributs, des ornements, et le château de Versailles lui-mème reçut des ameublements couverts de lampas bleu et blanc, comme ceux du plus charmant effet, dont beaucoup ont été conservés jusqu'à nous et sont encore reproduits tout pareils pour des emplois actuels.

Sous cette forme classique, le lampas est une soierie fond satin, sur lequel le dessin se détache, figuré par le jeu des trames de couleurs opposées qui en tracent les contours par des flottés, des lisérés; en plus, du taffetas que l'on peut obtenir pur, c'est-à-dire d'une seule nuance, opposée également à celle du fond, permet de varier les effets et, par suite, le décor général.

Sur des bases analogues, avec des recherches d'exécution plus poussées, on a fabriqué des lampas comportant certainement une ornementation plus riche et l'on a réalisé, à des époques récentes, de magnifiques étoffes auxquelles on a donné ce nom, soit pour en faire des robes de prix, soit pour en orner des intérieurs luxueux. Comment, d'ailleurs, pourrait-on affirmer que ces étoffes, dans leur beauté et leur difficulté d'exécution, aient bien tout le caractère de l'inédit et dépassent de beaucoup celles que l'on fit autrefois? Un exemple très curieux de ces rénovations, si fréquentes en cet ordre de choses, est là pour rendre plus prudent.

Lorsque, en 1817 , M. Bancel, de Saint-Chamond, en 1820 , M. Beauvais, de Lyon, en 1835 , MII. Grangier frères, de Saint-Chamond, eurent pris, de la meilleure foi du monde, des brevets pour la fabrication de diverses étoffes, on s'aperçut, un peu plus tard, qu'il y avait toutefois des antériorités respectables et plus qu'incontestables, puisqu'elles remontaient au début du Moyen Age! En effet, des échantillons d'étoffes tout à fait similaires existaient entre les feuillets d'un très intéressant manuscrit paléographique connu sous le nom de Manuscrit de Théodulphe, précieusement conservé dans le trésor de la cathédrale du Puy. Théodulphe ou son relieur les y avait fixés soigneusement pour préserver les lettres ornées d'or et d'argent de cette sorte de bible manuscrite, enrichie de poésies et de dissertations sur les Ecritures Saintes, offerte vers les dernières années du règne de Charlemagne, en accomplissement d'un vou, à Notre-Dame du Puy-en-Velay. Ces tissus avaient été choisis, sans doute, parmi les plus beaux de l'époque à laquelle vivait l'auteur du manuscrit; à leur attribuer, comme on a été en droit de le supposer après examen attentif, une origine orientale, pour les uns chinoise, pour les autres gréco-syrienne ou arabe, on voit quelles pouvaient être l'habileté et les connaissances des artisans ingénieux qui furent les précurseurs des nôtres de plusieurs siècles. Et, d'autre part, on connaît un autre manuscrit moins ancien que le précédent, qui appartient aux religieux de la GrandeChartreuse et dont la couverture était composée de plateaux d'ivoire réunis par un dos formé d'un «Lampas des Indes » argent et soie, tissu de travail tout à fait remarquable. Force est donc de conclure, après d'autres indications concordantes, à l'origine exotique et lointaine du lampas; il en est pour les choses de la soie comme pour tout le reste: rien de nouveau sous le soleil! 


\section{SOIERIES BROCHÉES}

Sans aller plus loin, il importe de dire quelques mots des soieries qui sont brochées, en précisant leur mode de fabrication. Une tendance très

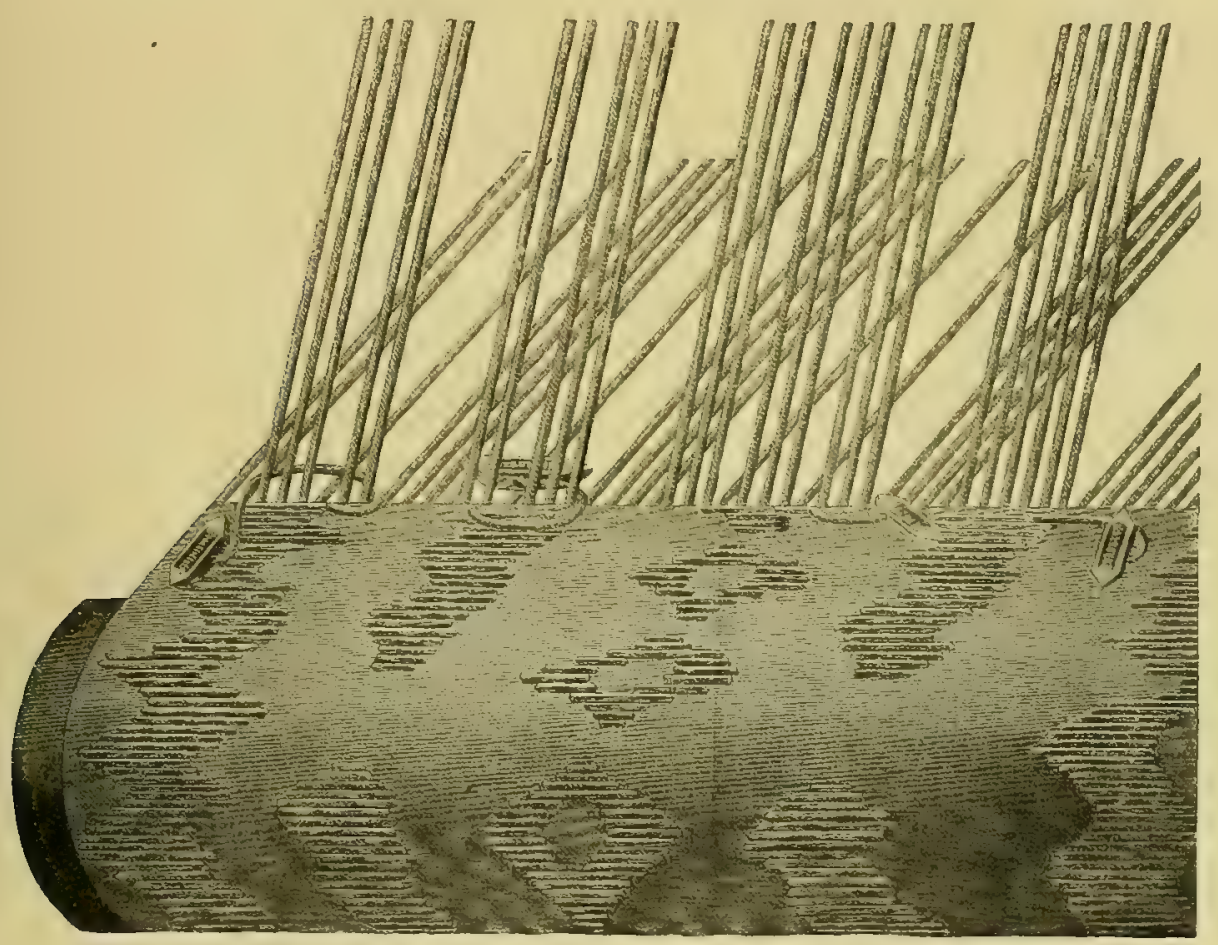

Schéma de la fabrication D'UNE SOIERIE bRochée (d'après l'Encyclopédie.)

répandue fait considérer un peu toutes les différentes soieries à dessins comme des étoffes brochées, à tel point que la dénomination est devenue courante, mais il y a là cependant une erreur sensible; le broché est un procédé de tissage bien défini et qui n'est pas d'un emploi aussi général qu'on pourrait le croire, s'il fut d'ailleurs connu, d'après maintes affirmations, de toute antiquité.

Dans le lancé, comme nous l'avons vu, on peut, par la manueuvre de navettes supplémentaires, faire passer une ou plusieurs trames en accompagnement ou en surnombre de la trame de fond pour contribuer à la décoration du tissu; ces trames, bien entendu, passent d'une lisière jusquà à l'autre, ou tout au travers, en langage de tisseur. Or, brocher c'est aussi ajouter au tissu une ou plusieurs trames supplémentaires, mais ceci seulement 
à des places voulues et choisies de l'étofte, un peu comme l'on exécute un ouvrage de broderie avec l'aiguille.

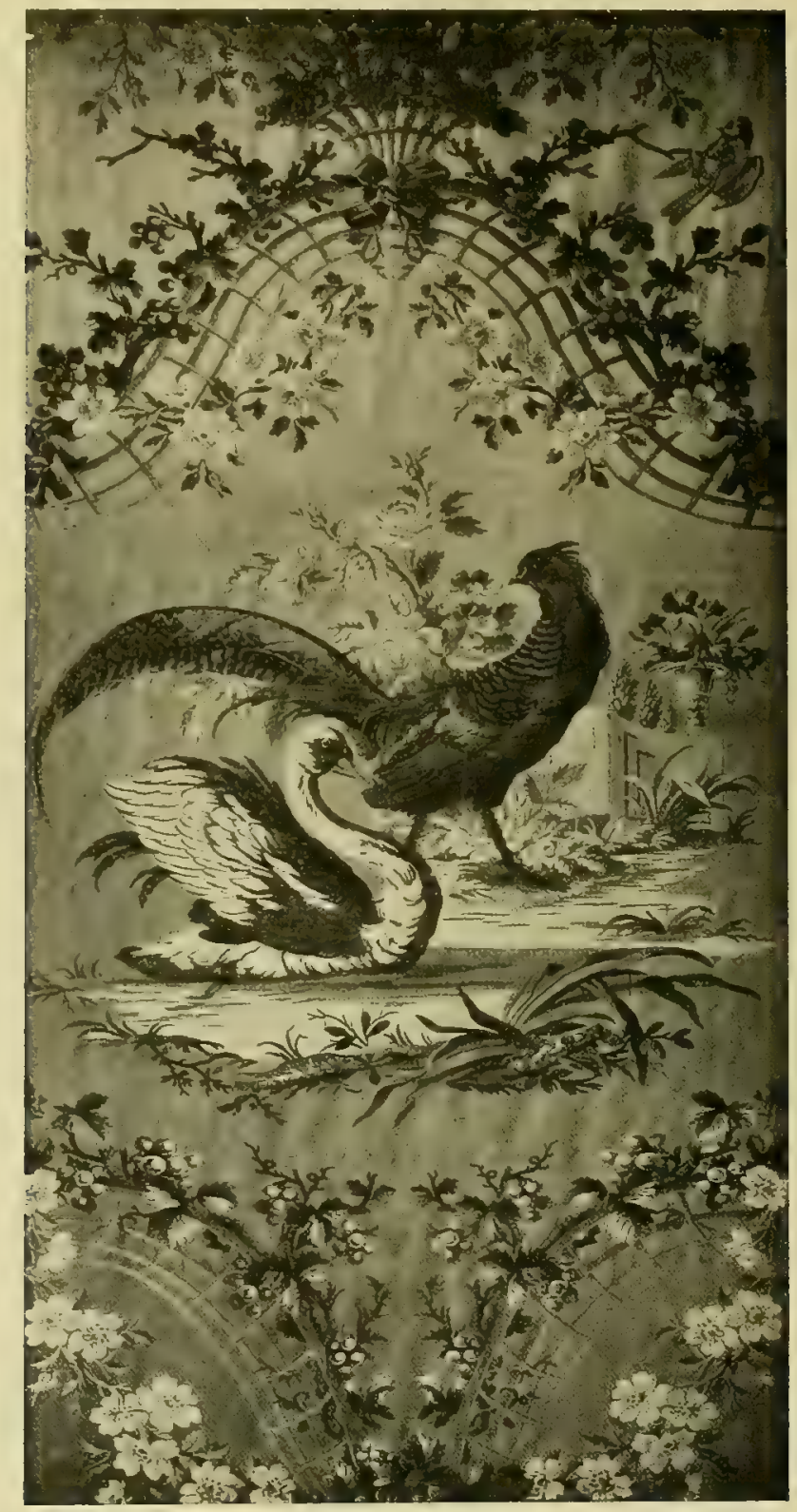

SOIERIE BROCHÉE, DÉCOR DIT " AU FAISAN 》.

(Composition de $\mathrm{Ph}$. de Lassalle.)

On forme de la sorte sur le fond, qui a sa trame propre et se tisse pour ainsi dire indépendamment, des motifs, fleurs, ornements, etc., plus ou 
moins détachés les uns des autres, ou brochés semés, et parfois au contraire sans séparation ou interruption en ce cas: brochés suivis, tout ceci étant déterminé, réglé par l'ordonnance du dessin.

Le travail s'exécute au moyen de petites navettes spéciales appelées espolins (d'où le terme espouliner parfois employé pour brocher) que l'on passe sous ${ }^{1}$ les fils de la chaîne, ceux du moins que l'on a fait lever en conséquence par prises convenables et, soit à la main, successivement dans toute la largeur de l'étoffe, d'après le système archaïque encore usité et imposé même quelquefois, soit avec le secours d'un appareil, le battant brocheur que manouvre le tisseur et qui effectue simultanément tous ces passages avec une simplification très évidente de main-d'œuvre.

On reconnaît très bien un tissu broché en le regardant à son envers; on voit que les trames brochées ne sont pas continues dans la largeur totale, mais limitées à des places occupées par le dessin; elles se trouvent disposées fil à fil ou coup par coup, toujours parallèlement les unes aux autres, à l'inverse de ce qui se passe dans la broderie où le fil entraîné

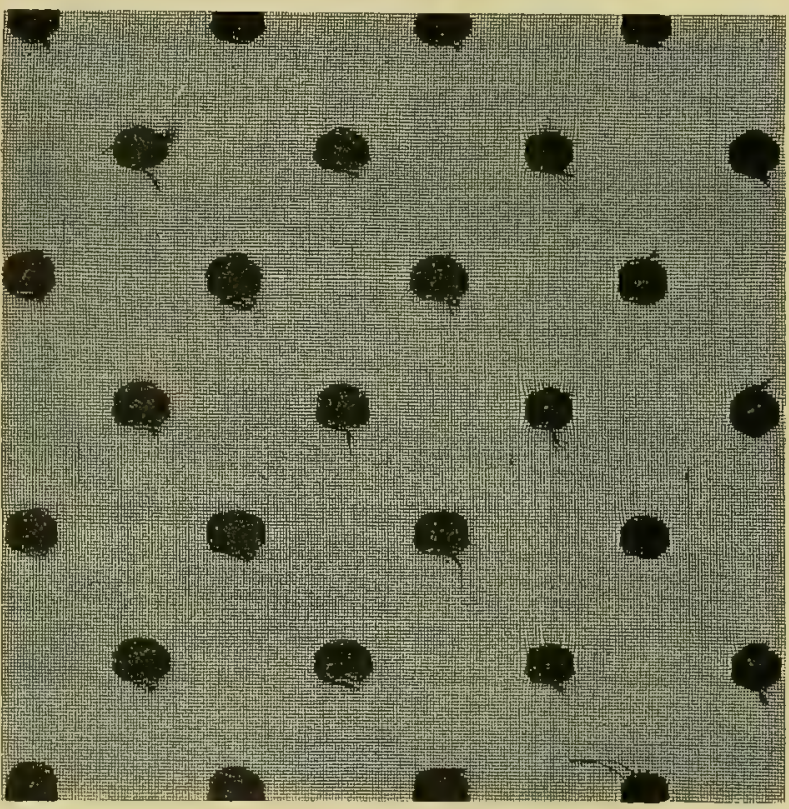

Grenadine brochée. par l'aiguille se meut en tous sens, et aussi bien à l'endroit où le dessin est figuré qu'à l'envers, dans les flottés ou brides qui se forment obligatoirement d'un motif à l'autre.

L'avantage du broché sur le lancé est de réaliser d'abord l'économie résultant de 1'emploi de la trame de soie juste comme et où il le faut pour les besoins de la décoration, car souvent une trame lancée ne figure à l'endroit du tissu qu'en des points restreints de sa largeur et, pour le reste, doit être dissimulée, liée à l'envers, ou même découpée et enlevée après le tissage, et par conséquent la soie est perdue en grande partie, ce qui narrive pas pour la trame brochée; il est beaucoup plus aisé de multiplier le nombre des trames brochées, et, par suite, des couleurs exprimées sur le tissu, que par tout autre procédé de tissage.

Puis, avec la mème utilité économique, le broché permet d'employer avec plus de facilité, surtout pour produire un dessin bien en relief et très varié d'aspect, des trames particulières, comme la chenille, l'or, l'argent, la

1 Il ne faut pas oublier que la plupart du temps les soieries se tissent : endroit dessous. 
cannetille, etc., toutes matières dont la grosseur et la nature sont des obstacles assez sérieux pour le tissage à la navette ordinaire.

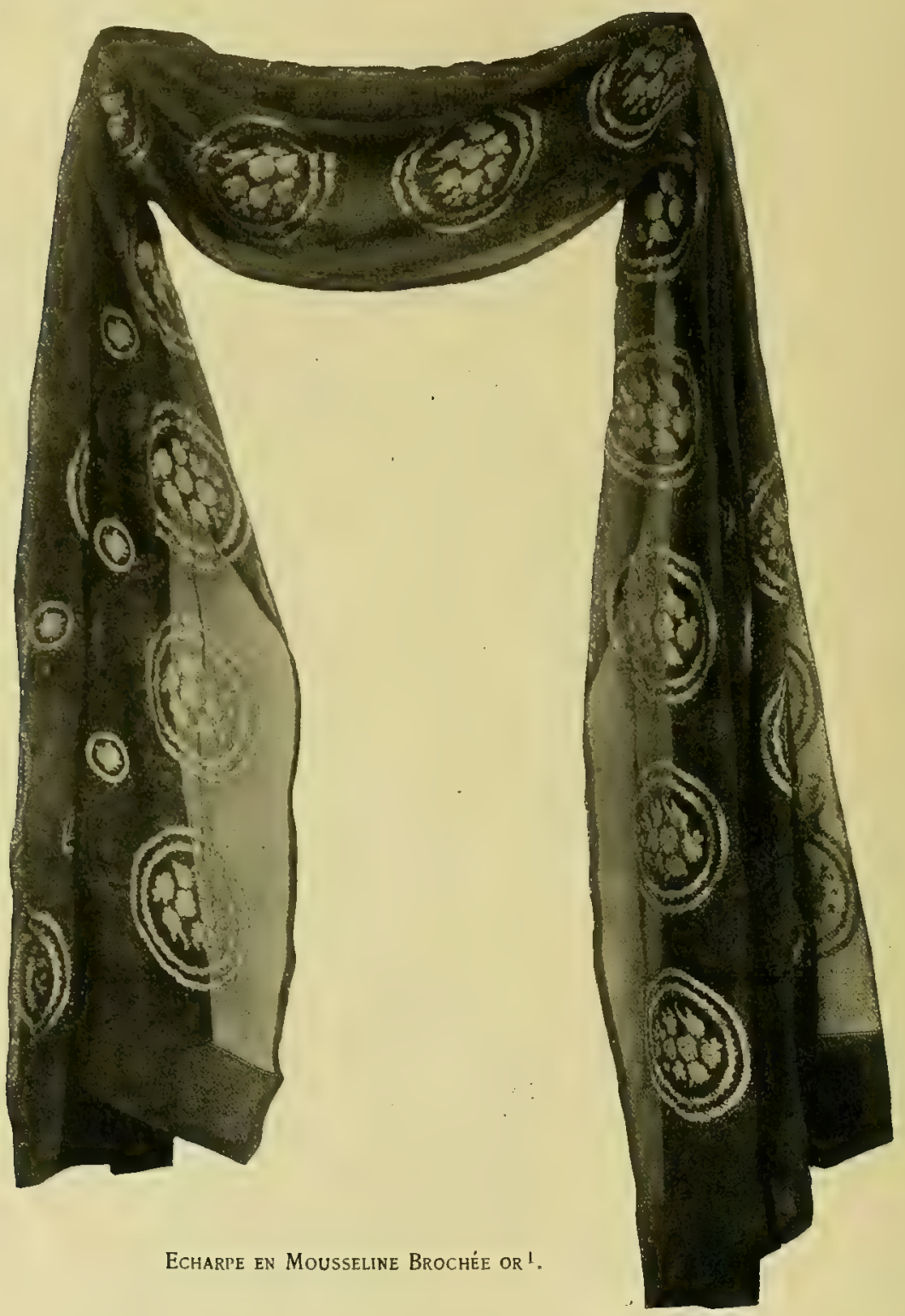

Il est enfin possible de combiner le lancé avec le broché et celui-ci encore avec le damassé, ce qui augmente les ressources de traduction plus exacte du dessin avec tout le détail et aussi toute la richesse que le compositeur lui a donnés sur son esquisse.

1 Communiqué par M. H. Bertrand, à Lyon. 
Aussi la variété des soieries brochées est-elle fort grande; elle comprend de belles étoffes d'ameublement ou d'ornement d'église dont la décoration largement entendue comporte souvent des parties exécutées en rehauts d'or et d'argent, comme de plus simples étoffes pour robes, pour cravates, et s'étend à des variantes nombreuses et à des applications spéciales : par exemple, pour des bordures, coins ou sujets isolés, façonnés ainsi à des places désignées, comme dans le cas de certaines écharpes, ou bien encore pour orner de dessins les fonds légers offerts par les mousselines, les grenadines, crêpes, crêpons, tous tissus trop peu résistants et composés de trop peu de fils, trame et chaine, pour que l'on puisse utiliser ceux-ci pour l'office de la décoration; au contraire, par une trame brochée, insérée dans le tissu avec l'aide d'un battant brodeur ou brodeuse, habilement manœuvré, on arrive à exécuter sur ces tissus des pois, des fleurettes semées ou des dessins suivis ou courants dans un genre qu'on nomme fréquemment plumetis; ces dessins s'enlèvent en parties plus serrées, plus épaisses et généralement brillantes sur des fonds qui sont clairs et transparents.

Mention doit être faite encore du système de broché dit crocheté, c'est-à-dire dans lequel les différentes trames brochées viennent s'entremêler, s'entrelacer l'une à l'autre dans le tissu d'une façon plus subtile et ingénieuse à constater que facile à relater. Mais ce procédé, très habilement employé par les artisans de l'Inde, en particulier pour les célèbres châles de cette provenance et dont l'usage s'est restreint aujourd'hui à peu près unique- ment aux rubans, n'est à la vérité qu'un épisode spécial de cet art du tissage $\mathrm{du}$ broché dont on relève l'application sur un si grand nombre de soies anciennes, depuis celles fabriquées dans les ateliers byzantins, palermitains, etc., jusqu'aux productions inégalées de Philippe de Lassalle, qui tira, pour son compte, un parti très important et très fréquent de cette technique. 


\title{
SOIERIES FAÇONNÉES PAR LA CHAINE
}

\author{
POMPADOUR - DROGUETS - DOUBLE-CHAINE
}

Si dans le façonnage des soieries à dessins, la trame ou les trames, lancées, brochées, lisérées, etc., jouent le rôle important que l'on a vu, il n'en

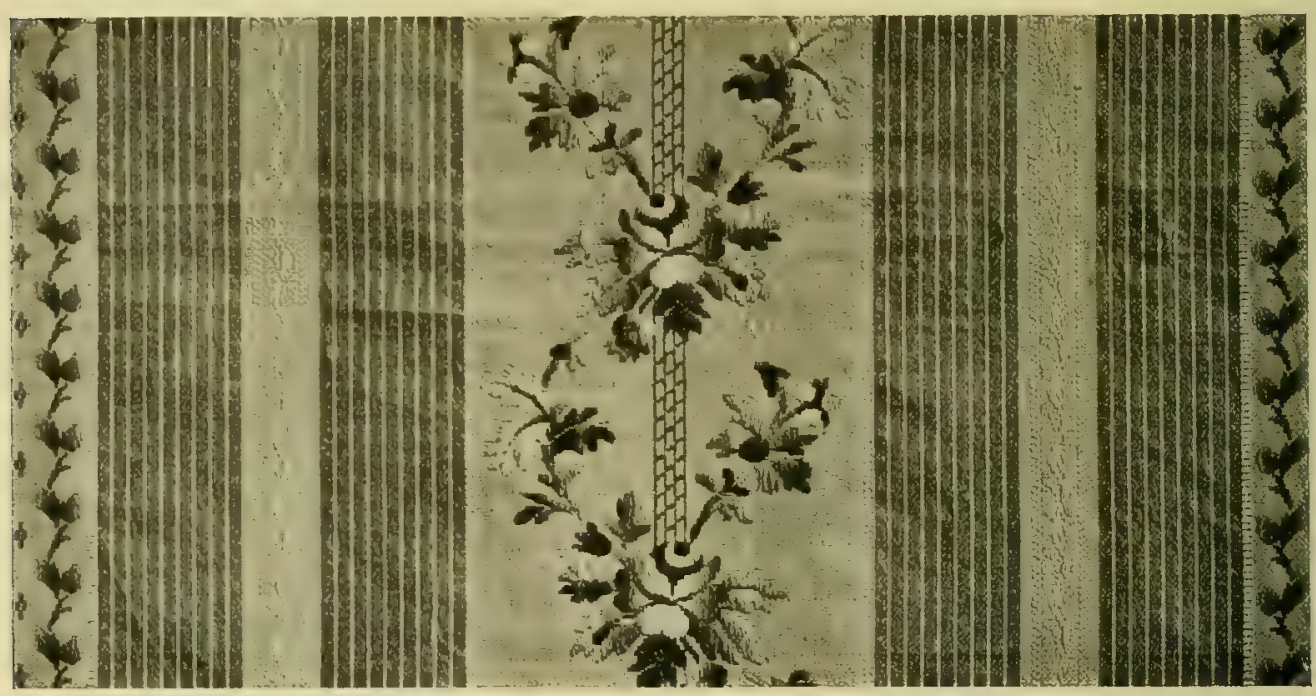

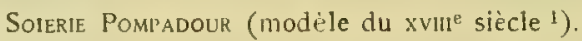

est pas moins vrai que l'on peut très bien, à des fins semblables, recourir au jeu des fils de la chaîne disposée en conséquence; on leur fait exécuter alors certains effets ou flottés, sortes de points dont l'importance est calculée et pour la bonne fabrication de l'étoffe et pour le meilleur rendement du dessin.

Ainsi par exemple, sur un fond de taffetas, même assez léger, ces flottés de la chaîne donnent par leurs points brillants et en relief le genre de soieries façonnées simples qui furent un temps en faveur sous le nom de Brillantines; elles ne comportent du reste, pour des raisons de solidité, qu'une ornementation fort réduite: pointillés, semés de pois, de fleurettes, de motifs, les uns et les autres forcément menus de proportions et pouvant au reste servir de fond à d'autres parties plus importantes du dessin, faites celles-ci par du broché ou autrement.

Mais les manières plus subtiles et compliquées de décorer un tissu de

1 Communiqué par MM. Tassinari et Chatel. 
soie par le moyen des fils de chaine sont nombreuses et offrent maintes ressources variées; nous n'en retiendrons que quelques-unes plus typiques.

Que l'on prépare en effet ces fils à l'ourdissage en les disposant fil à fil par, supposons: un blanc puis un bleu, et voici le moyen de faire un taffetas ou un gros grain dont les côtes transversales seront alternativement bleues et blanches ou à deux pas; cet arrangement très pratiqué en tissu uni est connu surtout sous le nom de Haïtienne et l'on en trouve parmi les soies anciennes de fréquentes applications; mais de plus, en adoptant ce dispositif pour un métier de façonné, on pourra obtenir sur ce fond des flottés, uniquement par les fils bleus, ou par les blancs, ce qui permet une interprétation du dessin d'après ces variations.

Autre combinaison de façonné toujours par la chaine: elle consiste à ajouter dans certaines parties de l'étoffe et, de préférence, sur un fond de taffetas pur, une ou plusieurs chaînes spéciales de supplément; on les compose de soies de diverses couleurs, fréquemment ombrées et choisies pour que les flottés de ces chaînes figurent des fleurs accompagnées de feuillages ou d'ornements légers, le tout se disposant sur le fond presque constamment blanc, en lignes forcément droites formant rayures. C'est le genre de soieries que l'on a appelé et que l'on appelle encore : Pompadour, du nom de la célèbre marquise, qui a été attaché à tant de choses artistiques diverses, produites de son temps. Et, de même qu'il y eut un style Pompadour dans l'ameublement, il y eut des costumes Pompadour ou des robes à la Pompadour; elles étaient faites précisément avec les soieries dont nous parlons et dont la mode, inaugurée sous Louis XV, se continua jusque sous le règne de Louis XVI avec la faveur la plus soutenue, et ceci explique la variété véritablement prodigieuse qui fut créée de ces charmantes étoffes aux colorations fraîches et au décor si justement proportionné, le plus souvent agrémenté de bandes en accompagnement du façonné, faites de cannelé, de satin, de sergé, chevron ou autres ingénieuses et agréables utilisations. On s'est borné depuis à reproduire, textuellement pour la plupart, ces dispositions prises comme modèles de goût et d'élégance.

Les mêmes étoffes ou analogues, destinées aux usages de l'ameublement sont aussi connues sous le nom un peu désuet dé Mexicaines, tandis qu'un terme technique très exactement expressif les classe comme des poils tratnants; poil voulant désigner ici les chaînes supplémentaires, traînant ou flottant à l'envers du tissu quand elles ne figurent pas le dessin à l'endroit.

Autre soierie façonnée qui emprunte aux fils de chaîne la majeure partie, le fond caractéristique de sa décoration: le Droguet de soie, qu'il faut se garder de confondre avec les étoffes communes du même nom que l'on fit autrefois en laine, ou en fil et laine mélangés pour certaines sortes de tapis ou des garnitures très ordinaires de lits; les droguets de soie, au contraire, s'adressent à des emplois plus relevés.

Ils sont formés d'une sorte de fond dissimulé qui est du taffetas, sur lequel les fils de même couleur d'une chaîne spéciale tracent à leur tour comme un fond armuré, par des flottés liés assez régulièrement en gros sergés, chevrons, etc., et en même temps les lignes du dessin qui est le plus souvent achevé par des effets d'une autre coloration fournis par des trames lancées et liées dans le fond; les dessins de droguets sont de petite proportion, souvent à bouquets 
très légers enfermés dans des sortes de compartiments, dessinés par de fins contours de trames. Il est difficile de donner de ces droguets une idée très exacte et très marquée tellement la composition en fut variée, car la fabrication de ces étoffes si recherchées jadis constituait à Lyon dès le Xrir siecle une des principales branches du commerce de cette ville.

$S$ 'il faut en croire Paulet, dessinateur réputé de Nîmes, auteur compétent d'un très important ouvrage qui fit longtemps autorité: l"Art duFabriquant d'Etoffes de Soies, publié de 1773 à 1776 , cette supériorité lyonnaise

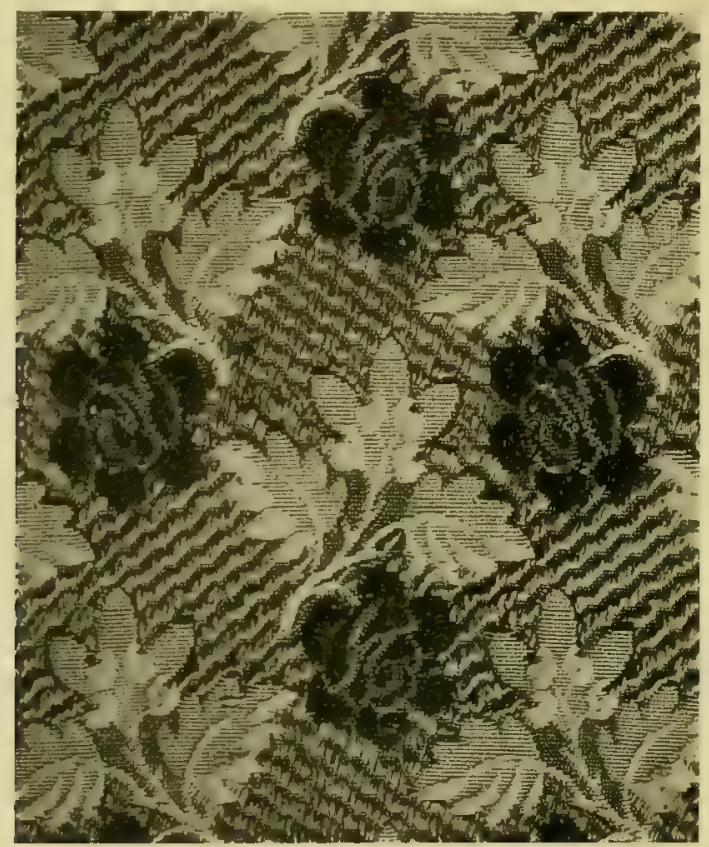

Droguet !

manufacturière pour les droguets était tellement incontestable qu'il dit quelque part : "Je suis certain que Nismes, Tours et Paris n'en ont pas fait travailler vingt métiers tandis que Lyon en avait près de deux mille »; et plus loin : "Il y a vingt-cinq ans, on regardait encore comme très habile l'homme qui était capable de monter un métier à faire des droguets. „ Et il en fait remonter l'invention à Galantier, célèbre spécialiste avignonnais de la soie, parmi de nombreux artistes de cette époque.

On ne disposait pas alors des moyens qui ont facilité beaucoup ce genre de fabrication; c'est avec les métiers à la marche ou encore à la petite tire et en s'aidant d'arrangements très particuliers, pour lesquels les gens de métier comme Paulet étaient passés maitres de par leur expérience, que l'on

1 Communiqué par MM. Tassinari et Chatel: 
tissait ces soieries compliquées où chacun mettait son habileté et son ingéniosité, avec un certain tour de main gardé secret le plus possible: Egyptiennes, Prussiennes, Ambroisiennes, Musulmanes et Droguets de toutes sortes: Maubois, à la Reine, miniatures, lisérés, lamés, ceux-ci rehaussés par l'emploi de l'or et de l'argent. Il y eut des droguets pour tous les goûts, aussi bien pour les robes simples que pour celles de cour et en grand nombre aussi pour les vêtements d'hommes, habits, gilets de cette époque élégante.

On peut leur assimiler la Dauphine, très riche étoffe de principe analogue, enrichie de nombreuses trames de couleurs diverses et que l'on présume dater de l'époque où l'archiduchesse Marie-Antoinette, épouse du Dauphin futur Louis XVI, parut à la cour de Louis XV vers $177^{\circ}$; c'est à Lyon que cette étoffe fut exclusivement fabriquée.

Sous l'appellation Double-chaîne, on classe d'habitude aujourd'hui des tissus de soie façonnés, entendus comme ces genres à fonds satinés sur lesquels des dessins apparaissent formés d'un satin d'une autre couleur qu'on ne s'explique pas au premier abord; c'est la deuxième ou la double chaîne, dissimulée dans le fond sous la première, qui permet d'obtenir cet effet fort curieux et dont on a souvent tiré un parti très intéressant, pour des soieries presque toujours d'un certain prix. 


\section{GAZES. CRÊPES ET VELOURS FAÇONNÉS}

Décorer, façonner les gazes de soie, tissus fort légers, où par définition les fils sont en petit nombre et peu serrés, semble un problème assez délicat.

Il se résout cependant élégamment et de plusieurs manières.

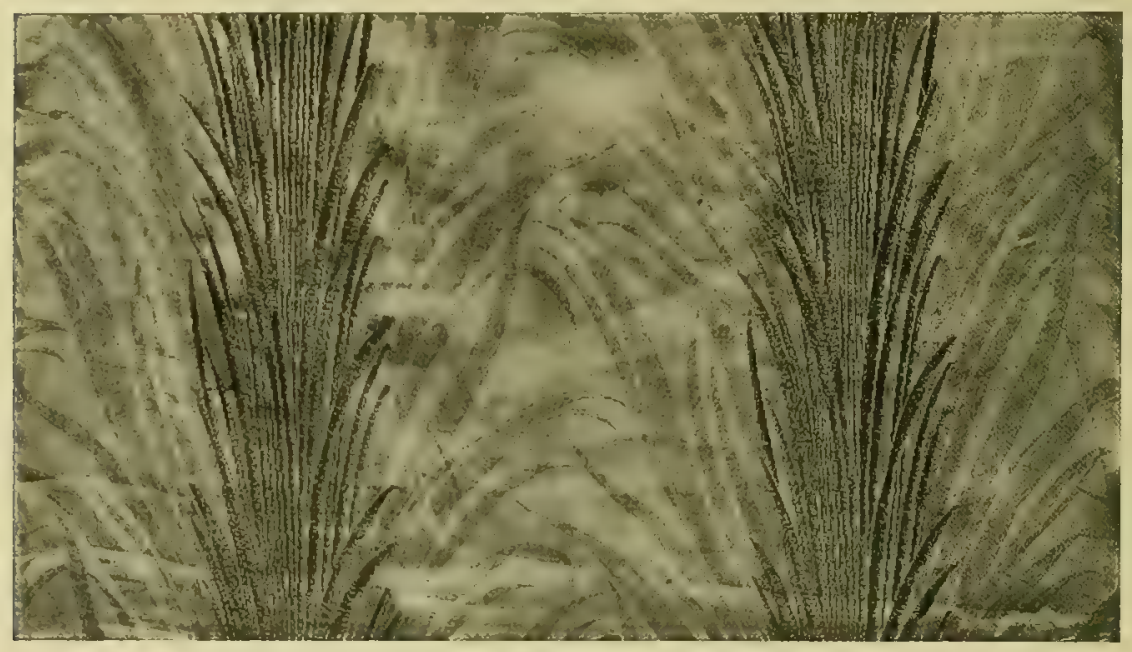

CRÊPe de Chine façonné et broché or ${ }^{1}$.

On peut d'abord, comme nous l'avons déjà noté, appliquer le système du broché tant sur la gaze proprement dite ou à fil de tour que sur les mousselines, voiles, etc., et varier le genre par les différentes sortes de trames de broché employées, brillantes ou mates, fines ou grosses, à une ou plusieurs couleurs, etc.

Recourir aux fils clairsemés de la chaine d'une gaze pour former des flottés et, par suite, des dessins, paraît une tentative hasardeuse et cependant qui donne un résultat très exploité commercialement; toute la catégorie des gazes façonnées, gazes diaphanes et autres, en est la preuve. Il est vrai que, souvent, le secours de la trame vient faciliter les choses, trame qui peut être de couleur opposée à la chaîne, et animer la coloration.

Et puis, si l'on augmente le nombre des fils dans toute la largeur du tissu ou bien dans certaines parties, on arrive à faire du satin, satin qui joue en opposition avec la gaze et dont on découpera parfois les fils quand,

1 Communiqué par MM. Coudurier, Fructus et Descher. 
devenus inutiles par leur passage à l'envers, après avoir fait leur effet à l'endroit, ils pourraient enlever de la transparence du fond.

Enfin, que 1'on veuille, sur un fond de gaze, faire se détacher des motifs formés de l'étoffe de soie de la contexture la plus opposée, c'est-à-dire le velours, il suffit que l'on ne soit pas arrêté par des considérations de soins particuliers de temps ni dargent pour réaliser la chose-sans difficultés insurmontables. Si l'on pousse la recherche plus loin, en employant le métal pour avoir les gazes façonnées d'or et d'argent, les perles pour les gazes perlées, etc., on atteint dans ce compartiment de la soie aux genres les plus riches.

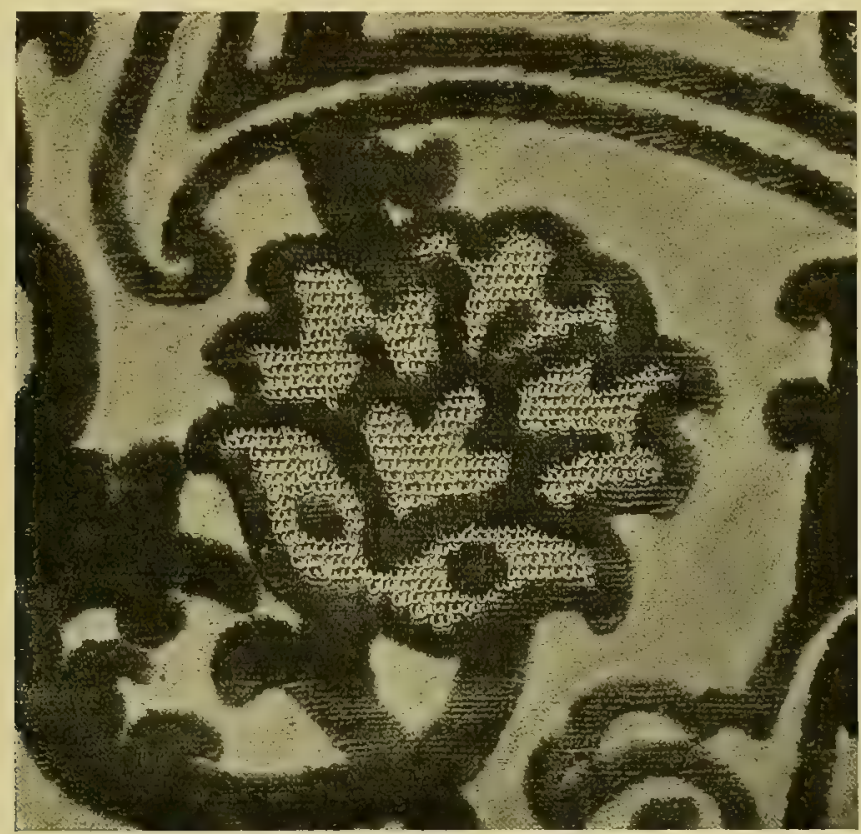

VELOURS AVEC BOUCLÉS D'OR 1 .

Comme les gazes, les crêpes ou, plus précisément, les crêpes de Chine peuvent être brochés ou façonnés par les effets de chaîne et de satin et, de plus, par des trames lancées.

Les crêpes de Chine à dessins ont été comme le début des soieries damassées et brochées, tout à fait souples, fabriquées d'après le mode du teint en pièces, obligatoire on le sait dans le cas du crêpe, et devenu de pratique courante grâce aux progrès actuellement faits dans cette voie; si bien que l'on traite parfaitement des étoffes mêlées de fils d'or, sans que ceux-ci soient altérés définitivement par les opérations de la teinture et qu'on peut leur redonner ensuite à nouveau leur éclat primitif.

Pour quant au velours, la complexité et la délicatesse de ce tissu, dont

1 Communiqué par MM. Tassinari et Chatel. 
nous croyons avoir donné quelque idée au chapitre de l’ « uni », ne semblent pas avoir été longtemps un obstacle à l'idée de sa décoration par le tissage même.

Il y a d'admirables velours persans de toutes époques qui unissent à la

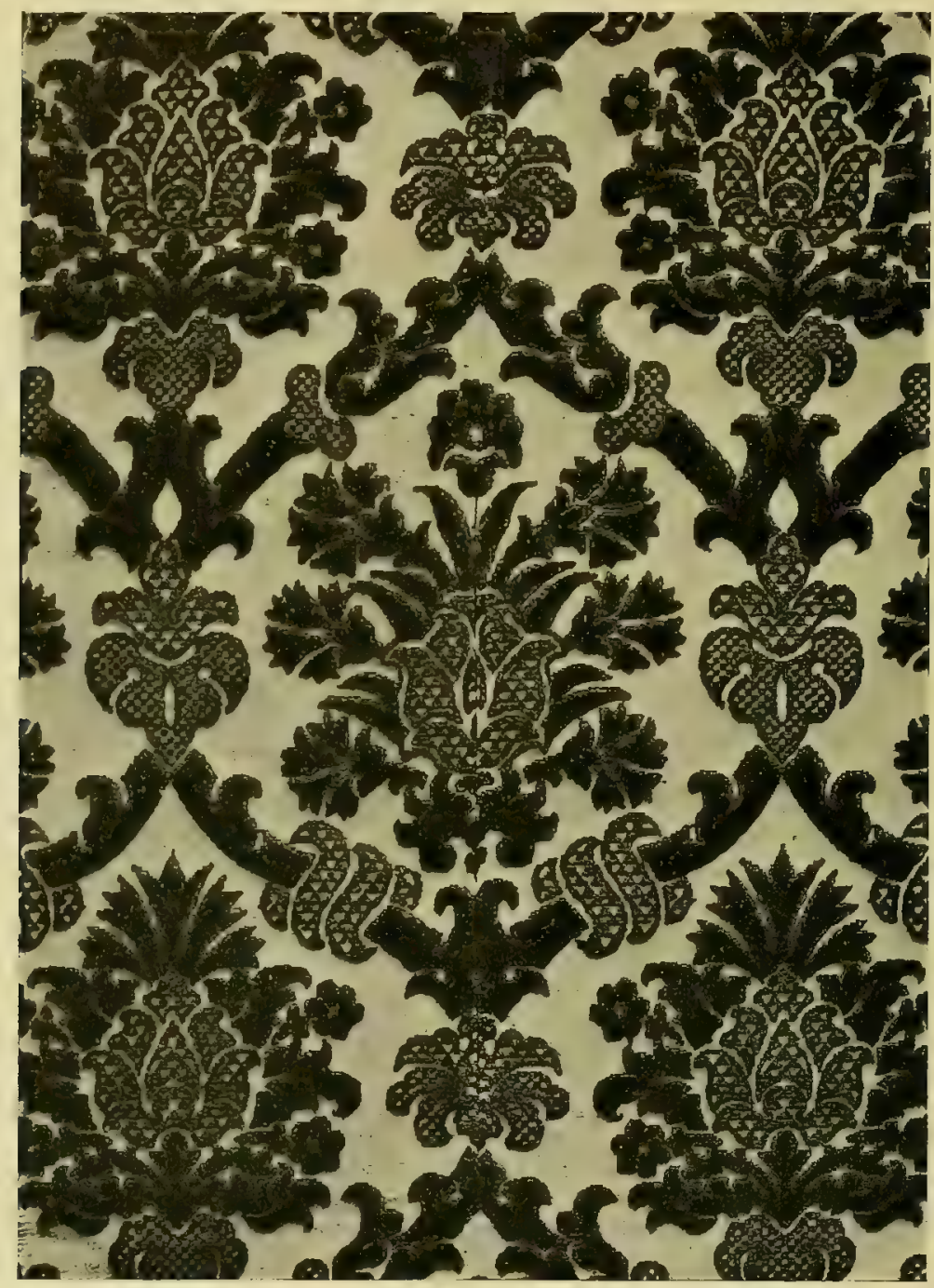

Velours de Gènes.

technique la plus rigoureuse et dont les méthodes sont encore appliquées la coloration la mieux comprise, et la plus artistement nuancée par le mélange, dans un même velours coupé à surface unie, de fils de teintes opposées; velours dits aujourd'hui à plusieurs corps, deux corps, trois corps, etc., ce qui entraine pratiquement de grosses complications de tissage, en nécessitant l'emploi de plusieurs cantres ou ensembles de nombreuses petites bobines 
chargées de soie; à cause de l’inégale répartition du velours sur une étoffe façonnée qui en comporte, on ne peut, en effet, placer les fils de soie destinés à former ces effets de velours sur un rouleau unique. Il faut laisser à chacun leur indépendance, ce que permet la séparation des bobines de la cantre.

Il y a d'autres velours orientaux anciens, présentant des dessins

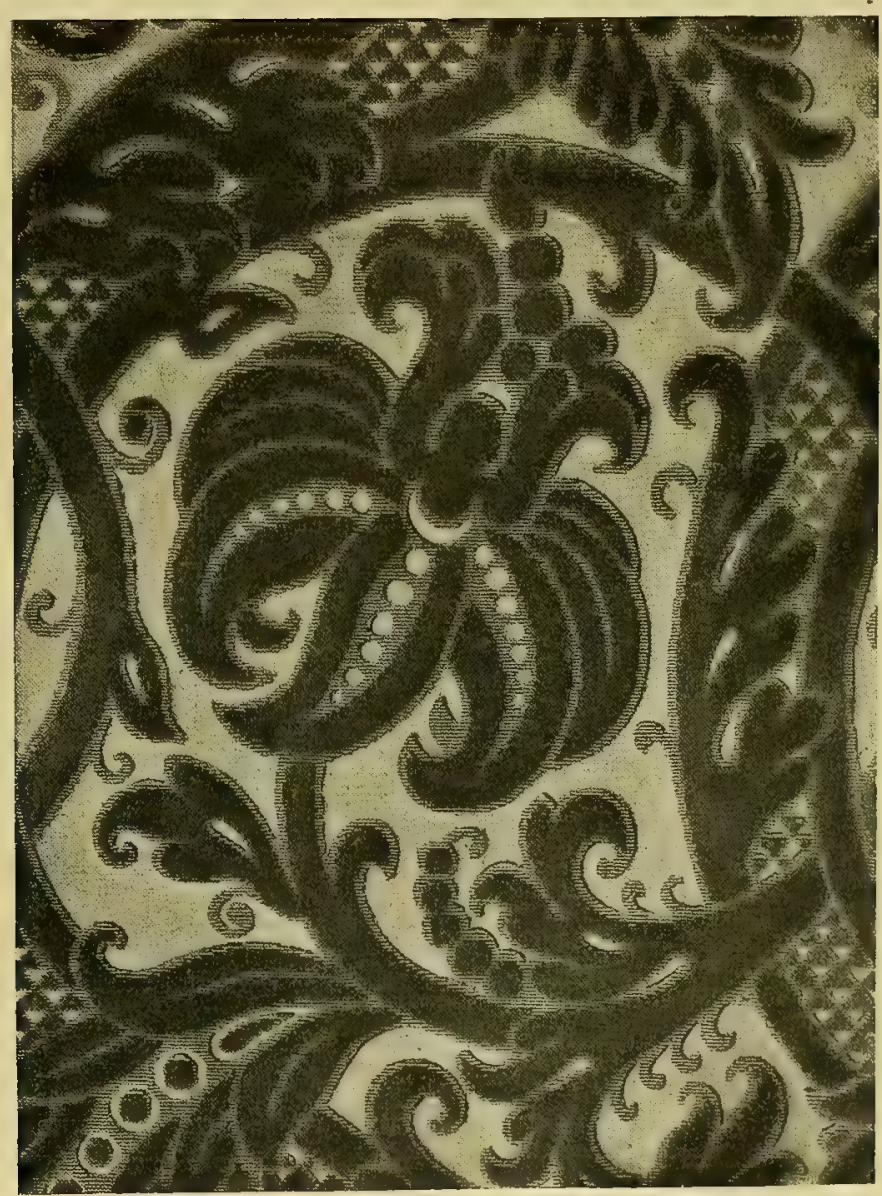

VELOURS CISELÉ.

dont la grandeur et la richesse étonnent autant que charme leur coloris et qui démontrent l'emploi fort habile dans leur fabrication de procédés très délicats: velours à deux hauteurs, avec deux poils coupés, l'un sensiblement plus haut que l'autre, obtenus par des fers appropriés - velours reposant sur un fond où apparaissent des trames de soie, ou de métal précieux - velours rehaussés de bouclés d'or par une technique si spéciale que la pratique s'en est perdue presque complètement, consistant à former sur le velours des bouclettes régulières d'un fil d'or ou d'argent qui figurent un dessin sur le dessin lui-mème. 
Après la magnificence des velours à l'époque médiévale, cent fois célébrée dans les poèmes, chansons de gestes et chroniques, surtout ceux ornés de broderies dont nous ne nous occuperons pas ici, la Renaissance italienne a fourni cette incomparable série si multipliée de velours façonnés, de style si compact, qu'ils restent comme des modèles avec leurs colorations chaudes, souvent cependant monochromes, soit qu'ils portent des dessins

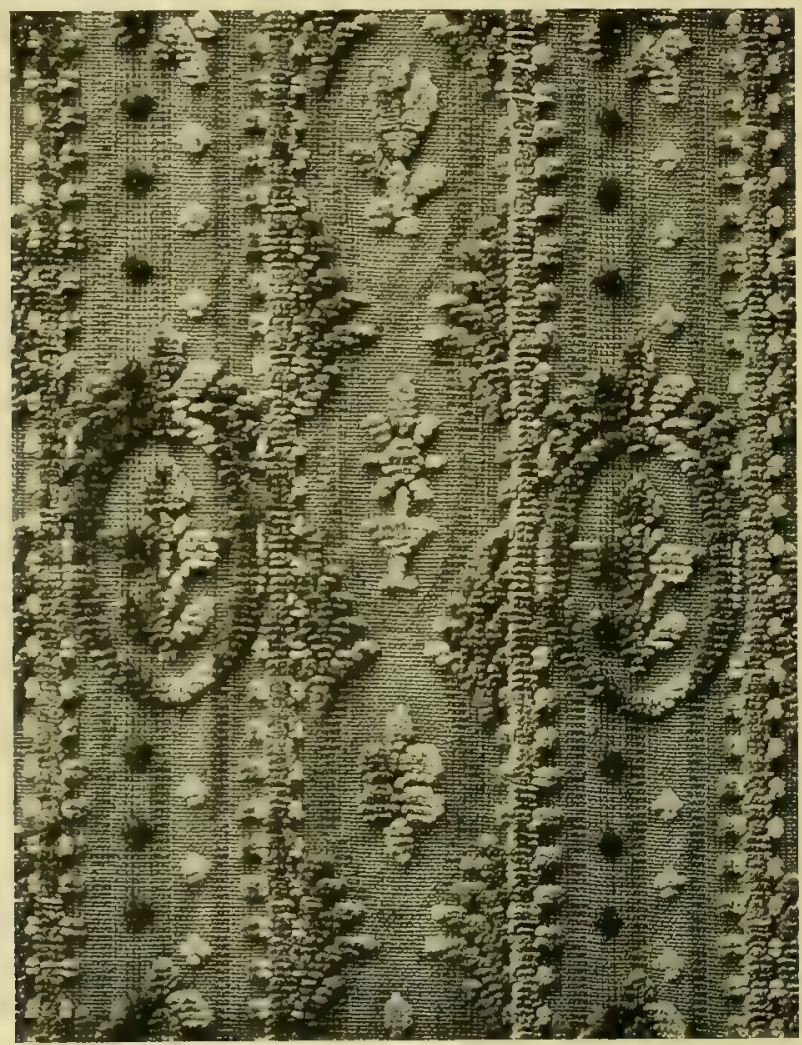

Velours miniature (modèle du xvill ${ }^{c}$ siècle ${ }^{1}$ ).

bien composés pour paraitre riches, dans leurs petites proportions, soit qu'ils atteignent au large développement des velours à grands ramages et, cette fois, polychromes.

Alors, Gènes triomphe dans la fabrication des genres de velours qui ont conservé toujours ce patronage réputé: velours à fond de satin, quelquefois à fond lamé d'or, sur lequel le dessin s'enlève en velours coupé, mélangé avec du velours frisé qui sert de préférence à faire les entourages, les sertis. C'est l'entente actuelle toute pareille du velours ciselé, les parties

1 Communiqué par MM. Tassinari et Chatel. 
en velours coupé formant le relief, et le frisé par oppositions les parties basses du dessin, en imitant un réel travail de ciselure. Et il faut bien se garder de confondre ces relours ciselés par tissage avec ceux dits frappés comme on le fait assez souvent à tort; ces velours frappés sont le plus généralement de laine, et subissent l'action de plaques ou rouleaux métalliques gravés en relief, chauffés et pressés sur l'étoffe, qui marquent

ainsi des dessins formés par le poil qui reste couché à ces places.

Et l'histoire glorieuse du velours façonné se continue pour l'ameublement avec les magnifiques velours à parterre, à jardins, ou jardinières du règne de Louis XIV, traités à la «façon de Gênes », avec la complication de corps (ou cantres) multiples; les dessins en sont fort grands, entendus le plus généralement à retour, c'est-à-dire, comme nous l'avons vu, compris pour que la partie de droite occupant la moitié de la largeur de l'étoffe soit exactement la même que celle de gauche mais retournée, c'est-à-dire de sens inverse ; ceci combiné pour que l'ensemble ne soit pas coupé au milieu, de façon disgracieuse, et donne, au contraire, une belle impression de richesse décorative et de grandeur. Bérain, le décorateur célèbre, ne dédaignait pas alors, nous l'avons dit, de tracer l'esquisse du splendide et somptueux velours, enrichi d'or, qui fut tissé dans la manufacture de Marcelin Charlier, à Saint-Maur, pour lechâteau de Versailles.

D'autre part, pendant tout le XVII" et le $\mathrm{XVIII}^{\mathrm{e}}$ siècle, la richesse des vêtements pour les hommes de cour, exclusivement ou presque faits de soie et de velours, encourageait quantité d'artisans et de dessinateurs (parmi lesquels on a cité souvent,

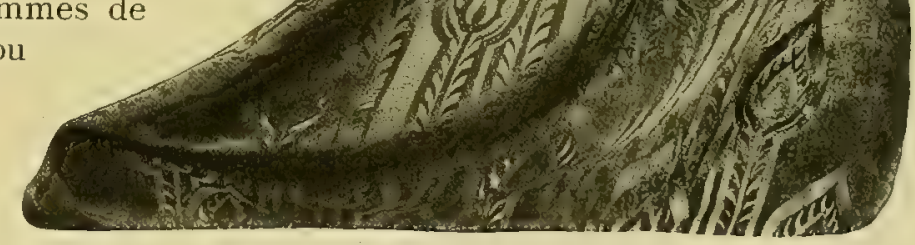
comme l'un des plus

Velours faÇONNÉ, TRAMÉ OR, TEINT EN rIÈCES L. habiles, Dacier de Lyon) à varier par d'incroyables recherches d'exécution le façonnage des velours.

La perfection fut atteinte peut-être sous Louis XVI, avec un maximum de finesse d'effets, et d'élégance parfaite. Il y eut, comme nous le relevons sur un tarif accompagnant une carte d'échantillons de fabricants qui date d'avril I 780, des velours «mignatures » 3 corps à 35 livres l'aune, comme des velours à 《points d'Angleterre 》 à 28 livres, des velours « frisés nués 》 á 18 livres, des velours à «bordures fond couleur 》 ̀̀ 52 livres et bien d'autres comme des velours pour poches d'habits, à fond d'or, etc., etc. Il semble que

1 Communiqué par MM. Coudurier, Fructus et Descher. 
l'imagination de leurs créateurs ait été inépuisable et il nous souvient d'avoir vu sur d'anciens livres de références de cette époque près de douze cents échantillons de ces velours, tous différents.

Sous le Premier Empire, avec de très beaux velours façonnés d'ameublement, puis sous 1a Restauration et, plus tard, sous Louis-Philippe, avec des genres de velours pour vêtements d'hommes réduits à l'emploi du gilet, on a épuisé peut-être, en les rééditant sous d'autres formes, sur différents fonds, et de diverses manières plus ou moins habiles, les multiples combinaisons déjà connues pour façonner ce genre d'étoffe.

Notre époque s'en est à peu près tenue là, et n'a rien produit de très particulièrement sensationnel : ce sont les procédés annexes du tissage, de la teinture en pièces, de l'apprêt et du finissage qui ont surtout apporté de l'inédit, dans une branche textile où il n'est pas facile d'innover tant elle a été exploitée avec art depuis longtemps.

L'exemple donné par la figure placée plus haut a été choisi parmi ces beaux velours mélangés d'or que l'on réussit aujourd'hui parfaitement d'une façon qui aurait semblé un tour de force irréalisable il n'y a pas très longtemps. On les tisse d'abord avec de la soie écrue, en teignant ensuite les pièces, avec la précaution de redresser soigneusement à l'apprêt le poil du velours malmené évidemment par la teinture, comme de réaviver l'éclat du métal terni dans le bain colorant; et l'on obtient de la sorte, avec une facilịté presque déconcertante, toute la beauté, la richesse des velours à l'ancien système pour ceux-ci qui possèdent, en outre, une souplesse des plus goûtées dans les emplois: robes et manteaux. 


\section{BROCARTS}

Avec les brocarts nous arrivons à la classe des soieries façonnées les plus riches, et par les proportions du dessin et surtout par le mélange constant qu'elles comportent de l'or et de l'argent avec la soie.

A maintes reprises déjà nous avons cité l'emploi combiné de fils faits de métaux précieux avec des fils de soie mais, dans le cas des brocarts, le rôle des premiers est prépondérant; la soie ne sert pour ainsi dire que de support et de lien à la matière rare quị joue avec tout son éclat dans le fond de l'étoffe comme dans les différentes parties du dessin.

Il en est ainsi du moins pour le brocart fabriqué comme ceux dont le type le plus exact est représenté de nos jours par les belles étoffes d'ornements d’églises; le nom « brocart » est devenu en effet et reste la plupart du temps générique et sert à désigner, sous une rubrique commode, quantités de soieries mêlées de métal, comme par exemple les damas, les gros de Tours, brochés d'or et d'argent, qui ne sont pas des brocarts à proprement parler. Les documents nous apprennent aussi que, des l'origine, les brocarts étaient des tissus tout d'or et d'argent, quelquefois des deux ensemble, soit en chaîne, soit en trame.

On peut dire que ces étoffes faisaient partie de la vaste famille des draps d'or, si goûtés au moyen âge, après que l'usage en eût été emprunté à l'Orient et à ses fastes et entre autres occasions au moment des Croisades. L'emploi de tissus dans lesquels on sut faire entrer l'or remonte aux époques les plus reculées, comme l'ont montré de savantes recherches. Une citation classique à ce sujet est celle du passage de l'Exode où Moïse dit que l'on coupa des lames dor qui furent réduites en feuilles très minces de manière à ce qu'on pût les tourner et plier pour les faire entrer dans le tissu des autres fils.

Pline, dans son Histoire naturelle, parle de l'art de mêler l'or à la laine pour en former un tissu précieux et en fait remonter l'invention au roi Attale, souverain asiatique.Lui-même avait vu, rapporte-t-il quelque part, Agrippine, femme de l'empereur Claude, assister près de son mari au spectacle d'une naumachie, couverte d'une tunique d'or pur tissé. Héliogabale, d'après Lampride, faisait usage d'étoffes d'or. Et les mentions d'habits d'or ou tissés de fils d'or ne sont pas rares dans les écrivains de l'antiquité; il est remarquable de constater que l'invention du fil d'argent paraît avoir été nettement postérieure à celle du fil d'or et les documents qui parlent d'étoffes faites avec de l'argent n'apparaissent que beaucoup plus tardivement.

Dès que la soie fut produite en assez grande abondance, ce fut une raison toute naturelle pour la substituer à la laine comme support des fils faits avec les . métaux précieux. Les historiens se sont livrés à de nombreuses investigations pour arriver à déterminer le moment précis de cette application et la composition exacte, par exemple, de l'habit offert par Charles le Chauve au pape 
Nicolas $I^{\mathrm{er}}$, ou des tentures tissées d'or et enrichies de perles dont Dagobert, assisté de son ministre, l'orfèvre saint Eloi, fit couvrir les murs et jusqu'aux colonnes de l'église Saint-Denis qu'il avait fait construire; mais l'incertitude est restée grande pour tout ceci, aussi bien que pour l'identification des étoffes du même genre, offertes par Justinien à l'église Saint-Pierre ou de celles qui formèrent les riches vêtements dautels dont plusieurs pontifes ou rois firent l'offrande à des basiliques.

S'agissait-il bien là du chrysoclavum ou auroclavum, noms que l'on a relevés dans les auteurs du temps? On en est réduit à des hypothèses, mais il est surprenant en tous cas de voir, en suivant le cours des âges, la quantité de tissus ornés d'or, qui furent en usage aux époques anciennes; ce sont : les araps à or battu ou à battus à or et encore draps de soie à or ouvrés, cités à chaque instant dans les chants des trouvères, les étoffes précieuses du

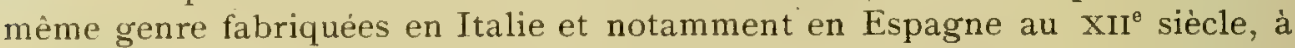
Alméria, ville célèbre pour sa fabrication, où il y eut jusqu'à mille métiers de brocarts, et les draps ct toilles d'or et d'argent qui furent employés à profusion pour la célèbre entrevue de François ${ }^{\text {er }}$ et de Henri VIII qui eut lieu entre Guisnes et Ardres en I 520 , au mois de juin, assemblée connue sous le nom de Camp du Drap dior; ce fut au point, dit un chroniqueur de façon pittoresque, que " plusieurs y portèrent leurs moullins, leurs forests, et leurs préz sur leurs épaules", et que les principales tentes, ajoute un autre, étaient " de drap d'or frisé dedans et dehors, tant chambres que galleries, et tout plein d'autres de drap d'or ras et toilles d'or et d'argent $\gg$.

L'Orient, où tous ces tissus magnifiques avaient pris naissance, gardait au mème moment toute sa réputation pour en produire que lion voit spécifiés dans les inventaires avec leurs provenances; Brantôme, faisant le portrait de Marguerite de Valois telle qu'elle lui apparut à Blois, à la procession, le jour de Pâques fleuries de l'an I57 I, s'exprime en ces termes : " Son beau corps, avec sa riche et haute taille, était vestu d'une robbe de drap d'or frisé. le plus beau et le plus riche qui fust jamais veu en France et c'était un présent qu'avait fait le Grand Seigneur à son départ de Constantinople à M. de Granchamp... vers lequel il était ambassadeur..., d'une pièce qui montait à quinze aunes, lequel Granchamp me dist qu'elle avait cousté cent écus l'aulne, car c'était un chef-d'œuvre. Luy même venu en France... la redonna à Madame, sœur du Roy, qui en fit faire une robbe, qui, pour la première fois, s'en para ce jour-là... et la porta tout le jour, bien qu'elle pesât extrêmement... " "

On connaît le passage bien souvent cité de la lettre de $M^{\text {me }}$ de Sévigné décrivant une robe portée par $M^{\text {me }}$ de Maintenon « dor sur or, rebrochée d'or, rebrodée d'or, et par-dessus un or frisé rebroché d'un or, mêlé à un certain or, qui fait la plus divine étoffe qui ait été jamais imaginée ».

Le brocart d'or était parmi les quatre draps, sur l'un desquels devaient exécuter leur chef-d'œuvre tous ceux qui aspiraient à être reçus marchands et maîtres-ouvriers en drap d'or, d'argent et de soie en la ville de Paris, aux termes du règlement corporatif de 1667 , car leur fabrication n'était pas localisée à Lyon et à Tours et la fabrique, à Saint-Maur, du sieur Charlier, fournisseur de Louis XIV, alimenta les réserves royales de nombreux et riches brocarts. 
On conçoit que des difficultés particulières se présentent pour le tissage des étoffes de soie dans la composition desquelles entrent des fils d'or et d'argent; et pour la préparation de ceux-ci, il est nécessaire non plus de « battre » l'or comme jadis, mais de l'étirer tout d'abord en une fine lame

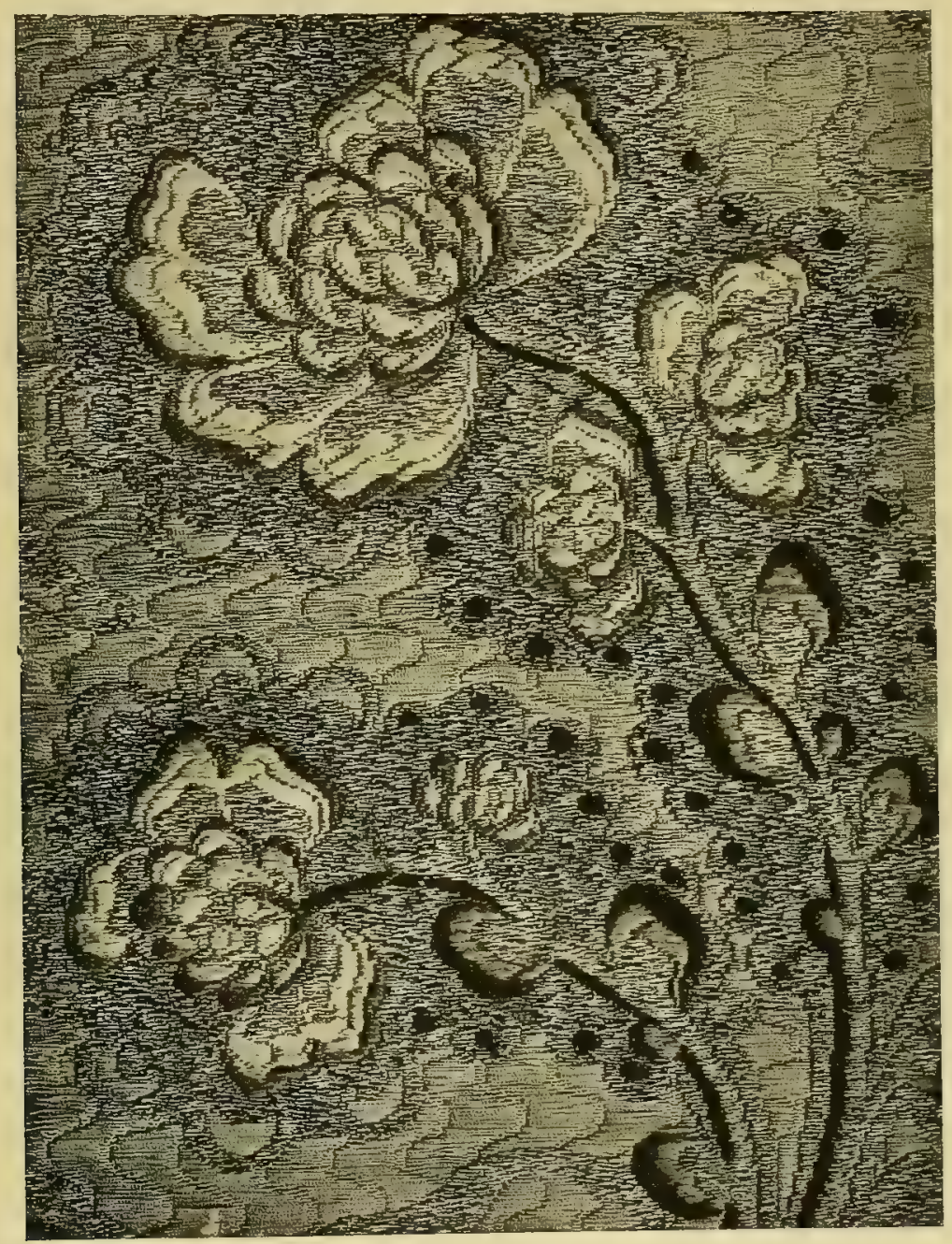

BROCART OR, ARGENT ET SOIE '.

très mince et flexible obtenue par le laminage spécial d'un fil déjả très fin et ductile obtenu par le passage du métal dans une filière, faite d'un diamant perforé; puis cette lame ou or trait ainsi obtenue, il s'agit de l'enrouler en spirale, sur une âme, ou fil de support, qui est ordinairement maintenant de

1 Communiqué par MM. Tassinari et Chatel. 
coton teinté en jaune, pour l'or, afin de ne pas trancher sur la teinte du métal, et parfois de soie; c'est le travail de 1 'ouvrier dit guimpier. Plus les spires sont serrées les unes contre les autres sur l'âme et plus le filé qui en résulte prend de prix. Anciennement, on s'est servi de supports, pour le métal, faits avec diverses substances comme par exemple la baudruche et la très fine cordelette de boyau; les Chinois ont, eux, employé le papier tordu en filament et doré à la surface dans le même but.

A vrai dire, aujourd'hui on n'emploie plus l'or pur en guimperie; par économie on use de fils d'argent que l'on dore par la galvanoplastie en les recourrant d'une mince couche, calculée pour résister au laminage, ou même tout simplement de fils de cuivre; il y a de l'or fin, de l'or $m i$-fin et aussi de lor faux dont on use fréquemment.

Largent tréfilé, laminé, enroulé sur une àme de couleur blanche par les mêmes procédés que lor, présente les mêmes variétés: fin, mi-fin et faux.

Au lieu d'enrouler très régulièrement les fines lames métalliques pour former des filis ou fils aussi arrondis et continus que possible, on peut, par la variété des supports employés, par leur préparation, par le travail mème de la guimperie, obtenir le frisé, la cannctille, etc., tous produits ayant des aspects plus ou moins contournés, gansés, torsadés, mats ou brillants, et dont on se sert non seulement dans le tissage, mais pour la broderie, les galons; ceux qui se prètent évidemment le mieux au mélange avec la soie sont les différents filés dont on use le plus généralement comme de trames; le passage entre les dents d'acier du peigne pour l'emploi en chaîne ne se fait pas facilement et présente de gros inconvénients. Il est préférable de s'arranger pour faire paraître cette trame de métal le plus possible à l'endroit du tissu et de ne la retenir au corps du tissu que de la façon la moins visible; elle donne de la sorte tout son effet et, par des flottés convenablement produits de cette même trame, les lignes du dessin se trouvent modelées; ce ne serait pas assez cependant pour obtenir toute la richesse et le détail nécessaires, et la plupart des brocarts comprennent des parties du dessin exécutées en broché, avec des fils de métal plus gros ou d'apparence plus marquée et variée, obtenus comme dit ci-dessus; ces parties de broché arrivent par certains artifices de tissage à s'enlever très en relief sur le fond, surtout dans les brocarts dits relevés; il va sans dire que tout ce travail d'exécution est fort délicat et nécessite de grands soins; il faut éviter tous les frottements brusques et intempestifs des fils métalliques contre ceux de soie qu'ils pourraient érailler et couper; ces précautions s'imposent encore plus lorsque l'on emploie comme trame la lame métallique simple telle qu'elle est au sortir du laminage et forcément tranchante sur ses bords, et qu'il s'agit de l'incorporer au milieu des fils de soie, bien à plat, pour obtenir des genres dits lamés, qui offrent une surface d'endroit tout à fait brillante et quelquefois même miroitante.

Assez nombreuses sont par suite les ressources dont on dispose pour varier l'aspect des brocarts. Si l'on en fabrique actuellement bien peu qui aient toute la beauté et la splendeur de ceux des siècles passés et de ceux qui servirent encore, sous 1'Empire et la Restauration, à composer la décoration de salles du Trône, dans les palais, ils'en tisse toujours quelque peu pour certains ornements d'église, ou tentures de prix. Aux robes souples et 
enveloppantes d'aujourd'hui ne conviennent guère les épais et lourds brocarts, étoffes pesantes se tenant toutes droites dans leur raideur forcée, qui s'adaptaient aux vêtements des époques de style; pour satisfaire les goûts actuels, quand la mode se porte vers les tissus oủ le métal se mêle à la soie, on est arrivé à les produire avec de l'or et de l'argent en fils extrêmement assouplis et plus encore à les fabriquer d'après le système du teint en pièces; on exécute par ce procédé, fort courant maintenant, comme nous l'avons dit pour des crêpes brochés or, des genres auxquels on aurait cru au premier abord son adaptation bien difficile et dangereuse; c'est ainsi que des damas lamés or, brochés or et jusqu'à des velours façonnés dans lesquels une trame d'or dessine le décor, maintiennent, ainsi conformé aux exigences modernes, ce prestige des soieries rehaussées de l'éclat des métaux précieux. 


\section{AUTRES SOIERIES FAÇONNÉES}

Il y aurait sans doute bien d'autres soieries façonnées à décrire, car on s'est ingénié à utiliser, comme nous l'avons mentionné, tous les différents genres d'armures ou de tissus unis comme fond, en y formant des dessins par la chaîne,

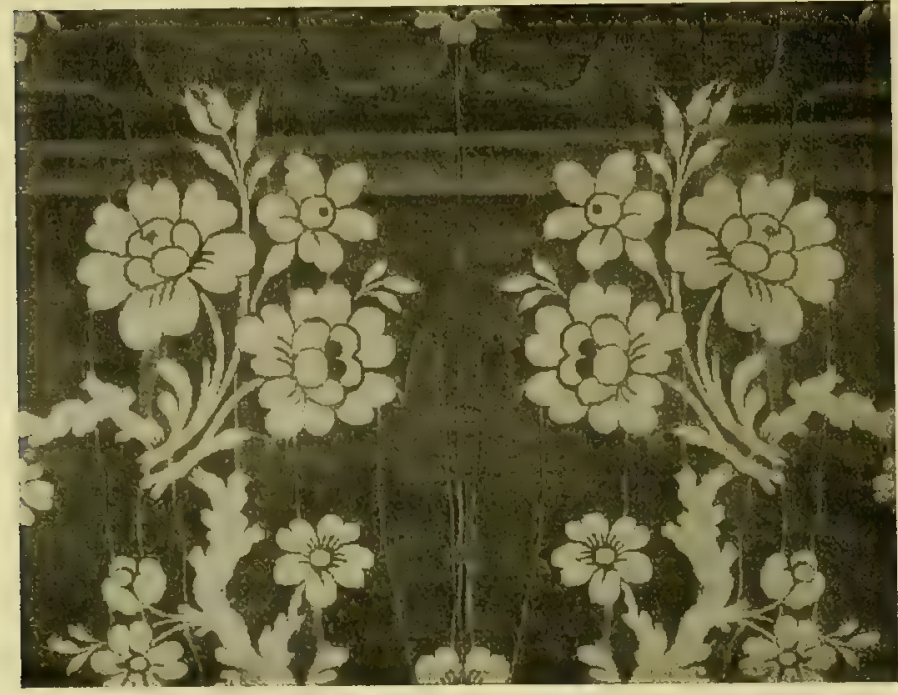

SOIERIE FAÇONNÉE MOIRÉE '. la trame ou les trames, en variant les aspects par l'emploi des matières, leurs colorations diverses, le traitement adopté, teint en fil ou teint en pièces, etc.

Bornons-nous à relever comme des catégories remarquables celles ou 1'on a combiné le façonné au jeu des fils donnant le dessin, avec des manipulations comme la moire, l'impression, et encore la broderie.

La première de ces associations, qui fut très employée au xvıI ${ }^{\circ}$, produit d'heureux effets en faisant se détacher le dessin sur des fonds moirés taffetas au gros de Tours et nécessite pour l'établissement de ce dessin qu'il soit exactement à retour, c'est-à-dire qu'il se reproduise identiquement, symétriquement sur chacune des moitiés de l'étoffe; il faut se rappeller en effet que la moire est produite par la pression très forte que subit l'étoffe pliée en son milieu sous la calandre; dans cette application, il est nécessaire que les parties du dessin coïncident les unes sur les autres dans ce pliage, sous peine de laisser une marque ineffaçable dans le fond moiré.

L'impression se combine, exécutée directement ou sur châ̂ne, ou encore ad hoc (termes dont nous verrons plus loin le sens) par des dessins unicolores ou multicolores, avec d'autres dessins façonnés par le travail du métier, pour des genres assez spéciaux et peu courants.

Enfin la broderie a quelquefois servi à compléter également le travail du métier dans certaines soieries dont le prix peut supporter cet enrichissement toujours assez dispendieux.

1 Communiqué par MM. Tassinari et Chatel. 


\section{BRODERIES SUR SOIE}

On a fait remarquer très justement que le mot broderie s'emploie avec deux acceptions: au singulier, pour signifier l'art de représenter sur une étoffe, et en relief généralement, par des fils passés le plus souvent avec le secours de l'aiguille, des figures et des dessins; au pluriel, pour désigner les ouvrages mêmes qui sont le produit de cet art de broder.

Il n'y a pas lieu de s'étendre ici sur la broderie en général, non plus que de faire valoir la grande importance de cette classe, remarquable entre toutes, des arts décoratifs du tissu et dont l'origine remonte à des temps si reculés; la broderie (ars phrygia, ars plumaria, opus anglicum, cyprense, etc.) a trouvé ses historiens érudits qui en ont décrit les très intéressantes manifestations chez les divers peuples, aux époques anciennes et modernes. Et, d'autre part, de nombreux commentateurs avisés ont étudié la valeur comparée ainsi que les différentes techniques des broderies.

De ces dernières, les seules que nous ayons à retenir sont celles exécutées sur soie, en les faisant compter à titre bien mérité parmi les différentes sortes de soieries à décor, et ceci bien qu'elles ne soient pas le résultat direct d'un tissage; il serait inutile du reste de faire cette mention autrement que sous une forme tout à fait succincte, a près la place de choix réservée à ces broderies dans les savantes études spéciales qui ont été consacrées à ce vaste sujet.

Il est tout naturel que l'on ait de fort bonne heure utilisé le fil de soie pour rehausser certains tissus de broderies; son brillant, sa solidité, sa souplesse, l'éclat nuancé de son coloris étaient autant de qualités de premier ordre qui le désignaient à cet effet. On le voit donc constamment concourir avec les fils d'or et d'argent, aux époques où la broderie fut tellement en honneur, à Byzance et en particulier au Moyen Age, pour décorer de riches tissus comme les draps d'or, le velours, le satin, etc., et former de magnifiques ornements d'églises, des vêtements somptueux, tout « raides de broderies », des étoffes pour tentures de prix, des gants, des aumônières et des œuvres comme la couverture du livre d'heures d'Isabeau de Bavière, citée comme un véritable monument de l'art de broder à cette époque.

La broderie était alors le travail extrêmement habile de nombreux ouvriers et ouvrières, travail régi par des statuts corporatifs sévères, et les traditions d'écoles de broderie en divers lieux, qui devinrent très renommées. « Broder fut même jusqu'à la fin du $\mathrm{XvI}^{c}$ siècle une branche sérieuse, estimable de la peinture; l'aiguille, véritable pinceau, se promenait sur la toile et laissait derrière elle le fil teint en guise de couleur, produisant une peinture d'un ton soyeux et d'une touche ingénieuse, tableau brillant sans reflet, éclatant sans dureté » (Fr. Michel).

Les dames de la plus haute noblesse, suivant un usage pratiqué de tous temps et remontant à l'antiquité, se livraient également au travail de la broderie. D’après un biographe de Catherine de Médicis, « elle passait, dit-il, 
fort son temps les aprés-disnées à besogner après ses ouvrages de soye oủ elle était tant parfaite qu'il était possible $\gg$.

Et Ronsard dit encore dans son ode à la Royne de Navarre, en la comparant à la déesse Pallas:

Vous d'un pareil exercice,

Mariez par artifice

Dessus la toile en mainct trait

L'or et la soie en pourtraict.

Le goût de ces travaux de l'aiguille, plus ou moins luxueux, plus ou moins habiles, ne devait point se perdre, malgré les défenses royales et peut-être à cause de celles-ci, un instant édictées contre le port des broderies dans les vêtements, et, au XVIII ${ }^{e}$ siècle, la broderie, qui avait été limitée d'abord à la toilette des princes et des personnes du plus haut rang, devint d'un usage presque général : les habits, les uniformes, les ornements d'église, les étendards, les housses de cheval, les meubles étaient brodés avec des fils d'or, d'argent, de soie, mème de lin ou de laine. La broderie s'établịt à Lyon comme une branche précieuse dindustrie; les broderies de Milan et de Venise étaient alors en grande renommée, mais leur excessive cherté en avait contrarié l'usage. Et les fabricants de Lyon « marièrent les chefsd'ceuvre de la navette avec ceux de l'aiguille », dit Roland de la Platière, « avec beaucoup d'habileté et de goût ». Vers I778-I780, ce genre de fabrication s'était si prodigieusement accru par une célébrité acquise dans le monde entier, tant sur les diverses parties des vêtements d'hommes et de femmes que sur les bas de soie, ornements d'église, étoffes d'ameublements, qu'il entretenait et faisait vivre, a-t-on affirmé, 20.000 personnes, au nombre desquelles se trouvaient plus de 6.000 brodeurs.

"Les fabricants, dit un auteur du temps, viennent de faire des étoffes à 600 francs l'aune pour habits d'homme et l'on n'est plus effrayé de ce prix excessif. »

Pour la composition de ces broderies, on avait recours aux plus célèbres dessinateurs; Philippe de Lassalle dui-même établit le dessin de plusieurs pièces remarquables, entre autres de cette très belle tenture qui fut souvent reproduite, toute brodée au point de chaînette sur satin blanc, à destination de Trianon.

Un autre artiste contemporain, dont le talent et le mérite incontestable ont fait citer fréquemment le nom à côté de celui de Philippe de Lassalle, Bony, né à Givors, près de Lyon, acquit une fort grande réputation en composant une quantité de dessins pour broderie de soies pour les robes, les gilets, les habits; il fut 1'auteur également de nombre de dessins de soieries façonnées exécutées au métier : velours, damas, lampas, etc.

Sous la direction éclairée et impeccable de ce maître, les ateliers lyonnais de broderie exécutèrent des pièces incomparables de goût, de délicatesse et d'élégance.

L'une d'elles mérite, sans doute, d'être considérée comme le chef-d'œuvre de Bony : c'est la tenture qu'il composa et fit exécuter, entièrement brodée 
au passé sur fond de satin, en soies de couleurs, en or et en chenille, pour le petit salon de l'impératrice Marie-Louise, à Versailles. Cette magnifique décoration murale, aussi remarquable par la valeur du dessin que par le mérite du travail, ne fut d'ailleurs jamais mise en place et, retrouvée intacte et dans toute sa fraîcheur au Mobilier National, elle a figuré dans plusieurs expositions pour le plus grand honneur de la broderie lyonnaise.

On brode encore sur soie maintenant à la main, d’après les anciennes

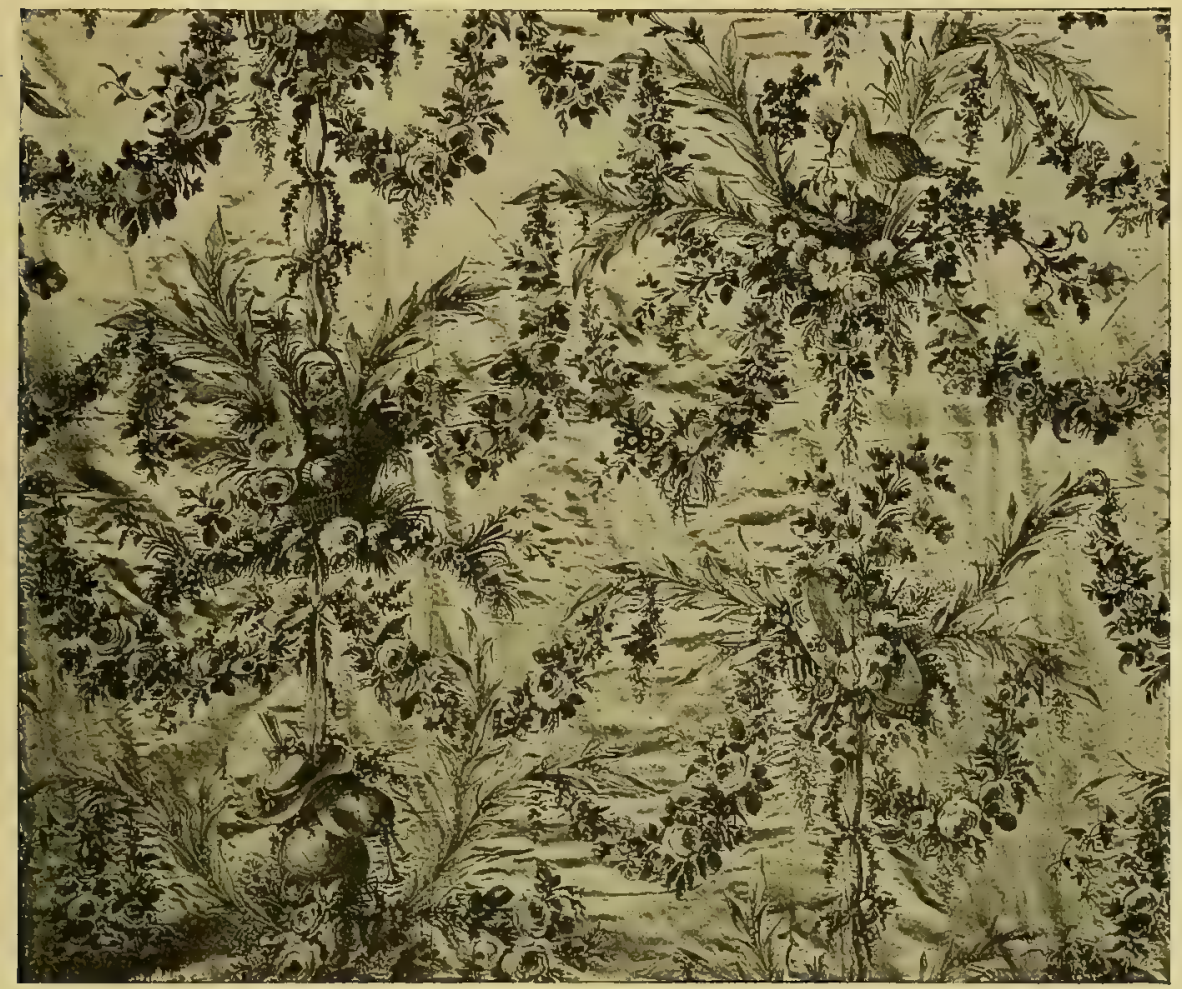

SolerIe BRodée (composition de Ph. de Lasalle, exécutée pour Trianon 1).

manières, en usant de toutes les ressources et de tous les points d'un art dont la technique fut poussée, il y a si longtemps déjà, à une variété tout à fait grande. C'est tantôt à l'aignille sur le tissu tenu sur le doigt, ou tendu, places par places, sur le léger cercle du tambour ou enfin avec le secours du métier à broder classique et bien connu, qui permet aussi d'exécuter commodément la broderie au crochet. Et l'on fait de la sorte maints ouvrages, écrans, menus accessoires élégants de toilette ou d'intérieur, aussi bien que l'on illustre de broderies faites en ateliers, des soieries pour robes et garnitures, certaines étoffes pour l'ameublement, des ornements d'églises, etc.

L'application de la broderie réussit particulièrement bien sur toutes les

1 Collection du Mobilier National. 
soieries légères, crêpes, gazes, mousselines, tulles, si répandues maintenant; le contraste sur un fond clair et plus ou moins transparent des dessins faits de points de soies de couleur, d'or, d'argent, enrichis d'agréments comme les paillettes, les perles, et d'autres variétés, fournit d'heureux effets et des formules décoratives nombreuses.

Il ne faut pas non plus manquer de dire que linvention et la mise en pratique de moyens mécaniques pour fabriquer des broderies ont beaucoup contribué à vulgariser leur emploi. Le métier suisse de Saint-Gall, dont les applications commencèrent par des broderies blanches, faites sur fond de toiles ou de divers tissus de coton, permet d'obtenir sur soie et avec de la soie des broderies plus ou moins compliquées.

Son ingénieux système repose, en principe, sur l'emploi du pantographe dont la pointe maniée par lourrier brodeur suit sur une carte spéciale oú ils ont été tracés, les points du dessin à reproduire et détermine en même temps les déplacements du cadre vertical qui porte l'étoffe tendue; après chacun de ces déplacements une série daiguilles ḋ deux pointes, entraînant dans leur mouvement les fils de soie qui ont été passés dans un trou pratiqué en leur milieu, traverse létoffe au moyen dune ingénieuse disposition de griffes doubles qui laissent ces aiguilles d'un côté du tissu et les reprennent de l'autre, comme autant de mains d'ouvrières brodeuses pourraient le faire; et l'on arrive de la sorte à broder à la fois plusieurs largeurs du dessin répété dans un ensemble de tissu qui peut atteindre plusieurs mètres de développement. L'économie de temps est évidente et ce qui étonne encore est de voir quel degré de perfection ce métier, habilement manié, peut donner dans ses résultats; aussi bien que des broderies lourdes ou légères d'un ou de plusieurs tons sont exécutées avec cette machine des broderies avec ajourages, festons, etc., et encore dautres faites sur tulles ou canevas auxquelles la composition du dessin, le genre du travail et divers artifices arrivent à donner l'aspect de véritables dentelles; il est vrai que ces derniers genres se réclament bien moins de la soie et que support et fil de broderie sont le plus souvent en coton. La soie artificielle cependant, dont nous avons parlé, s'est prêtée depuis quelque temps à des utilisations notablement importantes pour les usages de la broderie, mécanique surtout; son brillant spécial, très accusé, arec des reflets presque métalliques, la fait rechercher pour cet emploi, qui n'exige pas d'ailleurs une particulière finesse de fil, non plus qu une solidité très résistante comme pour le tissage au métier.

Il existe d'autres machines à broder, plus simples et ne donnant que des résultats bien plus limités; la mieux connue est une sorte de machine à coudre où l’étoffe supportée par un plateau mobile est entraînée par les déplacements que l'ouvrière donne à celui-ci, pendant qu'une aiguille mécanique coud sur le tissu un lacet, tresse, cordonnet ou bourdon, de soie ou d'une matière analogue. La broderie, ou plutôt l'imitation de broderie, se fait ainsi en suivant les contours du dessin indiqués à l'avance avec un poncif sur l'étoffe ou même sans dessin aucun, l'ouvrière ayant assez d'entraînement ou d'habitude pour reproduire de façon suffisante le modèle placé sous ses yeux.

Quelle différence existe entre ces travaux de broderie à bon marché et ceux des artisans orientaux encore pratiqués d'après des règles invariables, en accord avec les habitudes de patient et long labeur manuel, de pays où 
les traditions de la broderie sont séculaires. Les broderies de la Chine, du Japon et de l'Indo-Chine, par exemple, alors même qu'elles sont maintenant le plus souvent exécutées, en soie toujours, mais sur des fonds assez ordinaires, comme des satins tramés coton de provenance européenne, se rattachent directement par les dessins, les coloris et le mode de travail de l'aiguille, aux modèles admirables des mêmes provenances, fournis par les époques anciennes. Et ceux-ci comprennent entre mille autres choses: des robes de mandarins, des châles de crêpe de Chine, des kimonos, ornés de broderies faites avec un soin, une régularité, une habileté, que l'on n'a pas surpassés en Occident. N'oublions pas que des broderies de soie japonaises, de composition et d'exécution toute moderne, ont révélé en Europe, quand elles y furent exposées, autant d'intérêt dans le choix et l'arrangemeint des dessins, de charme dans les coloris, que de mérite resté surprenant dans la broderie elle-même, attestant un art qui s'est conservé dans toute sa pureté. 


\section{SOIES PEINTES, SOIERIES IMPRIMÉES}

Après 1e procédé de décoration des étoffes de soie, qui repose sur le tissage seul, par le jeu des fils, leur mélange en couleurs associées, leur combinaison avec l'or, l'argent, etc., ainsi que nous venons de le voir, la manière la plus usitée d'illustrer les soieries de dessins variés est certainement, à l'heure actuelle, l'impression, dont les exemples sont si répandus.

On pourrait définir limpression : une sorte de peinture sur étoffe, rendue pratiquement industrielle, et, à vrai dire, il n'est guère possible de séparer les deux choses peinture et impression, surtout lorsqu'on se reporte aux origines; l'emploi du pinceau chargé de couleurs, manié à la main, librement, ou bien en s'appliquant à suivre les premiers contours d'un dessin, déterminés le plus souvent, il faut le supposer, au moyen de modèles ou patrons, a-t-il précédé, suivi ou accompagné l'usage du bloc de bois dur portant un dessin gravé en relief, et imprégné d'un produit colorant? On en est réduit sur ce point à des conjectures sans pouvoir rien affirmer.

Mais l'Orient fut encore très évidemment le précurseur dans cette voie; le pinceautage, ou bien l'impression primitive, s'est exercé, nous apprennent les historiens, à des époques lointaines en Chine, au Japon et particulièrement dans l'Inde, et non seulement on sut dans ces pays artistement et habilement distribuer au pinceau ou autrement des couleurs à la surface d'un tissu à des places convenables pour former des dessins, mais, mieux encore, la connaissance des mordants chimiques empiriquement employés, des réserves ménagées à la cire avec une étonnante dextérité, permit déjà, le fait paraît indiscutable, de varier beaucoup les effets obtenus pour le meilleur résultat décoratif.

A côté de ces productions remarquables, quel fut très exactement le système employé pour limpression au Moyen-Age de ces curieux tissus de lin, de laine, mème de soie, qui sont conservés dans certains musées et catalogués comme étant de travail rhénan ou italien du XIV et du Xv ${ }^{e}$ siècle? Sans pouvoir trop préciser, on est fondé à croire qu'il fut celui du bloc, ou de la planche en bois, avec dessin gravé en relief, mais de façon assez rudimentaire, et qu'il resta sans grand développement; cependant, l'impression typographique, dont l'invention repose sur un principe analogue, avec l'usage de caractères de bois en relief, prenait, dès son début, à peu près au même moment, l'essor que l'on sait.

Il faut arriver au XviI siècle pour constater queỉ fut le très grand succès en Europe, en France particulièrement, des tissus exotiques, décorés comme dit ci-dessus et importés soit directement des grandes Indes, par vaisseaux, soit par caravanes traversant la Perse. D'où les dénominations, tantôt d'Indiennes, tantôt de toiles de Perse ou Perses, par lesquelles on désigna le plus souvent ces toiles, englobées généralement aussi sous le terme de toiles peintes. De cette époque et surtout à cause des prohibitions et des défenses si 
curieuses lancées par le Gouvernement contre ces tissus à la requête des fabricants de draps et de soieries, date leur vogue considérable, vogue à la faveur de laquelle plusieurs fabriques s'établirent en Angleterre, en Suisse, en Allemagne, en Alsace surtout, et enfin en France, quand la mode fut plus forte que tous les édits royaux et que cette fabrication devint permise ${ }^{1}$.

Pendant longtemps cependant, les toiles peintes des Indes eurent la supériorité de la meilleure exécution, de la beauté et de la solidité des couleurs sur les produits européens. Ceux-ci restèrent grossiers et communs jusqu'à ce qu'on fût parvenu à employer couramment et d'une manière perfectionnée dans les manufactures le procédé de l'impression avec des planches en bois portant le dessin gravé en relief, innovation qui conduisit plus tard à la planche plate et au rouleau en cuivre, gravés en creux; en même temps des recherches intéressantes avaient amélioré la connaissance des matières colorantes et permis d'arriver à l'éclat et à la durée des couleurs dites dès lors " bon teint ».

Oberkampf est le plus célébre parmi ces manufacturiers de la fin du $\mathrm{XvIII}^{\mathrm{e}}$ siècle; dessinateur, graveur, chimiste lui-même autant qu'habile imprimeur, i1 créa en i 760 la manufacture de Jouy-en-Josas, dont les produits devaient atteindre une renommée si prépondérante qu'on en est venu même peu à peu et trop facilement, à nommer toutes les productions de cette époque «toiles de Jouy », sans distinction de provenance.

Tous ces détails ne concernent exclusivement ou à peu près que l'art du pinceautage ou de l'impression appliqué aux seules toiles, c'est-à-dire cotonnades ou tissus de lin. Il semble bien que l'on songea assez tard à se servir industriellement des mêmes procédés pour décorer les tissus de soie. Car les soieries peintes à la main dont on relève l'exécution vers le milieu du $\mathrm{XVIII}^{\mathrm{e}}$ siècle, et pour certaines aux Gobelins, furent seulement des fantaisies assez coûteuses, exécutées par des artistes de talent d'après le goût du temps, qui portait à décorer de peintures les tissus et les objets les plus variés.

Cependant, un peu plus tard, une tentative fort curieuse d'organisation industrielle, à Paris, pour la production de soieries peintes et destinées à des robes, est celle dont le fameux aventurier Casanova, chevalier de Seingalt, avait eu l'idée sous Louis XV, comme il le raconte dans ses mémoires?. Il ne donne, du reste, aucun détail pratique sur ses procédés, affirmant seulement « qu’il avait toutes les connaissances chimiques nécessaires »; aussi, quand il parle de « l'impression » dont il usait pour reproduire sur les étoffes de soie " tous les beaux dessins que l'on exécute à Lyon par les procédés lents et difficiles du tissage et à des prix bien inférieurs », on est amené à croire, en raison de ce qu'il dit ailleurs des ouvriers qu'il employait à « peindre» les taffetas, gros de Naples, etc., que ce travail consistait bien à colorier au pinceau des dessins dont la forme générale, la silhouette, pouvait avoir été tracée au préalable, en noir probablement, soit avec des planches de bois gravées, soit avec des sortes de patrons découpés.

Quoi qu'il en fût, la manufacture de Casanova ne put ni prospérer ni se maintenir, cela par la légèreté et la prodigalité de son fondateur,

1 Voir : la Toile peinte en France, la Manufacture de Jouy, H. Clouzot.

- Mémoires de Casanova, t. III, chap. xviI-xviII. 
engagé dans trop d'entreprises diverses et malgré la précaution qu'il avait eue de se mettre sous la protection du prince de Conti et d'installer ses ateliers dans l'enclos du Temple, l'un de ces emplacements privilégiés d'alors, jouissant de toutes franchises et exempté de la juridiction corporative.

Des exemples de soieries peintes de la même époque des plus curieux sont ces taffetas, poults de soie et satins, conservés jusqu'à nous et qui manifestement apparaissent décorés par le pinceau manié à la manière chinoise. On sait toute l'influence que l'art chinois exerça dès le XVII siècle en Europe à la suite de l'engouement pour les productions apportées de la Chine à cette époque, et il paraît certain qu'on eut recours à l'ha-

bileté des artisans de cette contrée lointaine pour décorer à bas prix, vu la modicité de leurs salaires, des soieries qui leur étaient envoyées de France et sur lesquelles les effets de coloration, la manière dont la peinture est exécutée, dénotent cette origine, alors même que l'ordonnance du dessin paraît quelquefois se réclamer de l'inspiration et de la composition européenne. Il ne faut pas oublier non plus ces étoffes de soie dites chinées - on les nomme parfois Hlammées aujourd'hui

- très répandues et très goûtées au XviII ${ }^{\mathrm{a}}$ siècle.

Sans qu'on puisse
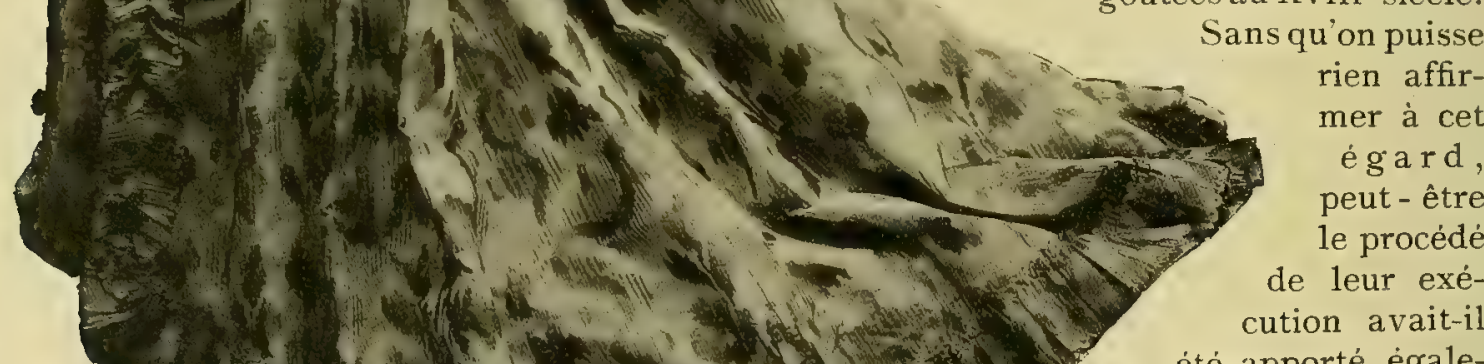

rien affirmer à cet é ga rd, peut - être le procédé de leur exécution avait-i1 été apporté également de la Chine comme leur nom semble lindiquer; en tous les cas, ce.genre de travail, dont la ROBE dE L'ÉPOQUE dE LOUIS XV EN tAFFETAS CHINÉ.

pratique et le tour de main sont aujourd'hui entièrement perdus, comportait des opérations longues, multiples et si minutieuses quion peut, avec vraisemblance, en attribuer l'invention à des artisans lents et patients comme l'ont toujours été les Chinois. 
Ce procédé dit du chiné à la branche ou par branche était une manière de faire des dessins sur la chaîne d'un tissu avant sa fabrication définitive; il reposait sur un système de teinture partielle, par endroits déterminés à l'avance, des fils de soie de la chaîne, selon les indications et les coloris du dessin tracé et peint d'abord sur papier réglé spécial; ces fils étaient séparés en flottes ou branches, de grosseur et de longueur convenables; après un ensemble de minutieuses mesures préparatoires, cette teinture ou mieux ces teintures successives s'opéraient une fois que les parties des flottes qui ne devaient pas recevoir de la couleur avaient été recouvertes par des ligatures très serrées au parchemin et à la ficelle, quitte à recommencer lopération pour la même flotte autant de fois qu'elle avait de couleurs différentes à recevoir.

Enfin, tout ce travail exécuté et les flottes une fois teintes remises à côté les unes des autres sur le métier avec un repérage qui mettait en place les parties colorées, comme il convenait pour composer le dessin, il ne restait plus qu'à tisser avec une trame de la nuance du fond choisi, en général blanc. Le tissage donnait aux étoffes chinées leur caractéristique très typique et reconnaissable, le dessin étant coupé par touches de couleurs bien séparées, à droit fil dans le sens de la longueur, et légèrement frangées ou follcttées au contraire à leurs extrémités.

Bien qu'on ait retrouvé l'énoncé des règles auxquelles se conformaient les chineurs on a peine à comprendre comment ils pouvaient obtenir autant de régularité dans l'exécution de ces tissus qui furent très en honneur, aussi bien pour les robes que pour la tenture et l'ameublement; et l'on reste étonné de la complication des dessins, de l'éclat des coloris que montrent certaines soieries chinées : taffetas, satins ou velours. Dans le cas du velours en effet, intervenait une complication nouvelle, celle de la diminution considérable de longueur au tissage des fils formant le poil du velours, l'embuvage, dont nous avons parlé en temps voulu; d'où l'obligation pour le chineur d'agrandir le dessin en conséquence, d'après un calcul donné par l'expérience pour obtenir finalement le dessin dans la forme et les dimensions prévues par l'esquisse.

Nous verrons bientôt que ce procédé compliqué et difficile a été abandonné et remplacé avantageusement par celui de limpression sur chaîne.

Des exemples frappants de toutes ces difficultés vaincues sont donnés par certains velours chinés, conservés au Mobilier National; commandés par Napoléon $I^{\text {er }}$, auquel plaisait particulièrement, paraît-il, ce genre d'étoffe, pour l'ornement des appartements de ses palais, ils furent exécutés à Lyon où l'art du chinage s'était toujours conservé. Et par exemple devant la tenture en velours chiné, destinée à la chambre de l'Empereur à Fontainebleau, au dessin de grandes proportions, aux tons vifs et nombreux - contesterait-on d'ailleurs la valeur artistique du résultat — on n'est pas surpris d'apprendre que cette tenture a coûté I 68 francs le mètre et nécessité deuxannées pour son achèvement! (E. Dumonthier, les Etoffes napoléoniennes.)

Aujourd'hui, chiner la soie de même que le coton ou la laine, ne signifie pas autre chose que préparer ces textiles en flottes, en les teignant, ou en les tachant pour mieux dire, d'une ou plusieurs couleurs, à des places régulières ou irrégulières, sans aucune préoccupation de dessin. 
Et l'on emploie (on a employé surtout fréquemment vers le milieu du $\mathrm{XIX}^{\mathrm{e}}$ siècle), les fils ainsi tachetés ou jaspés, pour former sur des fonds unis des rayures, ou des filets chinés, agréments qui s'enlèvent de façon intéressante.

Après le considérable développement qu'avait pris en France, comme nous l'avons déjà noté, à Paris, à Rouen, Mulhouse et quantité d'autres endroits l'impression sur tissus de coton, de lin, puis de laine, on serait en droit de penser que les mêmes procédés furent utilisés dès ce même moment

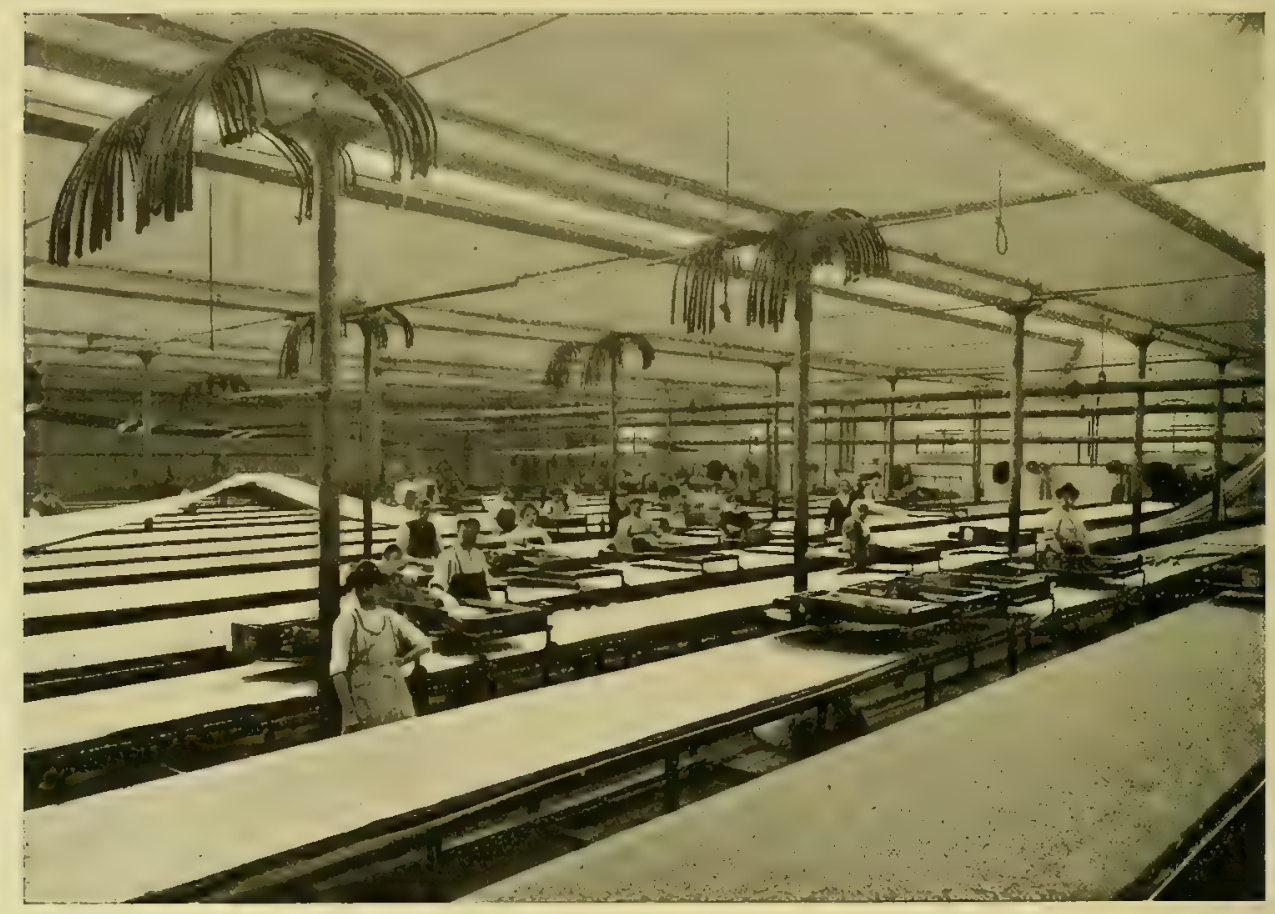

ATELIER D IMPRESSION DE SOIERIES 1.

pour décorer les étoffes de soie; on n'en relève des exemples cependant que d'une façon assez restreinte et il en fut ainsi certainement à cause des résistances corporatives, opposées par les fabricants et ouvriers façonniers en soie, redoutant la concurrence.

Ce n'est guère avant 1816 que la fabrication des soieries imprimées prit à son tour une marche vraiment industrielle, sous l'impulsion de fabricants 1yonnais avisés et inventifs, comme Revilliod et Depouilly entre autres, et que fonctionnèrent des manufactures qui joignirent à l'impression des indiennes celle des tissus de soie. Une des plus anciennes fut celle exploitée d'abord par Perregaux puis par la famille Brunet-Lecomte de père en fils, à Jallieu, près de Bourgoin; elle devait devenir une des fabriques d’impression les plus réputées pour le mérite et le goût de sa production.

1 MM. H. Brunet-Lecomte et Cir, Jallieu. 
Aujourd'hui,. les fabricants de soieries ne sauraient se passer des ressources décoratives que l'impression met à leur disposition et que ses procédés améliorés ont encore rendues plus nombreuses.

C'est à des étoffes de soie unies, sans difficultés de tissage, que l'impression s’applique le plus souvent et c'est d'une grande commodité en plus, de pouvoir utiliser par ce moyen des étoffes souvent fabriquées à d'autres fins dont l'aspect et le caractère se trouvent de la sorte transformés, renouvelés.

Qu'il s'agisse d'impression directe, faite sur le tissu uni tout terminé, ce qui est la façon la plus générale de procéder, ou d'impression sur chaîne,

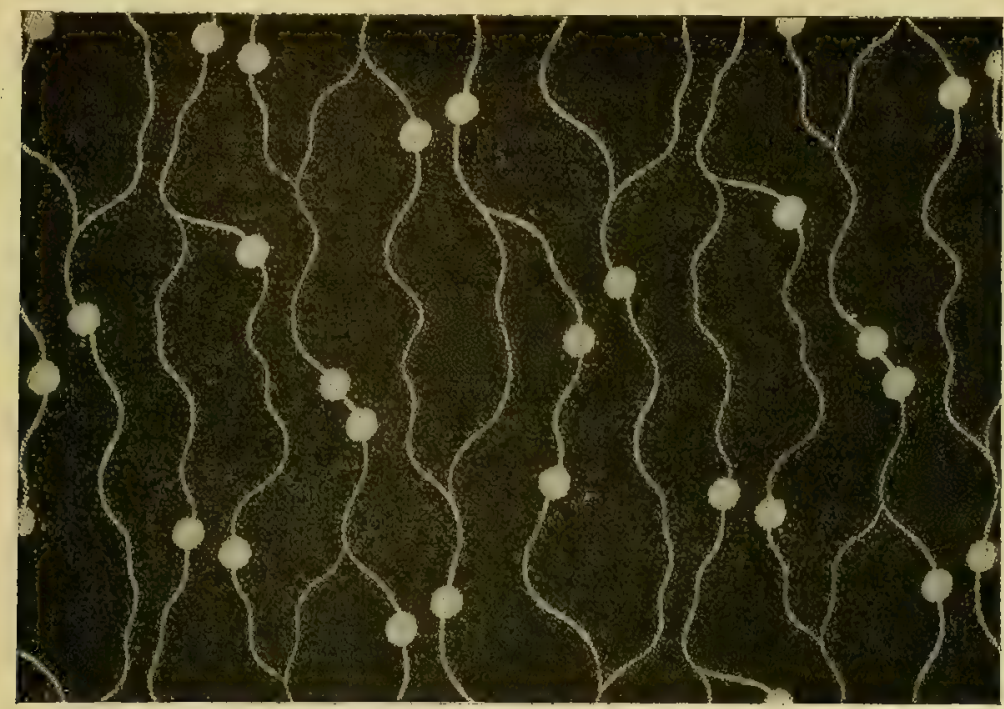

TYPE D'IMPRESSION, PAR ENLEVAGE.

système qui a détrôné 1'antique chiné dans la manière de préparer le dessin sur les fils avant le tissage définitif, le travail peut s'exécuter maintenant soit à la main ou à la planche, soit mécaniquement; c'est-à-dire la plupart du temps, au rouleau.

Les machines à imprimer modernes mues par la vapeur, ressemblent fort peu à la máchine employée à Jouy, si le principe en est à peu près celui de la machine à rouleau ayant succédé à celle à la planche plate, qui fut la première employée. Un ou plusieurs larges rouleaux de cuivre en nombre correspondant à celui des couleurs du dessin, gravés en creux par des procédés certes plus expéditifs que ceux d'Oberkampf, sont disposés de telle sorte, sur un bâti métallique, qu'ils pressent d'un côté contre le tissu mécaniquement entraîné, tandis que de l'autre, ils sont garnis et pour ainsi dire encrés, en quantité convenable, de la couleur choisie, à l'état de liquide assez épaissi. Un règlage minutieux s'impose, mais une fois qu'il est opéré on peut imprimer en quantité indéfinie le même dessin, au même coloris bien entendu.

On se sert encore d'une machine inventée par Perrot en 1839 , appelée pour cela perrotine, et dont le systeme mixte comporte un dispositif qui permet de 
garnir de couleur des planches spéciales gravées dans la largeur du tissu et de les imprimer à la suite les unes des autres sur ce tissu qui est entraîné au fur et à mesure, tous ces mouvements étant réglés par une commande mécanique.

L’impression mécanique est celle qui convient naturellement pour les soieries de genres courants et de prix bas ou moyens; contrairement à ce qui a lieu pour les cotonnades par exemple où l'on arrive très bien à exécuter à la machine des dessins compliqués et chargés de nombreuses couleurs en raison du grand débit qui permet une production intense; comme la plupart du temps, les métrages à faire, ne couvriraient pas les frais de gravure, et pour d'autres raisons de métier, on se contente de décorer au rouleau les tissus de soie avec des dessins à effets limités, comportant un petit nombre de teintes dans le coloris; trés souvent on use de simples pois ou de motifs dune couleur unique comme pour la vaste catégorie des foulards, twills et autres légères soieries pour robes d'été, traitées en enlevage ou en application auxquelles la mode réserve ses faveurs par périodes alternatives, en assurant à ces moments le travail de nombreux ouvriers imprimeurs.

L'impression par enlevage ou à réserves est un curieux procédé connu et pratiqué, nous l'avons dit, à peu près de tous temps; il consiste, pour obtenir par exemple, un effet, un pois blanc sur un fond bleu marine, à imprimer sur le tissu blanc et aux places convenables un mastic résineux, composition pâteuse spéciale, qui sèche rapidement à l'air et devient insoluble dans l'eau. La pièce de tissu étant ainsi préparée, on la plonge dans un bain de teinture; la couleur se dépose sur le fond, à l'exception des points couverts par le mastic. Quand on enlève ensuite ce mastic par un dissolvant approprié comme la benzine, les parties du tissu qui ont été préservées de la teinture apparaissent en blanc.

Dans l'application, au contraire, c'est le fond dont on applique la couleur sur le tissu blanc par le moyen d'un rouleau gravé en conséquence, qui laisse sans impression les parties, pois ou dessins, qui doivent se détacher en blanc sur ce fond.

Une autre manière encore d'arriver au même résultat c'est de teindre tout d'abord le tissu avec des colorants spécialement choisis, puis de détruire cette teinture par places, de la ronger par l'impression de substances chimiques, dites rongeants, et qui soit employées seules, donnent l'effet blanc sur un fond coloré, soit mélangées à une substance colorante, permettent de substituer cette couleur même dans les endroits du dessin à la première obtenue par la teinture, ce qui donne en fin de compte un dessin de couleur sur un fond d'une autre couleur.

Cette ingénieuse façon de procéder, dont les applications se sont beaucoup développées assez récemment, n’a pas été utilisée seulement dans l’impression à la machine, mais a permis également d'étendre notablement le champ d'action de l'impression à la main.

Pour celle-ci, la chose d'importance, c'est la gravure du dessin, c'est-àdire l'exécution des planches de bois portant les différentes parties et couleurs de ce dessin en relief.

L'esquisse, bien entendu, a été faite au préalable, d'après les règles générales qui président à l'établissement des dessins pour soieries façonnées tissées; il faut encore que le dessinateur se préoccupe de ménager les raccords 
pour la répétition sans fautes ni discordances des motifs dans l'ensemble du tissu; il doit aussi tenir compte d'un rapport imposé dans le cas présent par les dimensions que l'on peut donner pratiquement à ces planches faites d'un bois dur et renforcé de plusieurs épaisseurs collées, qui sont par suite assez lourdes et cependant doivent rester maniables pour la commodité du travail. Aussi, lorsque l'on veut exécuter des dessins de grandes proportions, est-on souvent obligé de graver des jeux de planches combinés dont chacun correspond à une seule des parties du dessin.

Pour peindre une esquisse destinée à l'impression, il faut évidemment se servir de tons séparés ou comptés, dont le nombre est déterminé à l'avance par les convenances du dessin, en raison de son importance et du prix prévu pour l'étoffe, puisque chacun de ces tons doit être reporté à peu près toujours isolément sur une planche correspondante et qu'on ne peut imprimer, à la main, qu'une seule couleur à la fois.

Le travail délicat qui consiste à trier les différentes couleurs et à les reporter chacune à leur tour sur les planches, se nomme la mise sur bois; au moyen d'un papier à calquer fort, dit papier glace, on relève soigneusement sur l'esquisse les différentes parties d'une même couleur, puis on les reporte au trait sur la surface de la planche de bois bien aplanie et polie. C'est ensuite le travail de la gravure proprement

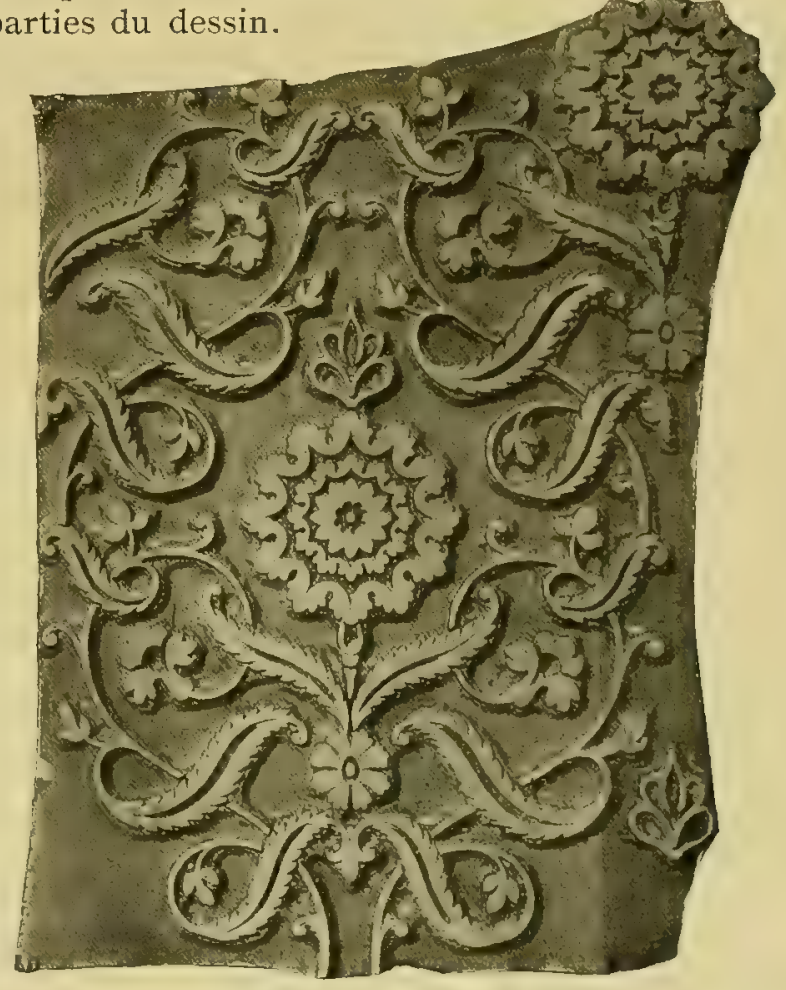

Planche D'impression GRAVÉE EN RELIEF SUR BOIS !.

dite: avec des outils appropriés, l'ouvrier creuse le bois délicatement tout autour de ce que l'on a marqué comme devant rester en relief et façonne patiemment ces reliefs; il faut pour cela beaucoup de précaution et d'habileté, surtout quand on doit ménager des parties de bois assez fines; quand celles-ci deviennent par trop déliées pour que le bois puisse offrir une suffisante résistance, on est obligé de lui substituer, à ces endroits, de minces lamelles de cuivre, qui sont contournées à la pince et enfoncées ensuite dans la planche en les ajustant à la hauteur convenable.

D'autres fois, pour certaines parties du dessin ou certains dessins en entier, on est amené à les former d'un alliage métallique dit plombine que l'on coule dans une matrice creusée d'après le dessin avec une lame rougie au feu ou bien le crayon à pyrogravure, dans un bloc de bois dur, le cachet.

1 Communiqué par M.M. H. Brunet-Lecomte et Cie. 
Une fois la gravure exécutée pour chacune des couleurs du dessin, on se trouve en possession d'une série de planches auxquelles sont parfois adaptés des repéres destinés à rester à peu près invisibles, sortes de légères pointes métalliques dites picots; ou bien encore on use d'une planche spéciale dite de touche, systèmes dont nous verrons bientôt la nécessité.

Reste à garnir chacune de ces planches à leur tour de couleur indiquée par l'esquisse ou le coloris général.

On se sert aujourd'hui pour l'impression sur soie des mêmes produits colorants que ceux usités pour la teinture; les gammes en sont fournies exclusivement par des combinaisons chimiques minérales et ne sont plus empruntées, comme dans le début, à des substances extraites de certains végétaux.

On les emploie, par exemple, à l'état semi-liquide, et presque pâteux, en les épaississant avec des gommes; il est nécessaire en effet d'éviter qu'à leur application sur le tissu, ces couleurs ne s'étendent en coulantet en faisant tache.

Après préparation au laboratoire on applique ces couleurs à la brosse sur des châssis spécialement établis à cet effet et qui sont comparables aux tampons encreurs de ces timbres en relief employés dans le commerce. La préparation de ces châssis est importante et demande des soins pour que la couleur s'y trouve en quantité juste convenable et autant que possible constante pour imprégner les reliefs des planches lorsque louvrier imprimeur pose légèrement celles-ci à leur surface avant de les appliquer sur le tissu à imprimer qui a èté bien tendu et fixé à l'avance sur des tables garnies de feutre épais.

C'est un curieux et intéressant spectacle que celui d'un atelier d'impression, pièce d'ordinaire vaste et bien éclairée, oủ l'on voit le long des longues et larges tables parallèles, les ouvriers manier avec dextérité les planches, en prenant chacune d'elles à son tour pour la poser d'abord sur le châssis roulant sur rails à ses côtés et correspondant à la couleur qui esten train, puis la placer avec précaution mais sủreté sur l'étoffe, juste à la place voulue en juxtaposant, superposant, rentrant les différentes couleurs dans un ordre donné; travail de marqueterie précise qui reconstitue au total le dessin primitif de l'esquisse, et pour lequel le repérage adopté pour les planches est, on le voit, indispensable, en plus d'une habitude professionnelle bien assurée par un long exercice.

Une partie difficile de l'impression est aussi de savoir, pour l'ouvrier, faire une bonne fourniture, c'est-à-dire d'apprécier pour chaque planche la quantité de couleur qui lui convient d'après son importance et sa place et encore d'après le genre du tissu : tout ceci pour qu'il y ait de la couleur juste ce qu'il faut et qu'elle ressorte très unie.

Et, du reste, le travail de l'impression à la planche se complique encore par de nombreuses recherches d'exécution : dessins à grand nombre de couleurs, ou de mains - en plusieurs parties, - à fonds, c'est-à-dire qui comportent, pour faire ressortir le dessin, 1 impression de la couleur du fond avec des planches spéciales, - dessins à placements particuliers sur le tissu : bas de robes, bordures d'écharpes, encadrements de châles, etc. - dessins imprimés ad hoc, c'est-à-dire uniquement sur certaines parties du tissu prévues au tissage - dessins traités en ombrés, - dessins imprimés avec de loo (poudres de bronzes colorés), etc., etc. 
Mais l'ouvrier le plus habile n'est en tous cas presque constamment, au point de vue du coloris, qu'un exécutant de la besogne qu'on lui a tracée par avance en indiquant les couleurs qu'il doit employer ainsi que la hauteur des tons, le tout pris dans des gammes bien référencées et numérotées. Ce choix est fait par le coloriste avec la valeur importante que lui donne dabord le goût personnel de celui-ci, autant si l'on veut qu'un entraînement soutenu et une grande expérience de métier; c'est un travail fort délicat et dont le réel mérite

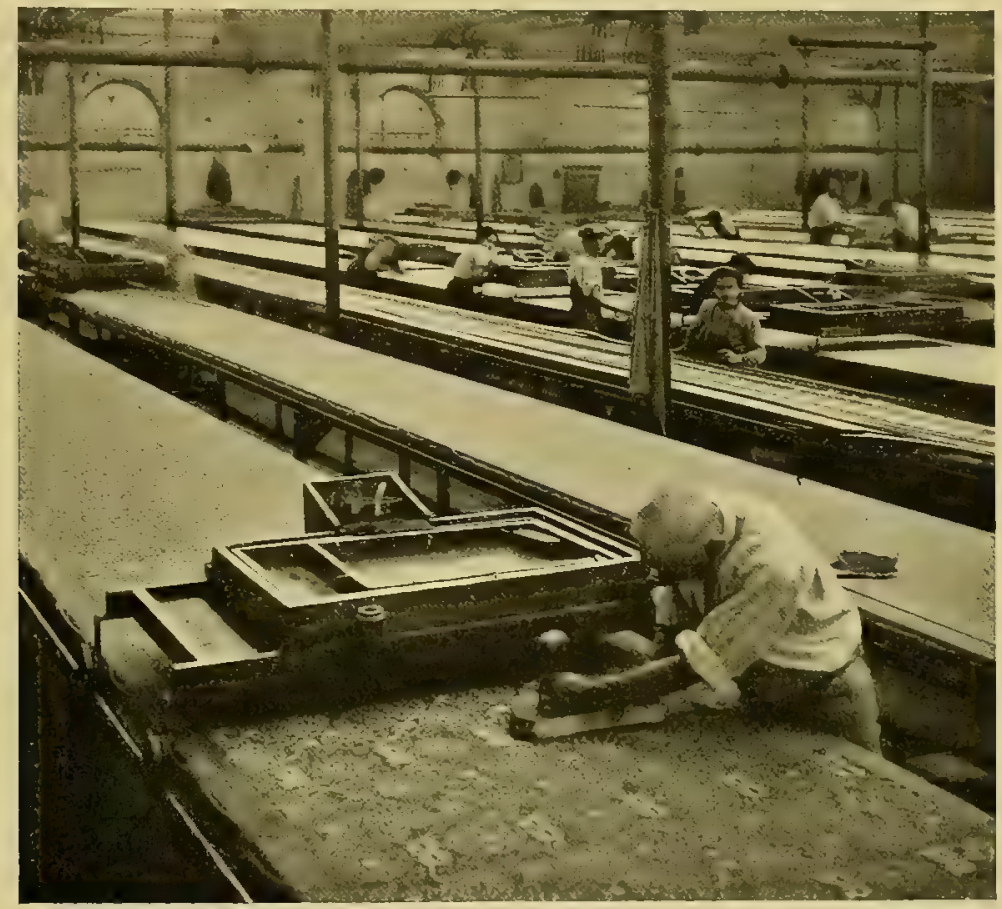

OUVRIER IMPRIMEUR APPLIQUANT SUR L'ÉTOFFE

LA PLANCHe QU'IL VIENT DE GARNIR DE COULEUR SUR LE CHASSIS PLACÉ A SA DROITE'.

n'est pas seulement de reproduire du mieux possible le coloris employé par le dessinateur pour l'esquisse, mais surtout d'en créer, pour les besoins commerciaux de l'assortiment, des variantes diverses qui sont comme des traductions, plutôt des interprétations renouvelées de lidée première. Cependant, avec les donnẻes qu'il puise dans son imagination et son éducation. décoratives avec les inspirations de sa fantaisie, le coloriste doit concilier les nécessités de l'exécution, prévoir l'effet des associations de tons, leur rendement sur le tissu, une fois ces opérations terminées, comme certaines de leurs superpositions ou retombées; il faut encore tenir compte du genre mème du dessin qui se prête plus ou moins aux oppositions de couleurs franches aux

1 MM. H. Brunet-Lecomte et Cie, à Jallieu. 
combinaisons de teintes fondues et dégradées, aussi bien que de l'e mploi auquel est destiné le tissu que l'on s'occupe d'imprimer.

On a certainement fait en impression sur soie de réels grands progrès dans le moment présent en sortant des anciens errements de coloris, un peu toujours les mêmes et de valeur parfois discutable. Comme il est facile de le voir en jetant les yeux autour de soi, au moins pour un certain ordre de productions, le niveau atteint est véritablement très artistique et dénote chez leurs auteurs autant de goût qu'une grande compétence de spécialistes.

Ainsi, dans l'emploi de ces produits chimiques rongeants, dont nous avons parlé plus haut et que lon incorpore aux couleurs dimpression, de façon à pouvoir les substituer à leur place à la teinte déjà donnée et spécialement préparée, un tour de main attentif guidé par certains moyens pratiques s'impose, car les couleurs obscurcies par ce mélange n'apparaissent pas sur la table d'impression avec la hauteur et l'éclat qu'elles auront par la suite; lexpérience avisée est un excellent guide comme en nombre de cas du reste.

Une fois que le tissu est imprimé dans toute la longueur qui est étendue sur la table et qu'il est assez sec, cotte longueur est enroulée à l'une des. extrémités de la table sur un rouleau à cet usage, tandis qu'une nouvelle longueur de tissu se trouve du mème fait étendue pour être imprimée à son tour et ainsi de suite jusqu'à la fin de la pièce.

Celle-ci, terminée, est soumise dans la plupart des cas à l'opération importante du fixage des couleurs, qui leur donne la solidité à l'air, à la lumière, même à l'humidité et en outre développe toute leur fraîcheur. On fixe les pièces imprimées en les soumettant à l'action de la vapeur sous pression dans une cuve close aménagée á cet effet. Sous cette action il se produit une modification intime des produits colorants employés, qui assure leur pénétration dans la fibre même des fils de soie et en même temps communique à l'impression la solidité requise.

Après, c'est le lavage à grande eau courante pour enlever les excés de substances employées et dépouiller la soie de tout empâtement; puis le séchage dans de grandes chambres oủ circule de l'air chaud; ce séchage a été précédé de l'avivage, nécessaire, comme après la teinture, pour redonner à la soie son toucher accoutumé, nerveux et craquant et lui permettre de prendre convenablement l'apprêt; car, il faut naturellement réapprêter l'étoffe après l'impression pour qu'elle ait à nouveau l'aspect, la présentation indispensable à sa mise en vente et à son emploi. Et ce sont, il ne faut pas l'oublier, des soieries teintes en pièces, qui s'accommodent exclusivement ou à peu près de ces manipulations humides.

Voilà, semble-t-il, un ensemble respectable dopérations pour réaliser limpression directe sur le tissu! Plus compliquée encore est l’impression sur chaîne que nous avons déjá nommée plus haut à propos des soieries chinées.

Ce procédé consiste à imprimer le dessin sur les fils de soie de la chaîne de l'étoffe que l'on se propose de fabriquer et avant cette fabrication, quelque paradoxal que ceci puisse paraître au premier abord. En réalité, pour parvenir à ce résultat, on tisse une première fois, avec les fils de chaîne, choisis et disposés comme à l'ordinaire pour le tissage et préparés avec un soin particulier au décreusage, un premier taffetas à peine consistant, avec une trame légère et espacée; ceci à seule fin de maintenir la chaîne juste assez pour 
qu'elle puisse supporter l'impression, sans que les fils en soient dérangés ou embrouillés. On imprime alors cette chaîne, ce tissé, avec la délicatesse et les précautions que cela comporte, on lave, on fixe, etc., et puis on replace sur le

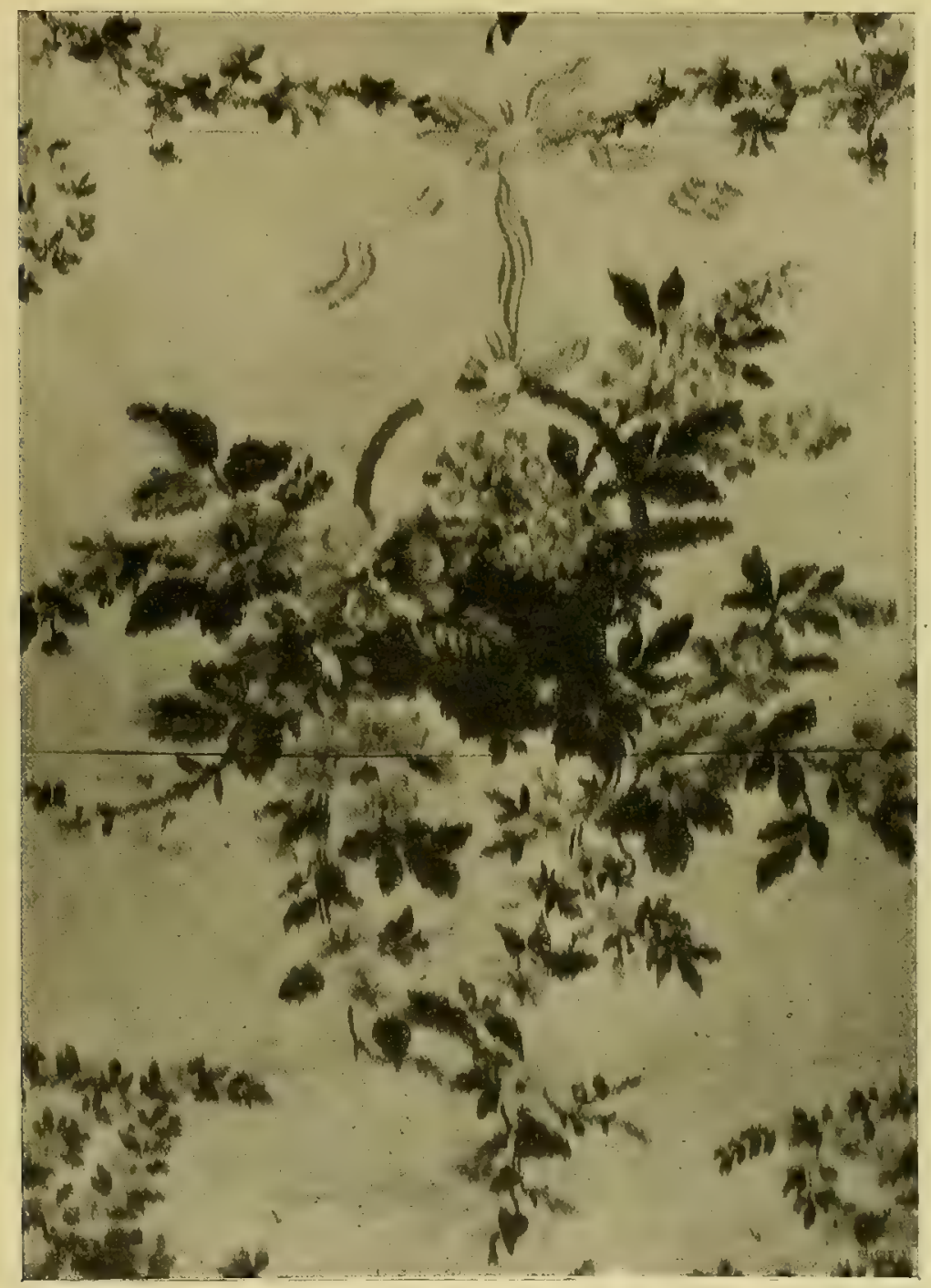

Solerie D'AMEUBLEMENT, IMPRIMÉe SUR CHAINE '.

métier. Le travail du tissage définitif s'exécute en détissant d'un côté du métier au fur et à mesure et en enlevant la première trame inutile, pour la remplacer de l'autre par une nouvelle trame distribuée cette fois comme dans le tissage ordinaire.

1 Communiqué par MM. Tassinari et Chatel. 
Avec des soins, le dessin apparaît sur l'étoffe sans déplacement appréciable dans sa forme générale; tout au plus présente-t-il sur les bords cet aspect légèrement fouctté du chiné, qui en estompe et en adoucit les contours et donne à lensemble une note particulière souvent charmante et que vient encore varier heureusement la couleur de la trame qui sert au second tissage.

La mode a fréquemment accordé ses préférences aux soieries ainsi décorées, comprenant des taffetas, des poults de soie, des satins unis, quelquefois des damas façonnés dont la contexture cependant se prête évidemment moins à la réussite du genre; enfin des gazes, de nombreuses sortes de rubans et mème des velours, toutes étoffes destinées soit à la toilette féminine, soit aux usages de l'ameublement.

L'impression en général offre donc, on le voit, pour la soie, mieux encore peut-être que pour le coton où son application industrielle est parvenue au développement si considérable que l'on sait, une grande variété, si elle n'est pas indéfinie, de moyens d'ornementation pour tous les goûts et presque à tous les prix. Ici, la qualité, le genre, la diversité des soieries recevant limpression interviennent pour beaucoup dans le résultat final; tel dessin imprimé sur une mousseline transparente, un crêpe de Chine vaporeux, un tulle léger, une gaze ajourée ou encore mêlée de fils d'or ou d'argent, se trouvant véritablement modifié, transformé au point quelquefois d'être à peine reconnaissable.

Le besoin de nouveauté servant d'aiguillon à cette recherche, on s'est ingénié du reste à poser des problèmes combinant le tissage et l'impression et à les résoudre élégamment.

C'est ainsi que fut créé le Velours au sabre: sur les fils d'un satin d'une armure particulière, par l'impression sur la chaîne ou directement sur des parties de ce satin tout tissé, bandes ou motifs détachés s'enlevant sur un fond différent, on a imprimé le dessin; l'étoffe est alors confiée à une ouvrière exercée qui, au moyen d'une lame d'acier acérée et coupante, maniée à la main, découpe petit à petit par le milieu les points assez larges formés par les fils du satin; ceux-ci se redressent, et le tissu dans ces endroits prend l'apparence chatoyante du velours, ce qui ombre les tons du dessin et les fond très heureusement entre eux. C'est d'une exécution forcément assez longue et coûteuse; aussi ce procédé n'est-il guère usité que pour des étoffes d'un certain prix, que justifie bien leur charme particulier, très supérieur à celui des genres que l'on obtient en imprimant directement sur le poil d'un velours tout fabriqué, par les moyens ordinaires.

Il $y$ a quelque rapport entre ces velours au sabre et ces curieuses productions du Japon, très répandues maintenant dans le commerce, sortes de tableaux de paysages, animaux, fleurs, etc., peints sur un tissu qui est un velours de coton frisé et découpés ensuite par places pour former le velouté, avec une habileté surprenante.

Il est vrai que l'on possède aussi le moyen, à l'imitation encore du chiné, d'imprimer sur chaîne les fils formant le poil d'un velours et de tisser ensuite celui-ci. Mais il y a là une telle complication, résultant de l'établissement de planches gravées spécialement, en proportion du retrait subi par les fils au tissage, et puis des soins minutieux à donner à la fabrication, que 
n'ont guère été faites de cette manière que les belles choses destinées à des expositions, restant bien en dehors de la moyenne courante.

Enfin, il est curieux de voir que l'impression à la planche issue, nous l'avons vu, du primitif pinceautage, a servi de nos jours à imiter la véritable peinture sur soie. On a façonné pour cela des soieries en les brochant par exemple de motifs à fleurs ou d'autres ornements, en prenant bien soin d'en assurer la régularité. Puis, au moyen de petites planches gravées ad hoc, garnies de couleurs soigneusement préparées et dégradées, on a coloré presque un à un sur le tissu ces seuls motifs. C'est à s'y méprendre lorsqu'on voit des étoffes ainsi décorées et à croire qu'elles ont été réellement peintes au pinceau.

Il est vrai de dire que dans cette sorte de lutte de la planche gravée contre le pinceau celui-ci n'a pas désarmé. On sait toujours très bien peindre sur soie, et non seulement à l'occasion de ces travaux quelque peu menus mais artistiques de l'éventail, de l'écran et de mille autres objets du même ordre élégant, mais aussi quand la mode l'ordonne pour illustrer des damas, des brochés, des mousselines par des touches légèrement appliquées à la main de couleurs spécialement préparées.

La pratique de cette peinture sur soie s'est exercée de façon si courante à certains moments qu'elle en devint presque industrielle.

Et puis, nouvelle concurrence, est arrivé récemment l'aérographe; cette machine, établie d'après un ingénieux principe, utilise la cou-

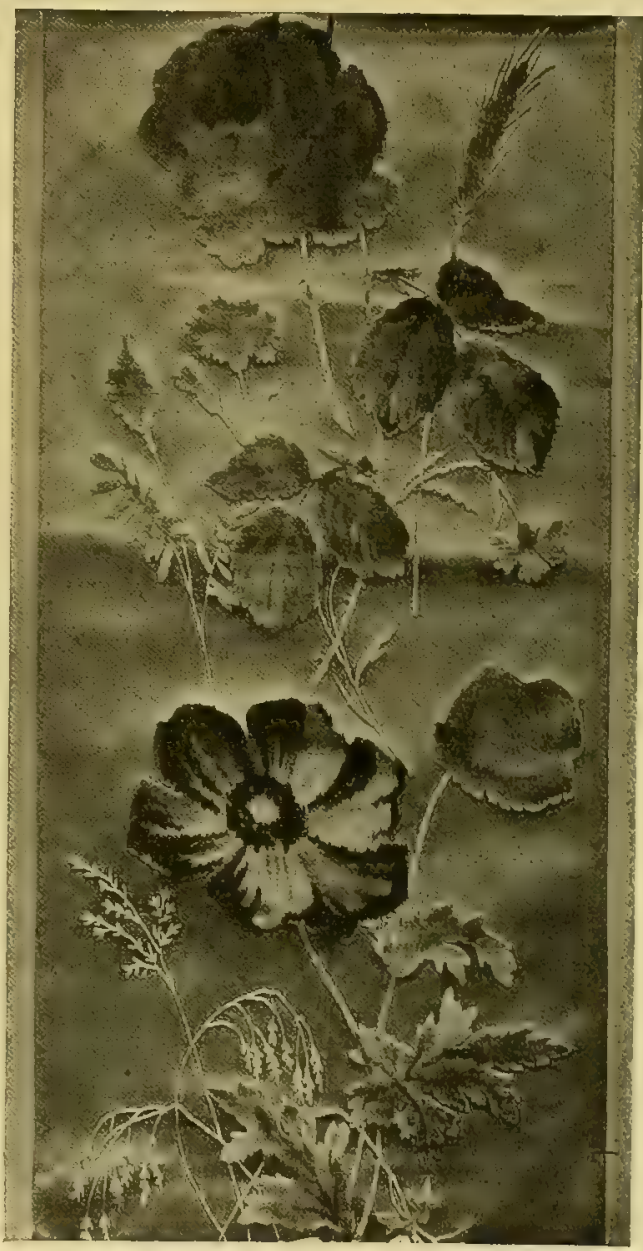

RUBAN FOND SATIN

AVEC DÉCOR DE VELOURS AU SABRE 1 . leur dissoute dans un liquide volatil, alcool ou essence, laquelle est amenée par de l'air sous pression et à travers un mince conduit jusqu'à une sorte de crayon métallique tenu à la main et d'où elle sort en un jet finement pulvérisé. C'est donc une manière de pinceau mobile dont on peut à volonté modérer l'action, comme la suspendre, de façon à laisser aux places choisies sur le support que l'on traite une trace colorée.

1 Communiqué par MM. Gauthier et Cle, Saint-Étienne. 
D'autre part, on a découpé les lignes du dessin et ses teintes dans une série de caches, pochoirs ou patrons, faits de carton ou de métal mince; on applique ces feuilles sur le tissu et il n'y a plus qu'à suivre les contours ajourés avec le jet de l'aérographe pour colorier. Une main adroite suffit à cela, sans qu'il soit besoin d'un bien long apprentissage ni de dispositions exceptionnelles. La production est facile, rapide, peu coûteuse par conséquent ; les frais de confection des caches sont bien moins élevés, presque constamment, que ceux de la gravure des planches ou des rouleaux d'impression. Et cependant ce genre de décoration, qui a eu, pour les tissus de soie, un succès notable et paraît devoir conserver une certaine place en répondant à certaines exigences commerciales, est-il vraiment appelé, comme on l'a pensé et dit, à remplacer un jour, même complètement, la belle impression à la main, sinon la peinture?

Il semble pourtant que de grands progrès sont encore à faire pour qu'il en soit ainsi. Il y aurait imprudence à répondre de ce que sera l'avenir pour une industrie comme l'impression, liée en somme à des progrès scientifiques de la chimie autant quà des perfectionnements mécaniques et où la voie reste ouverte à toute l’ingéniosité des chercheurs et à la subtilité des innovateurs; mais il est assez supposable, que pour longtemps encore, lorsque le temps et l'argent ne feront pas question, les œuvres du travail manuel, artistement et intelligemment guidé, resteront de beaucoup les plus recherchées.

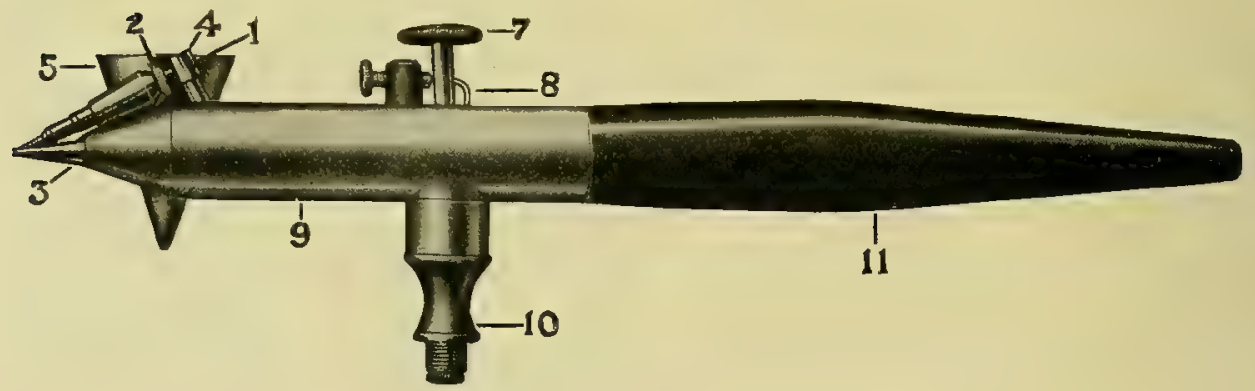

Pinceau d Aérographe pulvérisateur de couleurs.

(Cliché de la Société Franco-Américaine, Paris.) 


\section{RUBANS DE SOIE}

C'est une catégorie assez à part et très intéressante que celle des rubans de soie, parmi toutes les autres de la soierie proprement dite à laquelle elle se rattache par trop de points communs cepenđant pour n'être pas étudiée en même temps. Après tout, il n'y a là qu'une simple question de largeur; le ruban est une sorte de soierie en réduction ou en miniature; préparation et emploi de matières, jeux de fils ou armures, façonnage ou décor, tout ceci repose sur des principes qui sont les mêmes et des données analogues; 1e tissage seul diffère par certains points.

Cette fabrication qui s'exerça fort anciennement en divers pays, Hollande, Italie, Angleterre, Allemagne, Suisse, eut en France ses Maîtres Ribandiers, Tissotiers ou Passementiers (ces trois appellations étaient équivalentes), à Lyon, à Tours et à Paris, avant que de s'installer dans le Forez, à SaintChamond et à Saint-Étienne, pour prendre peu à peu dans cette dernière ville le considérable développement qui lui a donné une réputation mondiale.

Ribandiers, tissotiers, passementiers étaient formés en corporations qui monopolisaient non seulement les rubans mais aussi certains autres accessoires du vêtement et de la garniture, galons, tresses, passements, franges, etc., et ces associations subirent de fréquents changements d'attributions et remaniements de statuts.

Les seuls métiers employés pendant fort longtemps furent le métier à basses lisses pour les rubans unis et à hautes lisses pour ceux façonnès ou à dessins; 1'un et l'autre de ces métiers, passés maintenant à l'état de souvenirs historiques, dans lesquels la navette était maniée et passée à la main, ne permettaient de faire qu'une seule pièce de ruban à la fois, quelque minime qu'en fût la largeur.

L'idée d'un métier pouvant tisser à la fois plusieurs pièces de rubans, placés les uns à côté des autres, dut venir de bonne heure à l'esprit de plus d'un inventeur, mais il se passa toutefois pas mal de temps avant qu'il fut possible de surmonter les difficultés pratiques d'un pareil mécanisme, capable d'actionner plusieurs navettes en même temps et permettant à l'ouvrier tisseur d'en régler le mouvement à volonté.

Une des premières traces de métiers à plusieurs pièces est la mention que l'on a retrouvée de l'un d'eux, mis au jour en 1677 , et qui était l'ouvrage de maîtres passementiers parisiens; il ne produisit, du reste, que de mauvais tissus et, sans attendre d'autres perfectionnements, les autres maitres de la corporation, inquiets peut-ètre de voir surgir un outil à grande production, le firent saisir et briser par les soins du célèbre lieutenant général de la police, de La Reynie.

En Flandre espagnole, et probablement pour des motifs analogues, le Roi Très Catholique avait rendu une ordonnance défendant de monter des métiers de même nature. 
Chassé des Pays-Bas, le métier à plusieurs pièces fut importé en Suisse et ce fut à Zurich qu'il reçut la consécration définitive, attestée par le nom qu'il conserva depuis : métier à la zurichoise. Les fabricants de Bâle où l'on travaillait le ruban l'adoptèrent et alors commença pour Saint-Étienne une concurrence qui aurait ruiné l'industrie rubanière dans cette cité, si, peu à peu, avec le secours de la Royauté accordant privilèges et primes à cet effet, des métiers suisses n'avaient été importés et installés en France. Il y en ẹut au

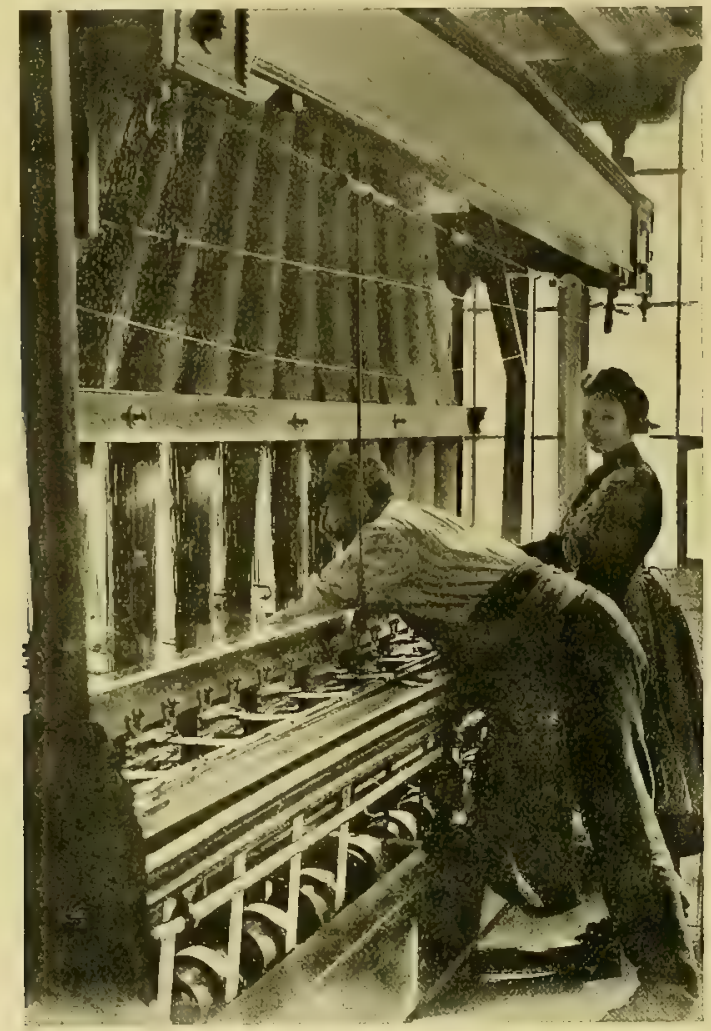

MÉtier de RUbans 1.

début à Marseille, à Nîmes, à Paris, où l'opposition des maîtres avait cessé, enfin à Saint-Chamond et à Saint-Étienne. Avec de grands sacrifices d'argent, malgré toutes sortes de risques et contre l'opposition des ouvriers du pays, manifestée par des grèves, on fit venir avec les métiers des ouvriers suisses experts à leur montage et à leur mancuvre. Si bien qu'à la veille de la Révolution on compte qu'il y avait au moins douze cents de ces métiers fonctionnant dans les deux villes foréziennes.

Des perfectionnements nombreux furent apportés depuis lors à ce métier

1 Cliche L. Merlat, Saint-Étienne. 
à la zurichoise, plus connu encore par la suite sous le nom de métier à la barre, ladite barre servant à l'ouvrier tisseur pour actionner tout le mécanisme du métier. Maintes innovations ingénieuses modifièrent les unes après les autres le battant, partie la plus importante du métier dans laquelle des organes délicats commandent le mouvement des navettes multiples.

Puis à ce métier qui ne pouvait fabriquer en premier lieu que des rubans unis, on s'efforça d'appliquer la mécanique Jacquard pour produire des rubans façonnés, ce qui demanda beaucoup d'essais, de tâtonnements et pas mal de temps.

Sous la Révolution, fut installé à Saint-Étienne le métier à fabriquer les rubans velours, à doubles pièces superposées et à rasoir automatique par les fabricants Thiollière, Duchamp et J.-B. David.

Enfin, depuis une vingtaine d'années, l'application du moteur électrique au métier à domicile remplaçant l'effort musculaire imprimé par l'ouvrier à la barre du métier, et aussi des installations en usine, ont doté la fabrication rubanière d'instruments de puissante production, trop puissante quelquefois en face de toutes les difficultés créées par les concurrences étrangères nombreuses et les revirements de la mode. Et celle-ci, en ses caprices imprévus, a bien des fois adopté, délaissé, repris, abandonné le ruban, comme tous les autres accessoires de la toilette, à partir de l'époque où les hommes en portaient encore davantage, si c'est possible, que les femmes, Mignons de HenriIII, Galants de la Fronde, Roués de la Régence, jusqu'aux élégances du XVIII ${ }^{\mathrm{e}}$ siècle où il fit souvent fureur dans le costume féminin.

On s'est évertué depuis longtemps à exécuter en rubans tous les genres ou à peu près de soieries, taffetas, satins, armures, velours, gazes, mélanges d'armures, rayés, filetés, quadrillés, barrés, façonnés à grands et petits dessins, moirés, gaufrés, plissés, imprimés sur chaîne ou directement, depuis la légère faveur et la minuscule comète jusqu'aux larges ceintures, en passant par une série nombreuse et graduée de numéros, dont chacun représente une taille respective. Et l'on a mis dans toutes ces fabrications une telle variété dans la recherche des jeux et des emplois de fils, dans le coloris et dans l'exécution habile des tissages les plus délicats et les plus ingénieusement compliqués qu'il importe de souligner le mérite sans conteste de ces créations.

Il y a de plus encore, des rubans de soie mélangée avec la schappe, la laine, le coton, le fil, la soie artificielle; mais il n'y a pas à proprement parler de rubans teints en pièces, en raison des difficultés toutes spéciales que présentent la teinture et l'apprêt, avec les largeurs si réduites en cause. Pour des rubans donnant l'apparence du crèpe ou de la mousseline de soie, force a donc été de tourner la difficulté en usant, par exemple, de soies moulinées spécialement d'une façon très tordue, après leur teinture, etc.

Une autre solution approchée du problème a été de fabriquer, en s inspirant du tissage des pièces jumelles, des mousselines ou des crêpons dans les largeurs habituelles, mais toutes divisées en bandes ou rubans, ayant chacun leurs lisières et séparés seulement par un très petit espace, dit jour de gaz̃ $e$, parce qu'il se trouve limité et déterminé par deux fils de tour qui l'encadrent; après teinture et apprèt, on découpe ces rubans au moyen de lames tranchantes qui glissent entre les fils de tour; ceux-ci retiennent les lisières suffisamment pour empêcher le tissu de s'effiler. 
Le même artifice s'emploie couramment et largement pour des soieries plus fortes comme des satins et d'autres armures le plus souvent tramées avec du coton. Ces rubans découpés qui ont pour eux la commodité et la rapidité d'une fabrication ne réclamant pas un outillage particulier et la facilité de teinture du tissu après coup dans la teinte qui se trouve demandée, concurrencent, on l'imagine, avec un sérieux avantage de prix, les rubans véritables tissés teints, s'ils n'en ont pas toujours la belle apparence, la qualité et le fini.

Les tresses, lacets, ganses, lézardes, franges, chenilles, de soie ou de matières analogues sont autant de spécialités dont la fabrication s'exécute aujourd'hui au moyen d'outillages mécaniques très perfectionnés et particuliers à chacune.

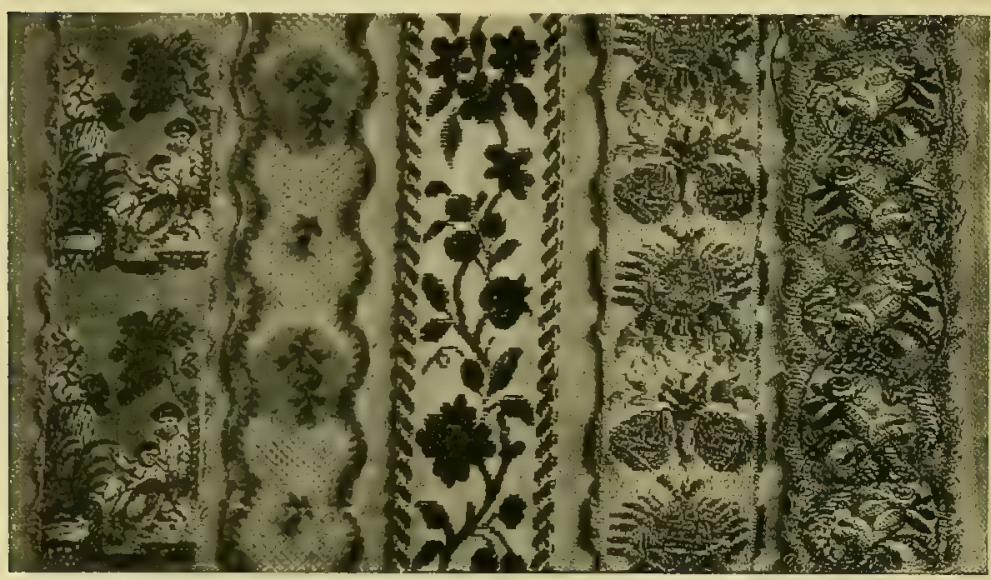

Rubans d'ancienne fabrication. (Musée des Arts décoratifs, Paris.) 


\section{SPÉCIALITÉS ET CURIOSITÉS}

\section{DU TISSAGE DE LA SOIE ET DE SON DÉCOR}

Par spécialités nous entendons ici certains genres de tissus de soie fabriqués en vue d'emplois bien définis et qui se réclament de caractères assez constants sous divers rapports.

Telles sont par exemple:

- Les soieries avec lesquelles on confectionne des cravates, ou soieries dites pour col, encore que cette consommation se soit étendue depuis plusieurs années, d'après une tendance très éclectique, à l'utilisation de tissus fort divers : unis, fantaisies, façonnés, imprimés, et le plus souvent établis pour des emplois tout autres.

- Les soieries pour doublures, unies et façonnées; dans celles-ci le lot des tissus mélangés et teints en pièces occupe assez naturellement une place prépondérante, et cependant on a vu et on voit parfois employer, pour doubler des fourrures de prix ou plus simplement même des étoffes assez quelconques, de belles soieries, satins, damas, et des lamés or et argent.

- Les soieries employées à confectionner les parapluies; l'emploi universel de cet accessoire a fait de cette spécialité une branche industrielle et commerciale très importante; ces tissus qui ne comprennent guère d'autres armures que le taffetas et le sergé, exigent avec des soies de premier ordre une fabrication très soignée et qui, pratiquée autrefois à la main, est aujourd'hui exclusivement mécanique, pour obtenir la résistance en même temps que la régularité de l'ètoffe; celle-ci est très réduite, c'est-à-dire que les fils en sont fort serrés, et en même temps doit rester légère et souple; on augmente notablement cette souplesse et la couverture du tissu par l'opération que nous avons déjà signalée du polissage.

Une étoffe, teinte en pièce, chaîne soie et trame laine, a conquis depuis trente ans environ une place très importante dans la confection du parapluie, sous le nom de Silésienne.

Les ombrelles demandent, quand on ne se borne pas à utiliser pour leur confection des soieries quelconques de robes, un tissage particulier, comportant généralement des bordures qui servent d'encadrement et une largeur convenable pour éviter les pertes lors du montage et de la confection de ces objets.

Il faudrait encore mentionner : les soieries qui conviennent à la gainerie, celles comme certains taffetas, satins, velours, que l'on utilise dans la fabrication de fleurs artificielles, des soieries pour corsets, pour voitures, pour drapeaux, et aussi pour ballons donnant des enveloppes légères qu'un vernis vient ensuite imperméabiliser, etc., etc. Mais une spécialité bien typique à ne pas oublier est celle des gazes de soie à bluter; employées à faire des tamis très fins, destinés au blutage des farines dans les minoteries, c'est-à- 
dire pour séparer après mouture la farine du son et des corps étrangers, ces gazes sont faites de soie écrue ou grège, filée tout spécialement pour être régulière et tenace, et sont tissées ensuite dans la largeur habituelle d'un mètre, soit en gaze droite (fils croisés en taffetas), soit en gaze à fil de tour; les unes ou les autres comportent des réductions ou degrés de serrage des fils, dont quelques-unes sont tout à fait fines et comportent de 40 à 50 et jusqu'à go fils dans I centimètre et dans les deux sens, celui de la chaîne et celui de la trame; on apprête légèrement ces gazes en écru et on les emploie sans autre préparation.

Certaines étoffes de soie, pures ou mélangées, fabriquées en vue de l'exportation en Orient, aux Indes, en Chine, sur des données à peu près constantes comme composition et comme décor, peuvent être rangées dans une classe que l'on est en droit encore de considérer comme l'une de ces spécialités de fabrication qu'un examen minutieux révèlerait nombreuses.

A la rubrique des curiosités du tissage de la soie, il faut en premier lieu mentionner les mélanges que l'on a faits de cette matière textile avec d'autres substances assez imprévues, en obtenant des combinaisons de succès éphémère et bien de pure curiosité pour la plupart. Exemple: les taffetas tramés avec des cheveux, des crins, des plumes, de légers filaments de bois, de paille et aussi de verre.

Cette dernière association, fort paradoxale à première vue, de deux produits d'espèce bien différente, et sur laquelle on a fondé à son apparition, vers 1839 , plus d'espoir qu'elle n'en méritait peut-être, était réalisée en employant des filaments de verre filé obtenus si fins, qu'il fallait en prendre quarante ou cinquante à la fois pour passer un seul coup de trame et non point avec une navette, mais avec le secours d'un outil ou crochet spécial. On obtenait de la sorte des étoffes d'une souplesse toute relative évidemment, mais auxquelles le brillant du verre et la teinte qu'on pouvait lui donner préalablement, communiquait un éclat assez remarquable; cette opposition du verre avec la soie était heureuse pour des tentures, des rideaux, des ornements d'église, et toutes étoffes exposées le moins possible à des froissements et des déplacements réitérés.

Pour le Retour des Cendres, en 1840, la Chapelle des Invalides fut entièrement tendue d'une étoffe faite d'après ce curieux procédé de fabrication; la chaîne était de soie violette, couleur « pensée » et la trame de verre filé couleur dor, jouant le métal ; le tissu était façonné de dessins de feuillages, d'aigles et d'N, dans la bordure. On rapporte qu'une partie de cette même étoffe, ayant servi de drap mortuaire, fut fragmentée et distribuée en souvenir aux personnages de marque présents aux Tuileries lors de cette mémorable cérémonie; certaines parties de cette tenture sont encore conservées au Mobilier National.

Aux chapitres des soieries unies nous avons parlé déjà de plusieurs problèmes de tissage élégamment résolus, comme pour ces tissus de soie fabriqués sans envers ou en double face, et encore en double étoffe, par deux pièces exactement superposées; une curiosité dans ce dernier cas est de faire que les deux pièces se trouvent réunies par les lisières, si bien que le tissu formé se trouve être une sorte de tube ou de tuyau sans couture. A ce même ordre de difficultés pratiques vaincues par d'ingénieux systèmes, on 
peut rattacher la fabrication de tissus présentant des plis tissés, ce qui produit l'aspect d'une étoffe sur laquelle on aurait cousu de place en place régulièrement et tout de son long de petits rubans attachés par une seule de leurs lisières et flottant sur l'autre côté. Il en est de même des tissus circulaires, inventés et brevetés par le sieur Grégoire, en r 800, sous le nom de Tournoises; la trame sy disposait pour ainsi dire en éventail, constamment plus serrée sur une lisière que sur l'autre; ils ne pouvaient guère convenir qu'à des usages restreints et restèrent pratiquement sans emplois.

Les ressources très variées, comme nous l'avons vu, dont on dispose pour décorer les soieries devaient aussi fournir aux chercheurs d'inédit et à ceux que n'arrêtent pas les difficultés, les moyens de réaliser des applications curieuses des jeux de fils et dont on a tiré parti de tout temps ou à peu près pour retracer sur les étoffes autre chose que des dessins inspirés de l'imitation de la fleur, de l'ornement, etc. Une, entre autres, assez remarquable, est celle des portraits et des tableaux tissés en soie.

Ce ne fut en somme, qu'une manière plus poussée, plus détaillée, grâce à des organisations de métiers plus perfectionnées, de rendre la figure, bien souvent utilisée dans le décor de nombreuses

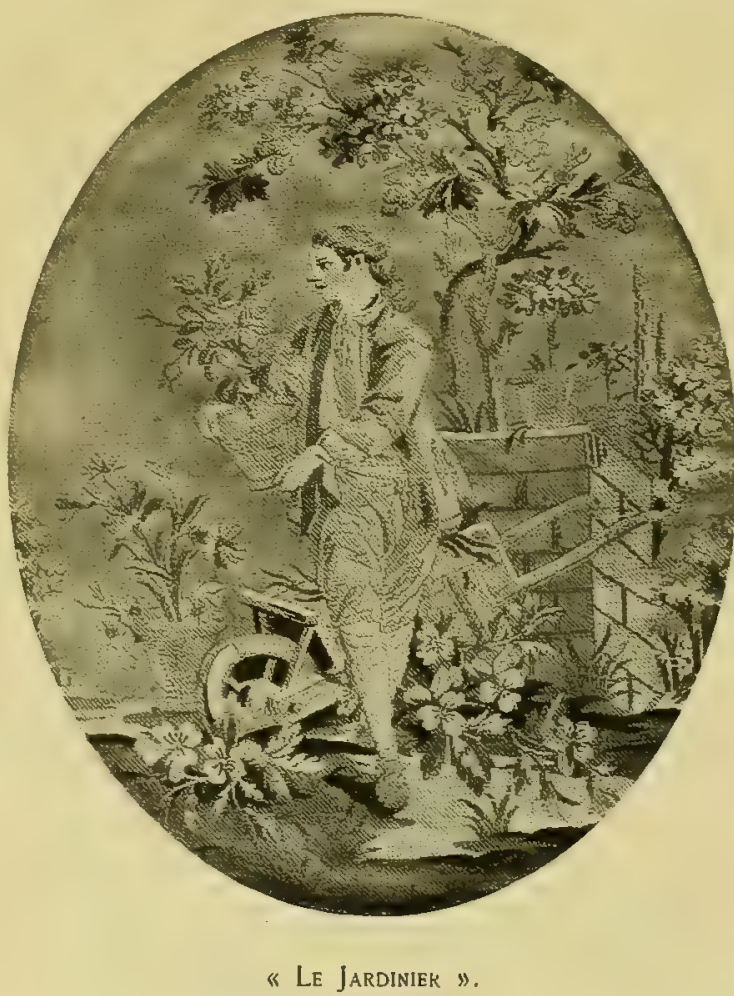

(Soierie pour sièges. Composition de Ph. de Lasalle.) soies anciennes et de façon si intéressante et artistique, en particulier, comme nous l'avons rappelé, dans les broderies. A Lucques, et à Sienne déjà, on avait tissé en soie et en soie mêlée d'or maintes sortes de petits tableaux du genre religieux, comme des Annonciations, avec une habileté remarquable pour l'époque, mais c'est à partir de la fin du Xvirl ${ }^{\text {e }}$ siècle et surtout après l'invention de Jacquard, que l'on voit se préciser ces tentatives.

Philippe de Lassalle, parmi les grandes compositions dans lesquelles il fait jouer les soies de toutes couleurs, la chenille, etc., et illustre leur décor non seulement de fleurs, de feuillages, d'attributs, mais d'oiseaux, paons, faisans, perdrix, admirablement retracés et nuancés, ne craint pas de s’attaquer au problème et, dans un médaillon encadré d'un arrangement de fleurs qui nous a été conservé et se trouve au Musée historique des tissus de Lyon, dessine en touches simples, mais habiles, marquées par des soies brochées de 
plusieurs tons camaïeux, le portrait en profil de médaille de la grande Catherine de Russie. Il avait fait, paraît-il, en soie également, les portraits de Louis XV et du comte de Provence, et il ne paraît pas inutile de rappeler qu'on lui doit ces petites compositions charmantes exécutées à l'usage de sièges, telles que le "Jardinier » et la "Jardinière », qui sont restées comme des modèles de petits tableaux de ce genre, par la simplicité et la justesse des effets produits par le travail des soies de couleurs, souligné de légères retouches de noir bien placées.

L'art du portrait tissé en soie s'est beaucoup développé au Xrx ${ }^{\circ}$ siècle ; il s'est formé en premier lieu toute une iconogra phie napoléonienne de cette sorte. Un écran retraçant en velours sur fond de satin les traits du Premier Consul et un autre dont le décor est en velours vert sur fond côtelé blanc et porte en son milieu l'inscription : "Il nous a donné la paix », avec, au bas, la mention tissée également: "Fait en présence du Premier Consul, à Lyon, le 26 nivôse an $\mathrm{X} »$, rappellent la visite mémorable que fit Bonaparte à la cité de la soie et lintérêt qu'il portait au relèvement de son industrie éprouvée par la Révolution; c'est alors qu'il se rendit dans les principaux ateliers et ne dédaigna pas, dit-on, de prendre place devant un métier et de passer quelques coups de navette. (E. Dumonthier, Etoffes napoléoniennes.)

Mais un portrait obtenu en velours par le tissage seul n'est à la vérité quine simple silhouette se découpant sans beaucoup de détails sur le tissu de fond où elle se trouve comme plaquée; on ne peut trop la modeler que par de simples retouches de frist sur le coupé par exemple; ainsi fut rendu le profil typique du Petit Caporal en des images de petites dimensions qui ont été populaires et assez répandues.

Pour plus de précision et d'expression, il a fallu recourir aux jeux complexes des effets ou points de trames, surtout en les ordonnant par une mise en carte très soignée et en recherchant la plus grande finesse possible d'exécution par l'arrangement du métier. On peut user dans ce but de trames qui sont brochées ou surajoutées au tissu de fond, un peu comme une broderie, et c'est ainsi que fut exécuté un portrait de l'empereur Napoléon I ${ }^{\text {er }}$, par Gantillon, habile fabricant lyonnais; il avait fait breveter, en i 832, le procédé spécial de broché qui lui permettait d'obtenir, par l'emploi de plusieurs trames teintées en camaïeu dégradé et la juxtaposition de leurs points très fins, une grande exactitude; dans le portrait en question qui est sur un fond de satin de couleur vive, les lignes du visage et tous les détails, même assez menus, se distinguent fidèlement rendus; il en est de même dans les autres portraits et les compositions exécutées par cet inventeur, genres de tableaux tissés tels que « les environs de Naples », et « la vue du lac de Côme »; il s'était flatté de faire adopter l'emploi de ces étoffes brochées, comme de sortes de tapisseries de soie, convenant à tous les usages de la décoration.

Un autre procédé pour obtenir des portraits et des tableaux tissés par lequel on parvient à l'imitation parfaite de la gravure et de l'estampe est celui qui consiste à prendre pour fond un satin blanc fait par une chaîne convenablement fournie, et en tramant au contraire avec de la soie noire, celle-ci se trouvant dans le fond, selon notre description du satin, complétement dissimulée; dans les parties du dessin, elle forme, ensuite d'une dispo- 
sition à la Jacquard assez complexe, des points ou flottés tantôt fins et même très fins, tantôt, au contraire, suffisament accentués; et le dessin, portrait ou tableau, est rendu par : des noirs francs qui sont des flottés de trame, des blancs obtenus par les fils de la chaîne; et, pour les tons intermédiaires, par quantité d'armures et de combinaisons de la chaîne avec la trame, réglées

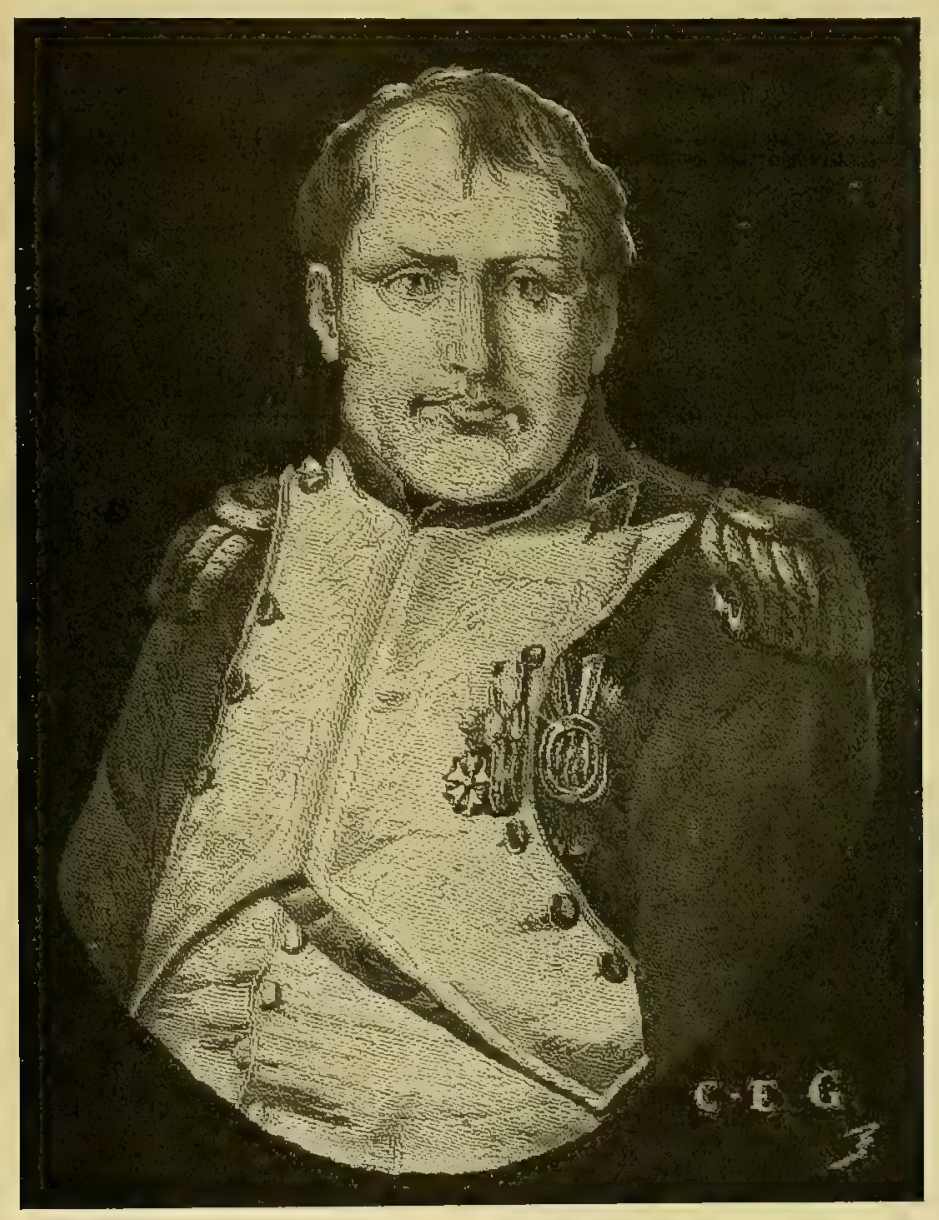

Portrait tissé de Napoléon I $^{\mathrm{er}}$, par Ganthllon.

par la carte, qui fournissent tous les gris dégradés qui sont nécessaires. On reste étonné đu modelé et du fini auxquels on peut parvenir par ces moyens de tissage qui ne sont pas évidemment de pratique tout à fait courante, en raison des soins qu'il faut apporter à l'organisation des métiers, du temps qu'il faut passer à la préparation du dessin, à la mise en carte, etc., et réclament une collaboration étroite et obligée entre le dessinateur, le fabricant et le tisseur.

D'après cette méthode ont été exécutées des cuvres assez nombreuses, 
parmi lesquelles il faut relever en premier lieu le portrait de Jacquard ${ }^{1}$, fait, en 1839 , par Didier-Petit, daprès le tableau de Bonnefond, peintre 1yonnais; ce fut un hommage tout naturel rendu à l'inventeur génial de la célèbre mécanique, tout comme plus tard, en 1854 , les portraits de Philippe de Lassalle, de Bony, de Bergeon, par Reybaud, fixèrent dans la soie les traits

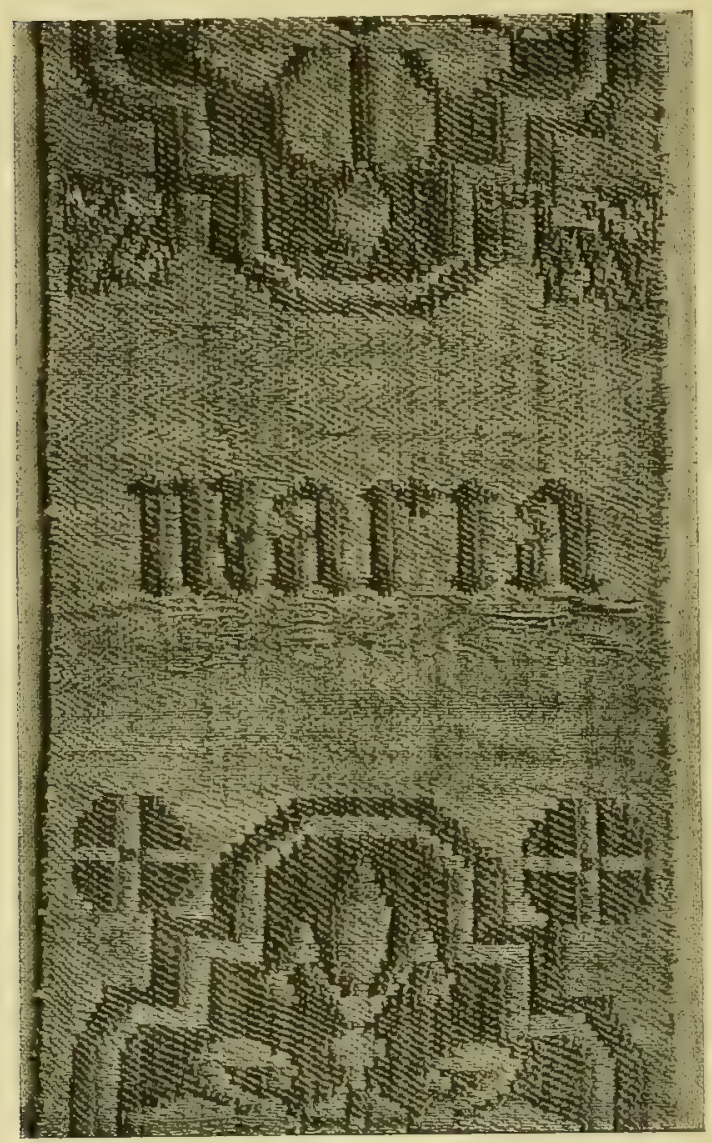

TISSU dIT dE COLOGNE ?

de ces remarquables artistes.

Citons encore divers portraits ou tableaux consacrés à des personnages de marque : le portrait de Louis-Philippe, celui de Napoléon III avec la famille impériale; le duc d'Aumale visitant latelier de Carquillat, à la Croix-Rousse, tableau tissé d'après une composition de Bonnefond, et qui retrace très curieusement les détails de cette visite d'un prince royal chez le canut qui devint célèbre par son habileté et sa spécialisation dans le tissage justement de ces genres de travaux, et devint le portraitiste des Chefs d'Etats.

Et encore : un remarquable portrait de Washington, par Mathevon et Bouvard, un tableau tissé d'après la Vierge de Saint-Jean, par Lamy et Giraud, enfin quantité d'autres œuvres du même genre, exécutées par divers fabricants, soit à propos d'expositions universelles, soit pour servir de sortes de marques à leurs maisons, etc. L'industrie stéphanoise, également, a fabriqué en ces dernières an. nées des rubans reproduisant, d'après la mème technique, nombre de portraits ou de reproductions de tableaux.

Puisqu'il est possible, avec des fils de soie convenablement commandés et manœuvrés, d'arriver à tracer sur le tissu de soie les lignes d'un portrait, à plus forte raison est-on en mesure d'y former, par les mêmes moyens, des lettres, décoration qui n'exige pas toujours une grande finesse de détail. Aussi voit-on fort anciennement des inscriptions figurer sur des étoffes; à l'époque médiévale, entre autres, ce fut un usage fréquent d'inscrire des

1 Voir la reproduction de ce portrait donnée page 95.

2 Musée des Arts décoratifs, Paris. 
noms, des dates, des versets de psaumes, sur des étoffes désignées, il y a tout lieu de le croire, par le terme de «litteratce» que l'on relève dans les textes documentaires de l'époque, toutes inscriptions tissées la plupart du temps de la façon que l'on remarque sur les tissus dits de Cologne, conservés dans certains musées. Il en devait être de même, c'est supposable tout au moins, pour cette ceinture de soie mentionnée dans l'inventaire de Charles VI, « ou estait escripte l'évangile de Saint Jehan »; et c'est une inscription tissée qui fixe fort heureusement, du reste, la date (528 de l'hégire) du fameux manteau impérial de Nüremberg, de travail palermitain, qui est à Vienne maintenant.

Il y a loin de ces inscriptions au travail remarquable d'imitation parfaite de la typographie exécuté en I 827 par Maisiat, professeur de tissage à Lyon, avec le secours de la mécanique Jacquard et avec le montage du métier à tringles, dont il avait eu l'idée et permettait la grande finesse déjà signalée plus haut pour les portraits.

Maisiat reproduisit donc sur un fond de gros de Tours chaine blanche et avec une trame noire, d'abord le testament de Louis XVI, puis la dernière lettre de Marie-Antoinette; ces deux compositions en format grand in-folio, texte sur deux colonnes avec marges, en caractères romains, et comportant des encadrements d'ornements avec médaillons et portraits, représentent de vrais chefs-d'œuvre de patience et d'habileté pour la mise en carte et de soins pour le tissage; tout ceci réalisé de telle sorte que ces épreuves ayant été présentées lors de leur achèvement au célèbre typographe Firmin Didot, «il fut saisi d'étonnement» et ne put croire que ce qu'on lui montrait était le résultat d'un tissage.

Une cuvre contemporaine du mème genre et d'un grand mérite est le Livre d'Heures, entièrement tissé, que l'on doit à M. Henry, fabricant lyonnais, continuateur des meilleures traditions de goût et de savoir de cette profession; ce texte relevé de lettres ornées, d'enjolivures, et d'encadrements d'après des manuscrits anciens, représente un labeur considérable.

N'oublions de mentionner parmi les imitations tissées d'estampes, les très curieux paysages exécutés par Furnion en velours façonné, par mélanges et dégradations de tons blancs avec d'autres noirs ou bistres; ils témoignent d'une grande dextérité de composition et de fabrication.

D'autres sortes de très curieux velours avaient, bien avant ceux-ci, uni les procédés du tissage à ceux du chinage et à la peinture dans certaines productions restées limitées. Citons : les velours chinés de Richard, du nom du maître chineur réputé auquel leur travail est attribué; tel, par exemple, ce profil de Bonaparte se détachant sur un fond garni d'emblèmes maçonniques, dont l'intérêt n'est que de curiosité rétrospective du reste:

Les velours Grégoire sont tout autre chose et procèdent d'une combinaison remarquable de la peinture exécutée sur les fils de soie avant le tissage définitif, avec ce tissage lui-même; ce sont des tableaux de petites proportions, fleurs, sujets, portraits, dont la valeur par le mérite de la peinture et le charme des colorations obtenues, fondues et adoucies qu'elles sont par le travail du velours, est celle de véritables cuvres d’art. Grégoire était né à Aix-enProvence, en 175 I il vécut à Paris, oủ il mourut en 1846 ; il ne révéla jamais le véritable secret des manipulations délicates qu'il avait inventées pour 
obtenir ces velours qui restèrent, contrairement à son attente, en dehors du domaine pratique. Imités à plusieurs reprises, les velours Grégoire n'ont pas été égalés.

Antoine Vauchelet, établi à Parisvers 18 io et qui décorait des velours par l'impression, combinée la plupart du temps avec la peinture à la main, pour des écrans, des objets d'ameublement, appliqua ses procédés à des velours de

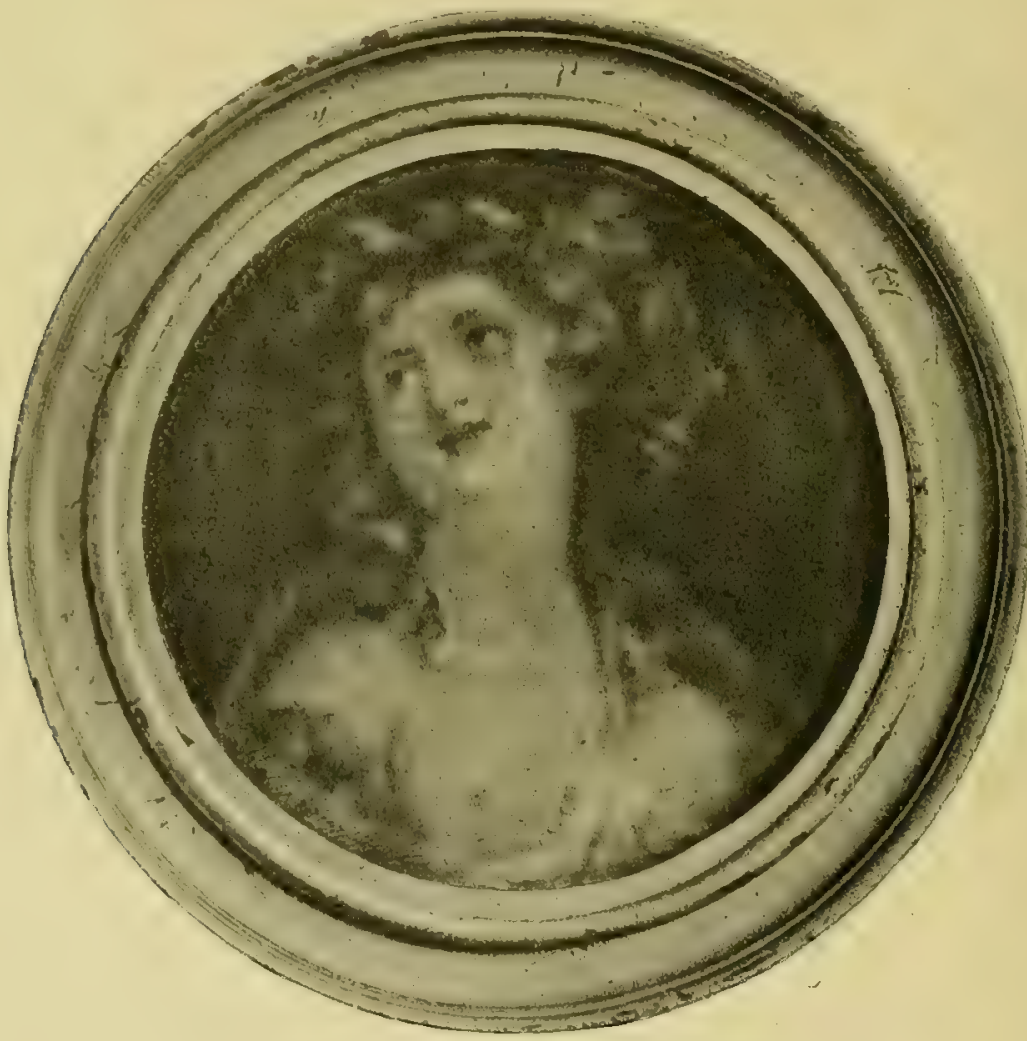

Velours GréGotre. (Musée de Marseille.)

soie dont il existe, au Mobilier National, de très intéressants spécimens. Associé avec un nommé Neuville, il fabriqua deux importantes tentures de meubles entièrement ou presque peintes à la main, par des artistes connus; l'un de ces meubles, avec des vues de Rome, fut destiné au palais du Luxembourg pour le salon du Roi de Rome, l'autre, avec des vues de Paris, fut envoyé à Naples. (E. Dumonthier, Etoffes napoléoniennes.)

Le taffetas, le satin, formant à tout prendre un support uni et lisse assez analogue au papier, on a eu de bonne heure l'idée d'utiliser ces tissus pour des impressions typographiques de luxe. Une liste assez importante a pu être établie de livres imprimés sur soie, taffetas ou satin blanc, qui sont des curiosités de bibliothèques. Au temps dè Boileau, l'usage était fréquent 
d'imprimer sur soie les thèses des nouveaux docteurs en Sorbonne et lui-même y fait allusion dans les vers connus de la satire $X$ :

Peindrais-je son jupon bigarré de satin Qu'ensemble composaient trois thèses de latin,

De même, et souvent, on a tiré des estampes sur soie; Dagoty avait fait de curieux tirages de gravures en couleurs, mais sur velours de coton; sous le Directoire et l'Empire on fit à la planche d'acier ou de cuivre finement gravée, des tirages sur satin, de petits sujets dans le goût mythologique, dont les dessins rappellent la manière de Prud'hon ou de Clodion, en médaillons, encadrements ou frises destinés à des ornements de vêtements à la grecque et à des bordures d'ameublement; l'exécution en est particulièrement soignée et intéressante.

Gravures, dessins à la plume, phototypies se rendent également bien tirées en noir, en bistre, etc., sur fond de satin ou de taffetas blanc. La chalcographie du Louvre, par exemple, obtient de cette façon des épreuves recherchées par les amateurs.

Enfin, il existe de la soie à usage photographique, c’est-à-dire du taffetas à grain fin qui, sensibilisé à l'avance, puis impressionné à la lumière au châssis par contact avec un négatif sur verre et manipulé comme à l'habitude ou avec quelques soins plus particuliers peut-être, donne des épreuves appréciées pour leur douceur, leur matité et leur souplesse.

Il y a encore des soies électriques, ou mieux des tissus dans lesquels les propriétés de la soie, matière animale éminemment apte à dégager de l'électricité développée par le frottement, se trouvent très augmentées par un traitement spécial, au point que, sous le frottement de la main sèche, ils dégagent desétincelles fort sensibles; c'est, pour le moment, une curiosité de labora toire qui pourrait, peut-être, un jour rencontrer des applications beaucoup plus étendues.

On pourrait sans doute grossir sensiblement, plus que nous ne l'aurons fait dans ce dernier chapitre et les précédents, la liste des applications curieuses ou spéciales qui peuvent exister dans les arts et dans l'industrie, comme de toutes celles quel'on relève un peu partout, d'une matière textile aussi répandue que la soie l'est aujourd'hui; c'est ainsi qu'il faudrait ne pas oublier le rôle important de la soie employée comme fil à coudre et à broder, qu'il conviendrait de mentionner les tapisseries et les tapis de soie ${ }^{1}$, etc, etc., mais ne faut-il pas aussi savoir se borner?

Ne suffit-il pas, au reste, de tourner les yeux avec quelque attention un peu partout pour se convaincre de cette universalité de la soie que nous nous attachions à relever dès l'avant-propos et qui se manifeste de cent façons et dans mille détails qui restent le plus souvent inaperçus ou négligès ?

Sans doute une loi très générale fait que pour tous, ou à peu près, tous les

1 Exemple : ces tapis d'Orient, et ces curieux tissus dits : gobelins chinois et japonais. 
produits qu'un industrieux travail de fabrique et de perfectionnement transforme et façonne au cours de nombreuses opérations successives et apporte tout prêts, tout finis à l'usage, on ne retienne plus, on ne voit plus que la commodité, la facilité de l'usage agréable ou pratique, sans trop songer à tous les travaux préparatoires de la manufacture.

Cependant, elle est, cette progression, particulièrement lente pour la soie, retardée qu'elle se trouve par mille soins dèlicats, depuis l'instant où la chenille a filé cette soie pour se construire une provisoire retraite, jusqu'au moment où, transformée en étoffe, elle est offerte à tous les besoins venus pour la grosse part d'une préoccupation d'élégance ou bien inspirés par le souci du simple confort.

Ainsi, les Arts multiples de la soie forment, on pourrait dire, comme les étapes de cette marche qui comportent ses accidents et ses subtilités.

C'est une simple esquisse que nous avons tenté de tracer de tout cet ensemble imposant, plutôt que de prètendre aux descriptions détaillées, minutieuses et définitives; puisse-t-elle aider cependant, à se représenter mieux et plus équitablement qu'il n'est très commun, l'intéressante diversité, le caractère nettement artistique, comme aussi la considérable importance d'une industrie placée depuis longtemp.s déjà et pour longtemps encore, il faut le souhaiter, au premier rang des richesses françaises.

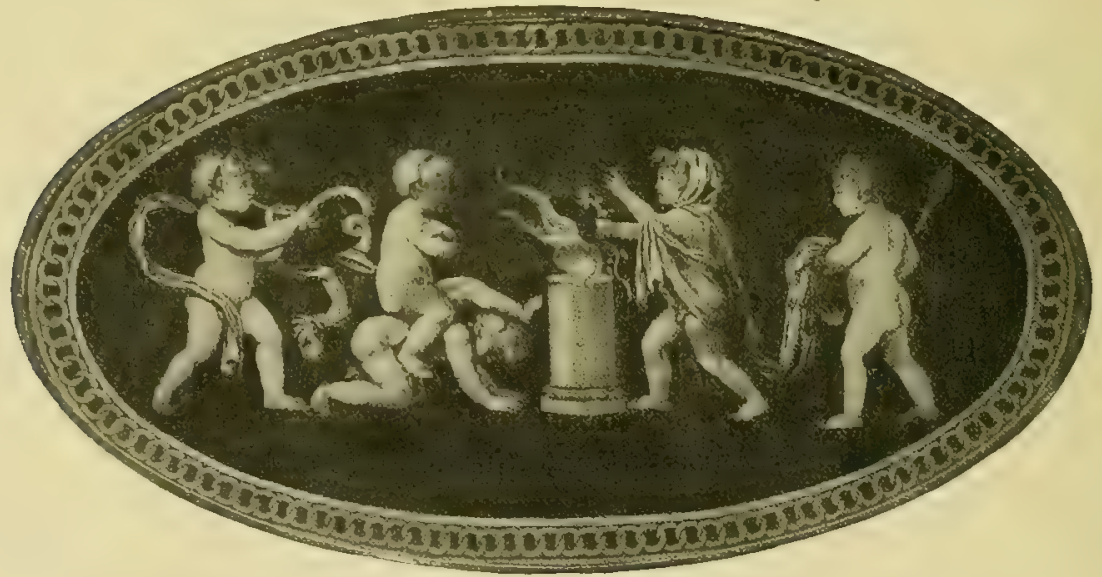

Sujet tiRE a la façon de L'Estampe, sur satin. (Époque Directoire ou Premier Empire.) 


\section{E R R A T A}

page $17,13^{\mathrm{e}}$ ligne : au lieu de $: .$. le ròle qu'auraient à jouer ces fils dans cette étoffe... lire :... le rôle qu'auront à jouer ces fils dans ces étoffes...

page 39, avant-dernière ligne, au lieu de ... c'est le prix et des bases de comparaison permettant...

lire .... c'est le prix et l'on y parvient trés bien cependant à l'aide de bases de comparaison permettant...

page $4^{2}$, dernier alinéa, au lieu de .... ne ferait-on pas remonter cet usage plus haut... live $: .$. ne ferait-on remonter cet usage pas plus haut...

page $58,4^{e}$ alinéa, au lieu de... et comme je l'ai dit...

lire .... et comme l'on dit...

page $67,3^{\mathrm{e}}$ alinéa, au lieu de $\ldots$... présentent avec l'aspect crêpé...

lire... présentent avec un certain déplacement l'aspect crêpé..."

page $7 \mathrm{I}, 2^{\mathrm{e}}$ alinéa, au lieu de $\therefore$... il serait nécessaire de faire...

lire... il soit nécessaire de faire...

page 87 , avant-dernière ligne, au lieu de .... comporte suivant l'entente...

lire :... comporte suivant son entente...

page $126,3^{\text {e }}$ alinéa, au lieu de... taffetas au gros de Tours...

lire :... taffetas ou gros de Tours. 



\section{TABLE ANALYTIQUE DES MATIËRES}

aérographe aiguilles de la mécanique Jacquard $\quad 9 \mathrm{I}$ apprêt . armures

- simples dérivées

artificielle (soie)

avivage

Badjer (moire de).

ballons (soieries pour)

barrées (soieries)

battage

battant.

- brocheur.

bengaline. brodeur

145

28
Bony . . . . . . . . . 128

brillantine . . . . . . . . . IIO

brocart . . . . . . . . . . I II

brocatelle. . . . . . . . . IOI

brochées (soieries) . . . . . . 105

- (soieries) de Gantillon. . . I5t

brochés semés (dessins) . . . . 107

- suivis (dessins) . . . . . 107

broché crocheté . . . . . . 109

broderies sur soie à la main . . . . I29

- sur soie au crochet . . . . I29

cannelé . . . . . . . . 53

cannette . . . . . . . . . 29

cannetille: . . . . . . . . . I24

cantre. . . . . . . . . 25, II6

Carquillat (atelier de) . . . . . ${ }_{5} 6$

Casanova (Essai d'impression sur soie par) .

cartons de la mécanique Jacquard

chaîne (fils de).

chenille

chevron

chinées (soieries). . . . . . . I.jt

cocon . . . . . . . . 12

Cologne (tissus dits de). conditionnement de la soie. . . . . I6

cordonnet. . . . . . . . . . . IT

corsets (soieries pour) . . . . . $\mathrm{r}_{51} \mathrm{I}$

crapaud . . . . . . . . . $3^{\mathrm{I}}$

cravate ou col (soieries pour). . . . I5I

crèpage . . . . . . . . 36, 69

crêpe (fil). . . . . . . . . . I7

- (tissu). . . . . . . 67

- anglais . . . . . . . 67

- lisse.. . . . . . . 67

— crèpé. . . . . . . 67

- de Chine. . . . . . . . . 68

crêpon mercerisé . . . . . . 68

crochets de la mécanique . . . . 9

cuite . . . . . . . . . 20

cylindrage . . . . . . . . . 31

damas . . . . . . . . . . . . 99

- caphart. . . . . . . . . IOI

dauphine . . . . . . . . . II3

décomposition des tissus de soie . . 40

décreusage . . . . . . . 20

dents (du peigne). . . . . . . 30

dernière lettre de Marie-Antoinctte

(Maisiat) . . .. . . . . I57

dessins contresemplés, sautés, à rama-

ges, etc. . . . . . . . 87

dévidage. . . . . . . . . 23

dichrolques (soies). . . . . . . . 22

disposition (de rayures). . . . . . 76

double-chaine (soierie) . . . . . . II3

— teinte (soies). . . . . . . 22

doublures (soieries pour) . . . . I5I

drapeaux (soieries pour) . . . . I5I

draps d'or . . . . . . . . I2I

droguet . . . . . . . . . III

échantillonnage. . . . . . . . . 39

écossaises (soieries) . . . . . . . 79

électrique soie). . . . . . . . I59

embuvage. . . . . . . . . . 25,77

éolienne . . . . . . . . 16

espolins . . . . . . . . . . 107

estampes tirées sur soie. . . . . I59 
façonnées (soieriesi . . . 39, 80 I 26 faille . . . . . . . . . 42

- française.

fantaisie (soieries).

fer (de velours).

filature.

filé (d'or).

fils manquants

flambage

flammees (soieries)

fleurs artificiolles soicries pour'.

florence

foulards

gaineric (soierics pour'

gaufrage

gaze à bluter

- it fils de tour

- façonnée.

- droite.

glacé (taffetas) .

gravure des planches d'impression. grige (soic)

grenadine fil

grillage

grille

gros grain

gros de Tours

groupure.

haitienne

I I I

impression ad hoc

- au rouleau

- directe

- par enlevage

- par application.

- par rongeant

- sur chaîne

jcté (du dessin)

Jacquard .

lame (d"or)

lamés (tissus)

lampas.

lancé

lat

levantine

ligature

lisage

lisses

litteratoe (étoffes).

livres d'heures tíssé, M. Henry louisine
I $26, \quad$ I $\$ 5$

I 37

I 37

138

I 38

$\mathrm{I}_{3} 8$

I 42

85

123

I 24

$\mathrm{IO}_{3}$

IOI

IO2

48

IO2

90

28

I57

I 57

$15^{8}$ mailles. : . • • . . . . . 29

main (impression à la) . . . . . 137

magnanerie . . . . . . . . . . $I_{3}$

marabout (fil) . . . . . . . . . I7

marceline. . . . . . . . . . . 46

marches . . . . . . . . . . 29

mélangées (soieries) . . . . . . . 40

mercerisage . . . . .. . . . . . 69

mitier à la Falconne. . . . . . . 93

- à la marche. . . . . . . . II2

- à la petite tire. . . . . . II2

- à la grande tire . . . . . . 92

- à la tire . . . . . . . . . 92

- à broder de Saint-Gall . . . I 30

- de Vaucanson . . . . . . 9.3

- de rubans à basses lisses . . . I 47

- - à hautes lisses . . . ${ }_{1} 17$

métier de rubans, à la barre. . . . I49

mexicaine. . . . . . . . . . III

mézeline . . . . . . . . . . . IO2

milanaise (fil) . . . , . . . . . I7

mise en carte . . . . . . . . . 89

moirage . . . . . . . . . 36

moire . . . . . . . . . 44

- (soies pour). . . . . . . . 22

- antique. . . . . . . 44

- ronde ou française. . . . . 45

montage (des métiers) . . . . . 96

moulinage . . . . . . . . I7

mousseline de soic . . . . . . 63

navette . . . . . . . . 29

ombrelles (soieries pour) . . . ". . I5 I

ondé (fil). . . . . . . . . . . Iy

or filé . . . . . . . . . . 124

- frisé . . . . . . . . I24

- trait. . . . . . . . . . $\mathrm{T} 23$

organsin . . . . . . . . . I7

Orient (soierie pour 1') . . . . . . 152

ourdissage . . . . . . . . . . 23

ouvraison. . . . . . . . . 17

ouvrés (fils de soie) . . . . . . I7

panne . . . . . . . . . . 6I

parapluie (soieries pour). . . . . . 151

passementiers (maitres). . . . . . I47

paysages en velours (Furnion) . . . I57

peau de soie. . . . . . . . . 50

peigne. . . . . . . . . . . . 30

pékin . . . . . . . . 78

peintes à la main (soieries) . . . . 145

peluche . . . . . . . . . 6r

perrotine. . . . . . . . . I37

perforage. . . . . . . . . 36

Ph. de Lasalle . . . . . . . . 84

pincettage. . . . . . . . . . . 32

piquage . . . . . . . . . 9I

piqûre. . . . . . . . . . . 3 I 
photographique (soie) planche (impression à la)

I 59

pliage

plissage

plumetis

poil (fil)

- (de velours).

- traînant (soierie à)

polissage .

pompadour (soierie)

pongée.

popeline

pou de soie.

prix de la soie (note') . . . . . 16

quadrillées (soieries).

quinze-seize (gros de Tours) . . . . 42

rabot (de velours)

rapport (du dessin)

ras... . . . . . 30

- de Saint-Cyr . . . . . . . 48

- de Saint-Maur. . . . . . . 48

réduction . . . . . . . . . . . 3 I

refaisage. . . . . . . . . 22

remettage . . . . . . . . 29

remisse . . . . . . . . . 29

reps. . . . . . . . . . . 53

ribandiers (maitres) . . . . . . . 147

rouleau (impression au). . . . . . I37

rubans découpés . . . . . . . I49

satin

— de Lyon . . . . . . . . 18

- à la reine. . . . . . . . . 50

- duchesse . . . . . . . . 50

- liberty. . . . . . . . . . 50

- merveilleux . . . . . . . 50

- météor. . . . . . . . 50

- romain. . . . . . . . . 50

- turc. . . . . . . . . 50

schappe (fil de). . . . . . . . I7

sergé . . . . . . . . 47

- composé . . . . . . . . 48

- croisé. . . . . . . . . 48

silésienne....... . . $f^{8}$, I5t

soie double teinte . . . . . . . . 22

soies solides á la cuite . . . . . . 22

soieries à plis tissés . . . . . . . 153

- circulaires: tournoises. . 55,153

- double étoffe. . . . . . 152

- double face... . . 55, 152 soicries sans envers.

teintes en pièce

- tissées en teints. . . . . . 18

- tramées verre . . . . . . ${ }_{152}$

surah . . . . . . . . . . 48

tabis . . . . . . . . 15

tableaux tissés en soie . . . . . 153

taffetaline. . . . . . . . 46

taffetas. . . . . . . . . . 4 I

- cannelé . . . . . . . . . $t^{2}$

— caméléon. . . . . . . . . 42

- glacé . . . . . . . . 42

- tramé cheveux. . . . . . 152

- tramé crin . . . . . . . I52

- tramé plume. . . . . . . . ${ }_{152}$

- tramé bois . . . . . . . I52

— tramé paille. . . . . . . I52

testament de Louis XVI (Maisiat) . . I57

thèses imprimées sur soie . . . . I59

tirelle . . . . . . . . . . . 3 I

tireur de lacs . . . . . . . . 92

tissotiers (maitres). . . . . . . . 147

toiles de soie. . . . . . . . . . 44

- (de velours). . . . . . . 57

trame . . . . . . . . ${ }_{17}$

tulle application . . . . . . . . 72

tulles de soie. . . . . . . . . . 70

twill. . . . . . . . $4^{8}$

velours à bouclés d'or. . . . . . . II

- à doubles pièces . . . . . 59

- á parterre ou jardinière . . Irg

- à plusieurs corps. . . . . . II6

- au fer. . . . . . . 57

- au sabre. . . . . . $52,14 t$

_- ciselé. . . . . . . I İ

- chinés . . . . . . . . . I35

- - de Richard . . . . I57

- deux hauteurs . . . . . 1 I 7

- du Nord. . . . . . . . 6т

- de Gênes . . . . . . . . II8

- de Paris. . . . . . . . . 59

— épinglé. . . . . . . bo

- frappé . . . . . . . . . I I

- frisé . . . . . . . . 60

- Grégoirc. . . . . . . . 157

-- mélangé d'or, teint en piéces. I20

- ottoman . . . . . . . 42

- poil schappe. . . . . . . 59

- Vauchelet. . . . . . . I57

voiles de soie... . . . . . . 64

unies, ou « en plein $\approx$ (soieries) . . . 39 



\section{TABLE DES ILLUSTRATIONS}

Tissage et broderie (d’après le Live de lingerie, 1584 ) . . . . . . . . . . . . . . I

Métier chinois (d'aprés Falcot). . . . . . . . . . . . . . . . . . 3

Atelier de tissage à bras de soieries, à Lyon . . . . . . . . . . . . . . . 8

Atelier de tissage mécanique de soieries . . . . . . . . . . . . . . . . . . 9

Ver à soie se nourrissant de feuilles de mủrier. . . . . . . . . . . . . . I 2

Cocon filé par le ver à soie. . . . . . . . . . . . . . . . . . . . . . Ij

Papillons de vers à soie . . . . . . . . . . . . . . . . . . . . . . . . . . . I3

La filature de la soie (d'après l'Encyclopédie) . . . . . . . . . . . . . . . I5

Flottes et bobines de soie. . . . . . . . . . . . . . . . . . . . I6

Moulinage de la soie . . . . . . . . . . . . . . . . . . . . . . . I7

Atelier de teinture de soieries (teinture en pièces) . . . . . . . . . . . . . 2 I

Dévidage mécanique de la soie . . . . . . . . . . . . . . . . . . . . . 23

Dévidage de la soie (d'aprés Paulet) . . . . . . . . . . . . . . . . . . 24

L'ourdissage (d'aprés l'Encyclopédie) . . . . . . . . . . . . . . . . . . 26

Détails du métier : battant, peigne, lisses. . . . . . . . . . . . . . . . 29

Navette ancienne, lancée à la main . . . . . . . . . . . . . . . . . . . 3 I

Navette moderne, employée pour le métier mécanique . . . . . . . . . . . 3 I

Atelier d'apprêt de soieries . . . . . . . . . . . . . . . . . . . . . 35

Rouleaux servant au gaufrage des soieries . . . . . . . . . . . . . . . 37

Schéma du taffetas . . . . . . . . . . . . . . . . . . . . . . . . fI

La calandre à moirer (d'après l'Encyclopédie) . . . . . . . . . . . . . . 43

Moire antique. . . . . . . . . . . . . . . . . . . . . . . . . 45

Moire française. . . . . . . . . . . . . . . . . . . . . 46

Schéma du sergé . . . . . . . . . . . . . . . . . . . . . . . . 47

Schèma du satin. . . . . . . . . . . . . . . . . . . . . . . . . 49

Reps. . . . . . . . . . . . . . . . . . . . . . . . . . . . 53

Cannelé . . . . . . . . . . . . . . . . . . . . . . . . . 54

Schèma du velours coupé (d’aprés l'Encyclopédie). . . . . . . . . . . . . 57

Schèma du velours frisé (d’après l'Encyclopédie) . . . . . . . . . . . . . 60

Schéma d’une gaze (d’après Peyot) . . . . . . . . . . . . . . . . . 65

Tulle alençon (grossi). . . . . . . . . . . . . . . . . . . . . . . 7 I

Tulle grec (grossi) . . . . . . . . . . . . . . . . . . . . . . . . II

Tulle dit à la chaine (grossiı. . . . . . . . . . . . . . . . . . . . . . I

Mètiers mécaniques de tulles . . . . . . . . . . . . . . . . . . . . 72

Tulle façonné (fabrication de Lron) . . . . . . . . . . . . . . . . . . T3

Tulle façonné (fabrication de Calais . . . . . . . . . . . . . . . . . . It

Soierie rayée par bandes de teintes différentes . . . . . . . . . . . . . . . 76

Soierie rayée par bandes d'armures et de teintes différentes . . . . . . . . . 77

Soierie barrée, ou bayadère, par armures différentes . . . . . . . . . . . 78

Soierie quadrillée . . . . . . . . . . . . . . . . . . . . . . . . 79

Portrait de Philippe de Lasalle. . . . . . . . . . . . . . . . . . . . 82

Dessin semé contresemplé . . . . . . . . . . . . . . . . . . . 84

Dessin semé sauté . . . . . . . . . . . . . . . . . . . . 85

Dessin ramagé ou à fond-plein. . . . . . . . . . . . . . . . . . . . 87 
Dessin pekine ou suivi. . . . . . . . . . . . . . . . . . . . . . . 88

Dessin à retour . . . . . . . . . . . . . . . . . 88

Exemple de mise en carte . . . . . . . . . . . . . . . . . . . . 89

Lisage des dessins daprés la mise en carte . . . . . . . . . . . . . . . . . 90

Exemple de soierie façonnée montrant le modelè obtenu par les différents points de trame

Nodéle dancien mitier ì la tire

9 I

92

Mécanique Jacquard . . . . . . . . . . . . . . . . . . . . . 94

Portrait de Jacquard, tissé en soie . . . . . . . . . . . . . . . . . . . 95

Métier à bras pour soieries façonnées . . . . . . . . . . . . . . . . . 97

Métier mécanique pour soieries façonnées. . . . . . . . . . . . . . . . 98

Damas d'ameublement, à decor satin, sur fond gros de Tours. . . . . . . . . 99

Brocatelle . . . . . . . . . . . . . . . . . 102

Lampas (modele du xvin ${ }^{\mathrm{e}}$ sicclel . . . . . . . . . . . . . . . . . . $10_{3}$

Schéma de la fabrication d'une soierie brochec (d'apres l'Encyclopedic) . . . . . I05

Soierie brochée, décor au faisan (composition de Philippe de Lasalle) . . . . . . 106

Grenadine brochée. . . . . . . . . . . . . . . . . . . . 107

Echarpe en mousseline brochee or . . . . . . . . . . . . . . . . . . 108

Solerie Pompadour modele du xvin sièclei. . . . . . . . . . . . . . . I Io

1)roguet. . . . . . . . . . . . . . . . . . . . . . . . II2

Crepe de Chine façonne et broche ur . . . . . . . . . . . . . . . . . II4

Velours avec bouclés d'or . . . . . . . . . . . . . . . . . . . I15

Velours de Genes . . . . . . . . . . . . . . . . . . . 116

Velours ciselé . . . . . . . . . . . . . . . . . . . . . . . . I I7

Telours miniature modele du xvin ${ }^{\circ}$ siecle) . . . . . . . . . . . . . . II8

Velours façonné, tramé or, teint en piece . . . . . . . . . . . . . . . . . . II9

Brocart or, argent et soic . . . . . . . . . . . . . . . . . . . I 23

Soierie façonnée moirée . . . . . . . . . . . . . . . . . . . . . . 126

Soierie brodec (composition de Philippe de Lasalle) . . . . . . . . . . . . I29

Robe de l'époque Louis XV en taffetas chiné. . . . . . . . . . . . . . . I34

Atelier dimpression sur solerie . . . . . . . . . . . . . . . . . . . I36

Soierie impriméc par enlevage. . . . . . . . . . . . . . . . . . . . . . . . . 137

Planche d'impression gravè en relief sur bois .

Ouvrier imprimeur, appliquant sur l'étoffe la planche qu'il vient de garnir de couleur sur le chássis plaçe à sa droite . . . . . . . . . . . . . . . . I I I

Soierie dameublement, imprimée sur chaine . . . . . . . . . . . . . . . I4

Ruban fond satin avec décor de velours au sabre . . . . . . . . . . . . . I 15

Pinceau pulvérisateur de couleurs d’aérographe . . . . . . . . . . . . . If6

Nétier de rubans . . . . . . . . . . . . . . . . . . . . I48

Rubans anciens . . . . . . . . . . . . . . . . . . . . . . . . I50

"Le Jardinier ", soierie puur siege : cumposition de Philippe de Lasalle . . . . . I53

Portrait tissé de Napoléon Irr, par Gantillon. . . . . . . . . . . . . . . I55

Tissu dit de Cologne . . . . . . . . . . . . . . . . . . . . . . I56

Velours Grégoire (Musée de Marseille). . . . . . . . . . . . . . . . . I58

Impression genre estampe sur satin (époque du Premier Empire) . . . . . . . I60 


\section{TABLE DES MATIĖRES}

Avant-propos. . . . . . . . . . . . . . . . . . . . . . . . . . I

Quelques mots d"histoire : Vieilles soies, jeunes chiffons. . . . . . . . . . . I

Comment sobtient la soie . . . . . . . . . . . . . . . . . . . . . I2

Teinture de la soie. . . . . . . . . . . . . . . . . . . . . . . . . . . . . . 19

Fabrication, dévidage, ourdissage . . . . . . . . . . . . . . . . . . 23

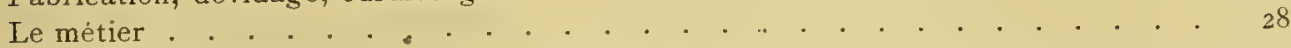

Les industries de finissage . . . . . . . . . . . . . . . . . . . . . 34

Comment dénommer et classer les soieries . . . . . . . . . . . . . . . . $3^{8}$

Le taffetas. . . . . . . . . . . . . . . . . . . . . . . . . . 4I

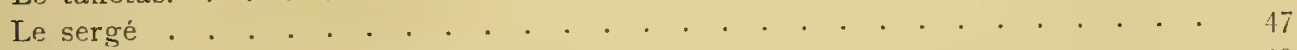

Le satin. . . . . . . . . . . . . . . . . . . . . . . . . . . . 419

Reps, cannelé, armures diverses . . . . . . . . . . . . . . . . . . . 53

Le velours . . . . . . . . . . . . . . . . . . . . . . . . . . . . . 56

La gaze. . . . . . . . . . . . . . . . . . . . . . . . . . . . . . . . 62

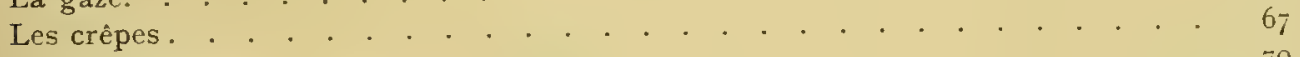

Les tulles de soie . . . . . . . . . . . . . . . . . . . . . . . . . . . . . . . . . 70

Les soieries à décor: Rayures et carreaux . . . . . . . . . . . . . . . 75

Soieries façonnées ou à dessins tissés . . . . . . . . . . . . . . . . . . 80

Damas, brocatelle, lampas . . . . . . . . . . . . . . . . . . . . . . . 99

Soicries brochées . . . . . . . . . . . . . . . . . . . . . . 105

Soieries façonnées par la chaîne: pompadour, droguet et double chaîne . . . . . IIo

Gazes, crépes et velours façonnés . . . . . . . . . . . . . . . . . . Irt

Brocarts . . . . . . . . . . . . . . . . . . . . . . . . . . I2I

Broderies sur soie . . . . . . . . . . . . . . . . . . . . . . . 127

Soies peintes, soieries imprimées . . . . . . . . . . . . . . . . . . . . . I32

Rubans de soie. . . . . . . . . . . . . . . . . . . . . . . . I IT

Spécialités et curiosités du tissage de la soie et de son décor . . . . . . . . . I5I

Table analytique des matières. . . . . . . . . . . . . . . . . . . . I63

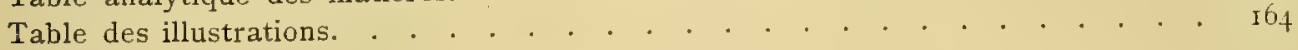









SMITHSONIAN INSTITUTION LIBRARIES

3908800337796 ?

chm NK8906.A592

Grammaire des arts de la soie. 